\begin{tabular}{l|l|l|l|l} 
2. To: (Receiving Organization) & 3. From: (Originating Organization)
\end{tabular}

Air \& Water Services

5. Proj./Prog./Dept./Div.:

WCS/ES

8. Originator Remarks:

\section{Fro appermal}

11. Receiver Remarks:
Air \& Water Services

6. Cog. Engr.:

B. P. Gleckler

Page 1 of

1. EDT 601694

4. Related EDT No.:

601536

7. Purchase Order No.:

$\mathrm{N} / \mathrm{A}$

9. Equip./Component No.:

$\mathrm{N} / \mathrm{A}$

10. System/Bldg./Facility:

$N / A$

12. Major Assm. Dwg. No.:

$N / A$

13. Permit/Permit Application No.: $\mathrm{N} / \mathrm{A}$

14. Required Response Date:

$05 / 16 / 96$

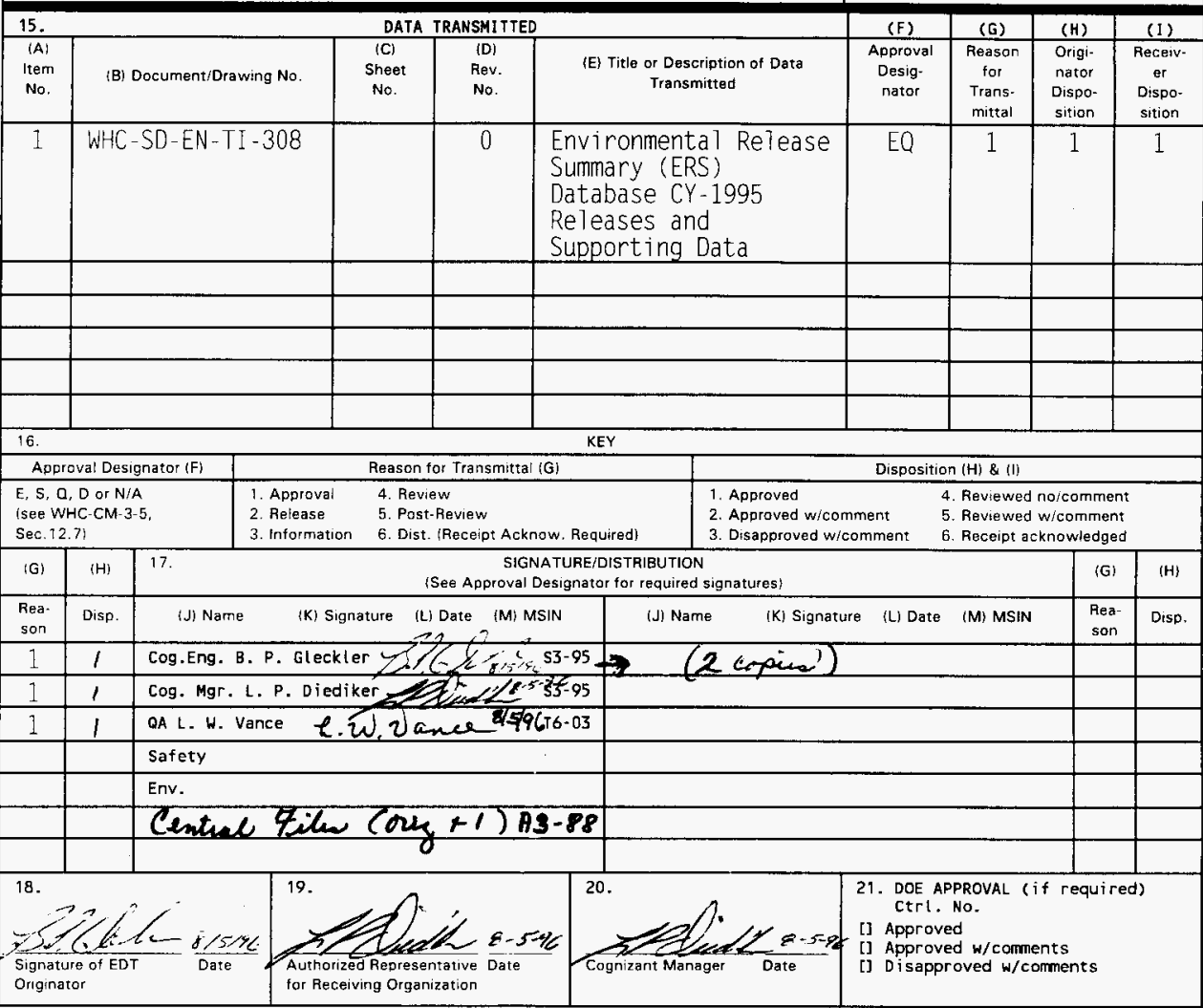




\section{Environmental Release Summary (ERS) Database CY 1995 Releases and Supporting Data}

B. P. Gleckler

Westinghouse Hanford Co.. Richland. WA 99352

U.S. Department of Energy Contract DE-AC06-87RL10930

$\begin{array}{lll}\text { EDT/ECN: } & 601694 & \text { UC: } 630 \\ \text { Org Code: } & 01871 & \text { Charge Code: R43U1 } \\ \text { B\&R Code: } & \text { EW3120100 } & \text { Tota? Pages: } 3 / 6 \mathrm{cw}_{8-6-\% 6}\end{array}$

Key Words: ERS. Environmental Release Summary. stack releases. point source emissions, liquid effluents.

Abstract: This document is a hard copy of the CY 1995 airborne and 7 iquid effluent data contained in the Environmental Release Summary (ERS) computer database.

TRADEMARK DISCLAIMER. Reference herein to any specific comercial product, process, or service by trade name, trademark, manufacturer, or otherwise, does not necessarily constitute or imply its endorsement, recommendation, or favoring by the United States Government or any agency thereof or its contractors or subcontractors.

Printed in the United States of America. To obtain copies of this document, contact: WHC/BCS Document Control Services, P.O. Box 1970, Mailstop H6-08, Richland WA 99352, Phone (509) 372-2420; Fax (509) $376-4989$

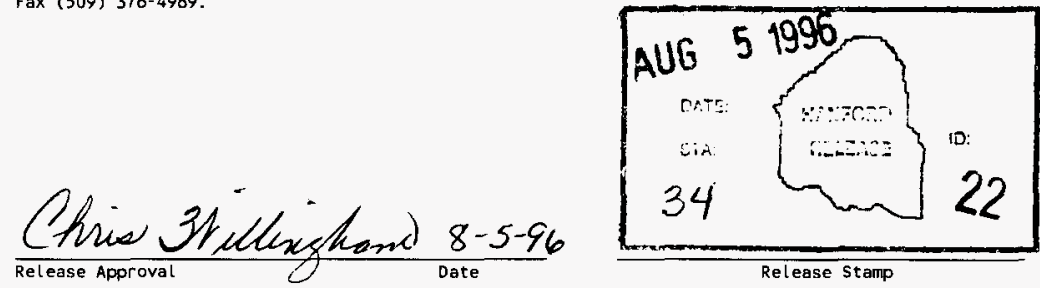

\section{Approved for Public Release}




\title{
Environmental Release Summary (ERS) Database CY 1995 Releases and Supporting Data
}

\author{
B. P. Gleckler \\ Westinghouse Hanford Company \\ Date Published \\ May 1996
}

Prepared for the U.S. Department of Energy

Assistant Secretary for Environmental Management

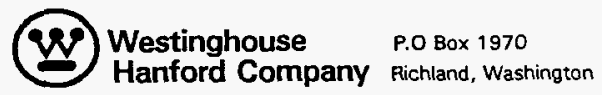

Management and Operations Contractor for the

U.S. Department of Energy under Contract DE-ACO6-87RL10930

Approved for public release; distribution is unlimited 


\section{ENVIRONMENTAL RELEASE SUMMARY (ERS) DATABASE CY-1995 RELEASES AND SUPPORTING DATA \\ CONTENTS}

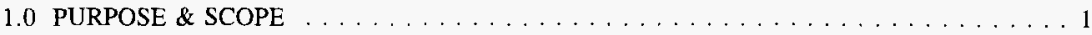

2.0 AIR EFFLUENT RELEASES \& DATA $\ldots \ldots \ldots \ldots \ldots \ldots \ldots \ldots \ldots$

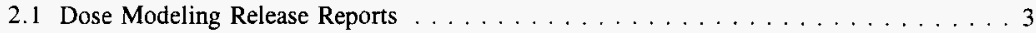

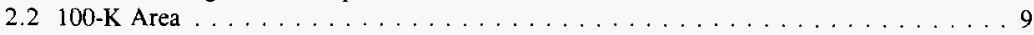

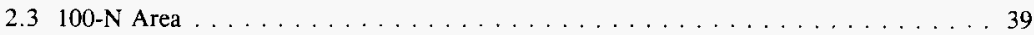

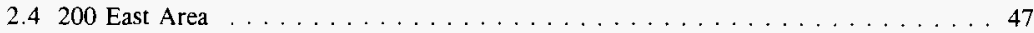

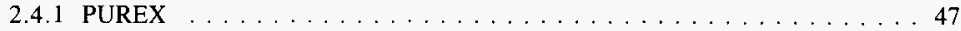

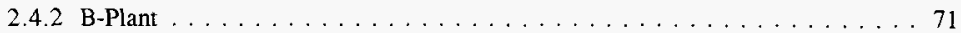

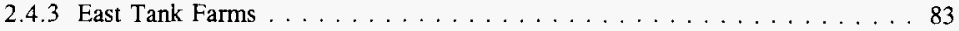

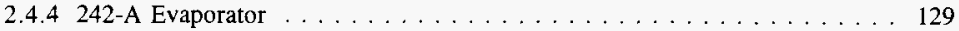

2.4.5 Effluent Treatment Facility (ETF) . . . . . . . . . . . . . . 137

2.5200 West Area . . . . . . . . . . . . . . . . . . . . . . . . 141

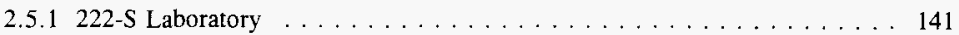

2.5 .2 T-Plant . . . . . . . . . . . . . . . . . . . . . . . 147

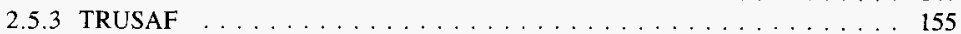

2.5 .4 West Tank Farms . . . . . . . . . . . . . . . . . . . 161

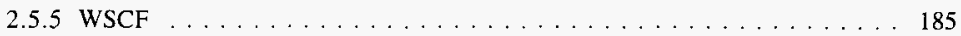

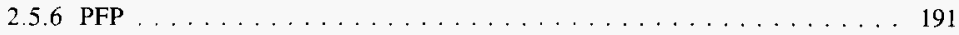

$2.5 .7200-$ W BHI Facilities . . . . . . . . . . . . . . . . . . 207

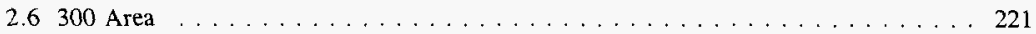

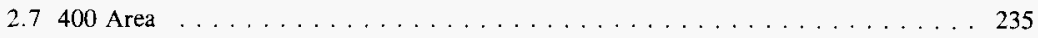

3.0 LIQUID EFFLUENT RELEASES \& DATA . . . . . . . . . . . . . . . . . . 249

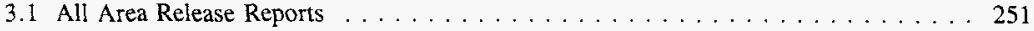

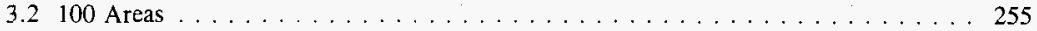

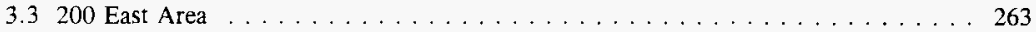

3.4200 West Area . . . . . . . . . . . . . . . . . . 277

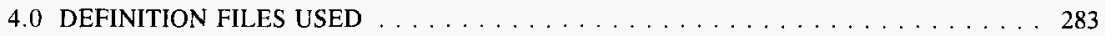

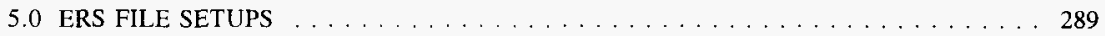

5.1 Audit Report for Air Effluent Files . . . . . . . . . . . . . . . . . . . . 291

5.2 Audit Report for Liquid Effluent Files . . . . . . . . . . . . . . . . . . . 303

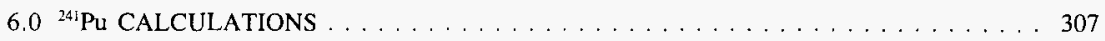


This page intentionally left blank. 


\subsection{PURPOSE AND SCOPE}

The Environmental Release Summary (ERS) database is a "living" database. The database is continually modified to fulfill the current needs of Air \& Water Service. Changes made, to accurately calculate current releases, effect how past releases are calculated. This document serves as a "snap-shot" of the database and software for the CY-1995 data and releases.

This document contains all of the relevant data for calculating radioactive airborne and liquid effluent releases from Westinghouse Hanford Company (WHC) and Bechtel Hanford Incorporated (BHI) facilities, for CY-1995. The ERS database is the official repository for all WHC and BHI effluent sample data. This document serves as the repository for the CY-1995 ERS release reports and the settings used to generate those reports.

As part of the Tri-Party Agreement, WHC Air \& Water Services has committed to the Washington State Department of Ecology to maintain a hard copy of the ERS database, since Ecology does not have direct access to the computer database. This document also serves as the hard copy, that WHC has committed to have available.

\subsection{AIR EFFLUENT RELEASES \& DATA}

This section contains all of the effluent air release reports and the sample collection and analysis supporting data. Sub-section 2.1 contains the air emission releases calculated for each major operating area. The remaining sub-sections include the sample collection and analysis supporting data (trend reports) and radionuclide airborne releases (release reports) for individual emission points or source terms. No release or trend reports were included for those emission points or source terms which did not operate in CY-1995. 
WHC-SD-EN-TI-308 Rev.0

This page intentionally left blank. 
WHC-SD-EN-TI-308 Rev.0

2.1 Dose Modeling Release Reports 
Radionuclides Discharged to the Atmosphere from 100 Areas ** 01/01/95 through $12 / 31 / 95 * \star *$

Total system Flow: $3 \cdot 1 \mathrm{E}+12$ Liters $=1 \cdot 1 \mathrm{E}+11$ Cubic Ft

Radionu
${ }^{60} \mathrm{Co}$
${ }^{90} \mathrm{Sr}$
${ }^{106} \mathrm{Ru}$
${ }^{125} \mathrm{Sb}$
${ }^{134} \mathrm{Ce}$
${ }^{137} \mathrm{Ce}$
${ }^{154} \mathrm{Eu}$
${ }^{155} \mathrm{Eu}$
${ }^{238} \mathrm{Pu}$
$239 / 240 \mathrm{Pu}$
${ }^{241} \mathrm{Pu}$
$241 \mathrm{Am}$

Halfife
5.27 Years
29.12 Years
368 Days
2.8 Years
2.1 Years
30 Years
8.8 Years
4.96 Years
87.8 Years
24,131 Years*
14.4 Years
432 Years

Release, $\mathrm{Ci}$

9. 2E-06

6. $1 E-05$

1. 1E-05

2. $8 \mathrm{E}-06$

1. $3 \mathrm{E}-06$

2. $5 \mathrm{E}-04$

8. 3E-06

1. $5 \mathrm{E}-06$

2. 3E-06

1. $5 \mathrm{E}-05$

2. $1 E-04$

5. $7 E-06$

* The halflife shown for Total Uranium is the halflife of ${ }^{238} \mathrm{U}$. The halflife shown for mixed ${ }^{239 / 240} \mathrm{Pu}$ is the halflife of ${ }^{239} \mathrm{Pu}$.

** Values for one or more files projected backward to cover report period.

*** Values for one or more files projected forward to cover report period. 
WHC-SD-EN-TI-308 Rev.0

$08 / 01 / 96$

ENVIRONMENTAL RELEASE SUMMARY REPORT

Radionuclides Discharged to the Atmosphere from 200-East Area

$01 / 01 / 95$ through $12 / 31 / 95$

Total System Flow: $4.9 \mathrm{E}+12$ Liters $=1.7 \mathrm{E}+11$ Cubic Ft

$\begin{array}{ccc}\text { Radionuclide } & \text { Halflife } & \text { Release, Ci } \\ { }^{90} \mathrm{Sr} & 29.12 \text { Years } & 6.8 \mathrm{E}-05 \\ { }^{103} \mathrm{Ru} & 39 \text { Days } & \mathrm{ND} \\ { }^{106} \mathrm{Ru} & 368 \text { Days } & 6.7 \mathrm{E}-06 \\ { }^{113} \mathrm{Sn} & 115 \text { Days } & 8.0 \mathrm{E}-07 \\ { }^{125} \mathrm{Sb} & 2.8 \text { Years } & 9.1 \mathrm{E}-06 \\ { }^{129} \mathrm{I} & 1.57 \mathrm{E}+7 \text { Years } & 8.9 \mathrm{E}-03 \\ { }^{131} \mathrm{I} & 8.04 \text { Days } & \mathrm{ND} \\ { }^{134} \mathrm{Cs} & 2.1 \text { Years } & 2.4 \mathrm{E}-08 \\ { }^{137} \mathrm{Cs} & 30 \text { Years } & 3.8 \mathrm{E}-04 \\ { }^{152} \mathrm{Eu} & 13.3 \text { Years } & 3.7 \mathrm{E}-07 \\ { }^{154} \mathrm{Eu} & 8.8 \text { Years } & 4.6 \mathrm{E}-07 \\ { }^{155} \mathrm{Eu} & 4.96 \text { Years } & 2.2 \mathrm{E}-07 \\ { }^{238} \mathrm{Pu} & 87.8 \text { Years } & 6.9 \mathrm{E}-07 \\ 239 / 240 \mathrm{Pu} & 24,131 \text { Years* } & 7.9 \mathrm{E}-06 \\ { }^{241} \mathrm{Pu} & 14.4 \text { Years } & 1.2 \mathrm{E}-04 \\ { }^{24} \mathrm{Am} & 432 \text { Years } & 7.6 \mathrm{E}-05\end{array}$

${ }^{90} \mathrm{Sr}$ values include Total $\mathrm{B}$ measurements from emission sources which are not analyzed for Sr.

$239 / 240 \mathrm{Pu}$ value includes Total $\alpha$ measurements from emission sources which are not analyzed for Pu.

* The halflife shown for Total Uranium is the halfIife of ${ }^{238} \mathrm{U}$. The halflife shown for mixed $239 / 24{ }^{\circ} \mathrm{Pu}$ is the halflife of ${ }^{239} \mathrm{Pu}$.

*** Values for one or more files projected forward to cover report period. 


\section{WHC-SD-EN-TI-308 Rev.0}

$08 / 01 / 96$

ENVIRONMENTAL RELEASE SUMMARY REPORT

Radionuclides Discharged to the Atmosphere from 200 -West Area ** 01/01/95 through $12 / 31 / 95 * \star *$

Total system Flow: $8.4 \mathrm{E}+12$ Liters $=3.0 \mathrm{E}+11$ Cubic $\mathrm{Ft}$

$\begin{array}{ccc}\text { Radionuclide } & \text { Halflife } & \text { Release, Ci } \\ { }^{90} \mathrm{Sr} & 29.12 \text { Years } & 1.0 \mathrm{E}-04 \\ { }^{106} \mathrm{Ru} & 368 \text { Days } & 4.0 \mathrm{E}-09 \\ { }^{113} \mathrm{Sn} & 115 \text { Days } & 2.7 \mathrm{E}-09 \\ { }^{125} \mathrm{Sb} & 2.8 \text { Years } & 2.4 \mathrm{E}-08 \\ { }^{134} \mathrm{Cs} & 2.1 \text { Years } & 2.0 \mathrm{E}-08 \\ { }^{137} \mathrm{Cs} & 30 \text { Years } & 1.9 \mathrm{E}-05 \\ { }^{152} \mathrm{Eu} & 13.3 \text { Years } & 5.6 \mathrm{E}-08 \\ { }^{154} \mathrm{Eu} & 8.8 \text { Years } & 1.4 \mathrm{E}-07 \\ { }^{155} \mathrm{Eu} & 4.96 \text { Years } & 1.2 \mathrm{E}-07 \\ { }^{239} \mathrm{Pu} & 87.8 \text { Years } & 2.6 \mathrm{E}-06 \\ 239 / 24{ }^{\circ} \mathrm{Pu} & 24.131 \text { Years*} & 1.0 \mathrm{E}-04 \\ { }^{241} \mathrm{Pu} & 14.4 \text { Years } & 2.0 \mathrm{E}-04 \\ { }^{241} \mathrm{Am} & 432 \text { Years } & 1.7 \mathrm{E}-05\end{array}$

${ }^{90} \mathrm{Sr}$ values include Total $\beta$ measurements from emission sources which are not analyzed for Sr.

${ }^{239 / 240} \mathrm{Pu}$ value includes Total $\alpha$ measurements from emission sources which are not analyzed for $\mathrm{Pu}$.

* The halflife shown for Total Uranium is the halfIife of ${ }^{238} \mathrm{U}$. The halflife shown for mixed $239 / 240 \mathrm{Pu}$ is the halflife of ${ }^{239} \mathrm{Pu}$.

** Values for one or more files projected backward to cover report period.

*** Values for one or more files projected forward to cover report period. 


\section{WHC-SD-EN-TI-308 Rev.0}

WHC ENVIRONMENTAL RELEASE SUMMARY

Radionuclides Discharged to the Atmosphere from the 300 Area

** 01/01/95 through $12 / 31 / 95 \star \star \star$

Total system Flow: $3.4 \mathrm{E}+11$ Liters $=1.2 \mathrm{E}+10$ Cubic Ft

Radionuclide

${ }^{90} \mathrm{Sr}$

${ }^{131} \mathrm{I}$

${ }^{137} \mathrm{Cs}$

Total U

$239 / 240 \mathrm{Pu}$

${ }^{90} \mathrm{Sr}$ values include Total $B$ measurements from emission sources which are not analyzed for sr.

Total $U$ value includes Total a measurements from emission sources which are not analyzed for $U$.

$239 / 240$ pu value includes Total a measurements from emission sources which are not analyzed for $P u$.

* The halflife shown for Total Uranium is the halflife of ${ }^{238} \mathrm{U}$. The halflife shown for mixed $239 / 240$ pu is the halflife of ${ }^{239} \mathrm{Pu}$.

* Values for one or more files projected backward to cover report period.

*** Values for one or more files projected forward to cover report period. 
WHC ENVIROMMENTAL RELEASE SUMMARY

Radionuclides Discharged to the Atmosphere from the 400 Area * * $01 / 01 / 95$ through $12 / 31 / 95$

Total System Flow: $1.1 E+12$ Liters $=3.9 E+10$ Cubic Ft

$$
\text { Radionuclide }
$$

${ }^{3} \mathrm{H}$

$131 \mathrm{I}$

$13^{7} \mathrm{Cs}$

$239 / 24 \mathrm{CPu}_{\mathrm{Pu}}$

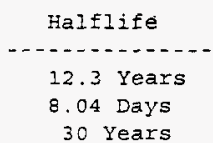

24, 131 Years*
Release, Ci

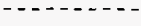

2. 5E-02 ND

5. 9 E- 05

1. $7 E-06$

${ }^{137} \mathrm{Cs}$ values include Total B measurements from emission sources which are not analyzed for Cs.

239:24c Pu value includes Total $\alpha$ measurements from emission sources which are not analyzed for Pu.

* The halfiife shown for Total Uranium is the halflife of $23 \mathrm{U}$. The halflife shown for mixed $239 / 24$ Pu is the halflife of

* Values for one or more files projected backward to cover report period.

*** Values for one or more files projected forward to cover report period. 
WHC-SD-EN-TI-308 Rev.0

$2.2100-\mathrm{K}$ Area 
WHC ENVIRONMENTAL RELEASE SUMMARY

105-KE BASIN ROOF VENTS (Y245-Y24B)

$01 / 01 / 95$ through $12 / 31 / 95$

Total System Flow: $5.3 E+11$ Liters $=1.9 E+10$ Cubic Ft

Radionuclide
${ }^{60} \mathrm{Co}$
${ }^{90} \mathrm{Sr}$
${ }^{106} \mathrm{Ru}$
${ }^{125} \mathrm{Sb}$
${ }^{134} \mathrm{Cs}$
${ }^{137} \mathrm{Cg}$
${ }^{154} \mathrm{Eu}$
${ }^{155} \mathrm{Eu}$
${ }^{238} \mathrm{Pu}$
$239 / 240 \mathrm{Pu}$
${ }^{241} \mathrm{Pu}$
$241 \mathrm{Am}$
Total $\alpha$
Total $\beta$

\begin{tabular}{c} 
Halflife \\
\hline 5.27 Years \\
29.12 Years \\
368 Days \\
2.8 Years \\
2.1 Years \\
30 Years \\
8.8 Years \\
4.96 Years \\
87.8 Years \\
24,131 Years* \\
14.4 Years \\
432 Years \\
n/a \\
n/a
\end{tabular}

Release, $\mathrm{Ci}$.

1. $9 \mathrm{E}-06$

5. 9z-05

1. IE-05

2. 5E-06

4. $6 \mathrm{E}-07$

2. $4 \mathrm{E}-04$

5. 8E-06

1. $2 \mathrm{E}-06$

2. $3 E-06$

1. $5 \mathrm{E}-05$

2. $1 \mathrm{E}-04$

5. $6 \mathrm{E}-06$

2. $7 E-05$

3. $\mathrm{OE}-04$

* The halflife shown for Total Uranium is the halflife of ${ }^{238} \mathrm{U}$. The halflife shown for mixed ${ }^{239 / 240} \mathrm{Pu}$ is the halflife of ${ }^{239} \mathrm{Pu}$. 


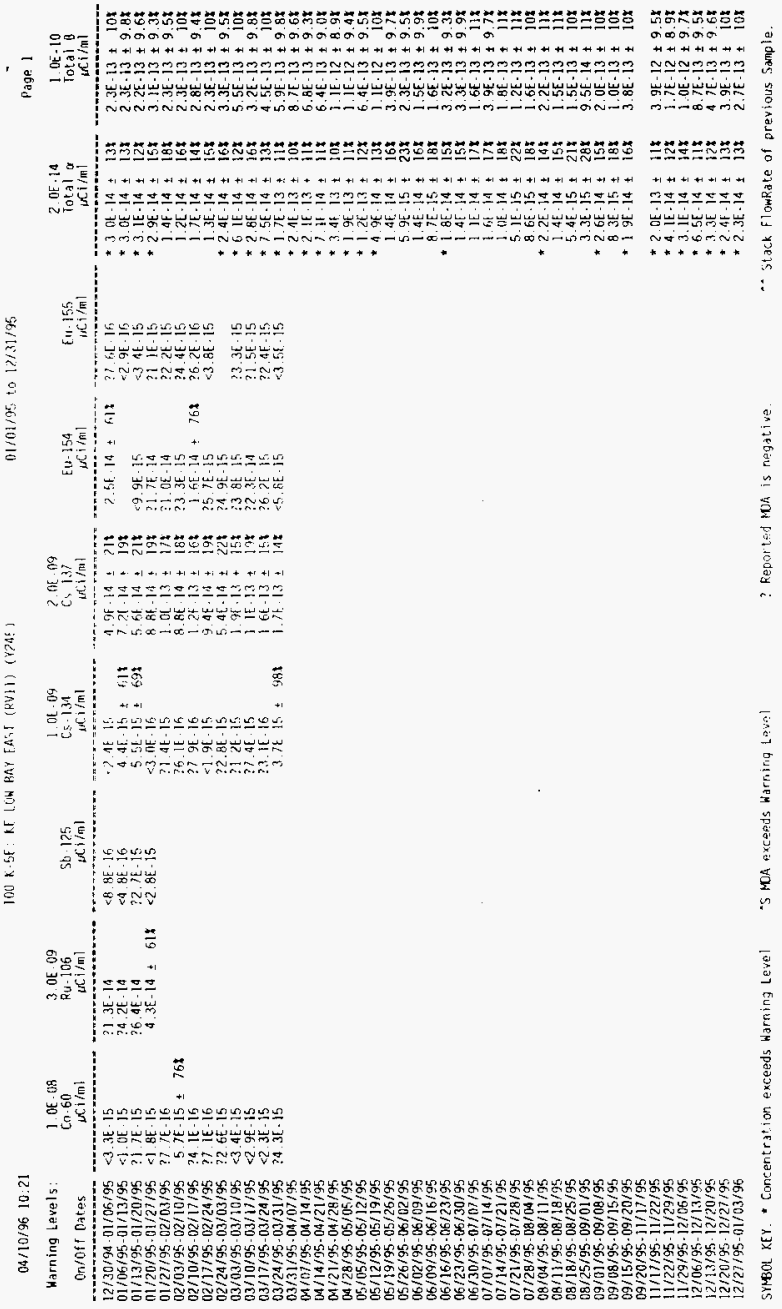


04/10/96 10:21

100-k.5E: KE LOW PAYY LAST IFYHD) (Y2A5)

$011 / 01 / 75$ to $12731 / 95$

Fage 2

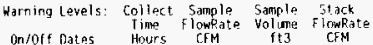

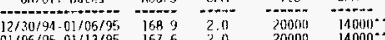

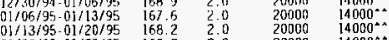

$\begin{array}{lllll}01 / 20 / 95-01 / 27 / 95 & 168.0 & 2.0 & 20000 & 14000^{\circ} \\ 01 / 27 / 95.02 / 03 / 95 & 167.7 & 2.0 & 20000 & 4000^{\circ}\end{array}$

$\begin{array}{lllll}022 / 03 / 95.02 / 10 / 95 & 167.8 & 2.0 & 20000 & 14000^{\wedge}\end{array}$

$\begin{array}{llllll}02 / 10 / 95.02 / 17 / 95 & 168.2 & 2.0 & 20000 & 14000^{\star} \\ 022 / 1 / 95.02 / 24 / 95 & 169.2 & 2.0 & 20000 & 14000^{*}\end{array}$

$\begin{array}{lllll}02 / 24 / 95-03 / 03 / 95 & 168.7 & 2.0 & 20000 & 14000^{\circ}\end{array}$

$\begin{array}{lllll}03 / 03 / 95-03 / 10 / 95 & 166.8 & 2.0 & 20000 & 14000^{\circ} \\ 03 / 10 / 95-03 / 1 / 95 & 157.7 & 2.0 & 30060 & 14000^{\circ}\end{array}$

$\begin{array}{llllll}0.3 / 17 / 95.03 / 24 / 95 & 167.8 & 2.0 & 20000 & 14000^{\circ} \\ 0.320 / 95.03 / 3 / 95 & 158.3 & 2.0 & 20000 & 14000^{\circ}\end{array}$

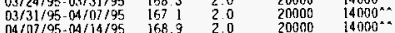

$\begin{array}{lllll}04 / 07 / 95.04 / 14 / 95 & 168.9 & 2.0 & 20000 & 140010 \times \\ 04 / 14 / 95-04 / 21 / 95 & 167.8 & 2.0 & 200000 & 141100^{\circ}\end{array}$

$\begin{array}{lllll}04 / 4 / 95-04 / 21 / 95 & 167.8 & 2.0 & 200000 & 144160 \% \\ 04 / 21 / 95-0 / 23 / 23 / 95 & 167.9 & 2.0 & 20000 & 14000^{\circ}\end{array}$

$\begin{array}{llllll}4 / 28 / 95-(15 / 05 / 95 & 168.2 & 2.0 & 20000 & 14000^{*} \\ 5 / 05 / 95-05 / 12 / 95 & 168.1 & 2.0 & 20000 & 14000^{*}\end{array}$

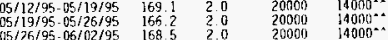

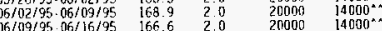

$\begin{array}{llllll}06 / 16 / 95-06 / 23 / 95 & 168.0 & 2.0 & 20000 & 14000^{\wedge}\end{array}$

$06 / 30 / 95-17 / 07 / 95$ 168? $20 \quad 20000014000^{\circ}$

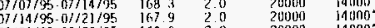




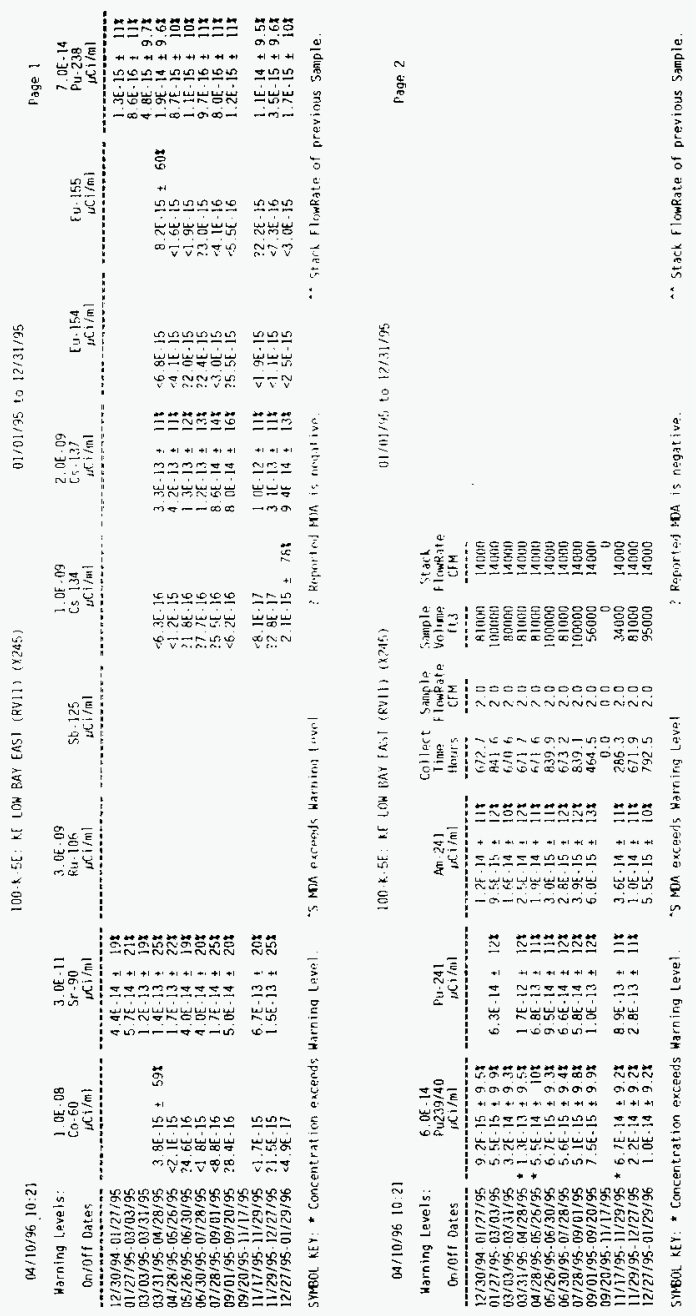




\section{WHC-SD-EN-TI-308 Rev.0}

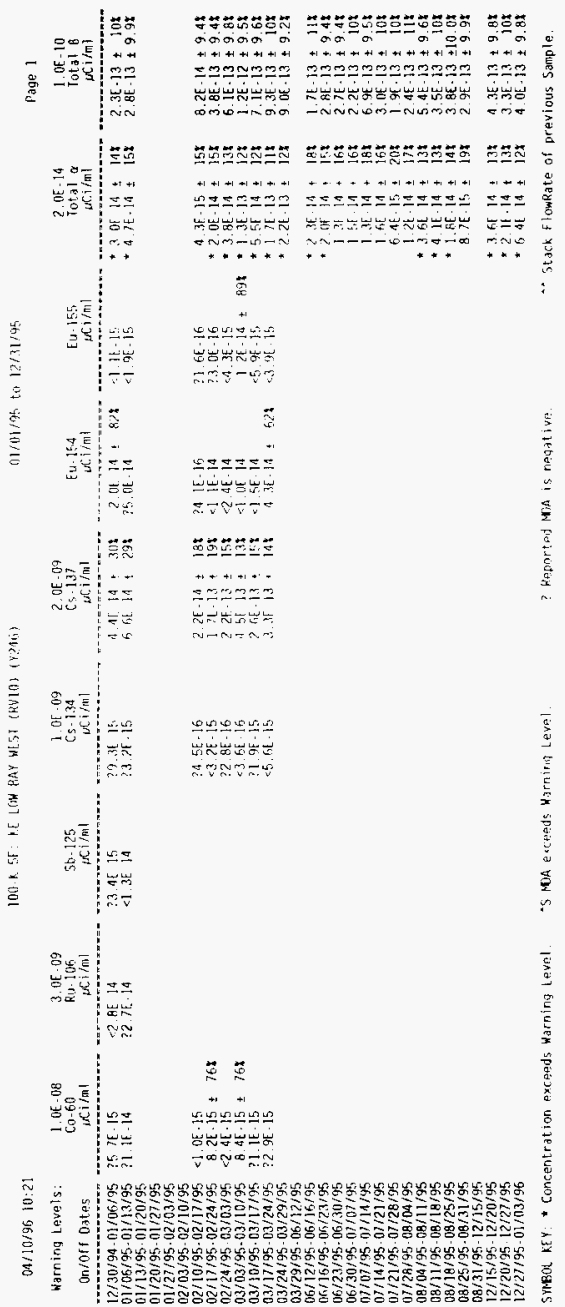



我信

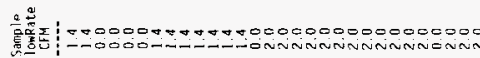

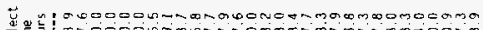

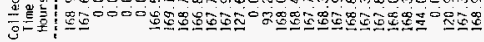
군

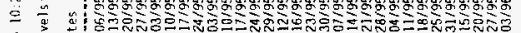
4 I

至 \& E

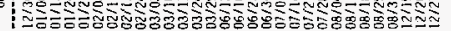




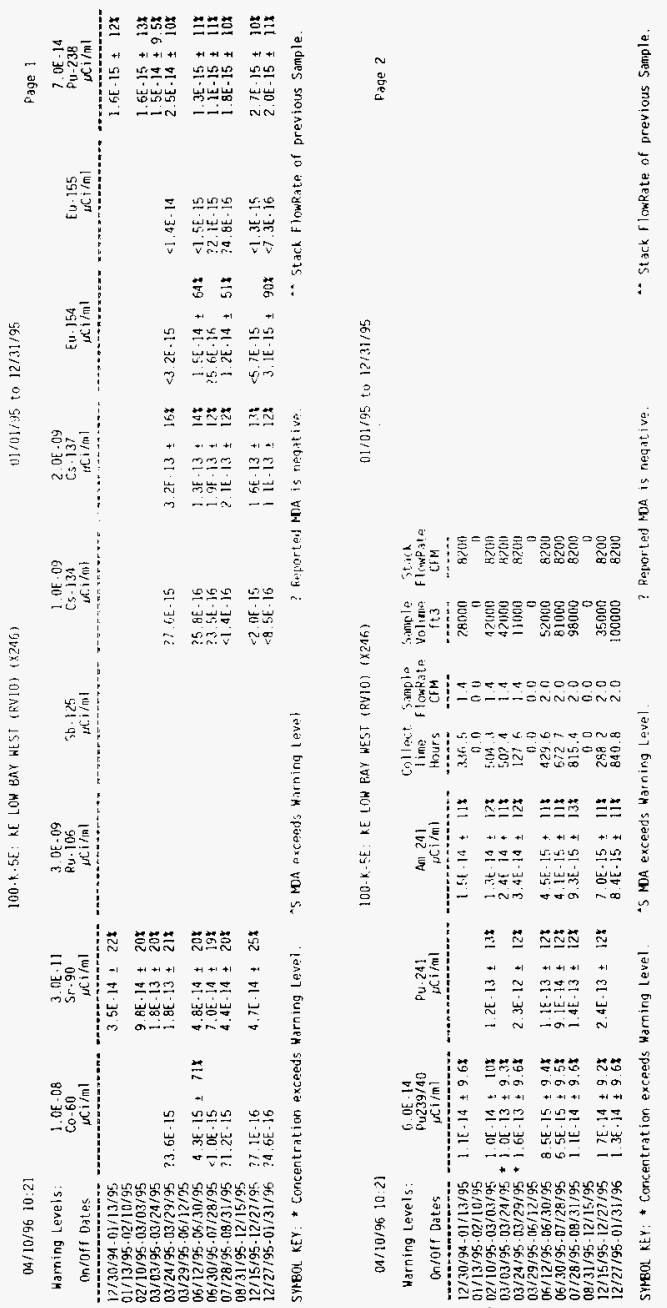




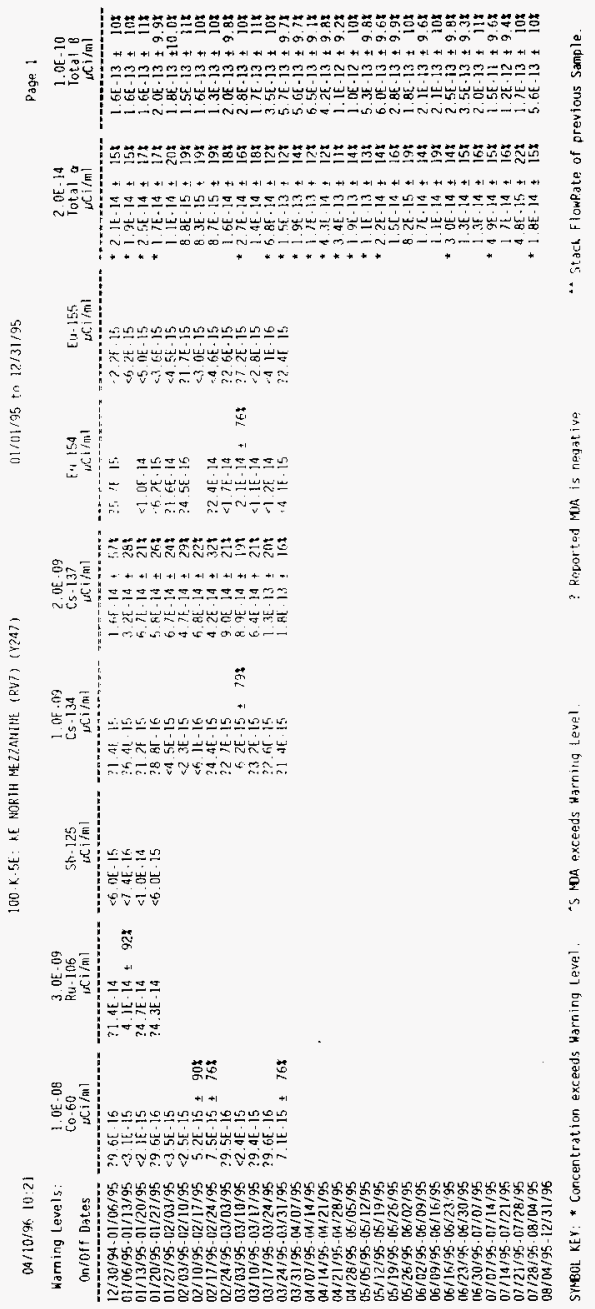


04/10/96 10:21

Harnirg leveis: Collect Sample sample slack

Den/Off Dates Hours CFM C $\mathrm{Ct3}$ CFM

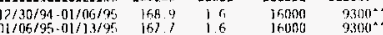

$\begin{array}{llllll}01 / 13 / 95.01 / 20 / 95 & 169.1 & 1.6 & 16000 & 9300^{\circ} \\ 01 / 20 / 95.01 / 22195 & 169.0 & 1.6 & 1600 & 9300 \%\end{array}$

$\begin{array}{lllll}01 / 20 / 95.01 / 27 / 95 & 169.0 & 1.6 & 16000 & 9300 * \\ 01 / 27 / 95.02 / 03 / 95 & 167.6 & 1.6 & 16000 & 93000\end{array}$

$\begin{array}{llllll}02 / 03 / 95.02 / 10 / 95 & 168.0 & 1.5 & 16000 & 9300^{\circ}\end{array}$

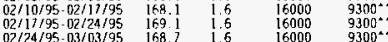

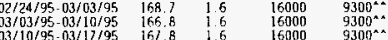

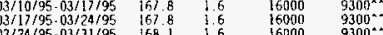

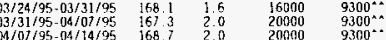

$\begin{array}{llllll}03 / 0 / 95-04 / 4 / 95 & 168.7 & 2.0 & 20000 & 9300^{\circ} \\ 104 / 14 / 95.04 / 21 / 95 & 167.8 & ? .0 & 20600 & 9300^{\circ} .\end{array}$

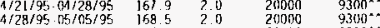

$05 / 05 / 95.015 / 12 / 95 \quad 167$ ह $2.0 \quad 20000 \quad 93000^{2-}$

$\begin{array}{lllll}05 / 19 / 95.05 / 26 / 95 & 169.1 & 2.0 & 20000 & 93700 \\ 0.1 & 2.0 & 20000 & 9300 \%\end{array}$

$\begin{array}{llll} & 26295.06 / 09 / 95 & 168.6 & 2.0 \\ 168 & 2.0\end{array}$

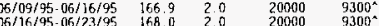

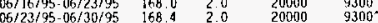

66/30/95-0//07/95 $167.7 \quad 2.0 \quad 20090 \quad 9300^{\circ}$ 


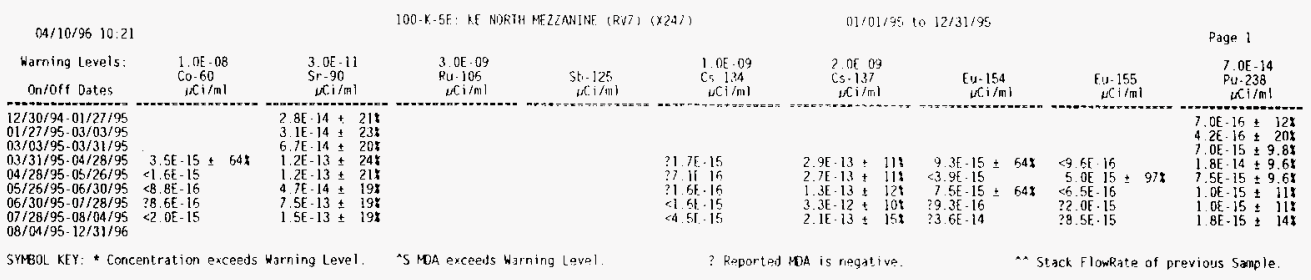
$94 / 10 / 96 \quad 10.21$

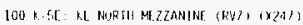
$01 / 011 \% 1 \%+1001 / 31 / 9$
Page 2

\begin{tabular}{|c|c|c|c|c|c|c|c|}
\hline $\begin{array}{l}\text { Warning Levels: } \\
\text { On/off Oates }\end{array}$ & $\begin{array}{l}6.0 \mathrm{E}-14 \\
\mathrm{Puz} 39 \mathrm{~g} / \mathrm{ho} \\
\mu \mathrm{C} \mathrm{i} / \mathrm{mI}\end{array}$ & 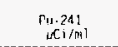 & Nin-241 & $\begin{array}{l}\text { Collext } \\
\text { Time } \\
\text { Howrs }\end{array}$ & $\begin{array}{l}\text { Fanple } \\
\text { Flowkits } \\
\text { CFM }\end{array}$ & $\begin{array}{l}\text { Sample } \\
\text { voluplo } \\
\text { ft.? }\end{array}$ & 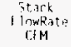 \\
\hline 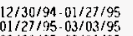 & & $4.0 E \cdot 14 \pm 16 t$ & $\begin{array}{ll}15 \pm 119 \\
15 \pm 128\end{array}$ & & & $\begin{array}{l}8,5000 \\
81000\end{array}$ & $\begin{array}{l}9300 \\
9300\end{array}$ \\
\hline $103 / 95.03 / 31 / 95$ & $4.6 \mathrm{E}-14 \pm 94 \mathrm{I}$ & & $-14 \pm 12 x$ & 670.5 & 1.6 & 1000 & 9300 \\
\hline $\begin{array}{l}95.04 / 28 / 95+ \\
95.05 / 26 / 95\end{array}$ & $+1.2 E \cdot 13+958$ & $\begin{array}{l}1.7 \mathrm{E} \cdot 12 \\
5.8 \mathrm{E} \cdot 1.3\end{array}$ & $\begin{array}{l}2.36-14+124 \\
1.85 .14 \div 198\end{array}$ & 671 & $\frac{2}{3}$ & singen & 9300 \\
\hline 95.064 & $6.8 \mathrm{E} 15$ t & $9.5 \mathrm{~F}=12 \mathrm{t}$ & tof of 15,113 & Bat ? & $?$ & (1)1⿴囗i) & , \\
\hline $06 / 30 / 95.07 / 2$ & $6.16 \cdot 15$ & $6.9 \mathrm{E} \cdot 14 \pm 124$ & I. of $14=$ iit & & ? & 1416000 & 9300 \\
\hline $\begin{array}{l}07 / 28 / 95 \cdot 09 / 04 / 95 \\
08 / 04 / 95 \cdot 12 / 31 / 96\end{array}$ & 1.0E-14 & $1.4 E \cdot 13 \pm 13 t$ & $5.8 E-15 \pm 148$ & $\begin{aligned} 175.1 \\
0.0\end{aligned}$ & 200 & 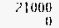 & $\begin{array}{r}330 j \\
0\end{array}$ \\
\hline
\end{tabular}

SYGOL kEY * Concentration exceeds Warning Level. is Ma excoefls Harniny Level. 


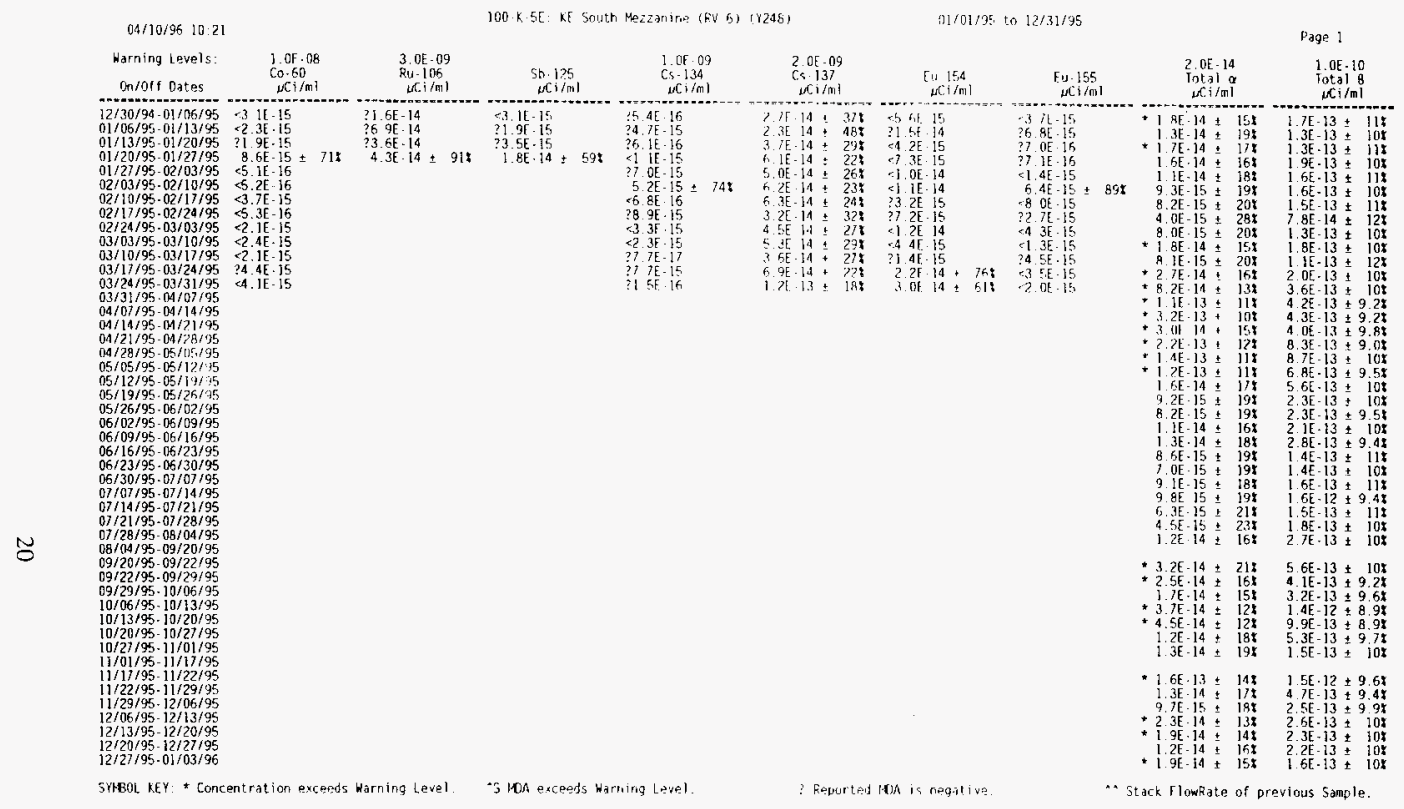




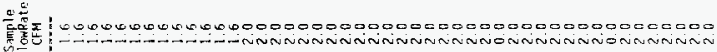

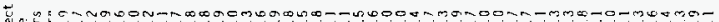
至|

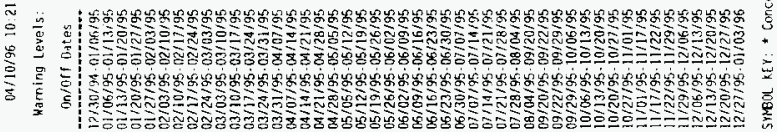




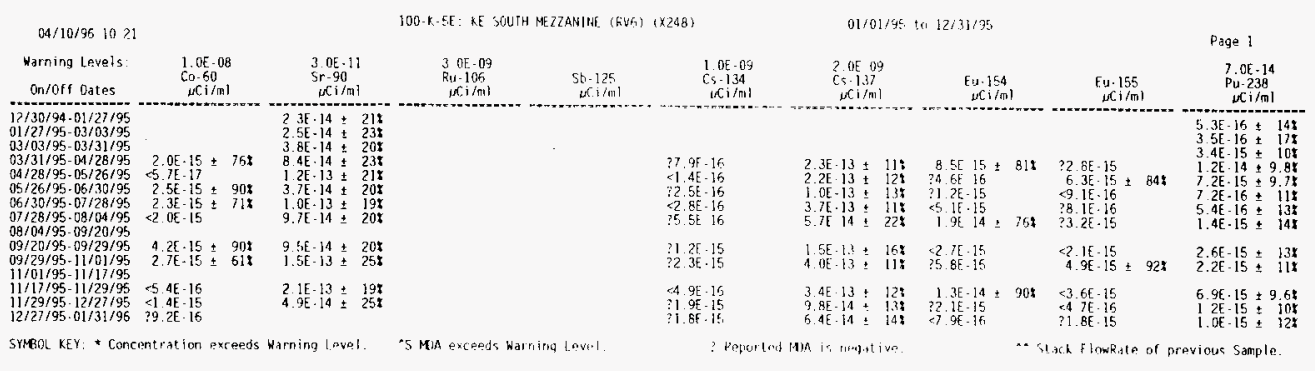

$04 / 10 / 95 \quad 10: 21$

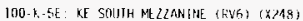

$01 / 01 / 95$ to $12 / 31 / 05$

\begin{tabular}{|c|c|c|c|c|c|c|c|}
\hline $\begin{array}{l}\text { Harning levels: } \\
\text { Onroff bates }\end{array}$ & 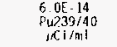 & $\begin{array}{l}\mathrm{Pu} \cdot 241 \\
\mathrm{~m} \mathrm{C}, \mathrm{i} / \mathrm{m}\}\end{array}$ & $\begin{array}{l}\text { An } 241 \\
\left|S_{0} ; / m\right|\end{array}$ & $\begin{array}{l}\text { Collect } \\
\text { Tigne } \\
\text { lheirs }\end{array}$ & 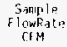 & $\begin{array}{c}\text { sample } \\
\text { yolinas } \\
\text { it.? }\end{array}$ & $\begin{array}{l}\text { sitatk } \\
\text { Fiawiate } \\
\text { IIM }\end{array}$ \\
\hline $\begin{array}{l}13 / 30 / 94-0.121 / 95 \\
01 / 27 / 95.03 / 03 / 95\end{array}$ & $\begin{array}{l}3.7 \mathrm{E}-15 \pm 9.98 \\
2.4 \mathrm{E} \cdot 15 \pm 11 \mathrm{t}\end{array}$ & $2.8 E-14 \pm 15 t$ & $\begin{array}{l}5 \text { at } 15 \div 1 \\
3 \text { of } 15 \pm 134\end{array}$ & $\begin{array}{l}\text { (ii)? } \\
\text { Bail. }\end{array}$ & 16 & 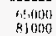 & $\begin{array}{l}16,0901 \\
16000\end{array}$ \\
\hline $\begin{array}{l}03 / 03 / 95.03 / 31 / 95 \\
03 / 31 / 95.01 / 28 / 95\end{array}$ & $\begin{array}{l}2.2 E \cdot 14 \pm 9.48 \\
\times \quad 3\end{array}$ & & $15 \pm 128$ & 6705 & 10 & $640061]$ & 16000 \\
\hline $04 / 28 / 95.05 / 26 / 95$ & $4.96 .14 \pm 9.4 t$ & 118 & $16 \mathrm{E} \cdot \mathrm{l}^{2} \pm 10 \mathrm{t}$ & $\begin{array}{l}51.6 \\
671.5\end{array}$ & 2.0 & 8. & $\begin{array}{l}166000 \\
36000\end{array}$ \\
\hline $05 / 26 / 95-06 / 30 / 95$ & $45 \mathrm{E} \cdot 15 \pm 95$ & 5.0E $14 \pm 128$ & 3 AE.-15 \pm 118 & 840.5 & $\frac{2}{2}$ & 100000 & 16,000 \\
\hline $\begin{array}{l}06 / 30 / 95-01 / 28 / 95 \\
07 / 28 / 95.08 / 04 / 95\end{array}$ & $\begin{array}{l}3.0 \mathrm{E}-15 \pm 9.8 \mathrm{x} \\
7.6 \mathrm{E}-15 \pm 10 \mathrm{y}\end{array}$ & $\begin{array}{l}3.7 \mathrm{E} \cdot 14 \pm 134 \\
9.7 \mathrm{E}-14 \pm 14 \mathrm{x}\end{array}$ & 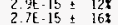 & 672.5 & & 81000 & 16000 \\
\hline $08 / 04 / 95-09 / 20 / 95$ & & & & & & 0 & 16000 \\
\hline $\begin{array}{l}09 / 20 / 95.09 / 29 / 95 \\
09 / 29 / 95.11 / 01 / 95\end{array}$ & $\begin{array}{l}1.5 \mathrm{E} \cdot 14 \\
1 . \mathrm{AE} \cdot 1 \mathrm{th}\end{array}$ & $\begin{array}{l}2.1 \mathrm{E}-13 \pm 135 \\
1.8 \mathrm{E} \cdot 13 \pm 12 \mathrm{~K}\end{array}$ & $\begin{array}{l}15 \pm 13 t \\
E .15 \pm 12 t\end{array}$ & & & & $\begin{array}{l}25000 \\
25000\end{array}$ \\
\hline $\begin{array}{l}11 / 01 / 95.11 / 11 / 95 \\
11 / 17 / 95.11 / 29 / 95 \\
11 / 29 / 95-12 / 27 / 95 \\
12 / 21 / 95.01 / 31 / 96\end{array}$ & $\begin{array}{l}4 \cdot 3 E \cdot 14 \pm 9 \cdot 14 \\
3.4 E \cdot 15 \pm 9 \cdot 38 \\
6 \cdot 9 E \cdot 15 \pm 9 \cdot 88\end{array}$ & $\begin{array}{l}5.8 E-13 \pm 11: \\
1.1 E-13 \pm 124\end{array}$ & $\begin{array}{l}\text { 2.4E. } 14 \pm 11 \% \\
3.8 \mathrm{E} .15 \pm \\
4.0 \mathrm{E} .15 \pm \\
11 \%\end{array}$ & $\begin{array}{l}286.4 \\
672.2 \\
341.0\end{array}$ & $\begin{array}{l}2.0 \\
2.0\end{array}$ & $\begin{array}{r}0 \\
34000 \\
81000 \\
100000\end{array}$ & $\begin{array}{r}25000 \\
2500000 \\
25000\end{array}$ \\
\hline
\end{tabular}

srubol kEY: * Concentration exceeds Warning level. $\because$, Mod exceeds Harning Level 
WHC-SD-EN-TI-308 Rev.0

WHC ENVIRONMENTAL RELEASE SUMMARY

$04 / 10 / 96$

105-KW BASIN ROOF VENTS (Y234-Y236)

$01 / 01 / 95$ through $12 / 31 / 95$

Total System Flow: $5.0 E+11$ Liters $=1.8 \mathrm{E}+10$ Cubic Ft

Radionuclide
${ }^{60} \mathrm{Co}$
${ }^{90} \mathrm{Sr}$
${ }^{106} \mathrm{Ru}$
${ }^{125} \mathrm{Sb}$
${ }^{134} \mathrm{Cs}$
${ }^{137} \mathrm{Cs}$
${ }^{154} \mathrm{Eu}$
${ }^{255} \mathrm{Eu}$
${ }^{238} \mathrm{Pu}$
$239 / 2{ }^{40} \mathrm{Pu}$
${ }^{241} \mathrm{Pu}$
$241 \mathrm{Am}$
Total a
Total $B$

Halflife
5.27 Years
29.12 Years
368 Days
2.8 Years
2.1 Years
30 Years
8.8 Years
4.96 Years
87.8 Years
24,131 Years*
14.4 Years
432 Years
n/a
$n / a$

Release, Ci .

5. 7E-07

1. $0 E-06$

ND

ND

8. $6 \mathrm{E}-07$

1. 3E-05

1. $9 \mathrm{E}-06$

7. 8E-09

1. $2 \mathrm{E}-08$

7. $0 \mathrm{E}-08$

1. $4 \mathrm{E}-06$

5. $9 \mathrm{E}-08$

1. $7 \mathrm{E}-06$

2. 2E-05

* The nalflife shown for Total Uranium is the halflife of ${ }^{238} \mathrm{U}$. The halflife shown for mixed ${ }^{239 / 240} \mathrm{Pu}$ is the halflife of ${ }^{239} \mathrm{Pu}$. 


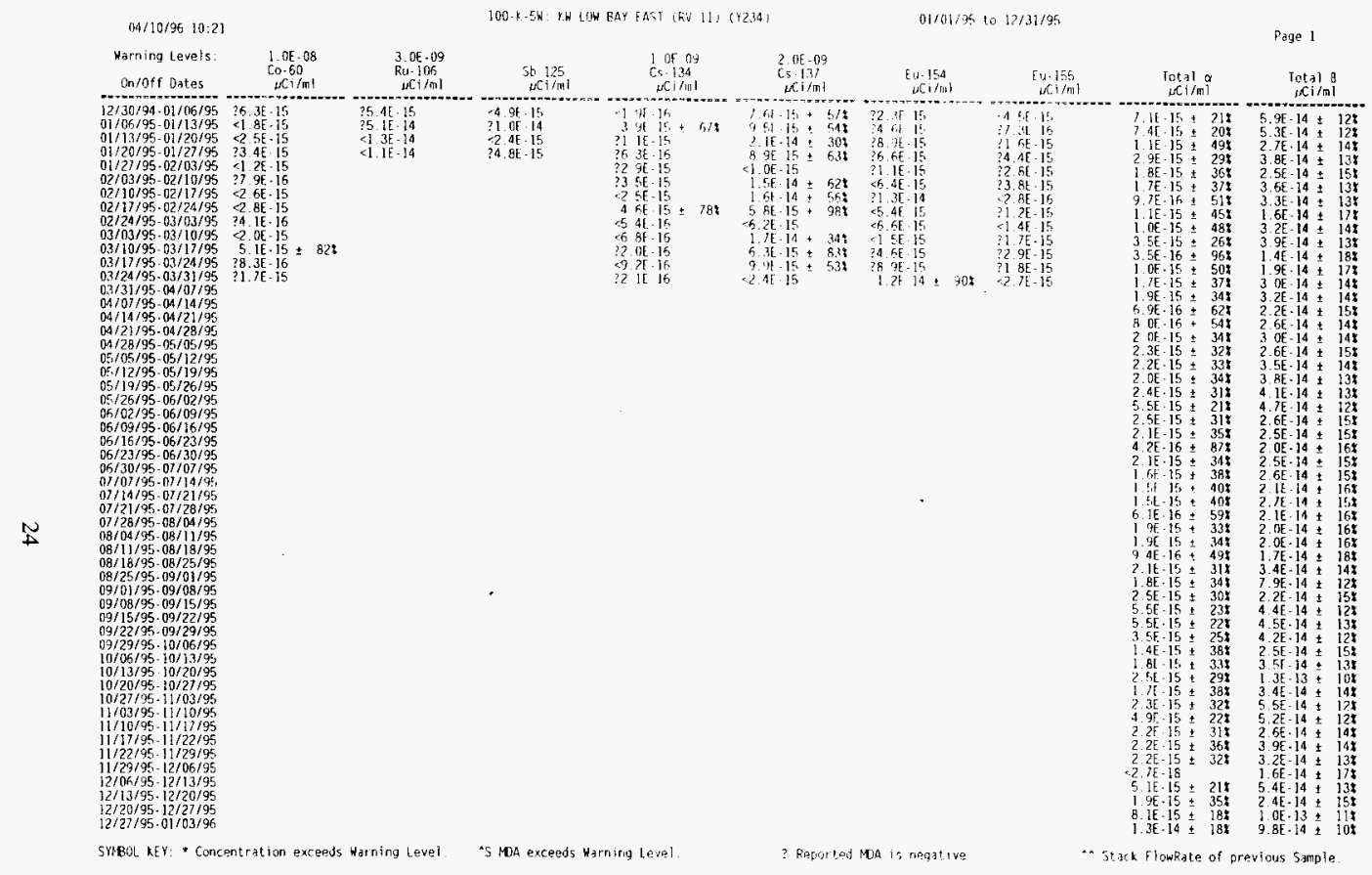




$a$
0

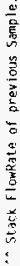

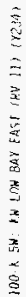

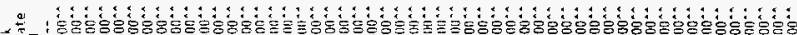

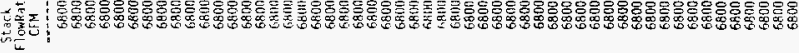

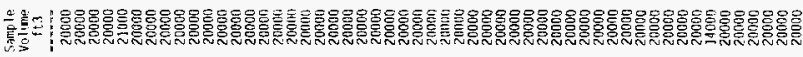

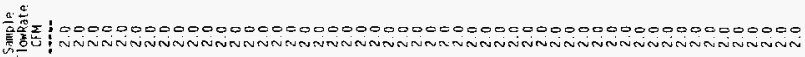

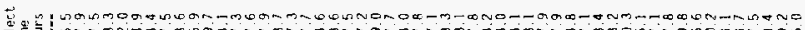

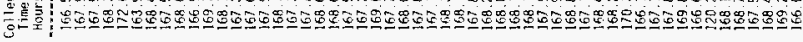

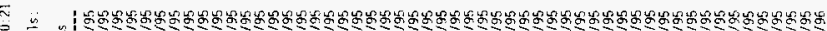
\% \%

军

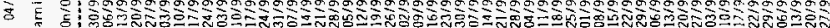

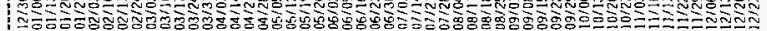




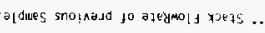

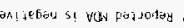

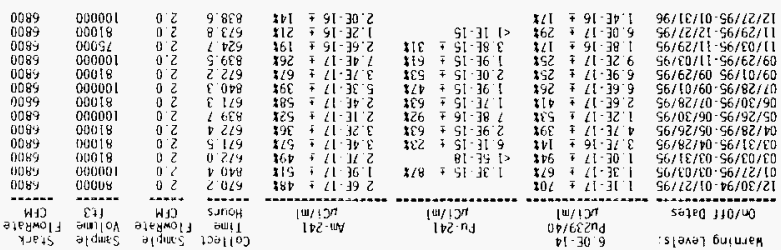

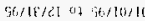

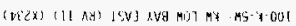

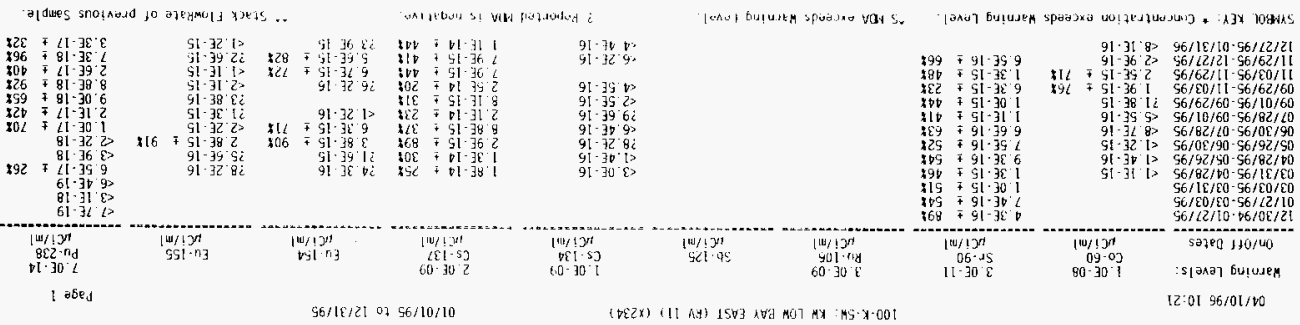




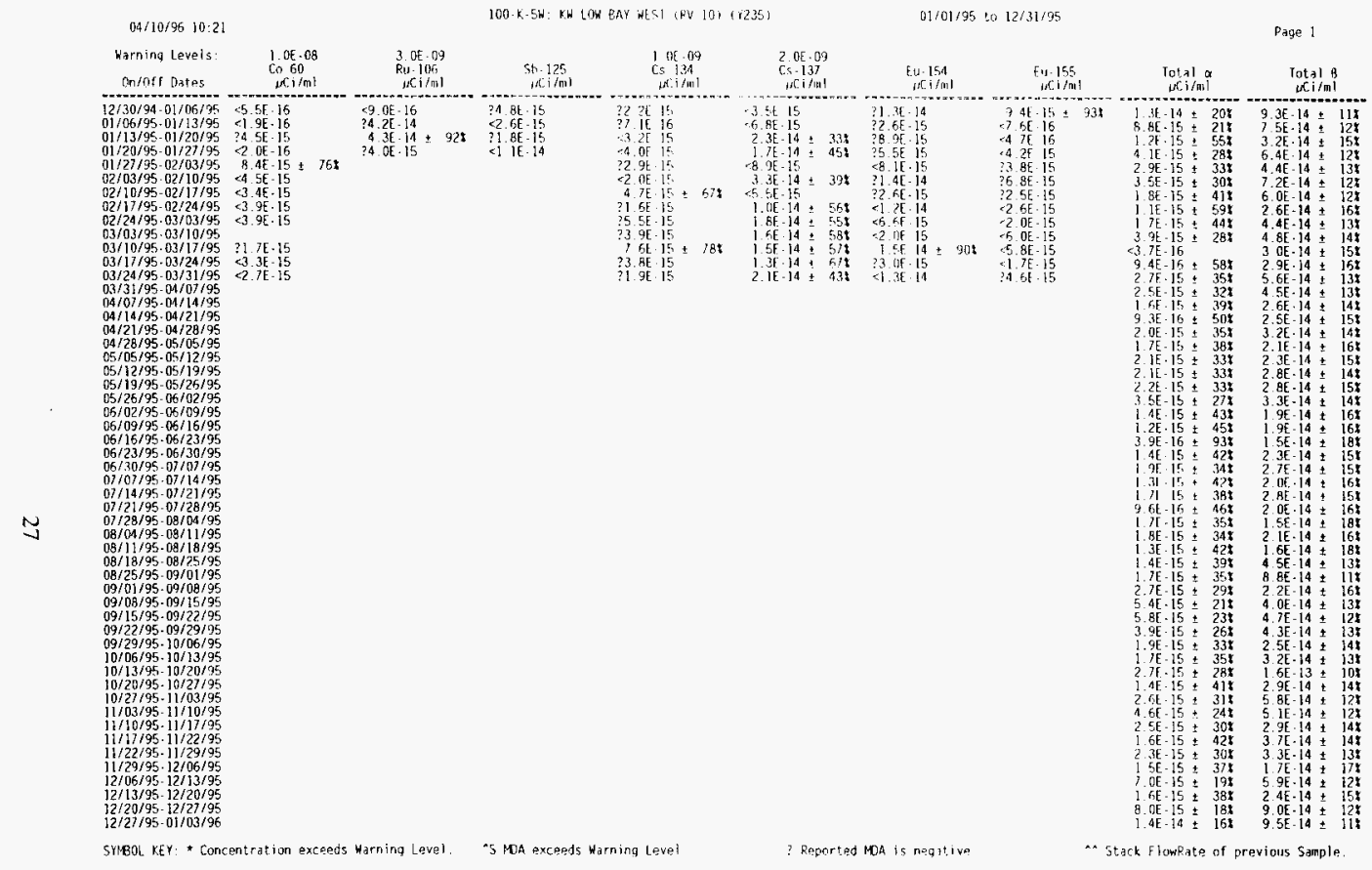


$04 / 10 / 96 \quad 10: 21$

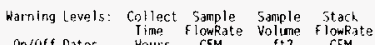

\begin{tabular}{|c|c|c|c|c|}
\hline On/off lates & $\begin{array}{l}\text { Time } \\
\text { Hours }\end{array}$ & $\begin{array}{l}\text { flowfate } \\
\text { CFM }\end{array}$ & $\begin{array}{l}\text { Volune } \\
\mathrm{ft} 3\end{array}$ & $\begin{array}{l}\text { flowfate } \\
\text { CFM }\end{array}$ \\
\hline $12 / 30 / 94-01 / 46 / 95$ & 165.5 & $b_{5}$ & 15000 & $4,3011^{\circ}$ \\
\hline $\begin{array}{l}01 / 06 / 95-01 / 13 / 95 \\
01 / 13 / 95-01 / 20 / 95\end{array}$ & $\begin{array}{l}167.9 \\
167.5\end{array}$ & 5 & $\begin{array}{l}55000 \\
5000\end{array}$ & $\begin{array}{l}4300^{\circ} \\
4300^{-1}\end{array}$ \\
\hline $01 / 20 / 95,01 / 27 / 95$ & 168.3 & & 15000 & $4300^{\circ}$ \\
\hline $\begin{array}{l}01 / 27 / 95-02 / 03 / 95 \\
02 / 03 / 95-02 / 10 / 95\end{array}$ & 172.0 & & 15000 & $4300^{\circ}$ \\
\hline $02 / 10 / 95.02 / 1) / 95$ & $\begin{array}{l}15.9 \\
158.4\end{array}$ & & 15000 & 年300 \\
\hline $02 / 17 / 95-02 / 24 / 95$ & 157.4 & & 15000 & $4300^{x}$ \\
\hline $02 / 24 / 95-03 / 03 / 95$ & 168.3 & & 55000 & $4300^{\circ}$ \\
\hline $03 / 03 / 95-03 / 10 / 95$ & 165.9 & & 15000 & $4300^{\circ}-$ \\
\hline $03 / 1 / 95-03 / 17 / 95$ & 1697 & 5 & 15000 & $4300^{\circ}$ \\
\hline $\begin{array}{l}03 / 1795.03 / 24 / 95 \\
03 / 24 / 95.03 / 31 / 95\end{array}$ & $\begin{array}{l}158.1 \\
167.3\end{array}$ & .5 & $\begin{array}{l}15000 \\
5000\end{array}$ & $\begin{array}{l}4300^{\circ} \\
4300^{\circ}\end{array}$ \\
\hline $03 / 31 / 95-04 / 107 / 95$ & 167.6 & 20 & 20000 & $4300^{2}$ \\
\hline $104 / 01 / 95.14 / 14 / 95$ & 167.9 & & 20000 & $43000^{\circ}$ \\
\hline $\begin{array}{l}01 / 14 / 95-01 / 21 / 95 \\
04 / 21 / 95-04 / 28 / 95\end{array}$ & 168.7 & $2^{2}$ & 20000 & $\begin{array}{l}4,300^{\circ}-4 \\
4300^{\circ}\end{array}$ \\
\hline $04 / 28 / 95.05 / 05 / 95$ & 1673 & 20 & $\begin{array}{l}200000 \\
20000\end{array}$ & 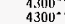 \\
\hline $05 / 05 / 95-05 / 12 / 95$ & 1686 & 20 & 20000 & $4300^{\circ}$ \\
\hline $05 / 12 / 95-05 / 19 / 95$ & 168.6 & 2 & 20000 & $4300^{\circ}$ \\
\hline $\begin{array}{l}05 / 19 / 95-05 / 26 / 95 \\
05 / 26 / 35.05 / 02 / 195\end{array}$ & 167.4 & $\begin{array}{l}2 \\
2\end{array}$ & 20000 & $\begin{array}{l}4300^{\circ} \\
4300^{\circ}\end{array}$ \\
\hline $06 / 02 / 95-06 / 09 / 95$ & 1689 & 20 & $\begin{array}{l}200000 \\
20000\end{array}$ & $\begin{array}{l}4300^{\circ} \\
4300^{\circ}\end{array}$ \\
\hline $06 / 09 / 95-06 / 16 / 95$ & 367.7 & 2.0 & 20090 & $4300^{-1}$ \\
\hline $06 / 16 / 95-05 / 23 / 95$ & 158.0 & 20 & 20000 & $4300^{\circ}$ \\
\hline $\begin{array}{l}606 / 233 / 95.06 / 30 / 95 \\
06 / 30 / 95-07 / 07 / 95\end{array}$ & 167.8 & 2.0 & 20000 & $\begin{array}{l}4300^{\circ} \\
4,300^{4}\end{array}$ \\
\hline & $\operatorname{lin} 4$ & 30 & 2tithlat & $\begin{array}{l}4.7000^{2} \\
1.100^{2}\end{array}$ \\
\hline 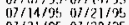 & J & & $20,010)$ & 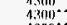 \\
\hline $0721 / 95-n 7 / 28 / 35$ & 1578 & 2.0 & 20000 & $4300^{x}$ \\
\hline 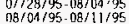 & $\begin{array}{l}168.2 \\
158.0\end{array}$ & $\begin{array}{l}2.0 \\
2.0\end{array}$ & $\begin{array}{l}20000 \\
20090\end{array}$ & $\begin{array}{l}4300^{*} \times \\
4300^{*}\end{array}$ \\
\hline $5-08118 / 95$ & 168 & 2.0 & 20000 & 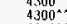 \\
\hline$-08 / 25 / 95$ & & & 20000 & 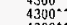 \\
\hline & & & 20000 & (4.300 \\
\hline 09 & & & 20000 & $4300^{\circ}$ \\
\hline 099 & 168 & & 20000 & $4300^{\circ}$ \\
\hline $09 / 15 /$ & & & 20000 & $4300^{\circ}$ \\
\hline $2 / 95.09 / 29$ & & & & $4300^{*}:$ \\
\hline $5.10 / 06 / 95$ & 168 & & 20000 & $4300^{*} \times$ \\
\hline 15.10? & & & 20000 & $43 \pi n 0^{*}$ \\
\hline $55 \cdot 10 \%$ & & & 20000 & \\
\hline $10 / 27 / 95.11 / 03 / 95$ & & & 20000 & 4300 \\
\hline $3 / 95-11 / 10 / 95$ & & & 0000 & $4.300^{2 \mathrm{x}}$ \\
\hline 5 & & & 20000 & $4300^{-1}$ \\
\hline 15.11 & $i_{2}+2 \cdot a$ & & 14000 & $4.300^{-2}$ \\
\hline & & & 20000 & $4.300^{\circ}$ \\
\hline & & & 20000 & $4.300^{2}=$ \\
\hline $12 / 05 / 95 \cdot 12$ & & & 20000 & $4,300^{\circ}$ \\
\hline $\begin{array}{l}12 / 13 / 95.12 / 20 / 95 \\
12 / 20 / 95.12 / 27 / 95\end{array}$ & 568 & 0 & $\begin{array}{l}20000 \\
20000\end{array}$ & $4300^{\circ} 0^{\circ}$ \\
\hline $12 / 27 / 95.01 / 03 / 96$ & 1060 & 2.0 & 20000 & $4300^{\circ}$ \\
\hline
\end{tabular}

STHEOL KEY: * Concentration exceeds Harning Level. 


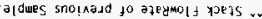
$2 \mathrm{abed}$

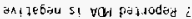

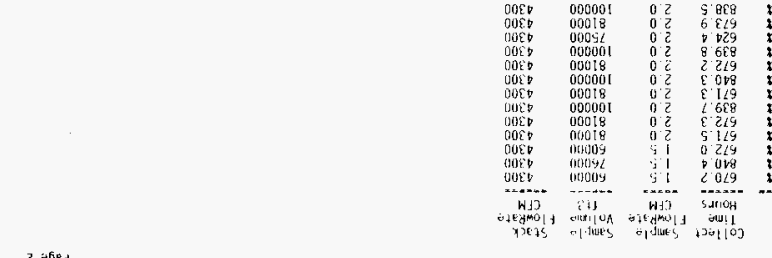

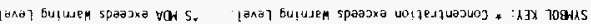

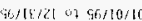

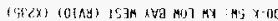

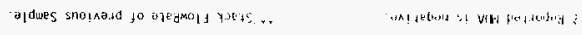

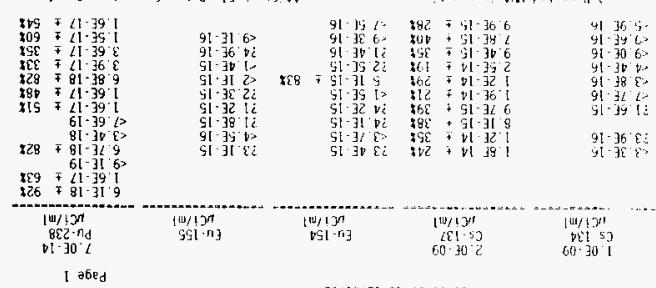

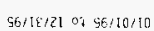

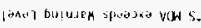

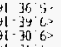

1. 34 行

ใ. $36 \varepsilon^{\circ} \varepsilon$

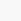

\begin{tabular}{|c|c|}
\hline 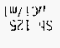 & $\begin{array}{l}1 w 1100 \\
901-n y \\
60-30 \text { ह }\end{array}$ \\
\hline
\end{tabular}

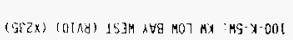

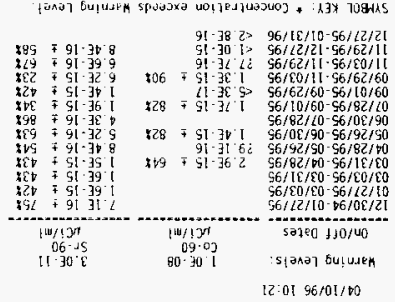




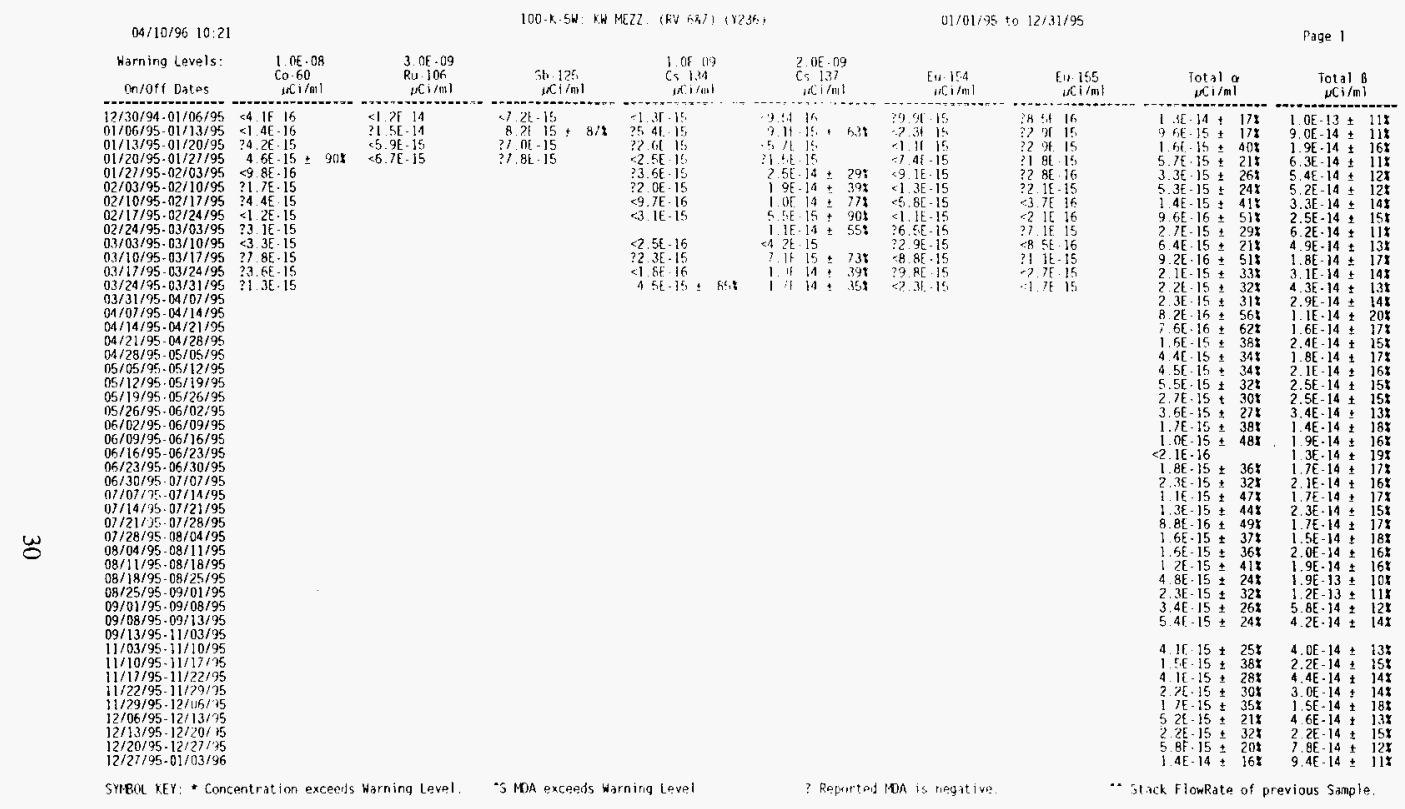



诘U

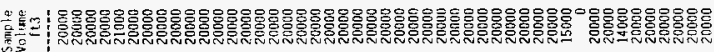
造: 然:

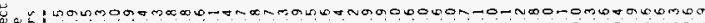

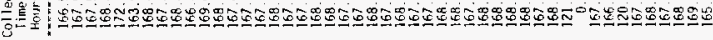

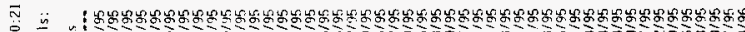
与 \%

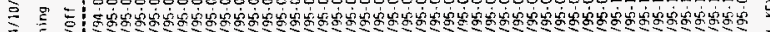
存

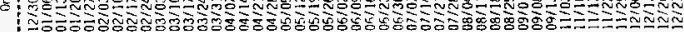




\begin{tabular}{|c|c|c|c|c|c|c|c|c|c|}
\hline \multirow{2}{*}{$\begin{array}{l}\text { 04/10/96 10:21 } \\
\text { Warning Levels: } \\
\text { Onroff Dates }\end{array}$} & \multirow[b]{2}{*}{$\begin{array}{l}1.0 E \cdot 08 \\
C 0.60 \\
\mid c i / m !\end{array}$} & \multicolumn{4}{|c|}{ 100-K. - SW : KH MEZZ (PV 627) $(x 236)$} & \multicolumn{2}{|c|}{ u1/01/95 to $12 / 31 / 95$} & \multirow{2}{*}{\multicolumn{2}{|c|}{$\begin{array}{l}\text { Page } 1 \\
70 \in-14 \\
\begin{array}{c}7 \mathrm{u}-238 \\
\mathrm{iCi} / \mathrm{ml}\end{array}\end{array}$}} \\
\hline & & 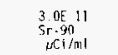 & 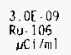 & 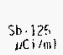 & 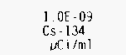 & 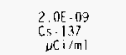 & 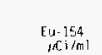 & & \\
\hline 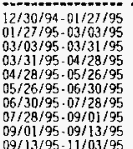 & 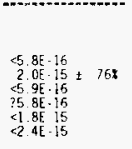 & 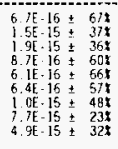 & & & 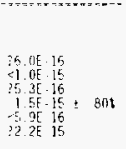 & 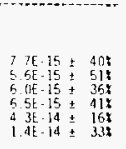 & 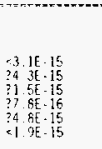 & $\begin{array}{l}-2.5 \mathrm{E}-15 \\
? 3.3 \mathrm{E}-15 \\
83.6 \mathrm{E}-17 \\
22.6 \mathrm{E}-15 \\
29.1 \mathrm{E}-16\end{array}$ & 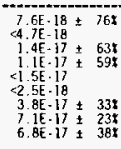 \\
\hline $\begin{array}{l}11 / 303 / 95-11 / 295 \\
11 / 29 / 95-12 / 27 / 95 \\
112 / 27 / 95.01 / 31 / 96\end{array}$ & 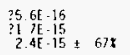 & $\begin{array}{l}6.86 \cdot 16 \pm 678 \\
1.58 \cdot 15 \div 428\end{array}$ & & & 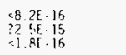 & 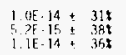 & 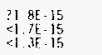 & 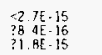 & 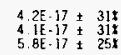 \\
\hline
\end{tabular}

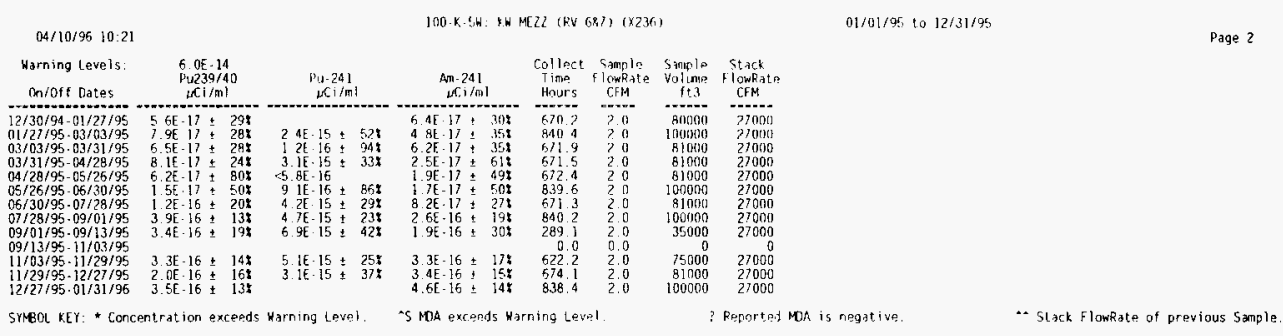




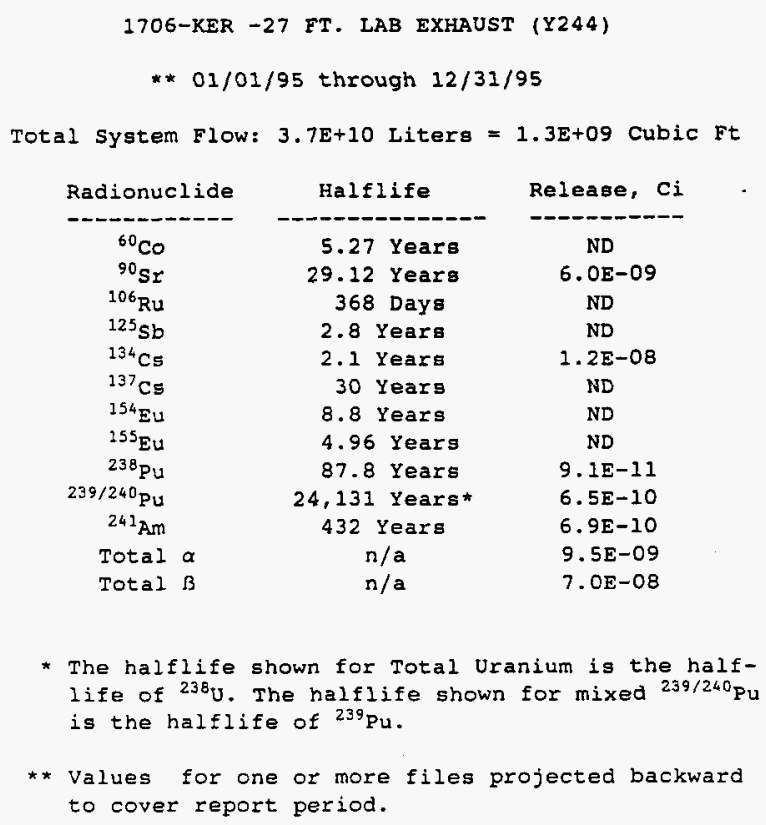


04/10/95 10:21

100-K.KER: 1705-KER -27 ft 1.3t Exhayet (12244)

$01 / 01 / 95$ in $12 / 31 / 95$

\begin{tabular}{|c|c|c|c|c|c|c|c|c|c|}
\hline $\begin{array}{l}\text { Haming Levels: } \\
\text { On/off Dates }\end{array}$ & 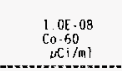 & $\begin{array}{l}3 . n e-11 \\
\text { sr. } 90 \\
\mu C i / m 1\end{array}$ & $\begin{array}{l}3.0 E-09 \\
R u-106 \\
\text { Reifilal }\end{array}$ & $\begin{array}{c}5 b-125 \\
{[c i / m]} \\
0<1\end{array}$ & 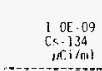 & 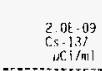 & 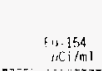 & $\begin{array}{l}\text { Eu. } 155 \\
p C \mid / m 1\end{array}$ & $\begin{array}{r}7.0 \mathrm{E}-14 \\
\mathrm{Pu}^{2} 238 \\
\nu \mathrm{C} / \mathrm{m} / \mathrm{m}\end{array}$ \\
\hline $\begin{array}{l}12 / 02 / 94-01 / 05 / 95 \\
01 / 06 / 95-02 / 10 / 95 \\
02 / 10 / 95-03 / 03 / 95 \\
03 / 03 / 95-04 / 07 / 95 \\
04 / 07 / 95-05 / 05 / 95 \\
05 / 05 / 95-06 / 02 / 95 \\
06 / 02 / 95-07 / 07 / 95 \\
07 / 0 / 95-08 / 04 / 95 \\
08 / 04 / 95-09 / 01 / 95 \\
09 / 01 / 95-10 / 05 / 95 \\
10 / 06 / 75-11 / 03 / 95 \\
11 / 03 / 95-12 / 101 / 95 \\
12 / 01 / 95-01 / 08 / 96\end{array}$ & $\begin{array}{l}7.8 E 16 \pm 908 \\
? 3.2 E-16 \\
3.4 E-17 \\
? 2.2 E-16\end{array}$ & 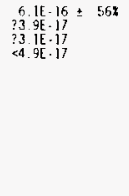 & 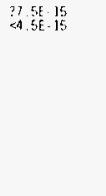 & $\begin{array}{l}? 7.16 \\
? 1.6 \mathrm{t} \cdot 16\end{array}$ & 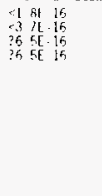 & $\begin{array}{l}10 \mathrm{l} \cdot 15 \\
? 1.8 \mathrm{E}-16 \\
\text { ?1 } 9 \mathrm{E} \cdot 16 \\
? .7 \mathrm{E} \cdot 17\end{array}$ & 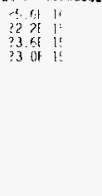 & $\begin{array}{l}3.5 \mathrm{k} \cdot 16 \\
? 2.4 \mathrm{E} \cdot 16 \\
\text { ?1.1E-15 } \\
? 2.1 \mathrm{E}-16\end{array}$ & $\begin{array}{l}? 3.8 E-19 \\
? 36.9 E-19 \\
55.4 E-19 \\
? 2.8 E-19\end{array}$ \\
\hline
\end{tabular}

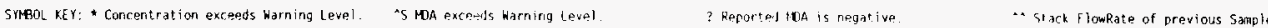
04/10/96 10:21
100-K-KER: 17 lif -KFR -27 f: Lab Exhatus? (Y201)
$0 / / 01 / 75$ to $12 / 31 / 45$
Page 2

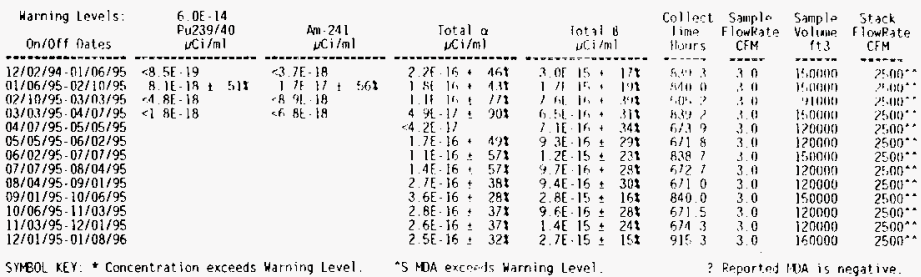


$04 / 10 / 96 \quad 10: 21$

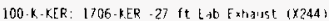

$01 / 01 / 95$ :0 12/31/45

\begin{tabular}{|c|c|c|c|c|c|c|c|c|c|}
\hline $\begin{array}{l}\text { Warning levels: } \\
\text { On/orf Dates }\end{array}$ & $\begin{array}{l}\text { 8. } 0 \mathrm{E} \cdot 11 \\
\mathrm{Co} \cdot 60 \\
\text { icim? }\end{array}$ & 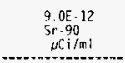 & $\begin{array}{c}3.0[-11 \\
R_{u}-105 \\
\mu C i / m !\end{array}$ & $\begin{array}{r}1.0 E-09 \\
s b \cdot 125 \\
y C i m !\end{array}$ & 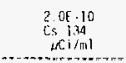 & $\begin{array}{l}\text { a. } D f-10 \\
\text { Cs-137 } \\
\text { icisis }\end{array}$ & 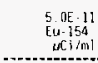 & 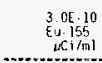 & 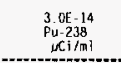 \\
\hline $\begin{array}{l}04 / 97 / 95-07 / 07 / 95 \\
07 / 07 / 95-10 / 06 / 95 \\
10 / 06 / 95-01 / 08 / 96\end{array}$ & $\begin{array}{l}2.1 \mathrm{E}-16 \\
2.8 \mathrm{E} \cdot 16 \\
<4.0 \mathrm{E}-16\end{array}$ & 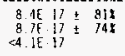 & & & $\begin{array}{l}2.9 E-16 \pm 86 ! \\
? 12 E-16 \\
5.0 E-16 \pm 5\end{array}$ & $\begin{array}{rl}3 & 11-16 \\
2.8[-16 \\
31.3 t-17\end{array}$ & $\begin{array}{ll}-486 \\
-4\end{array}$ & $\begin{array}{l}25 \mathrm{E}-16 \\
? 3 \mathrm{BE}-16 \\
? 7 \mathrm{E}-17\end{array}$ & $\begin{array}{r}2.9 E-18 \pm 58 t \\
<.0 E-19=98 \\
1.0 E-18 \pm 918\end{array}$ \\
\hline
\end{tabular}

SMEOL KEY: * Concentration exceeds Warning Level.

100-K.KER: 17OG. -ER . 27 it Lat Exhaust (X244)

$01 / 01 / 95$ to $12 / 31 / 95$

Fage 2

\begin{tabular}{|c|c|c|c|c|c|c|}
\hline darnin & 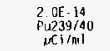 & 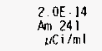 & $\begin{array}{l}\text { Collert } \\
\text { Thier } \\
\text { Hours }\end{array}$ & $\begin{array}{c}\text { Samplo } \\
\text { Fiowlide } \\
\text { C.FM }\end{array}$ & $\begin{array}{c}\text { simncle } \\
\text { volume } \\
\text { rita }\end{array}$ & $\begin{array}{c}\text { Stack } \\
\text { rlawpites } \\
\text { CFM }\end{array}$ \\
\hline & $=$ & & & & & \\
\hline
\end{tabular}

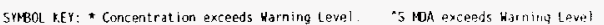


WHC ENVIRONMENTAL RELEASE SUMMARY

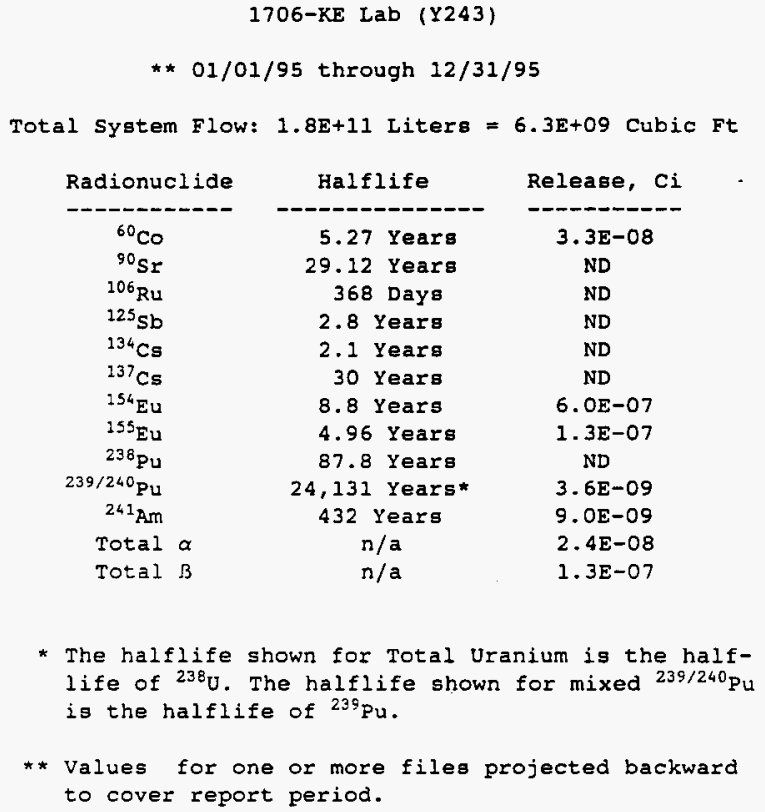

* The halflife shown for Total Uranium is the halflife of ${ }^{238} \mathrm{U}$. The halflife shown for mixed ${ }^{239 / 240} \mathrm{Pu}$ is the halflife of ${ }^{239} \mathrm{Pu}$.

** Values for one or more files projected backward to cover report period. 


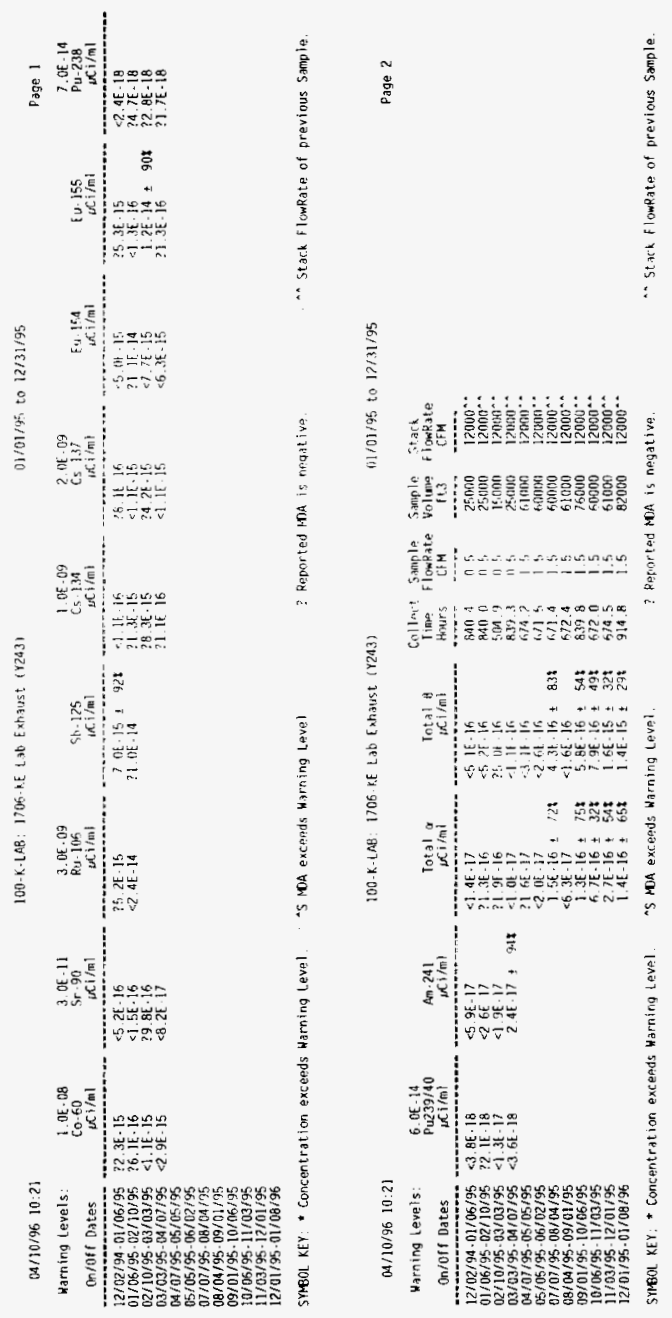




\begin{tabular}{|c|c|c|c|c|c|c|c|c|c|}
\hline \multicolumn{3}{|l|}{$04 / 10 / 96 \quad 10.21$} & \multicolumn{3}{|c|}{ 100-K-LAB: 1706-RE Lat [xhinust \& $\times 743]$} & \multicolumn{2}{|c|}{$01 / 01 / 95$ to $1.3 / 31 / 95$} & \multicolumn{2}{|r|}{ Page 1} \\
\hline $\begin{array}{l}\text { Karning Levels: } \\
\text { Onforf Dates }\end{array}$ & 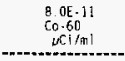 & $\begin{array}{l}\text { 9. } 0 \mathrm{E}-12 \\
5 \mathrm{r}-90 \\
\mathrm{wCi} / \mathrm{ml}\end{array}$ & 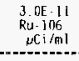 & 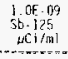 & 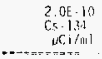 & 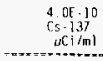 & 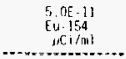 & $\begin{array}{l}\text { 3. } 0 E-10 \\
\text { Eu-155 } \\
\text { LCitm! }\end{array}$ & 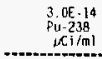 \\
\hline $\begin{array}{l}04 / 07 / 95 \cdot 06 / 02 / 95 \\
07 / 707 / 95-10 / 05 / 95 \\
10 / 06 / 95-01 / 08 / 96\end{array}$ & $\begin{array}{l}\text { 4. 9E-16 } \\
? 8.7 \mathrm{E}-16 \\
6.9 \mathrm{E}-16 \pm 908\end{array}$ & $\begin{array}{l}? 3 \cdot 1 \mathrm{E}-1 ? \\
<5 \mathrm{BE} \cdot 11 \\
? 7 \cdot \mathrm{ZE} \cdot 18\end{array}$ & & & $\begin{array}{llll}? 2 & 8 \mathrm{~F} & 15 \\
? 2 & \text { If } & 17 \\
? 3 & \text { it } & -17\end{array}$ & $\begin{array}{r}5.17 \\
<3.45 \cdot 15 \\
<2.8 \mathrm{E} \cdot 16\end{array}$ & 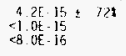 & $\begin{array}{lll}1 & 15 & 15 \\
1 & 2 E & -16 \\
5 & 8 t & -17\end{array}$ & $\begin{array}{l}\text { c]. } 3 E-18 \\
\text { c1 䜣-18 } \\
\text { c1. } 4 \mathrm{E}-18\end{array}$ \\
\hline
\end{tabular}

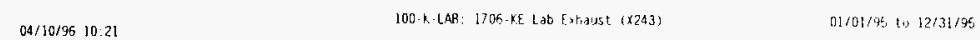

\begin{tabular}{|c|c|c|c|c|c|c|}
\hline $\begin{array}{l}\text { Harning Levels: } \\
\text { On/off Dates }\end{array}$ & 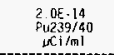 & $\begin{array}{l}2.05 \cdot 14 \\
\sin 241 \\
1 \times i / m !\end{array}$ & $\begin{array}{l}\text { Collect } \\
\text { Time } \\
\text { Hours }\end{array}$ & $\begin{array}{l}\text { Sample } \\
\text { Flomknte } \\
\text { CFIM }\end{array}$ & $\begin{array}{l}\text { S.mplo } \\
\text { volume } \\
\text { vit. }\end{array}$ & 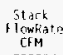 \\
\hline $\begin{array}{l}04 / 07 / 95-06 / 02 / 95 \\
01 / 07 / 95.10 / 06 / 95 \\
10 / 06 / 95.01 / 08 / 96\end{array}$ & $\begin{array}{l}\text { SE. }-17 \pm 418 \\
1.17 \pm 438 \\
1.71 .17 \pm 318\end{array}$ & $\begin{array}{l}\text { IE- }-17+36 \\
3 E-18 \pm 648 \\
3 E-18 \pm 585\end{array}$ & $\begin{array}{l}13451 \\
2183.6 \\
22613\end{array}$ & $\begin{array}{l}5 \\
5 \\
1.5\end{array}$ & $\begin{array}{l}12001000 \\
2005000 \\
200000\end{array}$ & $\begin{array}{l}120019 \\
1210691 \\
12009\end{array}$ \\
\hline
\end{tabular}

Page 2

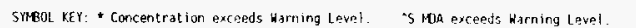

? Feporteil ton is regative

.. Stack FlowRite of previous Sample. 
WHC-SD-EN-TI-308 Rev.0

$2.3100-\mathrm{N}$ Area 
1AN116: 116-N STACK PARTICULATE (X211, Y211, Y212, Y213)

$$
01 / 01 / 95 \text { through } 12 / 31 / 95 \star \star *
$$

Total system Flow: $1.6 \mathrm{E}+12$ Liters $=5.8 \mathrm{E}+10$ Cubie Ft

The following factor(s) has (or have) been applied to the radionuclide concentrations used in the calculation of this release to account for sampling filter efficiency, line and bend loss, and humidity. The concentrations vary inversely with the size of the factor(s).

\section{Particulates:}

${ }^{60} \mathrm{Co}$
${ }^{90} \mathrm{Sr}$
$106 \mathrm{Ru}$
$125 \mathrm{Sb}$
$134 \mathrm{Cs}$
${ }^{137} \mathrm{Cs}$
${ }^{154} \mathrm{Eu}$
${ }^{155} \mathrm{Eu}$
$238 \mathrm{Pu}$
$239 / 2{ }^{40} \mathrm{Pu}$
$241 \mathrm{Am}$
Total a
Total $B$

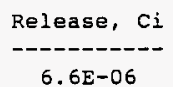

ND

ND

ND

ND

ND

ND

ND

ND

5. $8 \mathrm{E}-08$

4. $2 E-08$

5. 3E-07

9. $5 E-06$
0.73

$\begin{array}{cc}\text { Concentrations, } \mu \mathrm{Ci} / \mathrm{ml} \\ \text { Average } & \text { Peak } \\ ---1 & -1-- \\ 2.9 \mathrm{E}-15 & 2.5 \mathrm{E}-14 \\ <9.3 \mathrm{E}-18 & \mathrm{n} / \mathrm{a} \\ <7.7 \mathrm{E}-15 & \mathrm{n} / \mathrm{a} \\ <1.1 \mathrm{E}-15 & \mathrm{n} / \mathrm{a} \\ ? 1.8 \mathrm{E}-16 & \mathrm{n} / \mathrm{a} \\ -2.5 \mathrm{E}-16 & 3.5 \mathrm{E}-15 \\ <1.3 \mathrm{E}-15 & \mathrm{n} / \mathrm{a} \\ <1.3 \mathrm{E}-15 & \mathrm{n} / \mathrm{a} \\ <9.8 \mathrm{E}-19 & \mathrm{n} / \mathrm{a} \\ 2.6 \mathrm{E}-17 & 3.2 \mathrm{E}-17 \\ 1.9 \mathrm{E}-17 & 2.2 \mathrm{E}-17 \\ 2.4 \mathrm{E}-16 & 2.8 \mathrm{E}-15 \\ 4.2 \mathrm{E}-15 & 4.4 \mathrm{E}-14\end{array}$

** Values projected for period $07 / 21 / 95$ to $12 / 31 / 95$.

? A question mark ("?") indicates an average concentration less than a negative value. 
04/10/96 10:21

1AN116: 116-H STACK PARTICULATE (Y211)

(1)/01/25 to [2/3]/95

Page 1

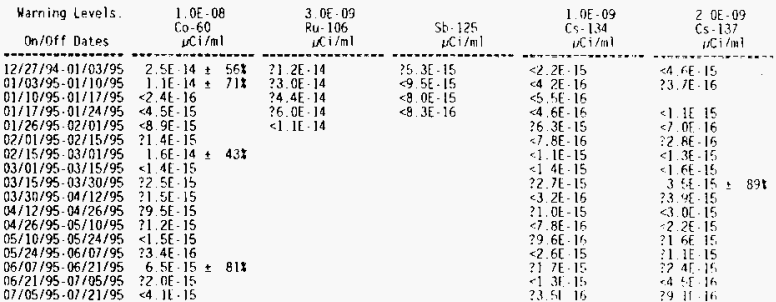

5YMEOL KEY. * Concentration exceads Harning Level

04/10/96 10:21

5 Mid exceeds warning Lavol

IAN1 16. 116-1 STACK PARTICULATS (Y211)

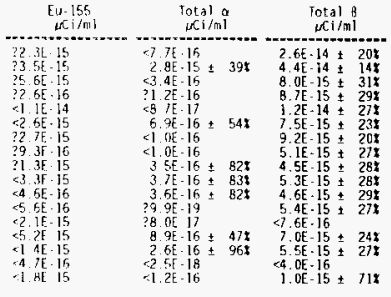

an stack flowfate of previous 5 mople 

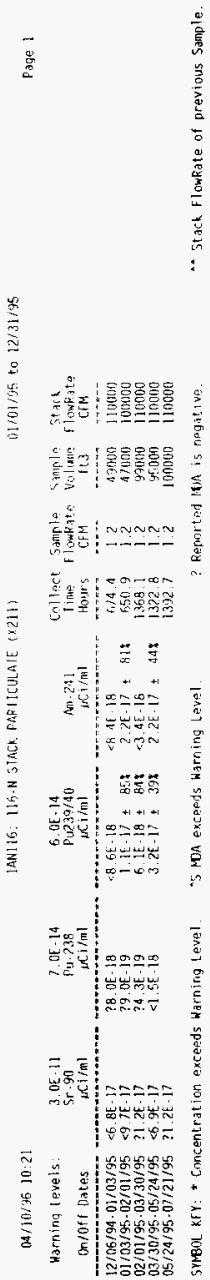


\section{WHC-SD-EN-TI-308 Rev.0}

WHC ENVIRONMENTAL RELEASE SUMMARY

1ANO5DCN: 14, DECON FACIIITY PARTICULATE (Y259, Y260)

$01 / 01 / 95$ through $12 / 31 / 95$

Total system Flow: $9.5 E+10$ Liters $=3.4 E+09$ Cubic Ft

The following factor(s) has (or have) been applied to the radionuclide concentrations used in the calculation of this release to account for sampling filter efficiency, line and bend loss, and humidity. The concentrations vary inversely with the size of the factor(s).

$$
\text { Particulates: } \quad 0.73
$$

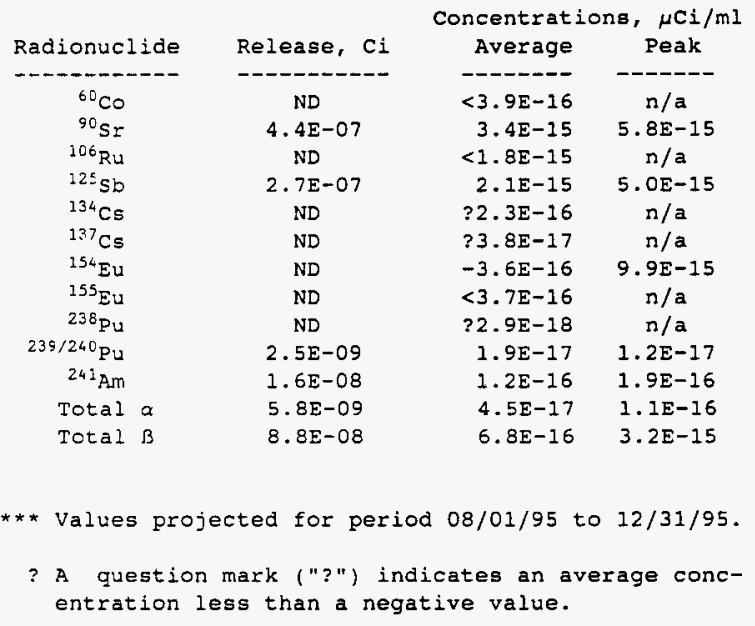




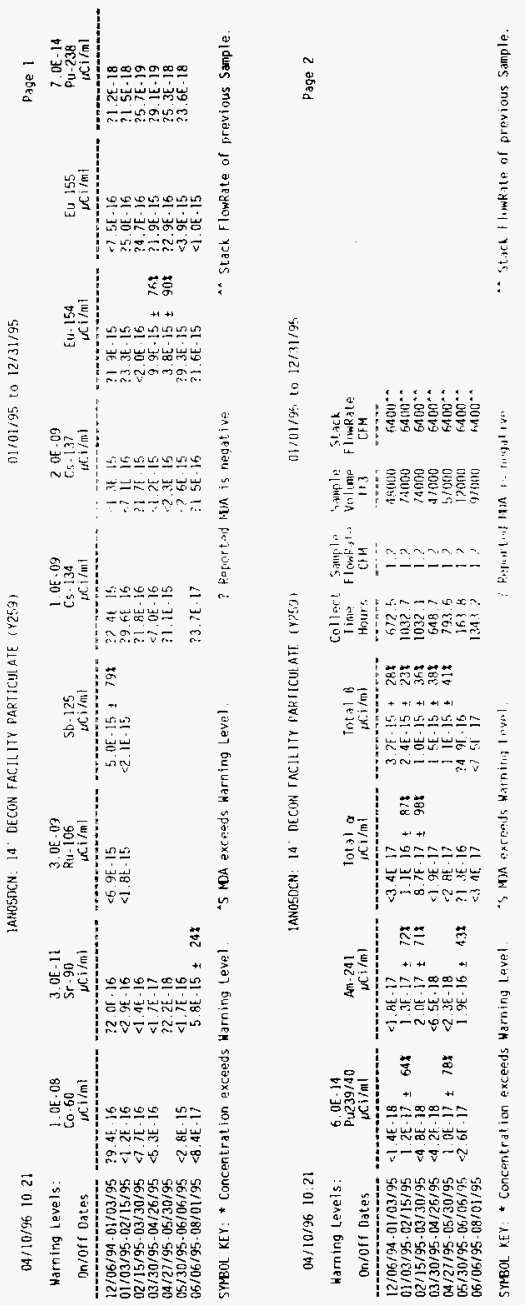




\section{WHC-SD-EN-TI-308 Rev.0}

IANO7ROOF: 107-N EXHAUST PARTICULATE (Y265, Y 266)

$$
01 / 01 / 95 \text { through } 12 / 31 / 95 * * *
$$

Total system Flow: $1.1 \mathrm{E}+11$ Liters $=3.8 \mathrm{E}+09$ cubic Ft

The following factor(s) has (or have) been applied to the radionuclide concentrations used in the calculation of this release to account for sampling filter efficiency, line and bend loss, and humidity. The concentrations vary inversely with the size of the factor $(\mathbf{s})$.

$$
\text { Particulates: } \quad 0.73
$$

\begin{tabular}{cccc} 
& & \multicolumn{2}{c}{ Concentrations, $\mu \mathrm{Ci} / \mathrm{ml}$} \\
Radionuclide & Release, Ci & Average & Peak \\
\hline${ }^{60} \mathrm{Co}$ & $1.0 \mathrm{E}-07$ & $6.9 \mathrm{E}-16$ & $1.4 \mathrm{E}-15$ \\
${ }^{90} \mathrm{Sr}$ & $1.5 \mathrm{E}-07$ & $9.8 \mathrm{E}-16$ & $1.7 \mathrm{E}-15$ \\
${ }^{106} \mathrm{Ru}$ & $\mathrm{ND}$ & $<2.7 \mathrm{E}-15$ & $\mathrm{n} / \mathrm{a}$ \\
${ }^{125} \mathrm{Sb}$ & $\mathrm{ND}$ & $? 1.2 \mathrm{E}-15$ & $\mathrm{n} / \mathrm{a}$ \\
${ }^{134} \mathrm{Cs}$ & $\mathrm{ND}$ & $<5.0 \mathrm{E}-16$ & $\mathrm{n} / \mathrm{a}$ \\
${ }^{137} \mathrm{Cs}$ & $\mathrm{ND}$ & $<2.0 \mathrm{E}-16$ & $\mathrm{n} / \mathrm{a}$ \\
${ }^{154} \mathrm{Eu}$ & $\mathrm{ND}$ & $<2.0 \mathrm{E}-16$ & $\mathrm{n} / \mathrm{a}$ \\
${ }^{155} \mathrm{Eu}$ & $2.0 \mathrm{E}-07$ & $1.4 \mathrm{E}-15$ & $2.1 \mathrm{E}-15$ \\
$238 \mathrm{Pu}$ & $\mathrm{ND}$ & $<3.1 \mathrm{E}-18$ & $\mathrm{n} / \mathrm{a}$ \\
$239 / 240 \mathrm{Pu}$ & $2.3 \mathrm{E}-09$ & $1.6 \mathrm{E}-17$ & $1.5 \mathrm{E}-17$ \\
$241 \mathrm{Am}$ & $3.6 \mathrm{E}-09$ & $2.4 \mathrm{E}-17$ & $1.3 \mathrm{E}-17$ \\
Total $\alpha$ & $3.1 \mathrm{E}-09$ & $2.1 \mathrm{E}-17$ & $1.2 \mathrm{E}-16$ \\
Total B & $9.5 \mathrm{E}-08$ & $6.4 \mathrm{E}-16$ & $6.6 \mathrm{E}-15$ \\
& & & \\
*** & & &
\end{tabular}




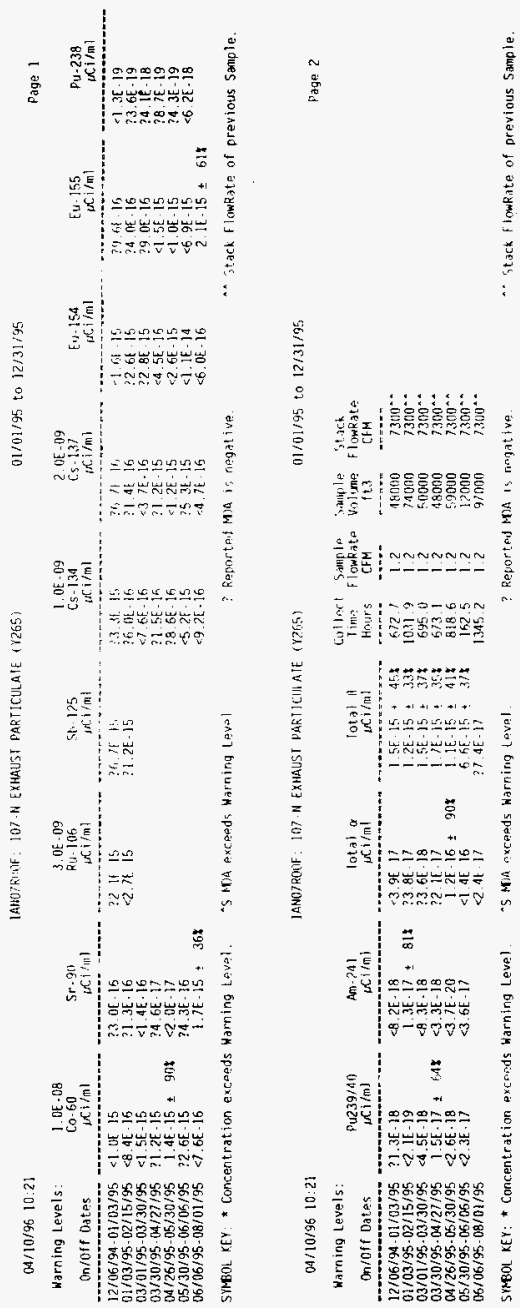


WHC-SD-EN-TI-308 Rev.0

2.4 200 East Area

2.4.1 PUREX 


\section{WHC-SD-EN-TI-308 Rev.0}

$08 / 01 / 96$

ENVIRONMENTAL RELEASE SUMMARY REPORT

291-A-1: PUREX MAIN STACK (A511, A552, A566, A940, X552)

$01 / 01 / 95$ through $12 / 31 / 95 \star \star \star$

Total system Flow: $1.6 \mathrm{E}+12$ Liters $=5.5 \mathrm{E}+10$ Cubic Ft

The following factor(s) has (or have) been applied to the radionuclide concentrations used in the calculation of this release to account for sampling filter efficiency, line and bend loss, and humidity. The concentrations vary inversely with the size of the factor $(s)$.

\begin{tabular}{|c|c|c|c|}
\hline \multicolumn{2}{|c|}{$\begin{array}{l}\text { Particulates: } \\
\text { Halogens: }\end{array}$} & \multicolumn{2}{|l|}{$\begin{array}{l}0.52 \\
0.87\end{array}$} \\
\hline Radionuclide & Release, $\mathrm{Ci}$ & $\begin{array}{c}\text { Concentrati } \\
\text { Average }\end{array}$ & 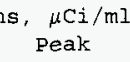 \\
\hline$--------\cdots$ & $--\ldots------$ & -------- & ------ \\
\hline${ }^{90} \mathrm{Sr}$ & $4.2 \mathrm{E}-05$ & $1.4 \mathrm{E}-14$ & $2.8 E-14$ \\
\hline${ }^{103} \mathrm{Ru}$ & ND & $<1 . O E-14$ & $\mathrm{n} / \mathrm{a}$ \\
\hline${ }^{106} \mathrm{Ru}$ & ND & $-2.6 \mathrm{E}-15$ & 1. $6 \mathrm{E}-14$ \\
\hline${ }^{113} \mathrm{Sn}$ & ND & $-2.7 E-16$ & 1. $9 E-15$ \\
\hline${ }^{125} \mathrm{Sb}$ & $7.4 E-06$ & $2.7 E-15$ & $6.0 E-15$ \\
\hline${ }^{129} \mathrm{I}$ & 8. $6 E-03$ & $4.8 E-I 2$ & 2. $9 E-11$ \\
\hline${ }^{131} \mathrm{I}$ & ND & $<3.3 E-14$ & $n / a$ \\
\hline${ }^{134} \mathrm{Cs}$ & $\mathrm{ND}$ & $-2.2 E-17$ & $2.4 \mathrm{E}-17$ \\
\hline${ }^{137} \mathrm{Cs}$ & 1. $0 \mathrm{E}-04$ & $3.7 E-14$ & $6.4 E-14$ \\
\hline${ }^{152}$ Eu & $3.5 E-07$ & 1. $2 \mathrm{E}-16$ & $2.5 E-16$ \\
\hline${ }^{154} \mathrm{Eu}$ & $3.2 E-07$ & 1. $1 E-16$ & 1. $9 \mathrm{E}-16$ \\
\hline${ }^{155} \mathrm{Eu}$ & $9.4 \mathrm{E}-08$ & $3.2 \mathrm{E}-17$ & $1.5 \mathrm{E}-16$ \\
\hline${ }^{238} \mathrm{Pu}$ & $5.7 \mathrm{E}-07$ & 1. $9 E-16$ & 5. $1 E-16$ \\
\hline $239 / 240 \mathrm{Pu}$ & $4.5 E-06$ & 1. $5 E-15$ & $2.7 E-15$ \\
\hline${ }^{241} \mathrm{Pu}$ & 1. $1 E-04$ & $3.8 E-14$ & 8. $3 E-14$ \\
\hline${ }^{24} \mathrm{Am}$ & 1. $6 \mathrm{E}-05$ & 5. $2 E-15$ & 8. $6 E-15$ \\
\hline Total $\alpha$ & $1.4 \mathrm{E}-05$ & $4.6 \mathrm{E}-15$ & I. $1 \mathrm{E}-14$ \\
\hline Total is & 1. $3 E-04$ & $4 \cdot 3 E-14$ & 1. $2 \mathrm{E}-13$ \\
\hline
\end{tabular}


$06 / 21: 9611: 21$

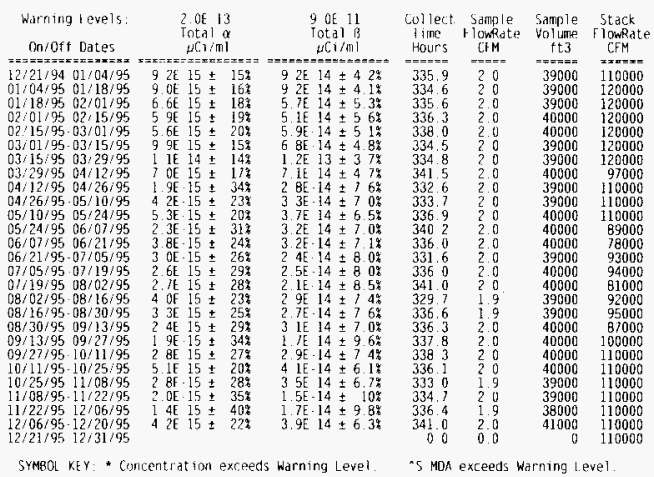

01:01/95 to 17/31/95

Page \&
291 A. 1 P1urex Ma10 5tack (A552)

$$
\begin{aligned}
& \begin{array}{l}
0 x=x=0 \\
10000
\end{array} \\
& 000 \\
& 000 \\
& 000 \\
& \begin{array}{l}
0000 \\
0000 \\
0000 \\
0000
\end{array}
\end{aligned}
$$


(th:21/9611 21

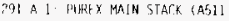

01/01/95 to $[2 / 3] / 95$ Pragn 1

\begin{tabular}{|c|c|c|c|c|c|c|c|c|c|c|c|c|}
\hline $\begin{array}{l}\text { Warring l megels. } \\
\text { On:urf Dates }\end{array}$ & & 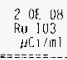 & 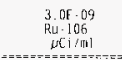 & 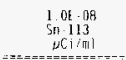 & 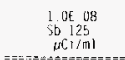 & 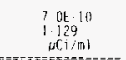 & $\begin{array}{l}4 \text { of } \mid 1) 9 \\
{[131]} \\
{[C, 1 / \mathrm{ml}}\end{array}$ & 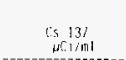 & $\begin{array}{l}\text { rollect. } \\
\text { Time } \\
\text { Hour's }\end{array}$ & $\begin{array}{l}\text { Solnple } \\
\text { Flowkati! } \\
\text { crit }\end{array}$ & $\begin{array}{l}\text { Sample } \\
\text { volure } \\
\mathrm{ft} 3\end{array}$ & $\begin{array}{l}\text { St ack } \\
\text { I lowR } \\
\text { CFM }\end{array}$ \\
\hline 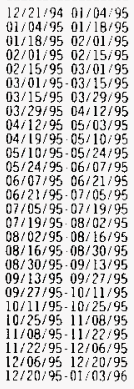 & & & 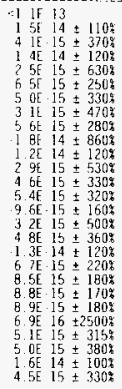 & 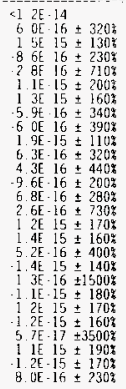 & 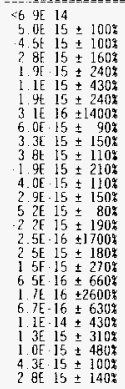 & 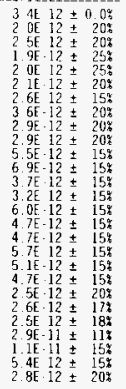 & $=33514$ & 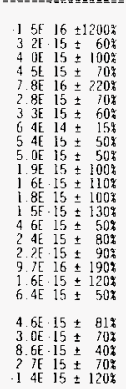 & 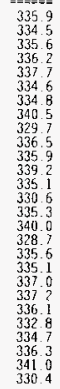 & $\begin{array}{l}==3 \\
2.0 \\
2.0 \\
2.0 \\
2.0 \\
2.0 \\
2.0 \\
2.0 \\
2.0 \\
2.0 \\
2.0 \\
2.0 \\
2.0 \\
2.0 \\
2.0 \\
2.0 \\
2.0 \\
2.0 \\
2.0 \\
2.0 \\
2.0 \\
2.0 \\
2.0 \\
1.9 \\
2.0 \\
1.7 \\
2.0 \\
2.2\end{array}$ & $\begin{array}{l}39=0 \\
39000 \\
40000 \\
40000 \\
40000 \\
40000 \\
40000 \\
40000 \\
41000 \\
40000 \\
40000 \\
410000 \\
41000 \\
40000 \\
400000 \\
400000 \\
410000 \\
39000 \\
40000 \\
40000 \\
40000 \\
40000 \\
40000 \\
38000 \\
46000 \\
34000 \\
41000 \\
43000\end{array}$ & 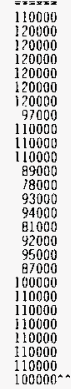 \\
\hline
\end{tabular}




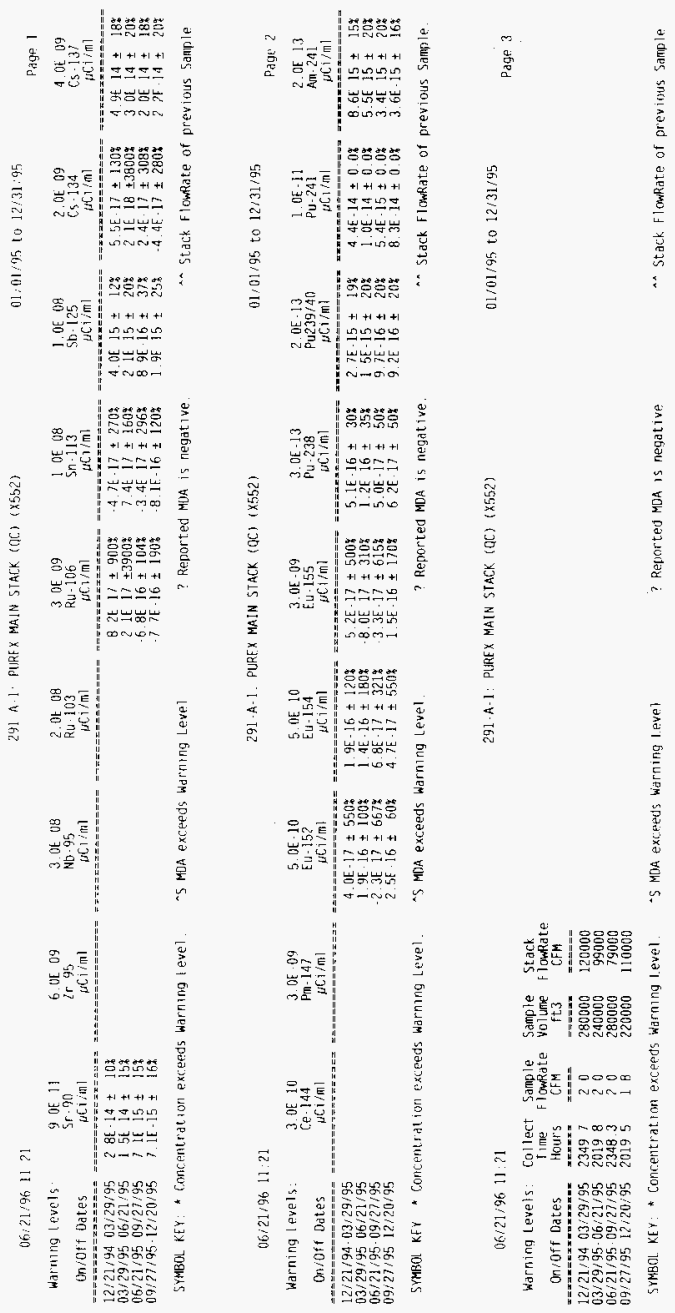


WHC-SD-EN-TI-308 Rev.0

$08 / 01 / 96$

ENVIRONMENTAL RELEASE SUMMARY REPORT

296-A-1: PUREX L, N, Q CELLS \& PR ROOM (A540, X540)

$01 / 01 / 95$ through $12 / 31 / 95$

Total System Flow: $5.9 E+10$ Liters $=2.1 \mathrm{E}+09$ Cubic Ft

The following factor(s) has (or have) been applied to the radionuclide concentrations used in the calculation of this release to account for sampling filter efficiency, line and bend loss, and humidity. The concentrations vary inversely with the size of the factor $(s)$.

Particulates :

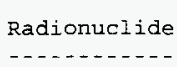

${ }^{238} \mathrm{pu}$

$239 / 240 \mathrm{Pu}$

${ }^{241} \mathrm{Pu}$

$241 \mathrm{Am}$

Total $\alpha$

Total B

Release, $\mathrm{Ci}$
$1.1 \mathrm{E}-07$
$1.8 \mathrm{E}-06$
$9.4 \mathrm{E}-06$
$5.4 \mathrm{E}-07$
$1.2 \mathrm{E}-06$
$1.3 \mathrm{E}-07$

0.73

\begin{tabular}{cc} 
Concentrations, $\begin{array}{c}\mu \mathrm{Ci} / \mathrm{ml} \\
\text { Average }\end{array}$ & Peak \\
\hline $1.3 \mathrm{E}-15$ & $3.4 \mathrm{E}-15$ \\
$2.2 \mathrm{E}-14$ & $4.7 \mathrm{E}-14$ \\
$1.2 \mathrm{E}-13$ & $3.0 \mathrm{E}-13$ \\
$6.7 \mathrm{E}-15$ & $1.4 \mathrm{E}-14$ \\
$1.5 \mathrm{E}-14$ & $7.6 \mathrm{E}-14$ \\
$1.6 \mathrm{E}-15$ & $6.7 \mathrm{E}-15$
\end{tabular}


$06 / 21: 9611.21$

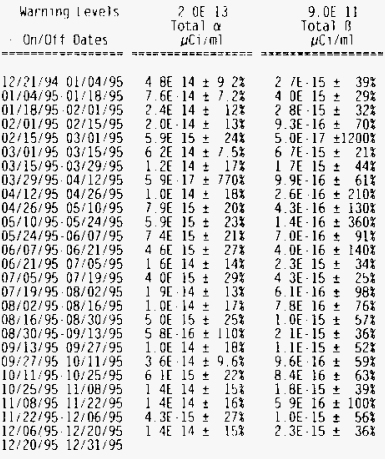

296 A I PUREX L. N. IO CEUIS \& FR PLON AA540

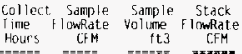

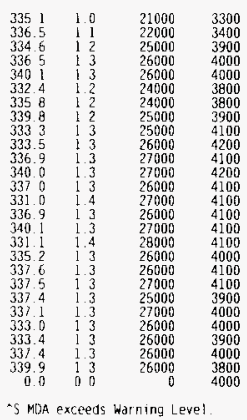

$01 / 01 / 95$ Lo $12 / 3 L / 95$

Page 1

? Reported MOA is negat ive 
WHC-SD-EN-TI-308 Rev.0
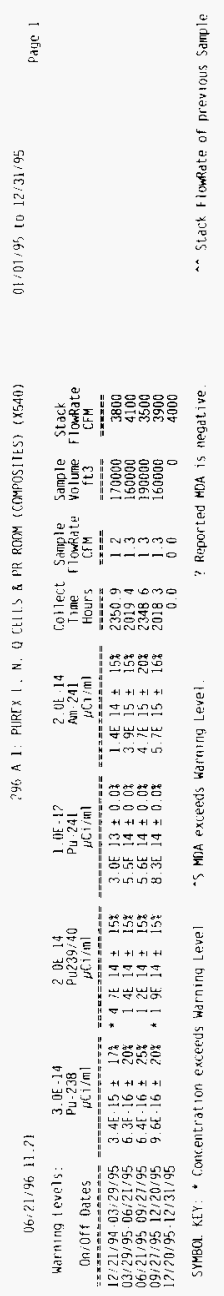
WHC-SD-EN-TI-308 Rev.0

WHC ENVIRONMENTAL, RELEASE SUMMARY

296-A-2: PUREX WEST SAMPLE GAL. HOOD EXHAUST (A.542, X542)

$01 / 01 / 95$ through $12 / 31 / 95$

Total system Flow: $3.3 \mathrm{E}+10$ Liters $=1.2 \mathrm{E}+09$ cubic $\mathrm{Ft}$

The following factor(s) has (or have) been applied to the radionuclide concentrations used in the calculation of this release to account for sampling filter efficiency, line and bend loss, and humidity. The concentrations vary inversely with the size of the factor $(s)$.

\begin{tabular}{cccc}
\multicolumn{2}{c}{ Particulates: } & 0.73 \\
Radionuclide & Release, $\mathrm{Ci}$ & $\begin{array}{c}\text { Concentrations, } \mu \mathrm{Ci} / \mathrm{ml} \\
\text { Average }\end{array}$ & Peak \\
Total $\alpha$ & $3.3 \mathrm{E}-09$ & $-1.3 \mathrm{E}-17$ & $7.3 \mathrm{E}-17$ \\
Total $B$ & $5.8 \mathrm{E}-09$ & $1.3 \mathrm{E}-16$ & $1.3 \mathrm{E}-16$
\end{tabular}


WHC-SD-EN-TI-308 Rev.0
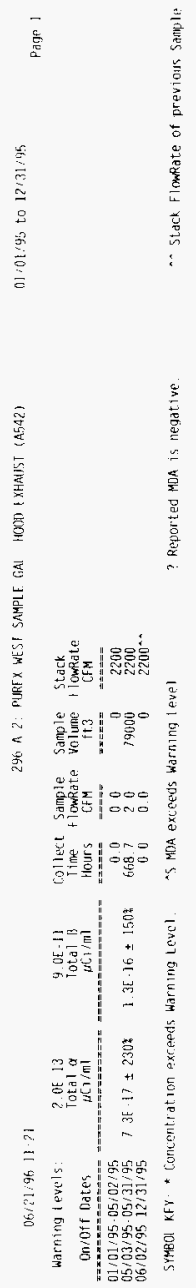


\section{WHC-SD-EN-TI-308 Rev.0}

WHC ENVIRONMENTAL RELEASE SUMMARY

296-A-3: PUREX EAST SAMPLE GAI. HOOD EXHAUST (A543)

$01 / 01 / 95$ through $12 / 31 / 95$

Total System Flow: $4.4 \mathrm{E}+10$ Liters $=1.6 \mathrm{E}+09$ Cubic Ft

The following factor(s) has (or have) been applied to the radionuclide concentrations used in the calculation of this reiease to account for sampling filter efficiency, line and bend loss, and humidity. The concentrations vary inversely with the size of the factor $(s)$.

Particulates:

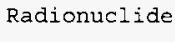

-............

Total $\alpha$

Total $B$

\author{
Release, Ci \\ -......... \\ 5. $2 \mathrm{E}-09$ \\ 1. $1 \mathrm{E}-07$
}

0.73

$\begin{array}{cc}\text { Concentrations, } \mu \mathrm{Ci} / \mathrm{ml} \\ \text { Average } & \text { Peak } \\ --------- \\ \text { 8.6E-17 } & \text { 8. } 6 \mathrm{E}-17 \\ 1.8 \mathrm{E}-15 & \text { 1. } 8 \mathrm{E}-15\end{array}$


WHC-SD-EN-TI-308 Rev.0
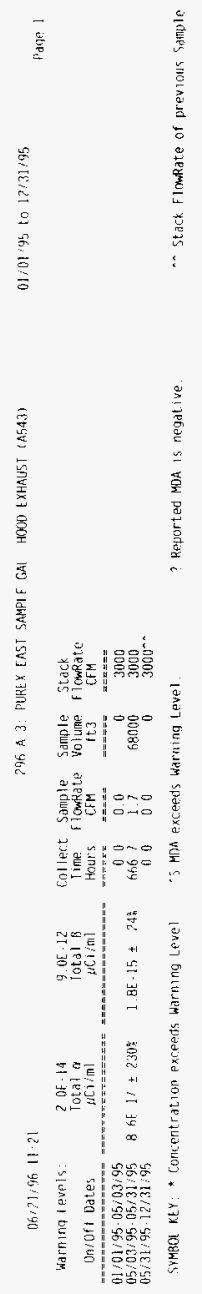


\section{WHC-SD-EN-TI-308 Rev.0}

WHC ENVIRONMENTAL RELEASE SUMMARY

$06 / 15 / 96$

296-5-A \& 296-5-B: PUREX LAB EXHAUSTERS (A545, A546)

$01 / 01 / 95$ through $12 / 31 / 95$

Total System Flow: $2.8 \mathrm{E}+11$ Liters $=9.9 \mathrm{E}+09$ Cubic Ft

\begin{tabular}{ccc} 
Radionuclide & Halflife & Release, Ci \\
\hline Total $\alpha$ & $n / a$ & $-14 \mathrm{E}-08$ \\
Total $B$ & $\mathrm{n} / \mathrm{a}$ & $8.8 \mathrm{E}-08$
\end{tabular}


WHC-SD-EN-TI-308 Rev.0
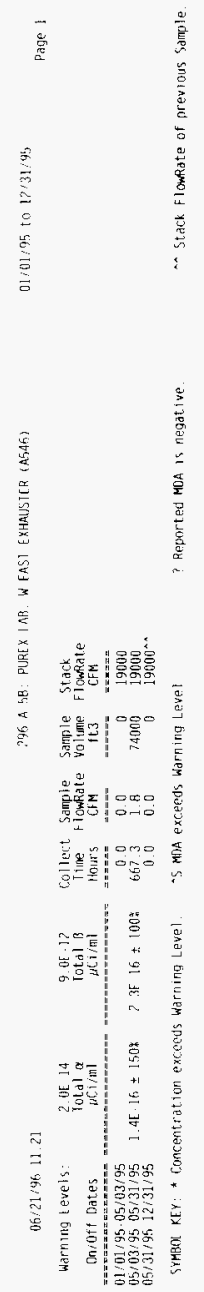


\section{WHC-SD-EN-TI-308 Rev.0}

WHC ENVIRONMENTAL RELEASE SUMMARY

296-A-6: PUREX EAST SAMPLE GAL. \& U CELI (A547, X547)

$01 / 01 / 95$ through $12 / 31 / 95$

Total System Flow: $1.8 \mathrm{E}+11$ Liters $=6.3 \mathrm{E}+09$ Cubic Ft

The following factor(s) has (or have) been applied to the radionuclide concentrations used in the calculation of this release to account for sampling filter efficiency, line and bend loss, and humidity. The concentrations vary inversely with the size of the factor $(s)$.

\begin{tabular}{cccc} 
Particulates: & 0.73 \\
Radionuclide & Release, Ci & $\begin{array}{c}\text { Concentrations, } \mu \mathrm{Ci} / \mathrm{ml} \\
\text { Average }\end{array}$ \\
\hline Peak \\
Total $\alpha$ & $\mathrm{ND}$ & $-4.3 \mathrm{E}-17$ & $-4.3 \mathrm{E}-17$ \\
Total is & $1.4 \mathrm{E}-08$ & $5.6 \mathrm{E}-17$ & $5.6 \mathrm{E}-17$
\end{tabular}


WHC-SD-EN-TI-308 Rev,0
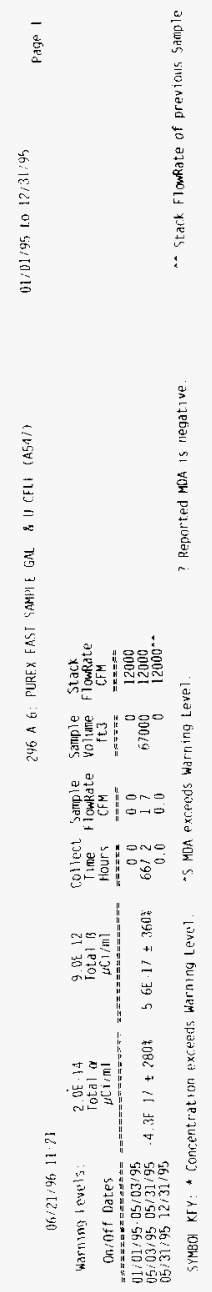


\section{WHC-SD-EN-TI-308 Rev.0}

WHC ENVIRONMENTAL, RELEASE SUMMARY

296-A-7: PUREX WEST SAMPLE GAL. \& U CELL (A548, X548)

$01 / 01 / 95$ through $12 / 31 / 95$

Total System Flow: 2.7E+11 Liters $=9.4 \mathrm{E}+09$ cubic Ft

The following factor(s) has (or have) been applied to the radionuclide concentrations used in the calculation of this release to account for sampling filter efficiency, line and bend loss, and humidity. The concentrations vary inversely with the size of the factor $(\mathrm{s})$.

Particulates:

Radionuclide

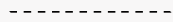

Total $\alpha$

Total $\beta$

Release, Ci
$1.2 \mathrm{E}-08$
$2.1 \mathrm{E}-07$

0.73

$\begin{array}{cc}\text { Concentrations, } \mu \mathrm{Ci} / \mathrm{ml} \\ \text { Average } & \text { Peak } \\ ------ & --- \\ 3.2 \mathrm{E}-17 & 3.2 \mathrm{E}-17 \\ 5.7 \mathrm{E}-16 & 5.7 \mathrm{E}-16\end{array}$


WHC-SD-EN-TI-308 Rev.0
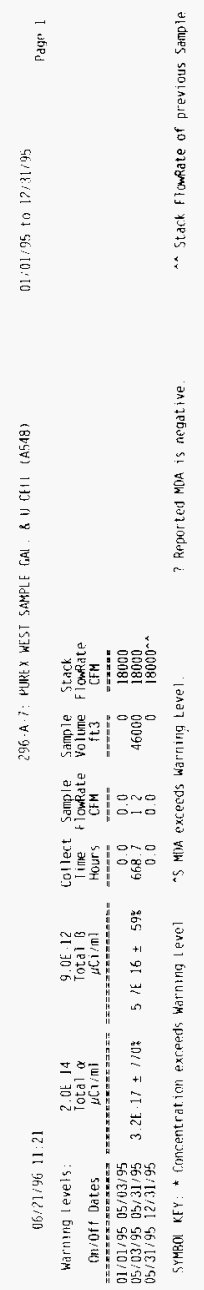


\section{WHC-SD-EN-TI-308 Rev.0}

WHC ENVIRONMENTAL RELEASE SUMMARY

296-A-8: PUREX WHITE ROOM EXHAUSTER (A549, X549)

$01 / 01 / 95$ through $12 / 31 / 95$

Total System Flow: $2.1 E+11$ Liters $=7.3 E+09$ Cubic Ft

The following factor(s) has (or have) been applied to the radionuclide concentrations used in the calculation of this release to account for sampling filter efficiency, Iine and bend loss, and humidity. The concentrations vary inversely with the size of the factor(s).

Particulates:

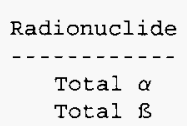

Release, $\mathrm{Ci}$
$7.1 \mathrm{E}-08$
$5.7 \mathrm{E}-07$

0.73

\begin{tabular}{cc} 
Concentrations, $\mu \mathrm{Ci} / \mathrm{ml}$ \\
Average & Peak \\
\hline$-2 .-----$ \\
2.5E-16 & $2.5 \mathrm{E}-16$ \\
2. OE-15 & 2. OE-15
\end{tabular}


WHC-SD-EN-TI-308 Rev.0
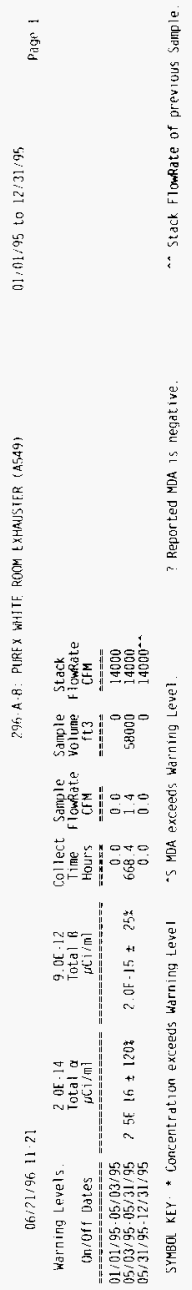
WHC-SD-EN-TI-308 Rev.0

WHC ENVIRONMENTAL RELEASE SUMMARY

296-A-10: PUREX EQUIPMENT DISPOSAL TUNNEL 2 (A550)

$01 / 01 / 95$ through $12 / 31 / 95$

Total System Flow: 1.OE+11 Liters $=3.5 E+09$ Cubic Ft

The following factor(s) has (or have) been applied to the radionuclide concentrations used in the calculation of this release to account for sampling filter efficiency, line and bend loss, and humidity. The concentrations vary inversely with the size of the factor(s).

$$
\text { Particulates: } \quad 0.73
$$

$\begin{array}{cc}\text { Radionuclide } & \text { Release, Ci } \\ \text { Total } \alpha & \text { ND } \\ \text { Total } \beta & \text { ND }\end{array}$

$\begin{array}{cc}\text { Concentrations, } \mu \mathrm{Ci} / \mathrm{ml} \\ \text { Average } & \text { Peak } \\ ------- & ---- \\ -4.8 \mathrm{E}-17 & -4.8 \mathrm{E}-17 \\ -1.6 \mathrm{E}-16 & -1.6 \mathrm{E}-16\end{array}$


WHC-SD-EN-TI-308 Rev. 0
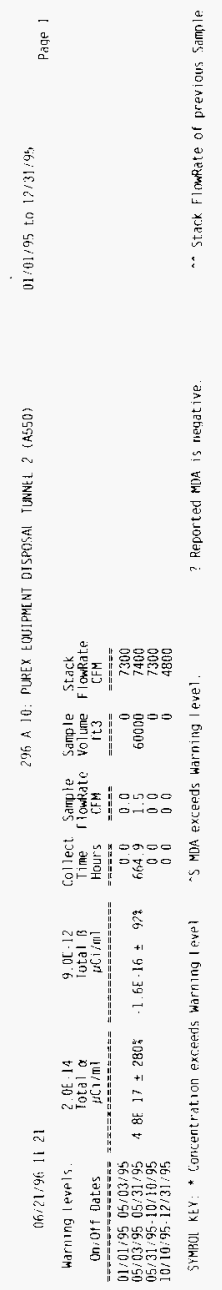


\section{WHC-SD-EN-TI-308 Rev.0}

WHC ENVIRONMENTAL RELEASE SUMMMARY

296-A-14: 293-A 'OUTBACK FACILITY' EXHAUST (A544)

$01 / 01 / 95$ through $12 / 31 / 95$

Total System Flow: $6.9 \mathrm{E}+10$ Liters $=2.4 \mathrm{E}+09$ Cubic Ft

The following factor(s) has (or have) been applied to the radionuclide concentrations used in the calculation of this release to account for sampling filter efficiency, line and bend loss, and humidity. The concentrations vary inversely with the size of the factor $(s)$.

$$
\text { Particulares: } \quad 0.73
$$

$\begin{array}{cc}\text { Radionuclide } & \text { Release, Ci } \\ \text { Total } \alpha & 1.3 E-08 \\ \text { Total } B & 2.2 E-08\end{array}$

$\begin{array}{cc}\text { Concentrations, } \mu \mathrm{Ci} / \mathrm{mI} \\ \text { Average } & \text { Peak } \\ - & ------ \\ 1.4 \mathrm{E}-16 & 1.4 \mathrm{E}-16 \\ 2.3 \mathrm{E}-16 & 2.3 \mathrm{E}-16\end{array}$


WHC-SD-EN-TI-308 Rev.0
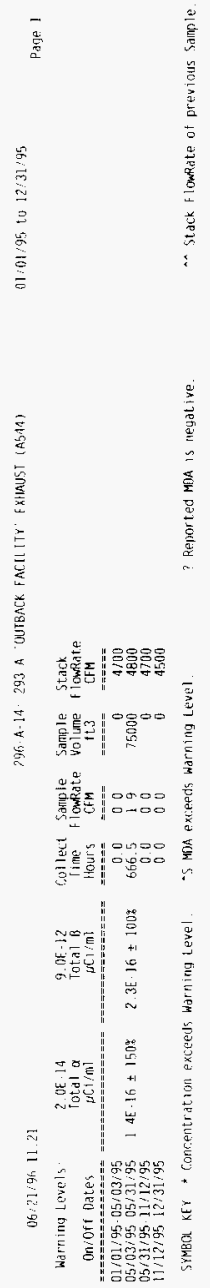
WHC-SD-EN-TI-308 Rev.0

2.4.2 B-Plant 


\section{WHC-SD-EN-TI-308 Rev.0}

WHC ENVIRONMENTAL RELEASE SUMMARY

291-B-1: 221-B BLDG \& CANYON (B-PLANT MAIN STK) (B691, X691)

$01 / 01 / 95$ through $12 / 31 / 95$

Total System Flow: $5.8 \mathrm{E}+11$ Liters $=2.0 \mathrm{E}+10$ Cubic Ft

The following factor(s) has (or have) been applied to the radionuclide concentrations used in the calculation of this release to account for sampling filter efficiency, line and bend loss, and humidity. The concentrations vary inversely with the size of the factor (s).

$$
\text { Particulates: } \quad 0.73
$$

Radionuclide
${ }^{90} \mathrm{Sr}$
${ }^{105} \mathrm{Ru}$
${ }^{113} \mathrm{Sn}$
${ }^{125} \mathrm{Sb}$
${ }^{134} \mathrm{Cs}$
${ }^{137} \mathrm{Cs}$
${ }^{152} \mathrm{Eu}$
${ }^{154} \mathrm{Eu}$
${ }^{155} \mathrm{Eu}$
${ }^{238} \mathrm{Pu}$
$239 / 240 \mathrm{Pu}$
${ }^{241} \mathrm{Am}$
Total $\alpha$
Total $\$$

Release, Ci
8. $7 \mathrm{E}-06$
ND
ND
$4.1 \mathrm{E}-08$
ND
2. $\mathrm{EE}-04$
ND
ND
$7.0 \mathrm{E}-08$
$9.0 \mathrm{E}-09$
$9.5 \mathrm{E}-07$
$6.4 \mathrm{E}-08$
$1.0 \mathrm{E}-06$
$1.5 \mathrm{E}-04$

$\begin{array}{rc}\text { Concentrations, } \mu \mathrm{Ci} / \mathrm{ml} \\ \text { Average } & \text { Peak } \\ ------ & ------ \\ 1.1 \mathrm{E}-14 & 2.7 \mathrm{E}-14 \\ -1.7 \mathrm{E}-16 & 6.7 \mathrm{E}-16 \\ -1.0 \mathrm{E}-16 & -1.8 \mathrm{E}-17 \\ 5.2 \mathrm{E}-17 & 6.2 \mathrm{E}-16 \\ -2.0 \mathrm{E}-18 & 7.3 \mathrm{E}-17 \\ 2.7 \mathrm{E}-13 & 4.0 \mathrm{E}-13 \\ -6.3 \mathrm{E}-17 & -4.3 \mathrm{E}-17 \\ -4.8 \mathrm{E}-18 & 9.7 \mathrm{E}-17 \\ 8.9 \mathrm{E}-17 & 3.3 \mathrm{E}-16 \\ 1.1 \mathrm{E}-17 & 1.8 \mathrm{E}-17 \\ 1.2 \mathrm{E}-15 & 1.5 \mathrm{E}-15 \\ 8.1 \mathrm{E}-17 & 1.6 \mathrm{E}-16 \\ 1.3 \mathrm{E}-15 & 6.3 \mathrm{E}-15 \\ 1.9 \mathrm{E}-13 & 1.1 \mathrm{E}-12\end{array}$


06:?1:96, 11.21

\begin{tabular}{|c|c|c|}
\hline On/01/ Dates & $\begin{array}{l}2.00 \cdot 13 \\
\text { Total } \alpha \\
\text { if } \mathrm{Cr} / \mathrm{mil}\end{array}$ & $\begin{array}{l}\text { gue } 11 \\
\text { Total } B \\
\Delta \Gamma, i / m\}\end{array}$ \\
\hline$\because 0$ & 15. & 2 SE $13 \pm$ \\
\hline & 1. 9.1 & \\
\hline 0$] / 227 / 95$ & $1.4 E 15 \pm 86 \%$ & \\
\hline$\frac{15}{5}$ & 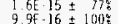 & 6.9E. 14 \\
\hline & $9.9 \mathrm{E} .16 \pm 100 \%$ & \\
\hline & 2. $8 E 15 \pm 45 \%$ & 5.2 \\
\hline & $\begin{array}{l}\frac{1}{9} .9 \mathrm{E} \cdot 15 \pm 699^{\circ} \\
7.3 \mathrm{E}-16 \pm 1204\end{array}$ & \\
\hline & $2.0 E$ & \\
\hline$\frac{5}{5}$ & $3.9 \mathrm{E}$ & \\
\hline 15 & $0 \%$ & 8. OE \\
\hline & 2. $4 E .15 \pm 60 \%$ & $21 \mathrm{E}$ \\
\hline & $\begin{array}{l}.5 E= \pm 16 \pm 0 \% \\
6.4 E \quad 16 \pm 140 \%\end{array}$ & 45 \\
\hline $\begin{array}{l}895 \\
895\end{array}$ & & \\
\hline .05 & & \\
\hline & $\begin{array}{l}3.8 E-15 \\
4 \text { 6E } 16\end{array}$ & $\begin{array}{l}4.7 \mathrm{E} \\
3.9 \mathrm{E}\end{array}$ \\
\hline & $\begin{array}{r}33 \mathrm{~F} \cdot 16 \pm 2502 \\
-8 \cdot 9 \mathrm{E}-17 \pm 5702\end{array}$ & $\begin{array}{l}1.0 E-13 \\
1.7 E \cdot 13\end{array}$ \\
\hline & $99 \mathrm{E}$ & $8.5 E$ \\
\hline & $9.0 \mathrm{E}$ & $5.2 \mathrm{E}$ \\
\hline & 6. $3 \mathrm{E} .15 \pm$ & 倠 \\
\hline & & $\begin{array}{l}8 \mathrm{E} \\
9 \mathrm{E}\end{array}$ \\
\hline & $9.4 E 16$ & $15 \mathrm{E}$ \\
\hline & ${ }_{2}^{4} 2 \mathrm{~F}-16 \pm 3$ & 1. $6 \mathrm{E} .13$ \\
\hline & $\begin{array}{l}2 \\
\frac{2}{7} \\
7\end{array}$ & $4 \mathrm{dE}$ \\
\hline & 3. $6 \mathrm{E} \cdot 15$ & $\begin{array}{l}4.1 \mathrm{t} \\
5.8 \mathrm{E} .\end{array}$ \\
\hline & $\begin{array}{l}9.4 E .16 \\
15 \mathrm{E} .16\end{array}$ & $1.8 \mathrm{E}$ \\
\hline & & \\
\hline $09 / 2$ & $18 E-1$ & $2.1 \mathrm{E}$ \\
\hline $3: 9510 ; 20 ?$ & $\begin{array}{l}1.36 \\
1.36 \\
16\end{array}$ & $\begin{array}{l}\frac{1}{7} .2 \mathrm{E} \\
7.9 \mathrm{E}\end{array}$ \\
\hline & & \\
\hline & & \\
\hline & 10 & \\
\hline & & \\
\hline & 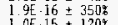 & \\
\hline 5 & & $E-18$ \\
\hline & & \\
\hline & & \\
\hline
\end{tabular}

SYMBOL REY: * Concentration exceeds harning tevel.

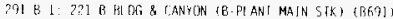

\begin{tabular}{|c|c|c|c|}
\hline $\begin{array}{l}\text { Collect } \\
\text { I inn } \\
\text { Hours }\end{array}$ & $\begin{array}{l}\text { Somple } \\
\text { riowkate } \\
\text { CFM }\end{array}$ & $\begin{array}{c}\text { Sartiple } \\
\text { volune } \\
\mathrm{ft} 3\end{array}$ & $\begin{array}{l}\text { Stack } \\
\text { FlowRate } \\
\text { CFM }\end{array}$ \\
\hline 168.4 & 1.5 & 15000 & 39000 \\
\hline 1680 & & $\begin{array}{l}15000 \\
1 / 1000\end{array}$ & 390000 \\
\hline 170.1 & 1. & 17000 & 39000 \\
\hline 165.0 & & 16000 & 39000 \\
\hline $\begin{array}{l}167.7 \\
1 \in 8.3\end{array}$ & & $\begin{array}{l}17000 \\
16000\end{array}$ & $\begin{array}{l}39900 \\
39000\end{array}$ \\
\hline 168.0 & & $\begin{array}{l}16000 \\
1600\end{array}$ & $\begin{array}{l}3900000 \\
390\end{array}$ \\
\hline 1680 & 1.6 & $\begin{array}{l}16000 \\
1600\end{array}$ & 40000 \\
\hline 1674 & 1. & 17000 & 40000 \\
\hline $\begin{array}{l}69.0 \\
122.7\end{array}$ & 1. & $\begin{array}{l}17000 \\
12000\end{array}$ & $\begin{array}{l}40000 \\
40000\end{array}$ \\
\hline 43.7 & 1. & $\begin{array}{l}4300 \\
4300\end{array}$ & $\begin{array}{l}40000 \\
4\end{array}$ \\
\hline 167.4 & 16 & 16000 & 40000 \\
\hline $\begin{array}{l}169.4 \\
165.0\end{array}$ & & $\begin{array}{l}17000 \\
160000\end{array}$ & $\begin{array}{l}40000 \\
40000\end{array}$ \\
\hline 165.0 & & 16000 & $\begin{array}{l}40000 \\
40000\end{array}$ \\
\hline 168 & & $\begin{array}{l}17000 \\
17000\end{array}$ & $\begin{array}{l}40000 \\
40000\end{array}$ \\
\hline 168.0 & 1.6 & 160000 & $\begin{array}{l}40000 \\
40000\end{array}$ \\
\hline $\begin{array}{l}1684 \\
168.3\end{array}$ & 1. & $\begin{array}{l}16000 \\
17000\end{array}$ & $\begin{array}{l}40000 \\
40000\end{array}$ \\
\hline $\begin{array}{l}100.9 \\
166.7\end{array}$ & $\begin{array}{l}1.0 \\
1.6\end{array}$ & 16000 & 40000 \\
\hline 168.0 & 1.7 & 17000 & 40000 \\
\hline $\begin{array}{l}160.1 \\
163.7\end{array}$ & $\begin{array}{l}1.6 \\
1.6\end{array}$ & $\begin{array}{l}1 / 7800 \\
16000\end{array}$ & $\begin{array}{l}40000 \\
40000\end{array}$ \\
\hline 168.9 & $\begin{array}{l}1.6 \\
1.6\end{array}$ & $\begin{array}{l}16000 \\
16000\end{array}$ & 40000 \\
\hline $\begin{array}{l}168.2 \\
168.2\end{array}$ & 1.6 & $\begin{array}{l}16000 \\
1600\end{array}$ & $\begin{array}{l}400000 \\
4000\end{array}$ \\
\hline 358.0 & $\begin{array}{l}1.6 \\
1.6\end{array}$ & $\begin{array}{l}15000 \\
16000\end{array}$ & 40000 \\
\hline 168.4 & $\begin{array}{l}1.8 \\
1.6\end{array}$ & $\begin{array}{l}16000 \\
1600\end{array}$ & $\begin{array}{l}40000 \\
4000\end{array}$ \\
\hline 168 & 1.6 & 16000 & 40000 \\
\hline 167 & 1.6 & 16030 & 40000 \\
\hline $\begin{array}{l}169 \\
166\end{array}$ & $\begin{array}{l}1.6 \\
1.6\end{array}$ & $\begin{array}{l}16000 \\
15000\end{array}$ & $\begin{array}{l}40000 \\
40000\end{array}$ \\
\hline 168 & 16 & 16000 & 40000 \\
\hline 168. & 1.6 & 16000 & 37000 \\
\hline 166 & $\begin{array}{l}1.6 \\
1.6\end{array}$ & 16000 & 37000 \\
\hline $\begin{array}{l}100 \\
167\end{array}$ & 15 & 15000 & 37000 \\
\hline 169 & 15 & 16000 & 37000 \\
\hline 169 & 1.6 & 16000 & 37000 \\
\hline $\begin{array}{l}165.8 \\
165.9\end{array}$ & $\begin{array}{l}1.6 \\
1.6\end{array}$ & $\begin{array}{l}16000 \\
16000\end{array}$ & 37000 \\
\hline 1689 & 17 & 17000 & 37000 \\
\hline 168.1 & 17 & 18000 & 31000 \\
\hline 169. & 1.7 & 17000 & 37000 \\
\hline $\begin{array}{l}166.2 \\
167.2\end{array}$ & 1.7 & 17000 & 37000 \\
\hline 168.5 & 1 ? & $\begin{array}{l}18000 \\
18000\end{array}$ & $\begin{array}{r}37000 \\
37000\end{array}$ \\
\hline & 17 & 17000 & 37000 \\
\hline 166.9 & 1.7 & 17900 & 37000 \\
\hline $\begin{array}{ll}168.3 \\
0.0\end{array}$ & 0.0 & & $\begin{array}{l}37000 \\
37000\end{array}$ \\
\hline
\end{tabular}

-5 MIA exceeds harning Level. [j]:01:45 to 12/31:95

Parge? 1

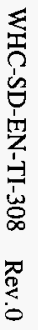

$?$ Reported MDA is negative. 
WHC-SD-EN-TI-308 Rev.0

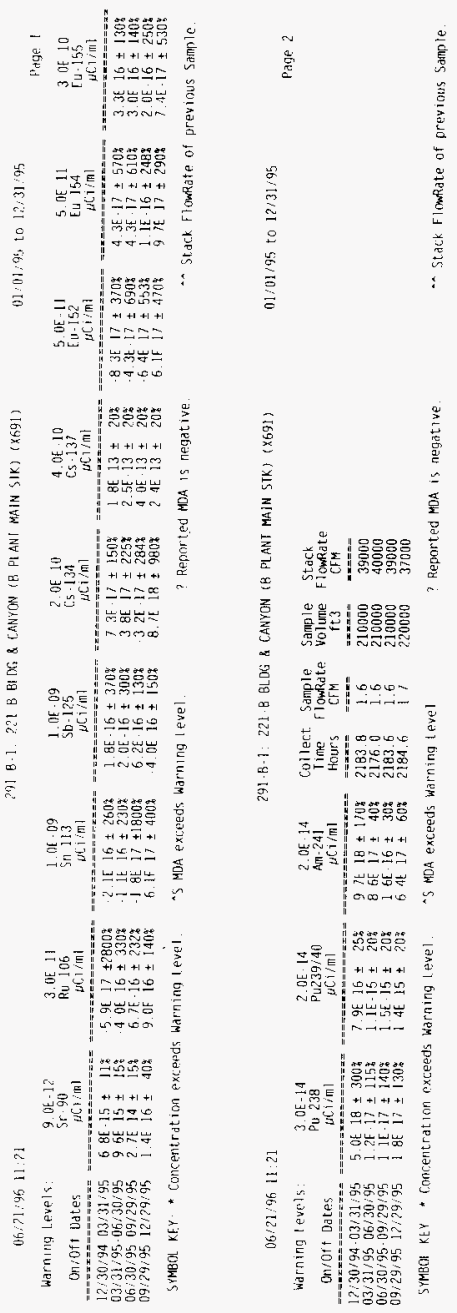


WHC-SD-EN-TI-308 Rev.0

WHC ENVIRONMENTAL RELEASE SUMMARY

\section{6-B-5: 221-BB BUILDING EXHAUST (B686)}

$01 / 01 / 95$ through $12 / 31 / 95$

Total System Flow: $2.2 \mathrm{E}+10$ Liters $=7.9 \mathrm{E}+08$ Cubic Ft

The following factor(s) has (or have) been applied to the radionuclide concentrations used in the calculation of this release to account for sampling filter efficiency, line and bend loss, and humidity. The concentrations vary inversely with the size of the factor(s).

Particulates :

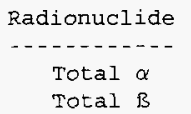

Release, Ci
4. 5E-09
I. $6 \mathrm{E}-08$

0.73

\begin{tabular}{cc} 
Concentrations, $\mu \mathrm{Ci} / \mathrm{ml}$ \\
Average & Peak \\
\hdashline------ \\
1. $5 \mathrm{E}-16$ & $1.8 \mathrm{E}-15$ \\
$5.2 \mathrm{E}-16$ & 5. OE-15
\end{tabular}


(26:21:95 11:21

296 B. 5 22] EB BUII DING FXHNUST (B586

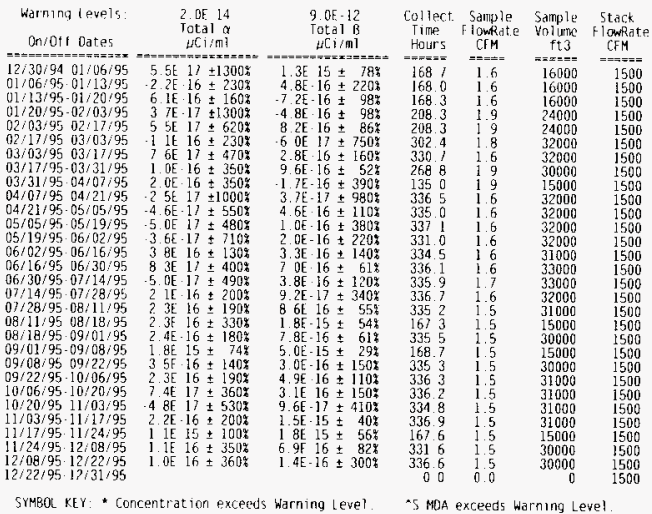

SMMBOL KEY: " Concentration exceeds Warning Level

Mat exceeds Warnng Level.
$01.01 \times 45$ to $12 / 31 / 95$

Paqp 1

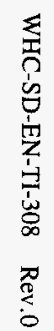


WHC-SD-EN-TI-308 Rev.0

WHC ENVIRONMENTAL RELEASE SUMMARY

\author{
296-B-10: 225-B BUILDING \& CELLS (B748, X748) \\ $01 / 01 / 95$ through $12 / 31 / 95$ \\ Total system Flow: $3.0 E+11$ Liters $=1.0 E+10$ Cubic Ft
}

The following Eactor(s) has (or have) been applied to the radionuclide concentrations used in the calculation of this release to account for sampling filter efficiency, line and bend loss, and humidity. The concentrations vary inversely with the size of the factor(s).

Particulates:

${ }^{90} \mathrm{Sr}$
${ }^{106} \mathrm{Ru}$
${ }^{113} \mathrm{Sn}$
${ }^{125} \mathrm{Sb}$
${ }^{134} \mathrm{Cs}$
${ }^{137} \mathrm{Cs}$
${ }^{152} \mathrm{Eu}$
${ }^{154} \mathrm{Eu}$
${ }^{155} \mathrm{Eu}$
${ }^{238} \mathrm{Pu}$
$239 / 240 \mathrm{Pu}$
$241 \mathrm{Am}$
Total $\alpha$
Total $B$

Release, $\mathrm{Ci}$
$6.0 \mathrm{E}-06$
$2.2 \mathrm{E}-09$
$\mathrm{ND}$
$2.2 \mathrm{E}-08$
$\mathrm{ND}$
$2.9 \mathrm{E}-06$
$1.6 \mathrm{E}-08$
$5.8 \mathrm{E}-08$
$1.1 \mathrm{E}-08$
$\mathrm{ND}$
$5.2 \mathrm{E}-10$
$1.3 \mathrm{E}-08$
$2.0 \mathrm{E}-07$
$1.4 \mathrm{E}-05$

0.73

$\begin{array}{rc}\text { Concentrations, } \mu \mathrm{Ci} / \mathrm{ml} \\ \text { Average } & \text { Peak } \\ -----.--- & -.--14 \\ 1.5 \mathrm{E}-14 & 2.9 \mathrm{E}-14 \\ 5.4 \mathrm{E}-18 & 1.0 \mathrm{E}-15 \\ -2.8 \mathrm{E}-16 & 8.9 \mathrm{E}-17 \\ 5.4 \mathrm{E}-17 & 3.0 \mathrm{E}-16 \\ -1.9 \mathrm{E}-17 & 1.2 \mathrm{E}-16 \\ 7.1 \mathrm{E}-15 & 8.5 \mathrm{E}-15 \\ 3.9 \mathrm{E}-17 & 1.4 \mathrm{E}-16 \\ 1.4 \mathrm{E}-16 & 3.0 \mathrm{E}-16 \\ 2.7 \mathrm{E}-17 & 1.0 \mathrm{E}-16 \\ -1.7 \mathrm{E}-18 & -1.1 \mathrm{E}-18 \\ 1.3 \mathrm{E}-18 & 5.0 \mathrm{E}-18 \\ 3.2 \mathrm{E}-17 & 5.3 \mathrm{E}-17 \\ 4.8 \mathrm{E}-16 & 4.1 \mathrm{E}-15 \\ 3.5 \mathrm{E}-14 & 2.4 \mathrm{E}-13\end{array}$


DE: $31 / 40 \quad 11: 21$

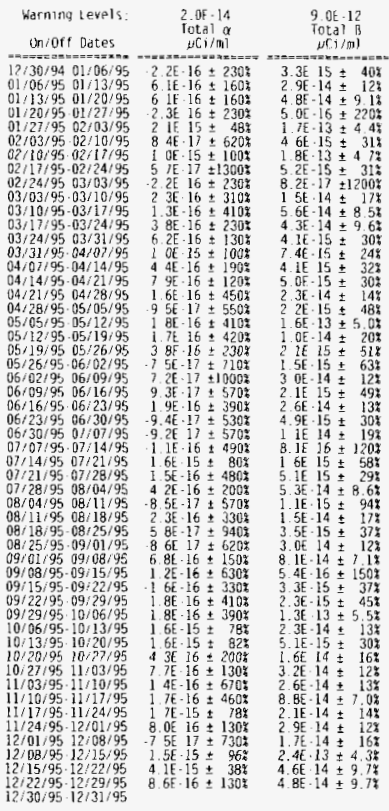

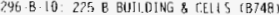

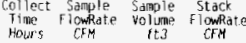

$$
\begin{aligned}
& \begin{array}{cccc}
158.3 & 1.5 & -1600 & -2000 \\
169.3 & 1.6 & 16000 & \\
116.9 & 1.6 & &
\end{array}
\end{aligned}
$$

171.9901 .6

$\begin{array}{ll}1638 & 1 \\ 167.9 & 1 \\ 169 & 1\end{array}$


$06 / 21 / 96,11.21$

296. 8 10: 225 B BUILDINIS \& CFILS $(0748)$

$01 / 01.95$ to $12 / 31 / 95$

\begin{tabular}{|c|c|c|c|c|c|c|c|c|c|}
\hline $\begin{array}{l}\text { Wdming Leveis } \\
\text { Onroff Dates }\end{array}$ & $\begin{array}{l}9 \cdot 0 E \cdot 12 \\
5 \cdot-90 \\
U[1 / \mathrm{m}] \\
===-==\end{array}$ & 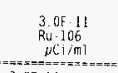 & $\begin{array}{l}1.06 .09 \\
\sin 113 \\
461 / \mathrm{mI} \\
======\end{array}$ & 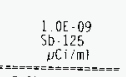 & $\begin{array}{l}2.06 \cdot 10 \\
6.5134 \\
1 C 1 / \mathrm{ml} \\
=====2 .\end{array}$ & $\begin{array}{l}4.0 E-10 \\
\text { Cs } 137 \\
\text { WC } 1 / \pi 1\end{array}$ & $\begin{array}{l}5.0 E-11 \\
\text { Eu } 15 ? \\
u C 1 / m 1 \\
=====x\end{array}$ & 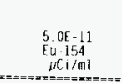 & $\begin{array}{l}\text { Page } 1 \\
\begin{array}{l}3.0 E-10 \\
\text { Eu. } 155 \\
U \mathrm{Ci} / \mathrm{mi}\end{array}\end{array}$ \\
\hline 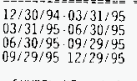 & 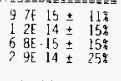 & 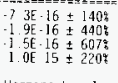 & 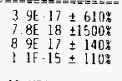 & $\begin{array}{l}2.0 \mathrm{~L} \cdot 17 \pm 1200 \% \\
3.1 \mathrm{IE} 17 \pm 740 \% \\
\text { 1.1E } 16 \pm 237 \% \\
3.0 \mathrm{E} \cdot 16 \pm 130 \%\end{array}$ & 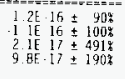 & 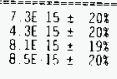 & 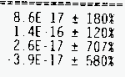 & 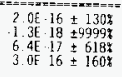 & 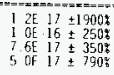 \\
\hline
\end{tabular}

06/21/96, 1121

Warning Levels:

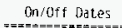

3. 0 . 14

296-B 10: 225 B BUILDING \& CELLS (X748)

$01 / 01 / 95$ to $12 / 31 / 95$

Page 2

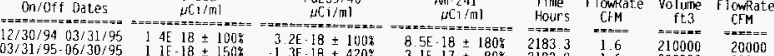

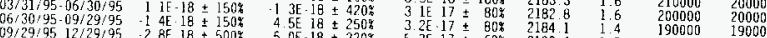

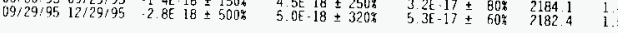

SMBQQ KEY: * Concentration exceeds warning level. IS MOA exceeds Warning level

190000
? Reported MDA is negative. 


\title{
WHC-SD-EN-TI-308 Rev.0
}

WHC ENVIRONMENTAL RELEASE SUMMARY

\author{
296-B-13: 221-BF BCP TANKS \& CONDENSATE (B690) \\ $01 / 01 / 95$ through $12 / 31 / 95$ \\ Total system Flow: 1. OE+10 Liters $=3.6 \mathrm{E}+08$ Cubic Ft
}

The following factor(s) has (or have) been applied to the radionuclide concentrations used in the calculation of this release to account for sampling filter efficiency, line and bend loss, and humidity. The concentrations vary inversely with the size of the factor $(s)$.

$\begin{array}{cccc}\text { Particulates: } & 0.73 \\ \text { Radionuclide } & \text { Release, Ci } & \begin{array}{c}\text { Concentrations, } \mu \mathrm{Ci} / \mathrm{ml} \\ \text { Average }\end{array} \\ \text { Peak } \\ \text { Total } \alpha & 2.8 \mathrm{E}-09 & 2.0 \mathrm{E}-16 & 1.9 \mathrm{E}-15 \\ \text { Total } B & 1.5 \mathrm{E}-08 & 1.0 \mathrm{E}-15 & 3.2 \mathrm{E}-15\end{array}$




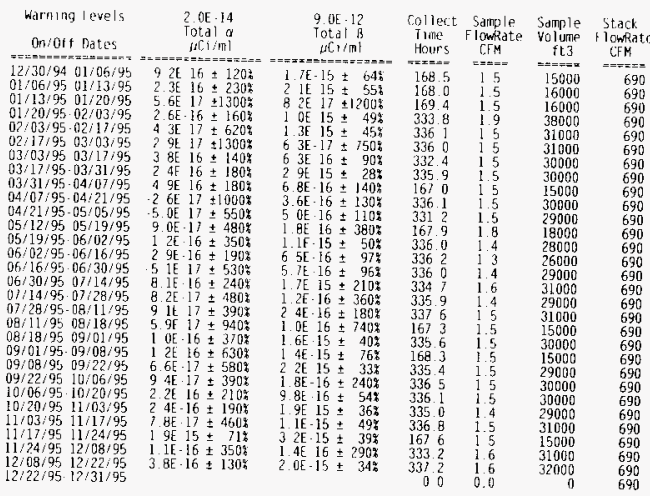

SYMBOL KEY: * Concentration exceeds warning Level

is MDA exceeds Warning Level 
WHC-SD-EN-TI-308 Rev.0

This page intentionally left blank 
WHC-SD-EN-TI-308 Rev.0

2.4.3 East Tank Farms 
WHC-SD-EN-TI-308 Rev.0

WHC ENVIRONMENTAL RELEASE SUMMARY

$06 / 15 / 96$

296-A-17\& 296-P-26: 241-AY, AZ TANK EXHAUSTERS (E059, E039)

$01 / 01 / 95$ through $12 / 31 / 95$

Total System Flow: $9.2 \mathrm{E}+10$ Liters $=3.3 \mathrm{E}+09$ Cubic $\mathrm{Ft}$

${ }^{90} \mathrm{Sr}$
${ }^{106} \mathrm{Ru}$
${ }^{113} \mathrm{Sn}$
${ }^{125} \mathrm{Sb}$
${ }^{129} \mathrm{I}$
${ }^{134} \mathrm{Cs}$
${ }^{137} \mathrm{Cs}$
${ }^{152} \mathrm{Eu}$
${ }^{154} \mathrm{Eu}$
${ }^{155} \mathrm{Eu}$
${ }^{238} \mathrm{Pu}$
$239 / 240 \mathrm{Pu}$
${ }^{241} \mathrm{Am}$
Total $\alpha$
Total $\beta$

$\begin{array}{cc}\text { Halflife } & \text { Release, Ci } \\ 29.12 \text { Years } & 5.4 \mathrm{E}-06 \\ 368 \text { Days } & 4.0 \mathrm{E}-06 \\ 115 \text { Days } & 5.3 \mathrm{E}-07 \\ 2.8 \text { Years } & 9.5 \mathrm{E}-07 \\ 1.57 \mathrm{E}+7 \text { Years } & 2.7 \mathrm{E}-04 \\ 2.1 \text { Years } & 1.8 \mathrm{E}-08 \\ 30 \text { Years } & 6.2 \mathrm{E}-05 \\ 13.3 \text { Years } & \mathrm{ND} \\ 8.8 \text { Years } & 8.8 \mathrm{E}-09 \\ 4.96 \text { Years } & 3.1 \mathrm{E}-08 \\ 87.8 \text { Years } & 4.6 \mathrm{E}-09 \\ 24,131 \text { Years* } & 1.4 \mathrm{E}-08 \\ 432 \text { Years } & 1.2 \mathrm{E}-08 \\ \text { n/a } & 2.2 \mathrm{E}-07 \\ \text { n/a } & 3.6 \mathrm{E}-05\end{array}$

- The halflife shown for Total Uranium is the halflife of ${ }^{238} \mathrm{U}$. The halflife shown for mixed ${ }^{239 / 240} \mathrm{Pu}$ is the halflife of ${ }^{239} \mathrm{Pu}$. 
$06: 21 / 9611 \cdot 21$

\begin{tabular}{|c|c|c|c|c|c|c|}
\hline $\begin{array}{l}\text { Wdining lovels } \\
\text { Onioff Dates } \\
==-=z===-2\end{array}$ & 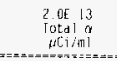 & 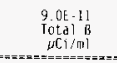 & $\begin{array}{l}\text { Collect } \\
\text { Time } \\
\text { Hours } \\
=-===\end{array}$ & $\begin{array}{c}\text { Sample } \\
+ \text { lomkatc } \\
\text { CFM } \\
== \pm=\end{array}$ & $\begin{array}{c}\text { Sample } \\
\text { volune } \\
\text { ft3 } \\
==-2\end{array}$ & $\begin{array}{c}\text { Stack } \\
\text { F iowRa } \\
C F M\end{array}$ \\
\hline 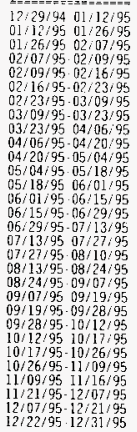 & 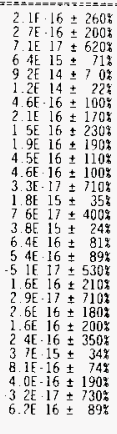 & 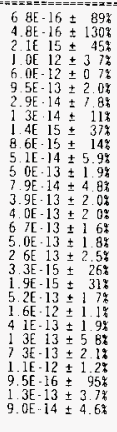 & $\begin{array}{r}-332 . \\
332.0 \\
335.0 \\
788.7 \\
48.2 \\
177.0 \\
155.7 \\
3369 \\
334.4 \\
335.8 \\
337.0 \\
335.8 \\
335.5 \\
336.1 \\
335.1 \\
337.5 \\
334.9 \\
336.0 \\
336.1 \\
253.8 \\
340.6 \\
279.0 \\
2205 \\
340.0 \\
108.0 \\
222.9 \\
337.1 \\
171.5 \\
382.9 \\
343.8 \\
0.0\end{array}$ & $\begin{aligned}==2= \\
1.3 \\
1.3 \\
1.1 \\
1.6 \\
1.8 \\
1.7 \\
1.8 \\
1.9 \\
1.9 \\
1.8 \\
1.8 \\
1.7 \\
1.9 \\
1.8 \\
1.9 \\
1.9 \\
1.9\end{aligned}$ & $\begin{array}{r}==r-2 \\
25000 \\
26000 \\
180000 \\
4600 \\
19000 \\
16000 \\
36000 \\
37000 \\
39000 \\
38000 \\
35000 \\
37000 \\
35000 \\
39000 \\
37000 \\
38000 \\
37000 \\
38000 \\
30000 \\
40000 \\
41000 \\
30000 \\
41000 \\
14000 \\
20000 \\
39000 \\
19000 \\
35000 \\
32000 \\
0\end{array}$ & $\begin{array}{l}380 \\
380 \\
380 \\
3800 \\
3300 \\
380 \\
380 \\
3900 \\
5900 \\
5900\end{array}$ \\
\hline
\end{tabular}

SYMEd KFy: "Concentration exceeds hidning Level.

^5 MDA exceeds Warming Level 
0म:?1/46 11.?

996 A.17: 241 AY AL TANS IE026,

UL. 01195 to $12: 37195$

Pag̣e !

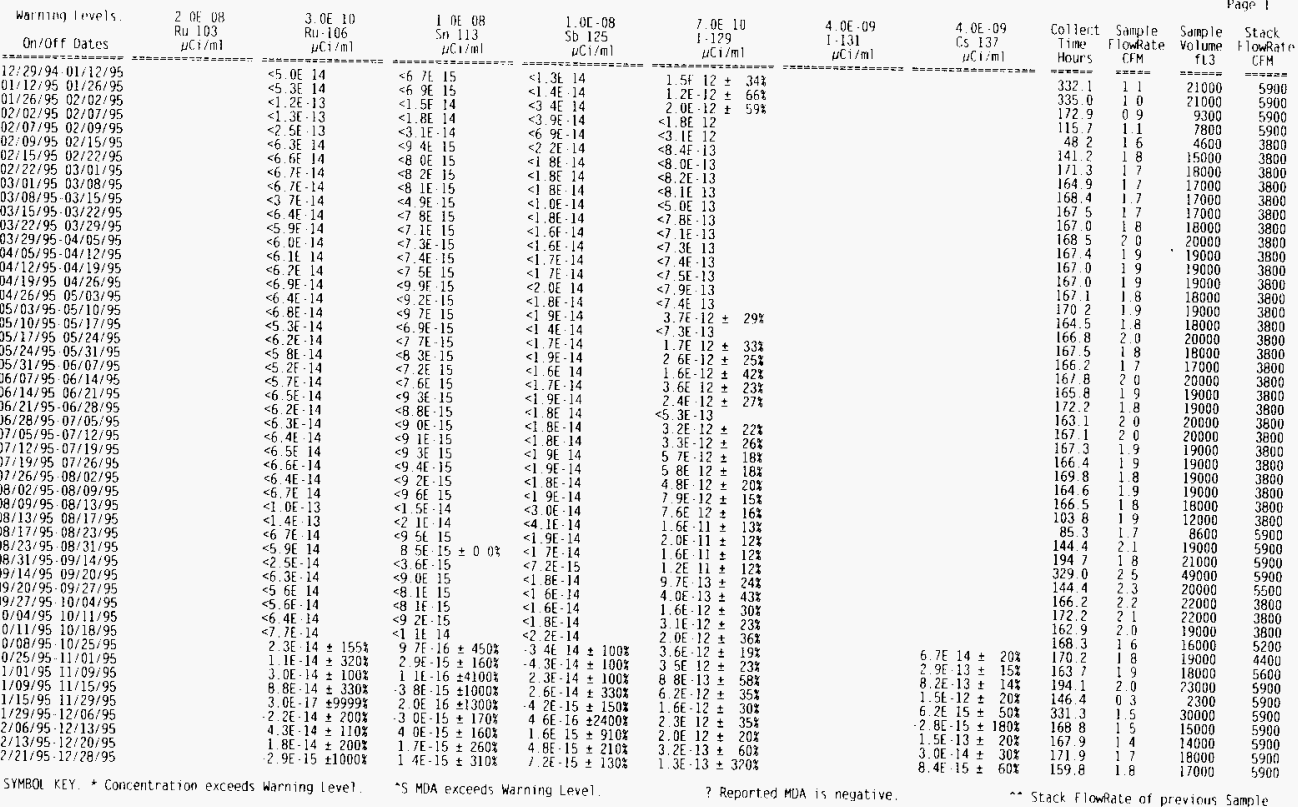




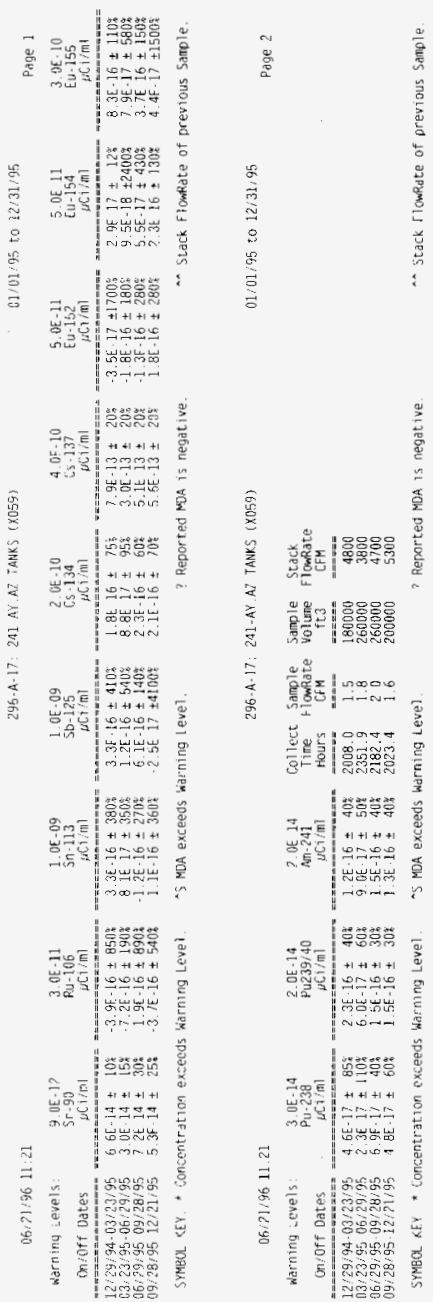




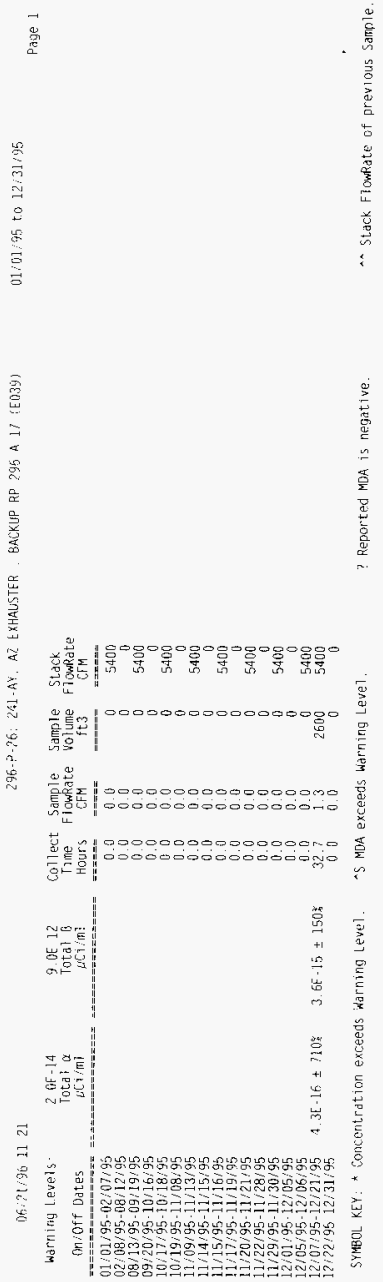


WHC-SD-EN-TI-308 Rev.0

WHC ENVIRONMENTAL RELEASE SUMMARY

$06 / 15 / 96$

296-A-20: ALL AZ ANNULI (E197)

$01 / 01 / 95$ through $12 / 31 / 95$

Total System Flow: $2.6 \mathrm{E}+10$ Liters $=5.7 \mathrm{E}+08$ Cubic Ft

The following factor(s) has (or have) been applied to the radionuclide concentrations used in the calculation of this release to account for sampling filter efficiency, line and bend loss, and humidity. The concentrations vary inversely with the size of the factor(s).

\begin{tabular}{cccc}
\multicolumn{2}{c}{ Particulates: } & 0.84 \\
Radionuclide & Release, Ci & Concentrations, $\mu \mathrm{Ci} / \mathrm{ml}$ \\
Total $\alpha$ & $1.1 \mathrm{E}-08$ & $-15 \mathrm{E}-16$ & $3.1 \mathrm{E}-15$ \\
Total $B$ & $3.1 \mathrm{E}-08$ & $1.6 \mathrm{E}-15$ & $6.0 \mathrm{E}-15$
\end{tabular}


06:21:96 11:2

\begin{tabular}{|c|c|c|c|c|c|c|}
\hline $\begin{array}{l}\text { Warning Levels: } \\
\text { Onioff Dotes }\end{array}$ & $\begin{array}{c}2 \text { OE } 14 \\
\text { Total } \alpha \\
\text { uf. } 1: \mathrm{m}]\end{array}$ & $\begin{array}{l}9.0 E-12 \\
\text { Totdi } 13 \\
\text { HCi } 1 ; m 1\end{array}$ & $\begin{array}{l}\text { Co?lect } \\
\text { Thine } \\
\text { Hours }\end{array}$ & $\begin{array}{l}\text { Somple } \\
\text { Flowkat te } \\
\text { CFM }\end{array}$ & $\begin{array}{c}\text { Sample } \\
\text { volume } \\
\text { ft3 }\end{array}$ & $\begin{array}{c}\text { Stack } \\
\text { FlowRate } \\
\text { GFM }\end{array}$ \\
\hline 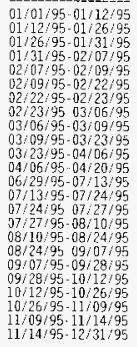 & 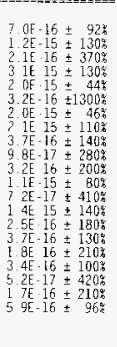 & 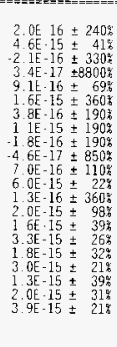 & $\begin{array}{r}x==== \\
0.07 \\
335.0 \\
129.5 \\
160.4 \\
43.7 \\
300.5 \\
32.2 \\
256.1 \\
80.7 \\
334.8 \\
335.8 \\
337.0 \\
335.3 \\
256.3 \\
79.7 \\
336.0 \\
335.9 \\
340.8 \\
499 \\
336.9 \\
33.6 \\
337.2 \\
0.0 \\
0.0\end{array}$ & $\begin{array}{l}0.0 \\
1.4 \\
1.3 \\
1.5 \\
1.5 \\
1.3 \\
1.4 \\
1.4 \\
1.5 \\
1.5 \\
1.5 \\
1.0 \\
1.2 \\
1.8 \\
1.7 \\
1.5 \\
1.6 \\
1.8 \\
1.5 \\
1.9 \\
1.8 \\
0.0 \\
0.0\end{array}$ & $\begin{array}{r}29000 \\
29000 \\
9900 \\
15000 \\
3800 \\
23000 \\
2800 \\
21000 \\
7300 \\
30000 \\
29000 \\
21000 \\
23000 \\
27000 \\
7900 \\
30000 \\
32000 \\
37000 \\
49000 \\
35000 \\
37000 \\
37000 \\
0 \\
0\end{array}$ & $\begin{array}{l}1600 \\
1600 \\
1600 \\
1600 \\
1600 \\
1100 \\
1600 \\
1600 \\
1600 \\
1600 \\
1600 \\
1100 \\
220 \\
150 \\
1600 \\
1600 \\
1600 \\
1600 \\
1600 \\
1600 \\
1600 \\
1600 \\
1600 \\
0\end{array}$ \\
\hline
\end{tabular}

5YMBOL. KEY: * Concentration. exceeds wärning Level.
295-A.20-ALL A? ANKULI IE197\}

$$
\begin{aligned}
& \text { tack } \\
& \text { OwRate } \\
& 6-M \\
& 1600 \\
& 1600 \\
& 1600 \\
& 1600 \\
& 1600 \\
& 1600 \\
& 1100 \\
& 1600 \\
& 1600 \\
& 1600 \\
& 1600 \\
& 1600 \\
& 1100 \\
& 220 \\
& 150 \\
& 1600 \\
& 1600 \\
& 1600 \\
& 1600 \\
& 1600 \\
& 1600 \\
& 1600 \\
& 1600 \\
& 1600 \\
& 0
\end{aligned}
$$

^5 MDA exceeds warning Level
(11:01:95 to 12:31:95

$\left.p_{\text {dge }}\right]$
? Reported MDA is negative. 
WHC-SD-EN-TI-308 Rev.0

WHC ENVIRONMENTAL RELEASE SUMMARY

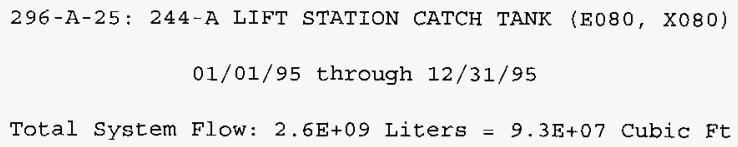

The following factor(s) has (or have) been applied to the radionuclide concentrations used in the calculation of this release to account for sampling filter efficiency, line and bend loss, and humidity. The concentrations vary inversely with the size of the factor (s).

Particulates:

Radionuclide
${ }^{90} \mathrm{Sr}$
${ }^{106} \mathrm{Ru}$
${ }^{113} \mathrm{Sr}$
${ }^{125} \mathrm{Sb}$
${ }^{134} \mathrm{Cs}$
${ }^{137} \mathrm{Cs}$
${ }^{152} \mathrm{Eu}$
${ }^{154} \mathrm{Eu}$
${ }^{155} \mathrm{Eu}$
${ }^{238} \mathrm{Pu}$
${ }^{239 / 240} \mathrm{Pu}$
${ }^{241} \mathrm{Am}$
Total $\alpha$
Total B

Release, Ci
3.2E-08
ND
ND
4. OE-10
4. OE-11
1. $4 \mathrm{E}-06$
ND

1. $4 E-10$ ND

5. $7 \mathrm{E}-11$

1. $7 \Xi-10$

4. $1 \mathrm{E}-10$

5. $6 \mathrm{E}-09$

1. $2 E-06$
0.81

\begin{tabular}{|c|c|}
\hline $\begin{array}{c}\text { Concentrati } \\
\text { Average }\end{array}$ & $\begin{array}{c}\text { ons, } \mu \mathrm{Ci} / \mathrm{ml} \\
\text { Peak }\end{array}$ \\
\hline$-\cdots----$ & ------ \\
\hline $9.9 E-15$ & $2.3 E-14$ \\
\hline$-2.1 E-16$ & $6.2 \mathrm{E}-16$ \\
\hline$-2.0 E-16$ & $-4.8 E-17$ \\
\hline 1. $2 \mathrm{E}-16$ & 1. $7 \mathrm{E}-16$ \\
\hline 1. $2 \mathrm{E}-17$ & $5.4 E-17$ \\
\hline $4.2 \mathrm{E}-13$ & 5. $2 E-13$ \\
\hline$-7.4 \mathrm{E}-17$ & $9.1 \mathrm{E}-17$ \\
\hline $4.3 \mathrm{E}-17$ & 1. $8 \mathrm{E}-16$ \\
\hline$-4.4 E-17$ & $4.2 E-16$ \\
\hline $1.8 \mathrm{E}-17$ & $2.2 \mathrm{E}-17$ \\
\hline $5.1 \mathrm{E}-17$ & $7.5 E-17$ \\
\hline $1.3 E-16$ & $3 \cdot 1 E-16$ \\
\hline 1. $7 \mathrm{E}-15$ & $1.1 \mathrm{E}-14$ \\
\hline $3.9 E-13$ & 1. $1 E-12$ \\
\hline
\end{tabular}


WHC-SD-EN-TI-308 Rev.0
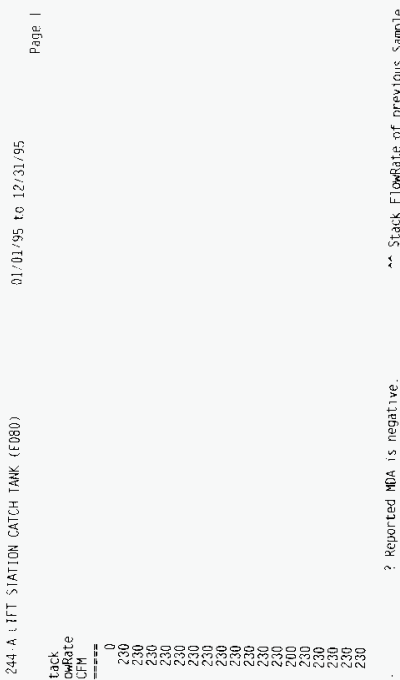
स्थ

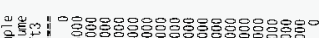

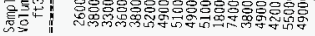

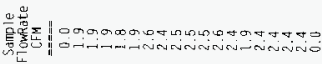

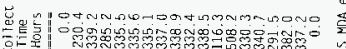

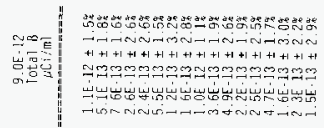

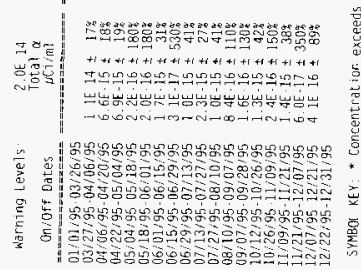


WHC-SD-EN-TI-308 Rev.0

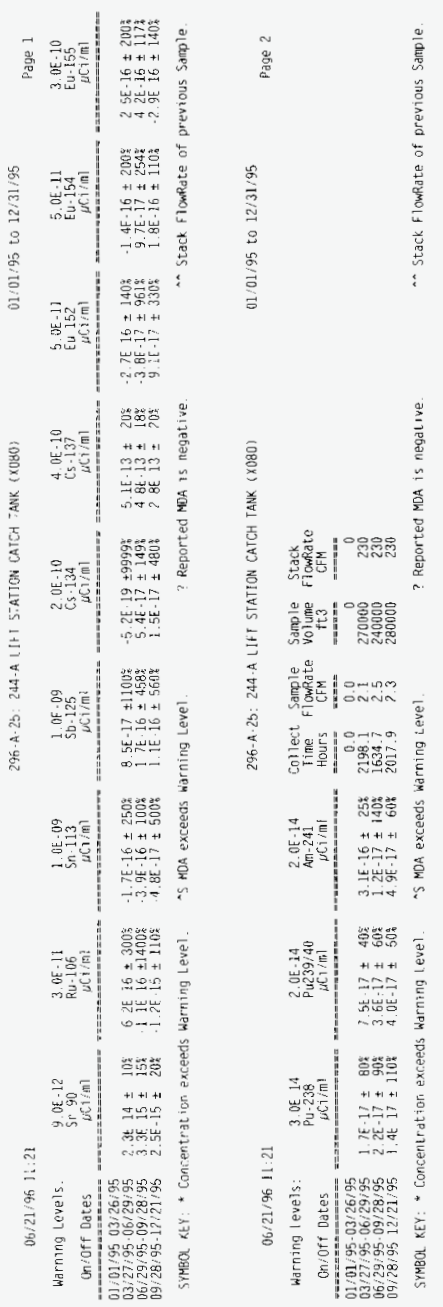


WHC-SD-EN-TI-308 Rev.0

WHC ENVIRONMENTAL RELEASE SUMMARY

296-A-26: 204-AR WASTE UNLOADING FACILITY (E297)

$01 / 01 / 95$ through $12 / 31 / 95$

Total System Flow: 2.9E+10 Liters $=1.0 E+09$ Cubic Ft

The following factor(s) has (or have) been applied to the radionuclide concentrations used in the calculation of this release to account for sampling filter efficiency, line and bend loss, and humidity. The concentrations vary inversely with the size of the factor $(s)$.

Particulates:

Radionuclide ............

Total $\alpha$

Total $B$

Release, $\mathrm{Ci}$
7.2E-09
2. $4 \mathrm{E}-08$

Release, $\mathrm{Ci}$

2. $4 \mathrm{E}-08$
0.73

$\begin{array}{cc}\begin{array}{c}\text { Concentrations, } \mu \mathrm{Ci} / \mathrm{ml} \\ \text { Average }\end{array} & \text { Peak } \\ ----- & ----- \\ 1.8 \mathrm{E}-16 & 1.2 \mathrm{E}-15 \\ \text { 6.0E-16 } & \text { 3. } 6 \mathrm{E}-15\end{array}$


WHC-SD-EN-TI-308 Rev.0

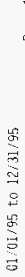

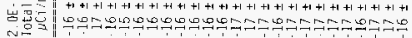

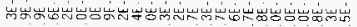

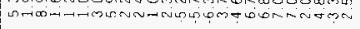

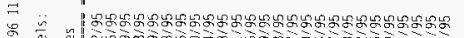

S

告

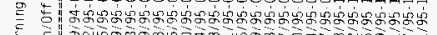

₹ 1 


\section{WHC-SD-EN-TI-308}

Rev. 0

WHC ENVIRONMENTAL RELEASE SUMMARY

296-A-27: ALL 241-AW TANKS' EXHAUSTER (E270, E933)

$$
01 / 01 / 95 \text { through } 12 / 31 / 95
$$

Total System Flow: $1.8 \mathrm{E}+10$ Liters $=6.5 \mathrm{E}+08$ Cubic Ft

Radionuclide

${ }^{90} \mathrm{Sr}$

${ }^{106} \mathrm{Ru}$

${ }^{113} \mathrm{Sn}$

$125 \mathrm{sb}$

${ }^{129} \mathrm{I}$

${ }^{134} \mathrm{Cs}$

${ }^{137} \mathrm{Cs}$

${ }^{152} \mathrm{Eu}$

${ }^{154} \mathrm{Ev}$

${ }^{155} \mathrm{Eu}$

${ }^{238} \mathrm{Pu}$

239/240 $\mathrm{Pu}$

${ }^{241} \mathrm{Am}$

Total $\alpha$

Total B

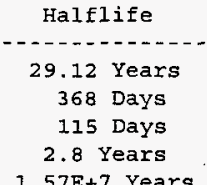

I. $57 \mathrm{E}+7$ Years

2.1 Years

30 Years

13.3 Years

8.8 Years

4.96 Years

87.8 Years

24,131 Years*

432 Years

$\mathrm{n} / \mathrm{a}$

$\mathrm{n} / \mathrm{a}$
Release, Ci

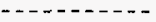

ND

1. $3 \mathrm{E}-06$

1. $2 \mathrm{E}-07$

3. 5E-07

1. 4 E- 05 ND

2. 5E-09

ND

$\mathrm{ND}$

ND

ND

1. $7 \mathrm{E}-10$

8. $2 \mathrm{E}-10$

7. 9 E- 09

1. $9 \mathrm{E}-06$

* The halflife shown for Total Uranium is the halflife of ${ }^{238} \mathrm{U}$. The halflife shown for mixed ${ }^{239 / 240} \mathrm{Pu}$ is the halflife of ${ }^{239} \mathrm{Pu}$. 
WHC-SD-EN-TI-308 Rev.0



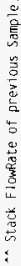

焉

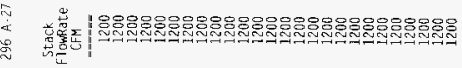

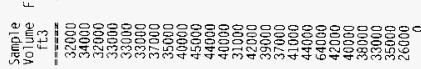
辛

总妾 范叫

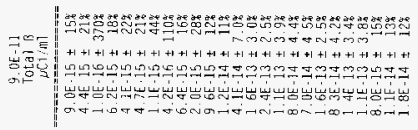

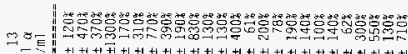

U. $1+1+1+1+1+1+1+1+1+1+1+1+1+1+1+1+1+1+1+1+1+1+1+1+1+1$

岁它

- | |

in

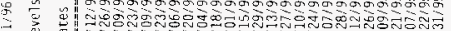

य

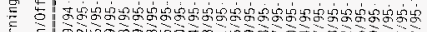

है

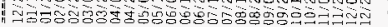




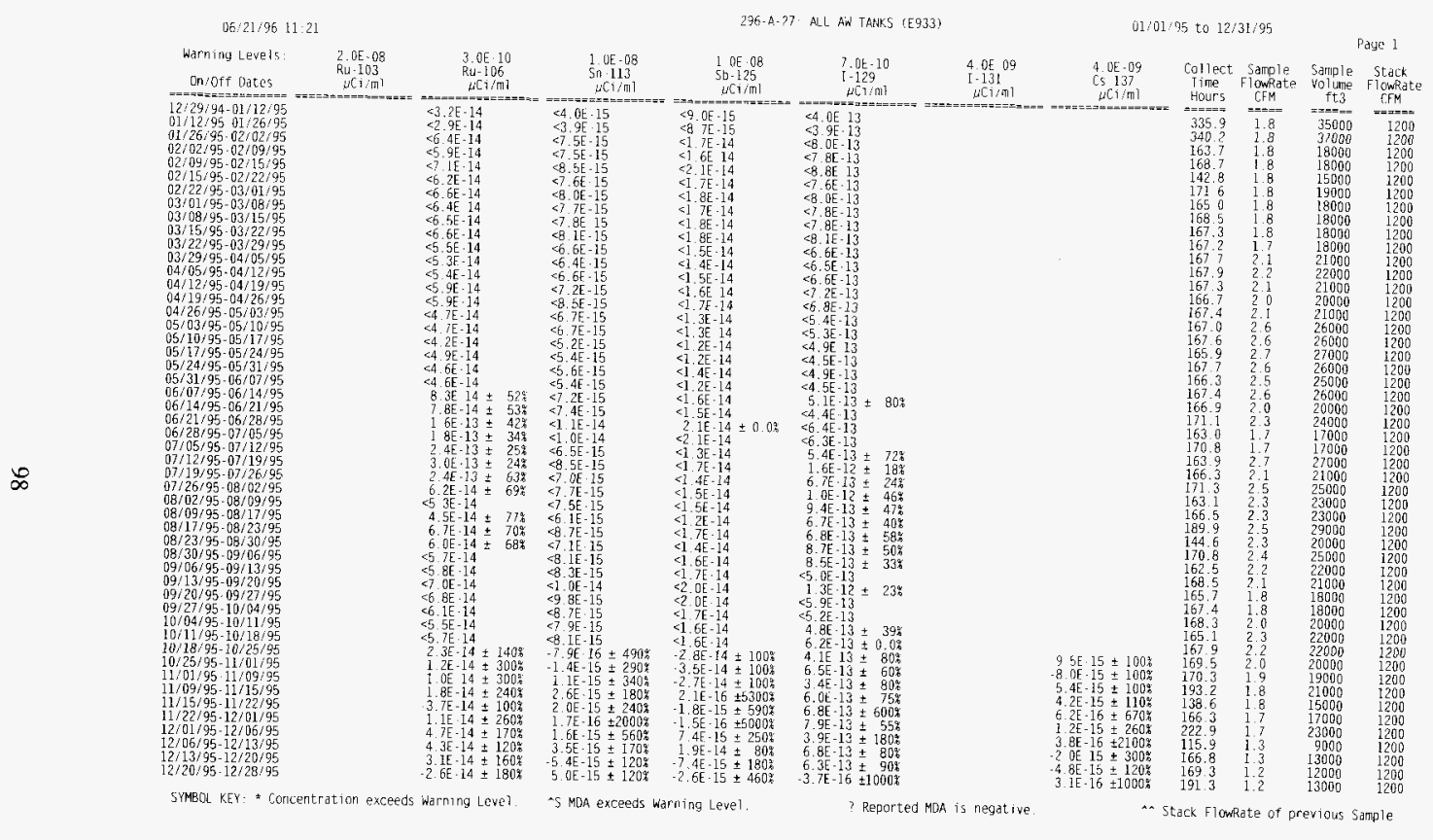


$06 / 21 / 9611.21$

796 A 27: ALL AW TANKS (X?20)

\begin{tabular}{|c|c|c|c|c|c|c|c|c|c|}
\hline $\begin{array}{l}\text { Warling levels. } \\
\text { On/oft Dates }\end{array}$ & 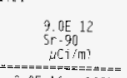 & 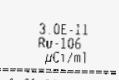 & 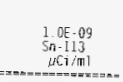 & 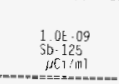 & $\begin{array}{r}2.0 E-10 \\
65-134 \\
4 C i / \pi 1 \\
0.0\end{array}$ & $\begin{array}{l}4.0610 \\
0.137 \\
0 \% 1 / \mathrm{m1} \\
\end{array}$ & 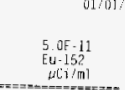 & 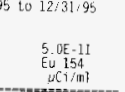 & 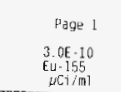 \\
\hline 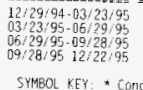 & 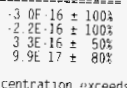 & $\begin{array}{l}1.3 \mathrm{E}-15 \pm 99 . \\
4.1 \mathrm{E}-16 \pm 1500 \\
7.8 \mathrm{E}-16 \pm 111 \mathrm{~h} \\
1.2 \mathrm{E}-15 \pm 90 \%\end{array}$ & 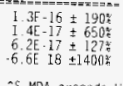 & 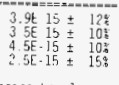 & $\begin{array}{r}1.6 \mathrm{E}-17 \pm 690 \% \\
1.2 \mathrm{E} \cdot 17 \pm 570 \% \\
1.1 \mathrm{E} \cdot 17 \pm 653 \% \\
-2.6 \mathrm{E} \cdot 17 \pm 33 \% \%\end{array}$ & $\begin{array}{l}-59 \mathrm{~g} 17 \pm 1500^{\circ} \\
1.1 \mathrm{~F}-16 \pm 50^{\circ} \\
6.5 \mathrm{E} \cdot 17 \pm 104 \% \\
1.5 \mathrm{E}-16 \pm 60^{\circ}\end{array}$ & 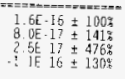 & 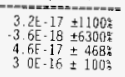 & $\begin{array}{r}1.7 E \cdot 16 \pm 1308 \\
1.80-15 \pm 1004 \\
4.2 E-17 \pm 3928 \\
1.3 E 16 \pm 170\end{array}$ \\
\hline
\end{tabular}

$06 / 21 / 96 \quad 11.21$

296-A.27: ALL AW TANKS ( $\times 270)$

b1/01/95 to $12 / 31 / 95$

Page 2

\begin{tabular}{|c|c|c|c|c|c|c|c|}
\hline $\begin{array}{l}\text { Warnirg Levels: } \\
\text { In!Uff Dates }\end{array}$ & 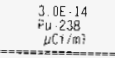 & 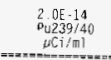 & 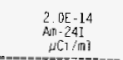 & $\begin{array}{l}\text { Sol lect } \\
\text { Time } \\
\text { Hours }\end{array}$ & $\begin{array}{l}\text { Sample } \\
\text { Flonfate } \\
\text { CFM }\end{array}$ & $\begin{array}{l}\text { Satple } \\
\text { yolume } \\
\text { ft3 }\end{array}$ & $\begin{array}{l}\text { Stack } \\
\text { Flowhate } \\
\text { CFM }\end{array}$ \\
\hline 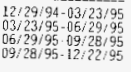 & 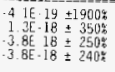 & $\begin{array}{l}3.6 \mathrm{E}-18 \pm 100 \% \\
6 \mathrm{GE}-19 \pm 190 \% \\
3.2 \mathrm{E}-18 \pm 300 \% \\
1.8 \mathrm{E}-17 \pm 120 \%\end{array}$ & $\begin{array}{l}2.2 \mathrm{E}-17 \pm 800 \\
3.2 \mathrm{E}-17 \pm 260 \\
3.6 \mathrm{E}-17 \pm 902\end{array}$ & $\begin{array}{l}=== \pm= \\
2015.9 \\
2352.6 \\
2184.5 \\
2033.3\end{array}$ & $\begin{array}{r}1.6 \\
1.9 \\
2.0 \\
1.8\end{array}$ & $\begin{array}{l}200000 \\
270000 \\
270000 \\
210000\end{array}$ & $\begin{array}{l}1200 \\
1200 \\
1200\end{array}$ \\
\hline
\end{tabular}

SYMBGI KEY: * Concentratian exceeds warming Level. Is MaA exceeds Warning Level. 
WHC-SD-EN-TI-308 Rev.0

$06 / 14 / 96$

WHC ENVIRONMENTAL RELEASE SUMMARY

296-A-28: ALL AW ANNULI (E272)

$01 / 01 / 95$ through $12 / 31 / 95$

Total System Flow: $9.3 \mathrm{E}+10$ Liters $=3.3 \mathrm{E}+09$ Cubic Ft

The following factor(s) has (or have) been applied to the radionuclide concentrations used in the calculation of this release to account for sampling filter efficiency, line and bend loss, and humidity. The concentrations vary inversely with the size of the factor (s).

Particulates:

Radionuclide Total $\alpha$

Total $B$
Release, Ci
4. $6 E-08$
3. $9 E-07$

0.47

\begin{tabular}{cc} 
Concentrations, $\mu \mathrm{Ci} / \mathrm{ml}$ \\
Average & Peak \\
\hdashline$-\cdots-$ & ---- \\
$2.3 \mathrm{E}-16$ & $6.0 \mathrm{E}-16$ \\
$2.0 \mathrm{E}-15$ & $6.9 \mathrm{E}-15$
\end{tabular}


$06 / 21 / 96 \quad 11: 21$

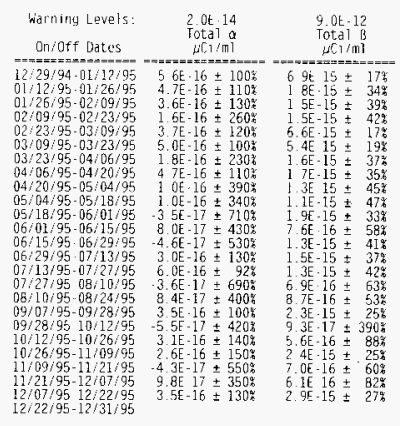

5MMBOL KEY: * Concentration exceeds warning Level
296 A.28. ALL AW ANNLLI (F27)?

\begin{tabular}{|c|c|c|c|}
\hline $\begin{array}{l}\text { Collect } \\
\text { Time } \\
\text { Heurs }\end{array}$ & $\begin{array}{c}\text { Sample } \\
\text { rouprate } \\
\text { CFM }\end{array}$ & $\begin{array}{c}\text { Sample } \\
\text { yolume } \\
f \neq 3\end{array}$ & $\begin{array}{l}\text { Stack } \\
\text { Flowrite } \\
\text { Crint }\end{array}$ \\
\hline $\begin{array}{r}335.6 \\
340.2 \\
332.4 \\
335.1 \\
3355 \\
337.0 \\
335.5 \\
337.1 \\
3399 \\
331.5 \\
336.4 \\
336.5 \\
336.6 \\
335.4 \\
333.6 \\
336.5 \\
336.3 \\
509.0 \\
332.8 \\
335.3 \\
340.3 \\
292.5 \\
376.4 \\
356.3 \\
0.0\end{array}$ & $\begin{array}{l}1.6 \\
1.6 \\
1.6 \\
1.7 \\
1.6 \\
1.6 \\
1.6 \\
1.7 \\
1.4 \\
1.7 \\
1.7 \\
1.7 \\
1.6 \\
1.9 \\
1.6 \\
1.7 \\
1.6 \\
1.7 \\
1.7 \\
2.2 \\
1.9 \\
1.5 \\
1.5 \\
0.0\end{array}$ & $\begin{array}{r}33000 \\
33000 \\
33000 \\
34000 \\
33000 \\
33000 \\
33000 \\
34000 \\
280000 \\
33000 \\
33000 \\
34000 \\
32000 \\
380000 \\
33000 \\
33000 \\
34000 \\
48000 \\
34000 \\
34000 \\
45000 \\
33000 \\
34000 \\
33000 \\
0\end{array}$ & $\begin{array}{l}6200 \\
6200 \\
6200 \\
6200 \\
62200 \\
6200 \\
6200 \\
6200 \\
6200 \\
6200 \\
6200 \\
6200 \\
6200 \\
6200 \\
6200 \\
6200 \\
6200 \\
6200 \\
6200 \\
6200 \\
6200 \\
6200 \\
6200 \\
6200 \\
6200\end{array}$ \\
\hline
\end{tabular}

B1701:95 to 12:31/95

Päge 1

as MDA exceeds Warming Level. 


\title{
WHC-SD-EN-TI-308 Rev.0
}

$06 / 14 / 96$

WHC ENVIRONMENTAI, RELEASE SUMMARY

\author{
296-A-29: ALL AN TANKS (E901, X901) \\ $01 / 01 / 95$ through $12 / 31 / 95$ \\ Total System Flow: $1.4 \mathrm{E}+10$ Liters $=4.9 \mathrm{E}+08$ Cubic Ft
}

The following factor(s) has (or have) been applied to the radionuclide concentrations used in the calculation of this release to account for sampling filter efficiency, line and bend loss, and humidity. The concentrations vary inversely with the size of the factor (s).

$$
\text { Particulates: } \quad 0.53
$$

${ }^{90} \mathrm{Sr}$
${ }^{106} \mathrm{Ru}$
${ }^{113} \mathrm{Sn}$
${ }^{125} \mathrm{Sb}$
${ }^{134} \mathrm{Cs}$
${ }^{137} \mathrm{Cs}$
${ }^{152} \mathrm{Eu}$
${ }^{154} \mathrm{Eu}$
${ }^{155} \mathrm{Eu}$
${ }^{238} \mathrm{Pu}$
$239 / 240 \mathrm{Pu}$
$241 \mathrm{Am}$
Total $\alpha$
Total $B$

Total $B$

Release, Ci
$2.0 \mathrm{E}-08$
$\mathrm{ND}$
$\mathrm{ND}$
$\mathrm{ND}$
$\mathrm{ND}$
$1.9 \mathrm{E}-10$
$\mathrm{ND}$
$9.4 \mathrm{E}-10$
$\mathrm{ND}$
$3.0 \mathrm{E}-11$
$1.1 \mathrm{E}-10$
$1.1 \mathrm{E}-09$
8. $0 \mathrm{E}-09$
$7.9 \mathrm{E}-07$

$\begin{array}{cc}\text { Concentrations, } \mu \mathrm{Ci} / \mathrm{ml} \\ \text { Average } & \text { Peak } \\ ---1 .-- & ---16 \\ 7.8 \mathrm{E}-16 & 2.0 \mathrm{E}-15 \\ -5.2 \mathrm{E}-17 & 4.3 \mathrm{E}-16 \\ -2.4 \mathrm{E}-18 & 3.0 \mathrm{E}-17 \\ -3.2 \mathrm{E}-17 & 2.2 \mathrm{E}-17 \\ -1.3 \mathrm{E}-17 & 3.0 \mathrm{E}-17 \\ 7.4 \mathrm{E}-18 & 3.0 \mathrm{E}-17 \\ -3.0 \mathrm{E}-17 & 1.9 \mathrm{E}-17 \\ 3.6 \mathrm{E}-17 & 1.9 \mathrm{E}-16 \\ -9.6 \mathrm{E}-17 & 4.4 \mathrm{E}-18 \\ 1.1 \mathrm{E}-18 & 5.4 \mathrm{E}-18 \\ 4.1 \mathrm{E}-18 & 8.1 \mathrm{E}-18 \\ 4.1 \mathrm{E}-17 & 5.5 \mathrm{E}-17 \\ 3.0 \mathrm{E}-16 & 3.2 \mathrm{E}-15 \\ 3.0 \mathrm{E}-14 & 7.1 \mathrm{E}-14\end{array}$


166\%21/96 11:21

296-A-29: Ai! AN TANKS (E901)

01/01:95 to $12 / 31 / 95$

Page 1

\begin{tabular}{|c|c|c|c|c|c|c|}
\hline 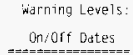 & 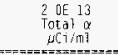 & 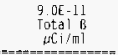 & $\begin{array}{l}\text { Collect } \\
\text { Time } \\
\text { Hours } \\
\text { Hours }\end{array}$ & $\begin{array}{l}\text { Sample } \\
\text { Flowkate } \\
\text { CFM }\end{array}$ & $\begin{array}{l}\text { Sample } \\
\text { Volute } \\
\mathrm{ft3} \\
=-=\end{array}$ & $\begin{array}{l}\text { Stack } \\
\text { Flowkate } \\
\text { CFFM } \\
\text { CF= }\end{array}$ \\
\hline 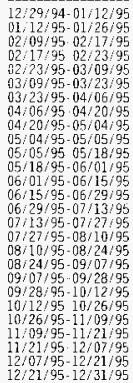 & 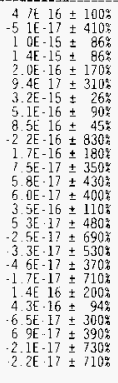 & 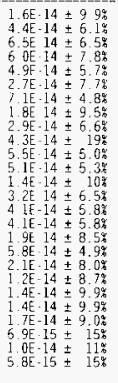 & $\begin{array}{l}335.3 \\
332.0 \\
392.0 \\
192.9 \\
194.0 \\
334.3 \\
333.9 \\
333.9 \\
337.9 \\
335.9 \\
26.5 \\
309.3 \\
325.2 \\
331.9 \\
337.7 \\
338.0 \\
328.0 \\
336.0 \\
336.0 \\
340.3 \\
499.6 \\
338.3 \\
335.7 \\
332.9 \\
294.0 \\
378.0 \\
341.8 \\
0.0\end{array}$ & $\begin{array}{r}=-1.0 \\
2.0 \\
2.0 \\
2.0 \\
2.0 \\
2.0 \\
1.9 \\
1.9 \\
2.0 \\
2.5 \\
2.8\end{array}$ & $\begin{array}{l}=74000 \\
390000 \\
39000 \\
23000 \\
17000 \\
39000 \\
35000 \\
38000 \\
40000 \\
50000 \\
4500 \\
46000 \\
44000 \\
46000 \\
45000 \\
45000 \\
44000 \\
47000 \\
46000 \\
47000 \\
69000 \\
47000 \\
45000 \\
47000 \\
40000 \\
55000 \\
49000 \\
490\end{array}$ & \\
\hline
\end{tabular}


(16/?)/96 11:21

296 A-?9: ALL AN TANKS (K901

$1: 01: 9$; to $12: 31: 95$

Pragc 1

\begin{tabular}{|c|c|c|c|c|c|c|c|c|c|}
\hline $\begin{array}{l}\text { Warning Levels: } \\
\text { On/Off Dales }\end{array}$ & $\begin{array}{l}9.9 \mathrm{E} \cdot 12 \\
5 \mathrm{r}-90 \\
\mu \mathrm{C}-1 \mathrm{imi}\end{array}$ & $\begin{array}{l}3.0 \mathrm{E} \cdot 1] \\
\mathrm{RU} \cdot 106 \\
\forall \mathrm{Ci} i \mathrm{~m}] \\
===-==\end{array}$ & 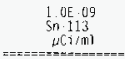 & 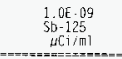 & 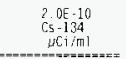 & 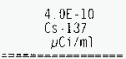 & $\begin{array}{l}\text { 5. } \mathrm{BE}-11 \\
\mathrm{Eu} \cdot 152 \\
\mu \mathrm{C} / \mathrm{m} 1\end{array}$ & 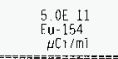 & $\begin{array}{l}\text { 3. } 05-10 \\
\mathrm{Eu}-155 \\
\mu \mathrm{C} i / \mathrm{ml}\end{array}$ \\
\hline 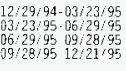 & $\begin{array}{l}\text { 5. OE } 16 \pm \pm 12 \% \\
2.0 E-15 \pm 20 \% \\
2.5 E-165 \pm 35 \% \\
1.4 E-16 \pm 35\end{array}$ & $\begin{array}{r}-2.1 \mathrm{E} \cdot 17 \pm 3000 \\
4.3 \mathrm{E} \cdot 16 \pm 1708 \\
\cdot 1.9 \mathrm{E} \cdot 16 \pm 375 \% \\
-45 \mathrm{E} \cdot 16 \pm 1608\end{array}$ & $\begin{array}{r}2.7 E \cdot 17 \pm 290 \% \\
1.2 E 17 \pm 720 \% \\
-3.9 E .17 \pm 2889 \\
3.0 E .17 \pm 330 \%\end{array}$ & $\begin{array}{r}-4.6 \mathrm{E} \cdot 17 \pm 4259 \\
5.6 \mathrm{E}-18+25098 \\
-1.2 \mathrm{E}-16 \pm 1539 \\
2.25-17 \pm 840^{\circ}\end{array}$ & $\begin{array}{rl}-1.9 E-17 & \pm 3900 \\
3.0 E-17 & \pm 260 \% \\
-2.7 E-17 & \pm 30 \% \\
4.0 E & 17 \pm 240 \%\end{array}$ & 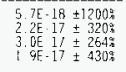 & $\begin{array}{r}6.1 \bar{E}-17 \pm 2000 \\
-5 \mathrm{E}-17 \pm 2208 \\
-2.8 \mathrm{~B}-17 \pm 4178 \\
1.9 \mathrm{E}-17 \pm 680 \%\end{array}$ & 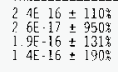 & $\begin{aligned}-1.6 \mathrm{E}-16 \pm 1108 \\
-2.2 \mathrm{E}-17 \pm 7803 \\
4.4 \mathrm{E}-18 \\
2.16 \pm 100 \% \\
2.16 \pm 100 \%\end{aligned}$ \\
\hline
\end{tabular}

06/21/96 11:21

295. A-29. ALL AN TANKS (YOQ1)

01/01/95 to $12 / 31 / 95$

Page 2

\begin{tabular}{|c|c|c|c|c|c|c|c|}
\hline $\begin{array}{l}\text { Warning levels: } \\
\text { Onloff Dates }\end{array}$ & $\begin{array}{l}\text { 3. BEE } 14 \\
\mathrm{PU} \cdot 23 \mathrm{~B} \\
\mathrm{WC \textrm {C } / \mathrm { m } ]}\end{array}$ & 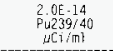 & 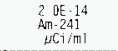 & $\begin{array}{l}\text { Collect } \\
\text { I ime } \\
\text { Hours }\end{array}$ & $\begin{array}{l}\text { Somple } \\
\text { Flowfate } \\
\text { CFM }\end{array}$ & $\begin{array}{c}\text { Sample } \\
\text { volume } \\
\mathrm{ft} 3\end{array}$ & $\begin{array}{c}\text { Stack } \\
\text { FlowRate } \\
\text { CFM }\end{array}$ \\
\hline $\begin{array}{l}12 / 29 / 94-03 / 23 / 95 \\
03 / 23995-06 / 29 / 95 \\
06 / 29 / 95-09 / 28 / 95 \\
09: 28 / 95-12 / 21 / 95\end{array}$ & 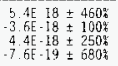 & 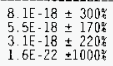 & $\begin{array}{l}3.4 \mathrm{E} .17 \pm 65 \% \\
4 . \mathrm{E} \cdot 11 \pm 50 \pi \\
2.5 \mathrm{E}-17 \pm 800 \\
5.5 \mathrm{E} .17 \pm 50 \%\end{array}$ & $\begin{array}{l}2011.3 \\
2337.7 \\
2179.2 \\
2022.0\end{array}$ & $\begin{array}{l}2.0 \\
2.2 \\
2.3 \\
2.3\end{array}$ & $\begin{array}{l}240000 \\
310000 \\
300000 \\
280000\end{array}$ & $\begin{array}{l}930 \\
930 \\
930 \\
920\end{array}$ \\
\hline
\end{tabular}

$5 Y M B O L$ KEY. * concentration exceeds hiarning tevel

? Reported MOA is negative.

n. Stack + lowkate of previous sample 


\section{WHC-SD-EN-TI-308 Rev.0}

296-A-30: 241-AN ALL ANNULI (E903)

$01 / 01 / 95$ through $12 / 31 / 95$

Total System Flow: $9.2 \mathrm{E}+10$ Liters $=3.3 \mathrm{E}+09$ Cubic Ft

The following factor(s) has (or have) been applied to the radionuclide concentrations used in the calculation of this release to account for sampling filter efficiency, line and bend loss, and humidity. The concentrations vary inversely with the size of the factor $(\mathrm{s})$.

Particulates: $\quad 0.55$
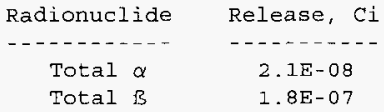

$\begin{array}{cc}\begin{array}{c}\text { Concentrations, } \mu \mathrm{Ci} / \mathrm{ml} \\ \text { Average }\end{array} & \text { Peak } \\ -1 .--- & ----- \\ \text { 1.2E-16 } & 4.6 \mathrm{E}-16 \\ \text { 1.1E-15 } & 4.3 \mathrm{E}-15\end{array}$


06/21:96 11:21

\begin{tabular}{|c|c|c|}
\hline $\begin{array}{l}\text { Warning levels: } \\
\text { On/Off Dates }\end{array}$ & $\begin{array}{c}\text { 2.DE-14 } \\
\text { Total a } \\
p C i=\mathrm{ml}\end{array}$ & $\begin{array}{l}9.0 E-12 \\
\text { Tot, al } \\
\mu C \mathrm{i} / \mathrm{mil}\end{array}$ \\
\hline 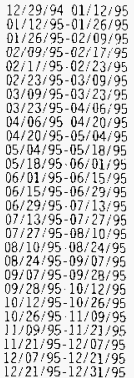 & 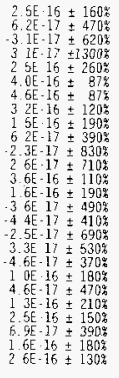 & 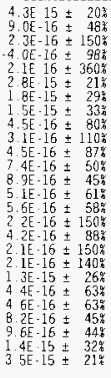 \\
\hline
\end{tabular}

296-A-30: 241-AN ALI ANNULI (E903)

conlect Sample Sample stack
Time flowkate volume flowkat

\begin{tabular}{|c|c|c|c|}
\hline $\begin{array}{l}\text { Time } \\
\text { Hours }\end{array}$ & $\begin{array}{l}\text { F7owriate } \\
\text { FFit }\end{array}$ & $\begin{array}{l}\text { valume } \\
\text { tt3 }\end{array}$ & Fiowllat \\
\hline 335.3 & 2.0 & 80000 & \\
\hline $\begin{array}{l}332.4 \\
336.9\end{array}$ & $\begin{array}{l}2.0 \\
2.1\end{array}$ & $\begin{array}{l}39000 \\
43000\end{array}$ & \\
\hline 92. & 2.5 & $\begin{array}{l}79000 \\
29000\end{array}$ & \\
\hline & $\begin{array}{l}2.4 \\
2.6\end{array}$ & $\begin{array}{l}21000 \\
520000\end{array}$ & \\
\hline 4.2 & 2.3 & 46000 & \\
\hline 305. & 2. & 47000 & \\
\hline $\begin{array}{l}335.9 \\
335.9\end{array}$ & $\begin{array}{r}2.3 \\
2.2\end{array}$ & $\begin{array}{l}470000 \\
430000\end{array}$ & 630 \\
\hline 336.6 & $\begin{array}{l}2.2 \\
2.3\end{array}$ & $\begin{array}{l}44000 \\
43000\end{array}$ & 53 \\
\hline & $\begin{array}{l}2.2 \\
2.2\end{array}$ & 45000 & \\
\hline 332. & $\begin{array}{l}2.3 \\
2.3\end{array}$ & $\begin{array}{l}46000 \\
45000\end{array}$ & 63 \\
\hline 336. & 2.3 & 46000 & 63 \\
\hline 340 & 2.3 & 47000 & t3it \\
\hline 338 & $\begin{array}{l}2.5 \\
2.5\end{array}$ & $\begin{array}{l}6000 \\
51000\end{array}$ & \\
\hline $\begin{array}{l}335 \\
332 .\end{array}$ & $\begin{array}{l}2.4 \\
2.4\end{array}$ & $\begin{array}{l}49000 \\
47000\end{array}$ & \\
\hline & $\frac{3}{2} 3$ & 41000 & \\
\hline 1 . & 2. & 4300 & \\
\hline & & & \\
\hline
\end{tabular}

$01 / 01 ; 95$ to 12:31/95

$\rho_{\text {age }}$ 


\section{WHC-SD-EN-TI-308 Rev.0}

WHC ENVIRONMENTAL RELEASE SUMMARY

296-A-40: 241-AP TANK FARM EXHAUSTER (E013, E028)

01/01/95 through $12 / 31 / 95$

Total System Flow: $1.4 \mathrm{E}+10$ Liters $=5.0 \mathrm{E}+08$ Cubic Ft

\begin{tabular}{|c|c|c|}
\hline Radionuclide & Halflife & Release, $\mathrm{Cl}$ \\
\hline---------- & $-+-+--\infty----$ & $---\cdots--\infty$ \\
\hline${ }^{90} \mathrm{Sr}$ & 29.12 Years & 4. $3 E-09$ \\
\hline${ }^{106} \mathrm{Ru}$ & 368 Days & $8.1 E-07$ \\
\hline${ }^{113} \mathrm{Sn}$ & 115 Days & $9.8 \mathrm{E}-08$ \\
\hline${ }^{125} \mathrm{Sb}$ & 2.8 Years & $2.0 \mathrm{E}-07$ \\
\hline${ }^{129} \mathrm{I}$ & $1.57 \mathrm{E}+7$ Years & $1.6 \mathrm{E}-05$ \\
\hline${ }^{134} \mathrm{Cs}$ & 2.1 Years & $4.5 E-10$ \\
\hline${ }^{2.37} \mathrm{Cs}$ & 30 Years & $2.7 \mathrm{E}-08$ \\
\hline${ }^{1.52} \mathrm{Eu}$ & 13.3 Years & 1. $4 E-09$ \\
\hline${ }^{154} \mathrm{Eu}$ & 8.8 Years & ND \\
\hline${ }^{155} \mathrm{Eu}$ & 4.96 Years & ND \\
\hline${ }^{238} \mathrm{Pu}$ & 87.8 Years & ND \\
\hline $239 / 240 \mathrm{Pu}$ & 24,131 Years* & $5.8 E-11$ \\
\hline${ }^{24} \mathrm{I}_{\mathrm{Am}}$ & 432 Years & 4. $9 E-10$ \\
\hline Total $\alpha$ & $\mathrm{n} / \mathrm{a}$ & $6.6 \mathrm{E}-09$ \\
\hline Total B & $\mathrm{n} / \mathrm{a}$ & 1. $2 \mathrm{E}-07$ \\
\hline
\end{tabular}

- The halflife shown for Total Uranium is the halflife of ${ }^{238} \mathrm{U}$. The halflife shown for mixed ${ }^{239 / 240} \mathrm{Pu}$ is the halfiife of ${ }^{239} \mathrm{Pu}$. 
06:?1/95 11:21

\begin{tabular}{|c|c|c|}
\hline Onutf Dates & & \\
\hline 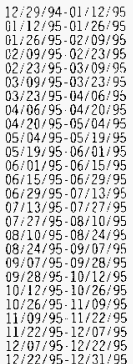 & 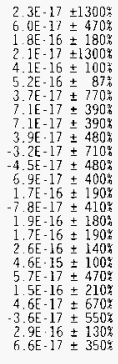 & 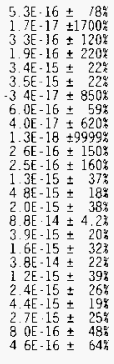 \\
\hline
\end{tabular}

296-A 4U: 241 AF 1ANK F ARM EXHAUST (EO13

\begin{tabular}{|c|c|c|c|}
\hline $\begin{array}{l}\text { Collect } \\
\text { Time } \\
\text { Hours }\end{array}$ & $\begin{array}{c}\text { Sample } \\
\text { Flompate } \\
\text { CFM }\end{array}$ & $\begin{array}{l}\text { Sample } \\
\text { volume } \\
\mathrm{ft3}\end{array}$ & $\begin{array}{l}\text { Stack } \\
\text { Flompante } \\
\text { CFFM }\end{array}$ \\
\hline 335.0 & $==-=$ & $=====$ & 950 \\
\hline 308 & 2.0 & 41000 & $\begin{array}{l}950 \\
950\end{array}$ \\
\hline 332 & 2.0 & 40000 & 9500 \\
\hline $\begin{array}{l}336.2 \\
334.4\end{array}$ & $\begin{array}{l}2.0 \\
2.0\end{array}$ & $\begin{array}{l}41000 \\
41000\end{array}$ & $\begin{array}{l}950 \\
950\end{array}$ \\
\hline 336.6 & 20 & 41000 & 9500 \\
\hline $\begin{array}{l}534.2 \\
337.2 \\
340\end{array}$ & & 41000 & 950 \\
\hline 340. & & $\begin{array}{l}41000 \\
41000\end{array}$ & $\begin{array}{l}950 \\
950\end{array}$ \\
\hline 313 & 9 & 36000 & 950 \\
\hline 339.2 & 2. & $\begin{array}{l}38000 \\
41000\end{array}$ & $\begin{array}{l}950 \\
950\end{array}$ \\
\hline 332.0 & & 41000 & 950 \\
\hline$\frac{192 .}{336}$ & $\frac{2.2}{2} 0$ & $\begin{array}{r}25000 \\
41000\end{array}$ & 950 \\
\hline 334. & . & 42000 & 950 \\
\hline 336 . & & $\begin{array}{r}42000 \\
3600\end{array}$ & $\begin{array}{l}950 \\
950\end{array}$ \\
\hline $\begin{array}{l}33.3 . \\
335 .\end{array}$ & & 41600 & 950 \\
\hline $\begin{array}{l}3.55 \\
337\end{array}$ & & $\begin{array}{l}42000 \\
42000\end{array}$ & $\begin{array}{l}550 \\
950\end{array}$ \\
\hline $\begin{array}{l}307 \\
366\end{array}$ & 2.1 & 39000 & 950 \\
\hline & & & \\
\hline & 0 & & 950 \\
\hline
\end{tabular}

^s MIA exceeds Warming level
01:01:95 to $12: 31: 95$

Page 1

? Reported MDA is negative

A Stack Flowiate of previous sample 


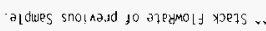

$\begin{array}{ll}2 & 092 \\ z & 9591\end{array}$

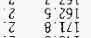

$\begin{array}{lll}2 & 580 T \\ 2 & 5991 \\ 2 & 9995\end{array}$

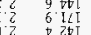

$-\frac{2}{2} \quad 8.021$

1. 691
2.945
. 291
2.291

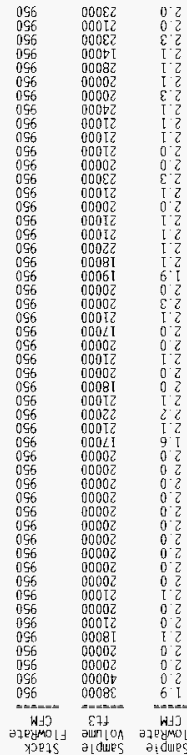

Yर०स 75

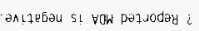

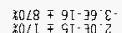

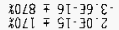

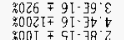

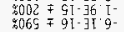

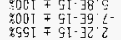

p. 99

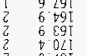

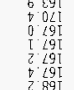

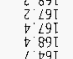

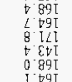

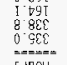

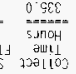

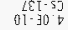

96: [ะ/2L 07 ต6/TO/T)

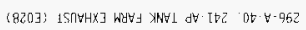

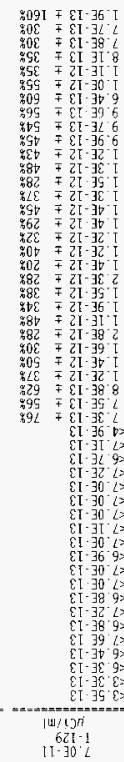

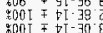

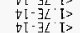

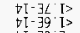

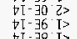

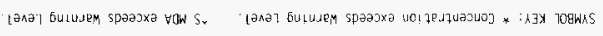

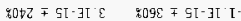

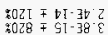

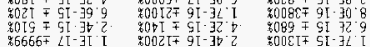

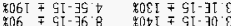

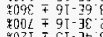

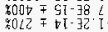

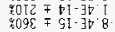

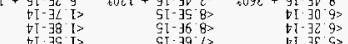

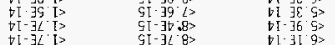

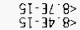

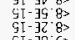

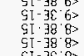

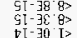

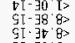

$5 \mathrm{II}-30.65$
$\mathrm{SI}-36.6$

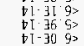

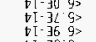

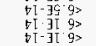

t. 389
ti $38<$ 
WHC-SD-EN-TI-308 Rev.0

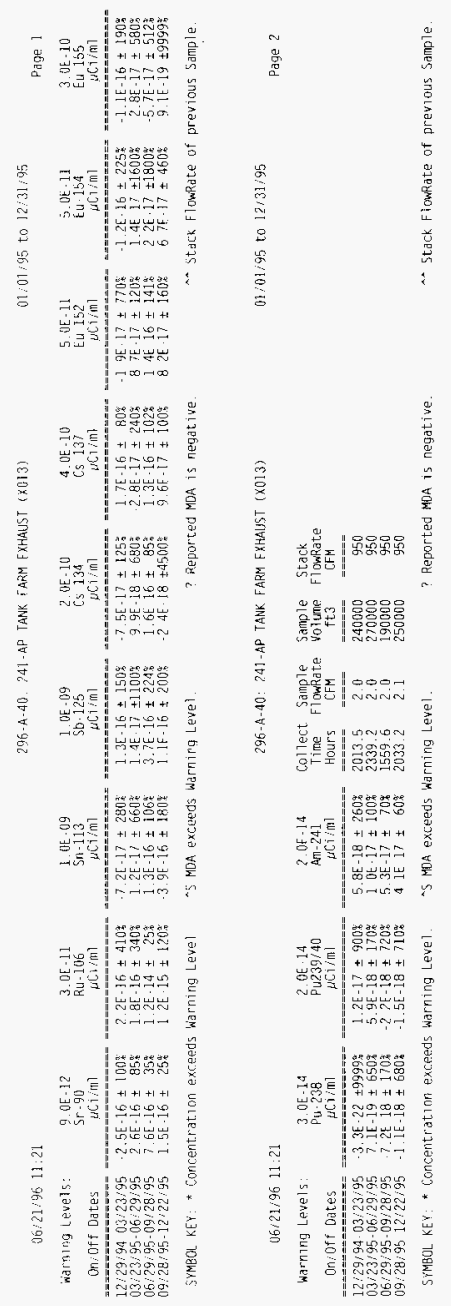




\section{WHC-SD-EN-TI-308 Rev.0}

WHC ENVIRONMENTAL RELEASE SUMMARY

296-A-41: 241-AP TANK FARM ANNULUS (E015)

$01 / 01 / 95$ through $12 / 31 / 95$

Total System Flow: 1.5E+11 Liters $=5.3 \mathrm{E}+09$ Cubic Ft

The following factor(s) has (or have) been applied to the radionuclide concentrations used in the calculation of this release to account for sampling filter efficiency, line and bend loss, and humidity. The concentrations vary inversely with the size of the factor $(s)$.

$$
\text { Particulates: } \quad 0.80
$$

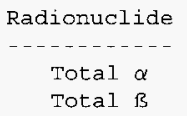

Radionuclide

Total $\alpha$

Total $\beta$

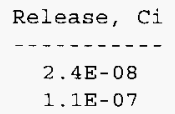

$\begin{array}{cc}\text { Concentrations, } \mu \mathrm{Ci} / \mathrm{ml} \\ \text { Average } & \text { Peak } \\ ------ & ----- \\ 1.3 \mathrm{E}-16 & 4.7 \mathrm{E}-15 \\ 6.1 \mathrm{E}-16 & 3.6 \mathrm{E}-15\end{array}$


295 A 41: 241. AP TANK FARM ANNULUIS (E015)
06:21:96 11:2i

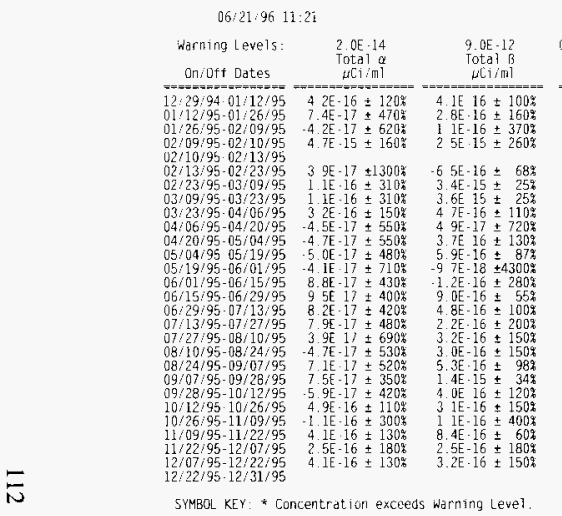

$\bar{N}$
0):12:95 to 12:31:95 Page 


\section{WHC-SD-EN-TI-308 Rev.0}

WHC ENVIRONMENTAL RELEASE SUMMARY

296-B-28: 244-BX SALT WELL RECEIVER \& ANN. (E886, X886)

01/01/95 through 12/31/95

Total System Flow: $4.2 \mathrm{E}+09$ Liters $=1.5 \mathrm{E}+08$ Cubic Ft

The following factor(s) has (or have) been applied to the radionuclide concentrations used in the calculation of this release to account for sampling filter efficiency, line and bend loss, and humidity. The concentrations vary inversely with the size of the factor (s).

Particulates:

\section{Radionuclide}

$90 \mathrm{Sr}$

${ }^{106} \mathrm{Ru}$

${ }^{113} \mathrm{Sn}$

$125 \mathrm{Sb}$

${ }^{134} \mathrm{Cs}$

${ }^{137} \mathrm{Cs}$

${ }^{152} \mathrm{Eu}$

${ }^{154} \mathrm{Eu}$

$155 \mathrm{Eu}$

${ }^{238} \mathrm{Pu}$

$239 / 240 \mathrm{Pu}$

$241_{\mathrm{Am}}$

Total $\alpha$

Total $\beta$
Release, $\mathrm{Ci}$

1. $2 \mathrm{E}-08$

ND

4. $2 \mathrm{E}-10$

4. $8 \mathrm{E}-10$

ND

2. $7 \mathrm{E}-09$

3. $1 \mathrm{E}-10$

ND

4. 2 E- 10

ND

$7.1 \mathrm{E}-11$

1. $3 \mathrm{E}-10$

1. $8 \mathrm{E}-09$

1. $7 \mathrm{E}-08$
0.72

$\begin{array}{rc}\begin{array}{c}\text { Concentrations, } \mu \mathrm{Ci} / \mathrm{ml} \\ \text { Average }\end{array} & \text { Peak } \\ ---1 .---- \\ 2.1 \mathrm{E}-15 & 7.9 \mathrm{E}-15 \\ -1.5 \mathrm{E}-16 & 3.9 \mathrm{E}-16 \\ 7.3 \mathrm{E}-17 & 3.3 \mathrm{E}-16 \\ 8.3 \mathrm{E}-17 & 2.2 \mathrm{E}-16 \\ -1.3 \mathrm{E}-17 & 1.0 \mathrm{E}-16 \\ 4.6 \mathrm{E}-16 & 7.2 \mathrm{E}-16 \\ 5.4 \mathrm{E}-17 & 1.1 \mathrm{E}-16 \\ -4.8 \mathrm{E}-17 & 1.0 \mathrm{E}-17 \\ 7.3 \mathrm{E}-17 & 2.7 \mathrm{E}-16 \\ -2.1 \mathrm{E}-18 & 5.4 \mathrm{E}-18 \\ 1.2 \mathrm{E}-17 & 4.7 \mathrm{E}-17 \\ 2.2 \mathrm{E}-17 & 5.4 \mathrm{E}-17 \\ 3.1 \mathrm{E}-16 & 1.0 \mathrm{E}-15 \\ 2.9 \mathrm{E}-15 & 1.1 \mathrm{E}-14\end{array}$




\section{WHC-SD-EN-TI-308 Rev.0}
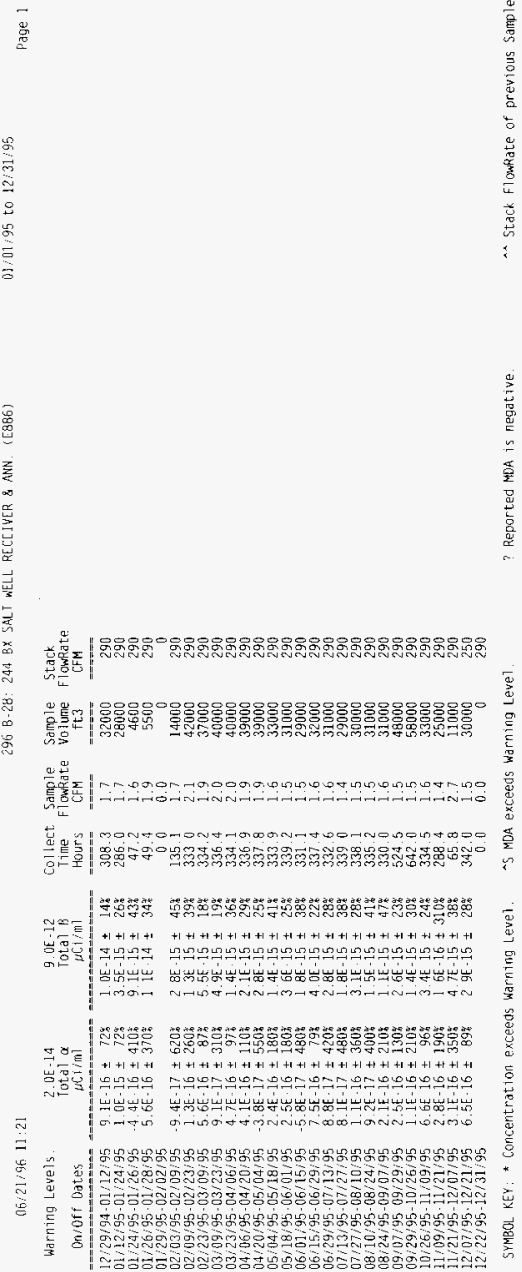
[6:? ?]:96 1!:?1

296-B-28: 296-B-?R STACK SAMPI FR ( $\times 886)$

(11:01:95 to $12 / 31: 95$

\begin{tabular}{|c|c|c|c|c|c|c|c|c|c|}
\hline $\begin{array}{l}\text { Warning Levets: } \\
\text { Un/uft Odtes }\end{array}$ & $\begin{array}{l}9.0 E-12 \\
5 r-90 \\
\mu i j / \text { ml }\end{array}$ & 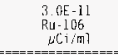 & $\begin{array}{c}1.0 \mathrm{AE} \cdot 09 \\
5 \mathrm{n}-113 \\
\mu[\mathrm{Ci} / \mathrm{m}]\end{array}$ & $\begin{array}{c}1.0 \mathrm{E}-09 \\
\mathrm{sb}-125 \\
\mu \mathrm{Ci} ; \mathrm{m}]\end{array}$ & 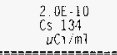 & $\begin{array}{l}\text { 4. BE }-10 \\
\text { Cs } 137 \\
\mu C i / m 1\end{array}$ & 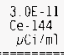 & $\begin{array}{l}5.0 \mathrm{~F}-11 \\
\mathrm{Eu}-152 \\
\mu \mathrm{Ci} / \mathrm{ml}\end{array}$ & $\begin{array}{l}5 . G E \cdot 11 \\
E u-154 \\
\mu C i / \mathrm{ml}\end{array}$ \\
\hline $\begin{array}{l}12,29 / 94-0323 ; 55 \\
03 / 23 / 45-06 / 29 / 95 \\
06 / 29 / 95-09 / 29 / 95 \\
09 / 29 / 95 \quad 12 / 21 / 95\end{array}$ & $\begin{array}{rl}-4.4 \mathrm{E}-16 & \pm 160 \% \\
7 \cdot 3 \mathrm{E}-16 \pm & \pm 0 \% \\
7 \cdot 9 \mathrm{E}-15 \pm & \pm 15 \% \\
2.3 \mathrm{E} & 17 \pm 260 \%\end{array}$ & 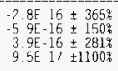 & $\begin{array}{rl}3 & 3 E-16 \pm 85 \% \\
-1.0 E-17 & \pm 1000 \\
7 & 8 E-17 \pm 163 \% \\
63 E & 17 \pm 200 \%\end{array}$ & $\begin{array}{r}1.5 \mathrm{E} .17 \pm 15000 \\
1.0 \mathrm{E}-16 \pm 2000 \\
-1.1 \mathrm{EE}-17 \pm 23000 \\
2.2 \mathrm{E}-16 \pm 30 \%\end{array}$ & 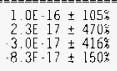 & 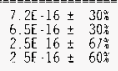 & & 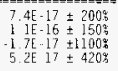 & $\begin{array}{r}9.8 \mathrm{E}-17 \pm 3300 \\
-1.4 \mathrm{E}-17 \pm 200 \% \\
-9.9 \mathrm{E}-17 \pm 379 \% \\
1.0 \mathrm{E}-17 \pm 3800 \%\end{array}$ \\
\hline
\end{tabular}

06:21/96 11:21

296-B-28: 296-B-28 STACK SAMPI FF $(X 886)$

01/01:95 to 12/31:95

Page 2

\begin{tabular}{|c|c|c|c|c|c|c|c|c|}
\hline $\begin{array}{l}\text { wiorning Levels: } \\
\text { On/off Dates }\end{array}$ & 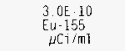 & 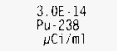 & $\begin{array}{l}2, U E-14 \\
P_{U} U 239 / 40 \\
\mu C i / 91\end{array}$ & $\begin{array}{l}2.9 E-14 \\
\text { An }-241 \\
U C 1 / \mathrm{m}]\end{array}$ & $\begin{array}{l}\text { Collect } \\
\text { Time } \\
\text { Hours }\end{array}$ & $\begin{array}{l}\text { Sample } \\
\text { Flowkrate } \\
\text { CFH }\end{array}$ & $\begin{array}{l}\text { Sample } \\
\text { volume } \\
\text { ft3 }\end{array}$ & $\begin{array}{l}\text { Stack } \\
\text { F7owRate } \\
\text { CFM }\end{array}$ \\
\hline $\begin{array}{l}12 / 29 / 94-03 / 23 / 95 \\
03 / 23 / 95-06 / 29 / 95 \\
06 / 29 / 9509 / 29 / 95 \\
09 / 29 / 95-12 / 21 / 95\end{array}$ & $\begin{array}{r}32 \mathrm{E}-17 \pm 550 \% \\
-2.5 \mathrm{E}-17 \pm 900 \% \\
1.4 \mathrm{E}-17 \pm \pm 1800 \% \\
\frac{1}{7 \mathrm{E}-16 \pm 110 \%}\end{array}$ & $\begin{array}{l}\text { 1. } 6 \mathrm{E}-17 \pm 110 \% \\
2.7 \mathrm{E}-18 \pm 240 \% \\
3.2 \mathrm{E}-18 \pm 670 \% \\
5.4 \mathrm{E}-18 \pm 270 \%\end{array}$ & $\begin{array}{l}4.0 E-18 \pm 1900 \% \\
5.6 E-18 \pm 170 \% \\
-2.118 \pm \pm 20 \% \\
4.7 E-17 \pm 70^{\circ}\end{array}$ & $\begin{array}{l}6.3 \mathrm{E}-18 \pm 240 \% \\
4.6 \mathrm{E}-18 \pm 300 \% \\
2.3 \mathrm{E}-17 \pm 120 \% \\
5.4 \mathrm{E}-17 \pm 70 \%\end{array}$ & $\begin{array}{l}1829.6 \\
2350.4 \\
2199.7 \\
1673.6\end{array}$ & $\begin{array}{l}1.8 \\
1.7 \\
1.5 \\
1.6\end{array}$ & $\begin{array}{l}200000 \\
240000 \\
200000 \\
160000\end{array}$ & $\begin{array}{l}270 \\
290 \\
290 \\
280\end{array}$ \\
\hline
\end{tabular}

SYMBO KEY: * Concentratinn exceeds warning Level.

¿S MOA exceeds warning Level.

?. Reoorted MDA is negative.

^ Stack Flowfiate of previous Särole 


\section{WHC-SD-EN-TI-308 Rev.0}

WHC ENVIRONMENTAL RELEASE SUMMARY

296-C-5: 244-CR VAULT CELL \& VESSET, VENT (E069, X059)

$01 / 01 / 95$ through $12 / 31 / 95$

Total System Flow: $7.5 \mathrm{E}+10$ Liters $=2.6 \mathrm{E}+09$ Cubic Ft

The following factor(s) has (or have) been applied to the radioruclide concentrations used in the calculation of this release to account for sampling filter efficiency, line and bend loss, and humidity. The concentrations vary inversely with the size of the factor $(s)$.

$$
\text { Particulates: } \quad 0.83
$$

${ }^{90} \mathrm{Sr}$
${ }^{106} \mathrm{Ru}$
${ }^{113} \mathrm{Sn}$
${ }^{125} \mathrm{Sb}$
${ }^{134} \mathrm{Cs}$
${ }^{137} \mathrm{Cs}$
${ }^{152} \mathrm{Eu}$
${ }^{154} \mathrm{Eu}$
${ }^{155} \mathrm{Eu}$
${ }^{238} \mathrm{Pu}$
$239^{240} \mathrm{Pu}$
${ }^{241} \mathrm{Am}$
Total $\alpha$
Total $\beta$

Release, Ci
$2.7 E-07$
ND
$1.7 E-09$
$6.4 E-09$
$4.1 E-09$
$2.8 E-07$
ND
$3.5 E-11$
$3.4 E-09$
$4.6 E-10$
$3.7 E-09$
$2.2 E-09$
$2.5 E-08$
$1.1 E-06$

$\begin{array}{cc}\text { Concentrations, } \mu \mathrm{Ci} / \mathrm{ml} \\ \text { Average } & \text { Peak } \\ --0 \mathrm{E}-15 & 5.1 \mathrm{E}-15 \\ -4.0 \mathrm{E}-16 & 1.1 \mathrm{E}-17 \\ 1.9 \mathrm{E}-17 & 1.0 \mathrm{E}-16 \\ 7.1 \mathrm{E}-17 & 9.3 \mathrm{E}-17 \\ 4.5 \mathrm{E}-17 & 8.5 \mathrm{E}-17 \\ 3.1 \mathrm{E}-15 & 4.1 \mathrm{E}-15 \\ -6.6 \mathrm{E}-18 & 4.7 \mathrm{E}-17 \\ 3.9 \mathrm{E}-19 & 1.6 \mathrm{E}-16 \\ 3.8 \mathrm{E}-17 & 9.1 \mathrm{E}-17 \\ 5.2 \mathrm{E}-18 & 1.3 \mathrm{E}-17 \\ 4.1 \mathrm{E}-17 & 1.5 \mathrm{E}-16 \\ 2.4 \mathrm{E}-17 & 3.3 \mathrm{E}-17 \\ 2.8 \mathrm{E}-16 & 1.4 \mathrm{E}-15 \\ 1.3 \mathrm{E}-14 & 4.5 \mathrm{E}-14\end{array}$


$06 / 21 / 9611: 21$

\begin{tabular}{|c|c|c|}
\hline $\begin{array}{l}\text { Warming Levels } \\
\text { On/off Dates }\end{array}$ & $\begin{array}{l}\text { 2. OE-14 } \\
\text { Total } \alpha \\
\text { ot } 1 / \pi 1 / 1\end{array}$ & $\begin{array}{l}9.0 E-17 \\
\text { Total } \\
\mu C i \text { mi }\end{array}$ \\
\hline 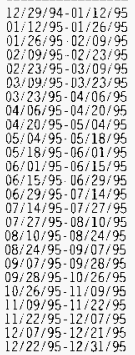 & 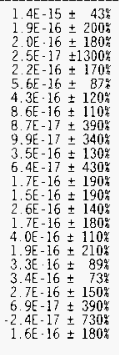 & 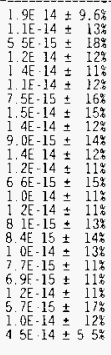 \\
\hline
\end{tabular}

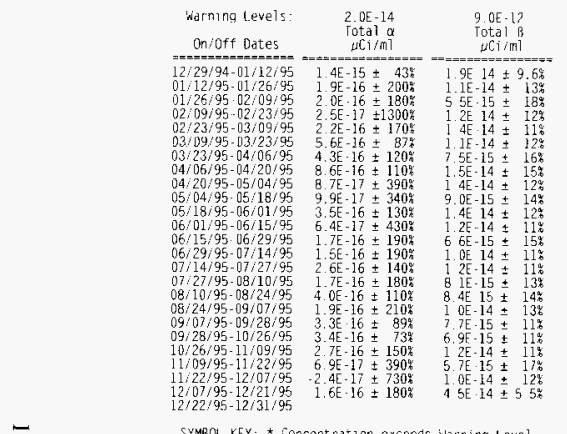

SYMBOH KEY: * Concentration exceeds warning Lovol
296.Г-5. 244 CA VAULT CELL \& YESSEL VENT (E069)

\begin{tabular}{|c|c|c|c|}
\hline $\begin{array}{l}\text { Col lect } \\
\text { Tl ale } \\
\text { Hours }\end{array}$ & $\begin{array}{l}\text { Sample } \\
\text { Flowirate } \\
\text { CFM }\end{array}$ & $\begin{array}{l}\text { Semple } \\
\text { yolume } \\
\mathrm{ft} 3\end{array}$ & $\begin{array}{l}\text { Stack } \\
\text { Plowkate } \\
\text { CFM }\end{array}$ \\
\hline $\begin{array}{r}335.0 \\
333.4 \\
274.1 \\
331.5 \\
338.1 \\
338.1 \\
333.5 \\
154.8 \\
335.7 \\
335.4 \\
333.3 \\
328.6 \\
338.9 \\
356.3 \\
312.9 \\
335.5 \\
343.9 \\
330.1 \\
499.6 \\
682.6 \\
331.0 \\
310.1 \\
357.5 \\
341.7 \\
0.0\end{array}$ & $\begin{array}{l}1.8 \\
1.8 \\
2.3 \\
1.8 \\
1.8 \\
1.9 \\
1.7 \\
2.0 \\
1.6 \\
1.7 \\
1.7 \\
2.1 \\
2.1 \\
2.2 \\
2.2 \\
2.3 \\
1.8 \\
1.7 \\
2.1 \\
2.0 \\
2.2 \\
2.2 \\
2.2 \\
2.2 \\
0.0\end{array}$ & $\begin{array}{r}36000 \\
37000 \\
38000 \\
36000 \\
37000 \\
30000 \\
33000 \\
18000 \\
33000 \\
335000 \\
35000 \\
42000 \\
42000 \\
477000 \\
42000 \\
46000 \\
33000 \\
34000 \\
64000 \\
82000 \\
43000 \\
40000 \\
460000 \\
45000 \\
0\end{array}$ & $\begin{array}{l}5000 \\
5000 \\
5000 \\
5000 \\
5000 \\
5000 \\
5000 \\
5000 \\
5000 \\
5000 \\
5000 \\
5000 \\
5000 \\
5000 \\
5000 \\
5500 \\
5000 \\
5000 \\
5000 \\
5000 \\
5000 \\
5000 \\
5000 \\
5000 \\
5000\end{array}$ \\
\hline
\end{tabular}

$\checkmark$ MISA exceeds Warning Level

? Reported MDA is negative
01/61/95 to 12:31/95

Page : 
$96: 21: 96 \quad 11 \cdot 21$

256.C.5. 244-CR VAUL T CFLI \& YESSEL YENT (XOE9)

01:01/95 to $12: 31: 95$

Page 1

\begin{tabular}{|c|c|c|c|c|c|c|c|c|c|}
\hline $\begin{array}{l}\text { Warning Levels: } \\
\text { onoff lates } \\
==========\end{array}$ & $\begin{array}{l}9.0 F-17 \\
5-90 \\
u C 1 / \mathrm{ml} \\
======\end{array}$ & 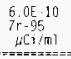 & 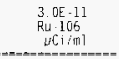 & 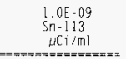 & $\begin{array}{c}1.0 \mathrm{E}-09 \\
\mathrm{Sb}-125 \\
\mu \mathrm{Cl} 1 / \mathrm{ml}\end{array}$ & 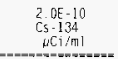 & $\begin{array}{l}4.0 \mathrm{E}-10 \\
\mathrm{Cs} 137 \\
\mu[\mathrm{CT} / \mathrm{ml}\end{array}$ & 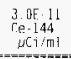 & 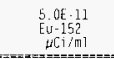 \\
\hline 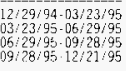 & $\begin{array}{l}\text { 2. } 7 E-15 \pm 12 \% \\
\text { 2. } 8 E-15 \pm 15 \% \\
\text { 1. } 3 E-15 \pm 15 \% \\
\text { 3. } 1 \mathrm{E} 15 \pm 18 \%\end{array}$ & & 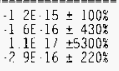 & $\begin{aligned} 1.0 E \cdot 16 \pm 2400 \\
-6.9 F .17 \pm 1500 \\
3.7 E-17 \pm 2120 \\
2.3 E=17 \pm 360 \%\end{aligned}$ & 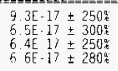 & $\begin{array}{l}8.5 E-17 \pm 175 \\
1.11-17 \pm 7204 \\
7.8 E \cdot 17 \pm 842 \\
1.5 E-17 \pm 4809\end{array}$ & $\begin{array}{l}2.9 E-17 \pm 20 \% \\
4.0 E .25 \pm 200 \\
3.7 E-15 \pm 190 \\
4.1 F-15 \pm 200\end{array}$ & & $\begin{array}{r}2.9 \mathrm{E}-17 \pm 560 \\
4.7 \mathrm{E}-17 \pm 270 \mathrm{z} \\
-5.5 \mathrm{E}-17 \pm 216 \% \\
3.2 \mathrm{E}-18 \pm 4400 \%\end{array}$ \\
\hline
\end{tabular}

SYMBOL KEY + Concentration exceeds in'arning Level.

๑5 MDA exceeds Warming l evel

? Reported MDA is neadive.

- stack Flowkate of previous sample.

06/21:96 $11: 31$

296 C.5. 244-CR YAULT CEII \& VESSEL YENT (X069)

$01 / 01 / 95$ to $12 / 31 / 95$

Page 2

\begin{tabular}{|c|c|c|c|c|c|c|c|c|c|}
\hline $\begin{array}{l}\text { Wharning Levols: } \\
\text { Onioff Dates }\end{array}$ & 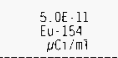 & $\begin{array}{l}3.0 E-10 \\
E u .155 \\
\omega C i / m 1\end{array}$ & $\begin{array}{l}3.0 \mathrm{E} \cdot 14 \\
\mathrm{P} \mathbf{\mathrm { J }}-238 \\
\mu \mathrm{C} \mathbf{i} / \mathrm{ml}\end{array}$ & 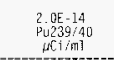 & $\begin{array}{l}2.0 E-14 \\
\text { Ann-24L } \\
\mu \mathrm{CI} / \mathrm{mI}\end{array}$ & $\begin{array}{l}\text { Collect } \\
\text { Tine } \\
\text { Hours }\end{array}$ & $\begin{array}{c}\text { Sample } \\
\text { Flomkate } \\
\text { CFM }\end{array}$ & $\begin{array}{c}\text { Sannole } \\
\text { volume } \\
\text { ft3 }\end{array}$ & $\begin{array}{l}\text { Stack } \\
\text { Flowkate } \\
\text { CFM }\end{array}$ \\
\hline $\begin{array}{l}12 / 29 / 94-03 / 23 / 95 \\
03 / 23 / 95-06 / 29 / 95 \\
069 / 29 / 95-69 / 28 / 95 \\
09 / 28 / 95-12 / 21 / 95\end{array}$ & $\begin{array}{r}-2.4 \mathrm{E}-16 \pm 105 \% \\
-5.8 \mathrm{E}-19 \pm 9999 \% \\
4.6 \mathrm{E}-17 \pm 458 \% \\
1.6 \mathrm{E}-16 \pm 140 \%\end{array}$ & $\begin{array}{l}9 \mathrm{WE} 17 \pm 250 \% \\
2 \mathrm{LE}-17 \pm 900 \% \\
7.2 \mathrm{E}-18 \pm 2300 \% \\
5 \mathrm{PE}-17 \pm 370 \%\end{array}$ & $\begin{array}{rl}1 & 3 E \cdot 17 \pm 200 \% \\
-12 E & 28 \pm 150 \% \\
6.4 E \cdot 18 \pm 190 \% \\
3.7 E \cdot 18 \pm 300\end{array}$ & $\begin{array}{l}1.5 E \cdot 16 \pm 48 \% \\
13 E \pm 17 \pm 00 \% \\
3.8 E 18 \pm 30 \% \\
8.5 E-18 \pm 170 \%\end{array}$ & $\begin{array}{l}1.8 \mathrm{E}-17 \pm 1009 \\
2.1 \mathrm{E} .17 \pm 90 \mathrm{a} \\
2.5 \mathrm{E}-17 \pm 908 \\
3.3 \mathrm{E} 17 \pm 909\end{array}$ & $\begin{array}{l}1950.2 \\
2160.3 \\
2178.3 \\
2023.0\end{array}$ & $\begin{array}{l}1.9 \\
1.9 \\
1.8 \\
2.1\end{array}$ & $\begin{array}{l}220000 \\
240000 \\
270000 \\
260000\end{array}$ & $\begin{array}{l}50010 \\
55000 \\
5000 \\
5000\end{array}$ \\
\hline
\end{tabular}




\section{WHC-SD-EN-TI-308 Rev.0}

$06 / 15 / 96$

WHC ENVIRONMENTAL RELEASE SUMMARY

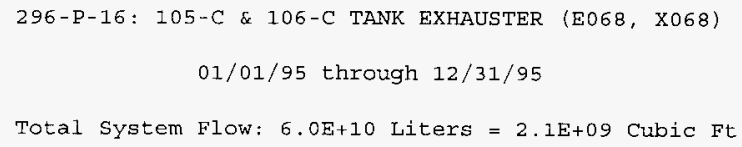
to account for sampling filter efficiency, Iine and bend loss, and humidity. The concentrations vary inversely with the size of the factor $(s)$.

\begin{tabular}{|c|c|c|c|}
\hline \multirow[b]{2}{*}{ Radionuclide } & ulates: & \multicolumn{2}{|l|}{0.73} \\
\hline & Release, $\mathrm{Ci}$ & $\begin{array}{c}\text { Concentrati } \\
\text { Average }\end{array}$ & 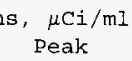 \\
\hline$------\cdots,--$ & ---------- & ------- & ----- \\
\hline${ }^{90} \mathrm{Sr}$ & 4. $2 E-07$ & $5.1 E-15$ & 1. $0 E-14$ \\
\hline${ }^{106} \mathrm{Ru}$ & $\mathrm{ND}$ & $-5.9 E-17$ & $2.0 E-16$ \\
\hline${ }^{113} \mathrm{Sn}$ & ND & $-2.1 E-16$ & $5.2 E-17$ \\
\hline $125 \mathrm{Sb}$ & 3. $7 \mathrm{E}-09$ & 4. $5 \mathrm{E}-17$ & $2.2 E-16$ \\
\hline${ }^{134} \mathrm{Cs}$ & $\mathrm{ND}$ & $-2.5 E-17$ & 1. $3 E-17$ \\
\hline${ }^{137} \mathrm{CSS}$ & $1.4 \mathrm{E}-06$ & $1.7 E-14$ & $4 \cdot 5 E-14$ \\
\hline${ }^{152} \mathrm{Eu}$ & 1. $7 E-09$ & $2.1 \mathrm{E}-17$ & 1. $2 \mathrm{E}-16$ \\
\hline${ }^{154} \mathrm{Eu}$ & ND & $-3 \cdot 3 E-17$ & 8. $9 E-17$ \\
\hline${ }^{155} \mathrm{Eu}$ & $4.6 E-09$ & $5.6 \mathrm{E}-17$ & $3.0 E-16$ \\
\hline${ }^{238} \mathrm{Pu}$ & 1.1E-09 & $1.3 E-17$ & $3.0 E-17$ \\
\hline 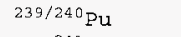 & $1.9 \mathrm{E}-10$ & $2.3 E-18$ & $2.6 \mathrm{E}-17$ \\
\hline${ }^{241} \mathrm{Am}$ & $6.8 \mathrm{E}-09$ & 8. $3 \mathrm{E}-17$ & 1. $5 E-16$ \\
\hline Total $\alpha$ & 1. $8 \mathrm{E}-08$ & $2.2 \mathrm{E}-16$ & 1. $3 \mathrm{E}-15$. \\
\hline Total B & 2. $0 \mathrm{E}-06$ & $2.4 E-14$ & $2.3 E-13$ \\
\hline
\end{tabular}


$26 / 21: 96$ 11 22

\begin{tabular}{|c|c|c|c|c|c|c|}
\hline $\begin{array}{l}\text { Iarning Levels } \\
\text { Onroff Dates }\end{array}$ & 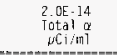 & 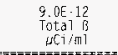 & $\begin{array}{c}\text { Collect } \\
\text { rines } \\
\text { Hours }\end{array}$ & $\begin{array}{c}\text { Sarple } \\
\text { H lowkate } \\
\text { CFM }\end{array}$ & & $\begin{array}{c}\text { Stack } \\
\text { Flownat } \\
\text { CFM }\end{array}$ \\
\hline 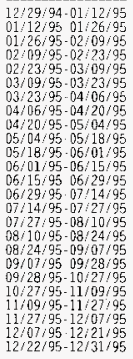 & 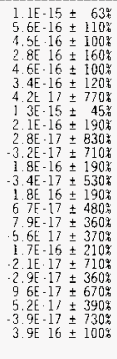 & 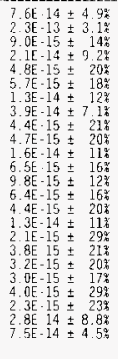 & $\begin{array}{l}330.4 \\
338.2 \\
338.1 \\
332.5 \\
310.3 \\
336.4 \\
335.5 \\
336.8 \\
322.4 \\
339.3 \\
336.0 \\
313.1 \\
335.7 \\
344.5 \\
330.1 \\
499.6 \\
700.8 \\
312.6 \\
430.8 \\
236.2 \\
332.2 \\
0.0\end{array}$ & $\begin{array}{l}1.8 \\
1.8 \\
1.7 \\
1.7 \\
1.8 \\
2.7 \\
2.2 \\
2.0 \\
19 \\
2.0 \\
1.9 \\
1.9 \\
1.9 \\
1.1 \\
2.0 \\
2.0 \\
0.0\end{array}$ & $\begin{array}{r}38000 \\
37000 \\
54000 \\
79000 \\
200000 \\
53000 \\
29000 \\
40000 \\
0\end{array}$ & $\begin{array}{l}4000 \\
4000 \\
4000 \\
4000 \\
4000 \\
4000 \\
4000 \\
4000 \\
4000 \\
4000 \\
4000 \\
4000 \\
4000 \\
4000 \\
4000\end{array}$ \\
\hline
\end{tabular}




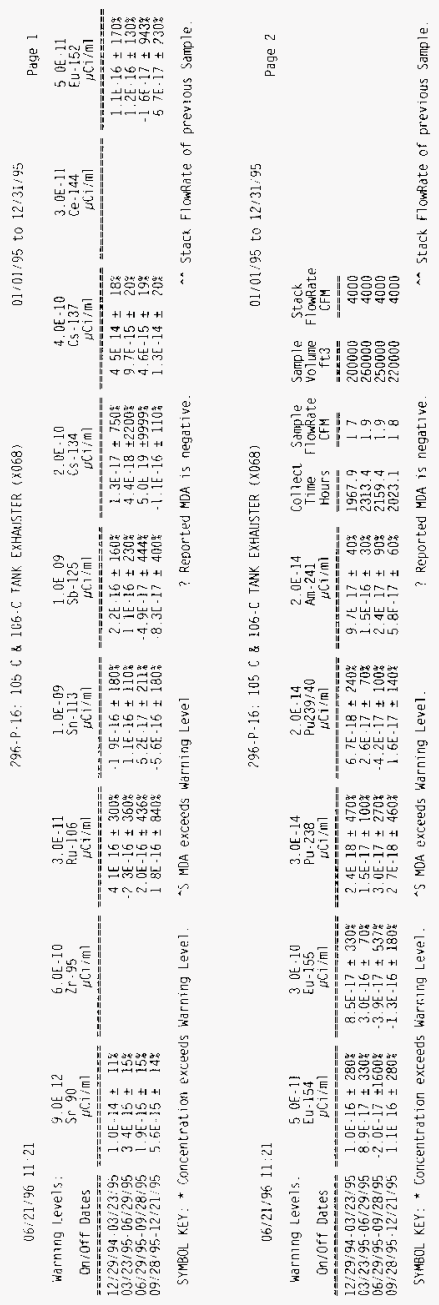




\section{WHC-SD-EN-TI-308 Rev.0}

WHC ENVIRONMENTAL RELEASE SUMMARY

296-P-31: 209-E CRITICALITY LABORATORY EXHAUST (E209)

$01 / 01 / 95$ through $12 / 31 / 95$

Total system Flow: $3.3 \mathrm{E}+10$ Liters $=1.1 \mathrm{E}+09$ Cubic Ft

The following factor(s) has (or have) been applied to the radionuclide concentrations used in the calculation of this release to account for sampling filter efficiency, line and bend loss, and humidity. The concentrations vary inversely with the size of the factor (s).

$$
\text { Particulates: } \quad 0.91
$$

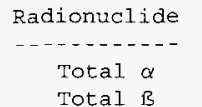
Release, Ci 6.-...-
6. $4 \mathrm{E}-09$
4. $2 \mathrm{E}-08$

\begin{tabular}{cc}
$\begin{array}{c}\text { Concentrations, } \mu \mathrm{Ci} / \mathrm{mI} \\
\text { Average }\end{array}$ & Peak \\
\hdashline$-\cdots----$ \\
$1.8 \mathrm{E}-16$ & $1.3 \mathrm{E}-15$ \\
$1.2 \mathrm{E}-15$ & $6.7 \mathrm{E}-15$
\end{tabular}


06/21:96 $31: 21$

\begin{tabular}{|c|c|c|c|c|c|c|}
\hline & 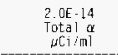 & $\begin{array}{l}9.0 \mathrm{DE} \cdot 12 \\
\text { Total } \\
\mu[\mathrm{T} / \mathrm{mi}\end{array}$ & $\begin{array}{l}\text { Collect. } \\
\text { Time } \\
\text { Hours }\end{array}$ & $\begin{array}{l}\text { Sample } \\
\text { Flowkate } \\
\text { CFH }\end{array}$ & $\begin{array}{c}\text { Sample } \\
\text { Solune } \\
\text { tit. }\end{array}$ & $\begin{array}{c}\text { Stack } \\
\text { FiowRa } \\
\text { CFM }\end{array}$ \\
\hline $\begin{array}{l}1195.10 / 1395 \\
13 / 95-10 / 26 / 95 \\
20695 .-11 / 0995 \\
09 / 95-11 / 21 / 95 \\
201 / 95-12 / 0795 \\
07 / 95.12 / 21 / 95 \\
21 / 95-12 / 31 / 95\end{array}$ & 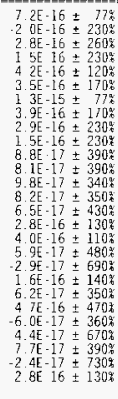 & 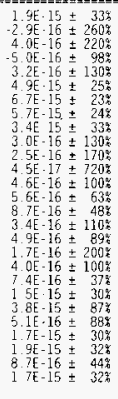 & 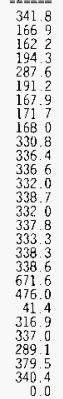 & $\begin{array}{l}2 . \\
2 . \\
2 . \\
2 . \\
2 . \\
0 .\end{array}$ & $\begin{array}{l}==== \\
38,000 \\
18000 \\
19000 \\
23000 \\
34000 \\
23000 \\
20000 \\
200000 \\
20000 \\
30000 \\
33000 \\
360000 \\
35000 \\
40000\end{array}$ & $\begin{array}{l}2 \\
2 \\
2 \\
2 \\
2 \\
2\end{array}$ \\
\hline
\end{tabular}




\section{WHC-SD-EN-TI-308 Rev.0}

WHC ENVIRONMENTAL RELEASE SUMMARY

296-P-32, 33, \& 34: ROTARY MODE CORE SAMPLERS (E301-E399) $01 / 01 / 95$ through $12 / 31 / 95$

Total System Flow: 1.2E+09 Liters $=4.3 E+07$ Cubic Ft

$\begin{array}{ccc}\text { Radionuclide } & \text { Halflife } & \text { Release, Ci } \\ { }^{90} \mathrm{Sr} & 29.12 \text { Years } & 3.0 \mathrm{E}-08 \\ { }^{105} \mathrm{Ru} & 368 \text { Days } & \mathrm{ND} \\ { }^{113} \mathrm{Sn} & 115 \text { Days } & 4.6 \mathrm{E}-11 \\ { }^{125} \mathrm{Sb} & 2.8 \text { Years } & 1.4 \mathrm{E}-09 \\ { }^{134} \mathrm{Cs} & 2.1 \text { Years } & 1.1 \mathrm{E}-09 \\ { }^{137} \mathrm{Cs} & 30 \text { Years } & 2.0 \mathrm{E}-09 \\ { }^{152} \mathrm{Eu} & 13.3 \text { Years } & 3.9 \mathrm{E}-10 \\ { }^{154} \mathrm{Eu} & 8.8 \text { Years } & 4.1 \mathrm{E}-10 \\ { }^{155} \mathrm{Eu} & 4.96 \text { Years } & 2.1 \mathrm{E}-09 \\ { }^{238} \mathrm{Pu} & 87.8 \text { Years } & \mathrm{ND} \\ 239 / 240 \mathrm{Pu} & 24131 \text { Years* } & 3.9 \mathrm{E}-10 \\ { }^{241} \mathrm{Am} & 432 \text { Years } & 8.3 \mathrm{E}-10 \\ \text { Total } \alpha & \text { n/a } & 1.9 \mathrm{E}-09 \\ \text { Total } \mathrm{B} & \text { n/a } & 6.9 \mathrm{E}-10\end{array}$

* The halflife shown for Total Uranium is the halflife of ${ }^{238} \mathrm{U}$. The halflife shown for mixed ${ }^{239 / 240} \mathrm{Pu}$ is the halflife of ${ }^{239} \mathrm{Pu}$. 
WHC-SD-EN-TI-308 Rev.0

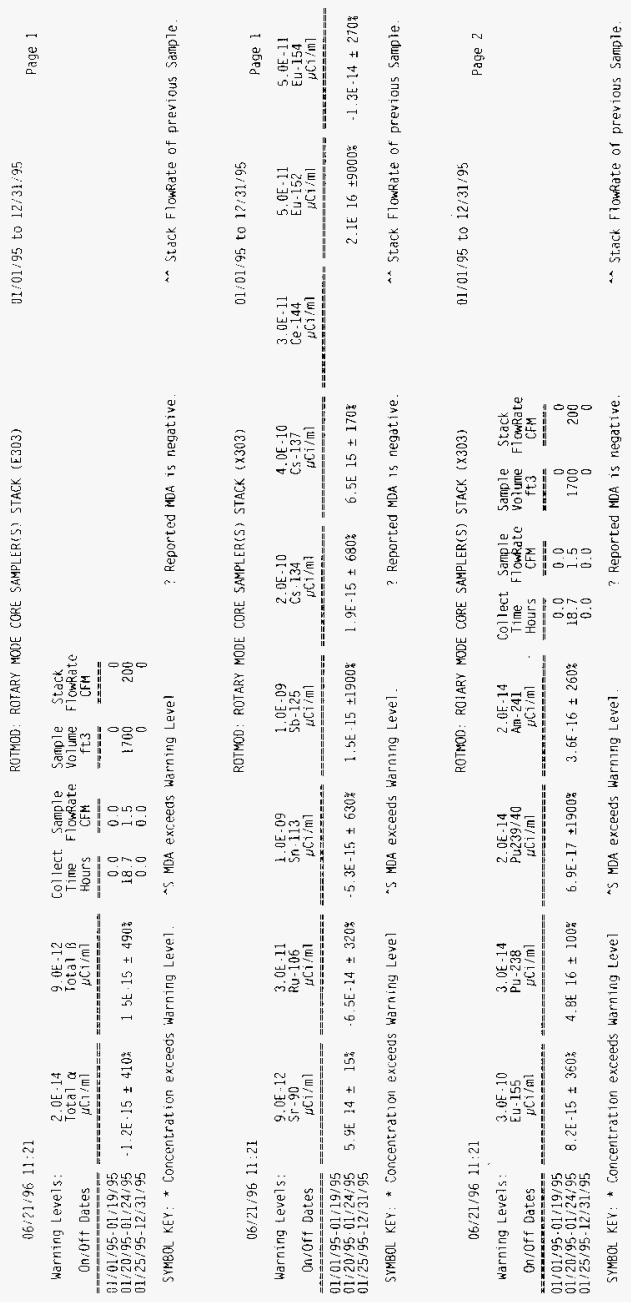


WHC-SD-EN-TI-308 Rev.0

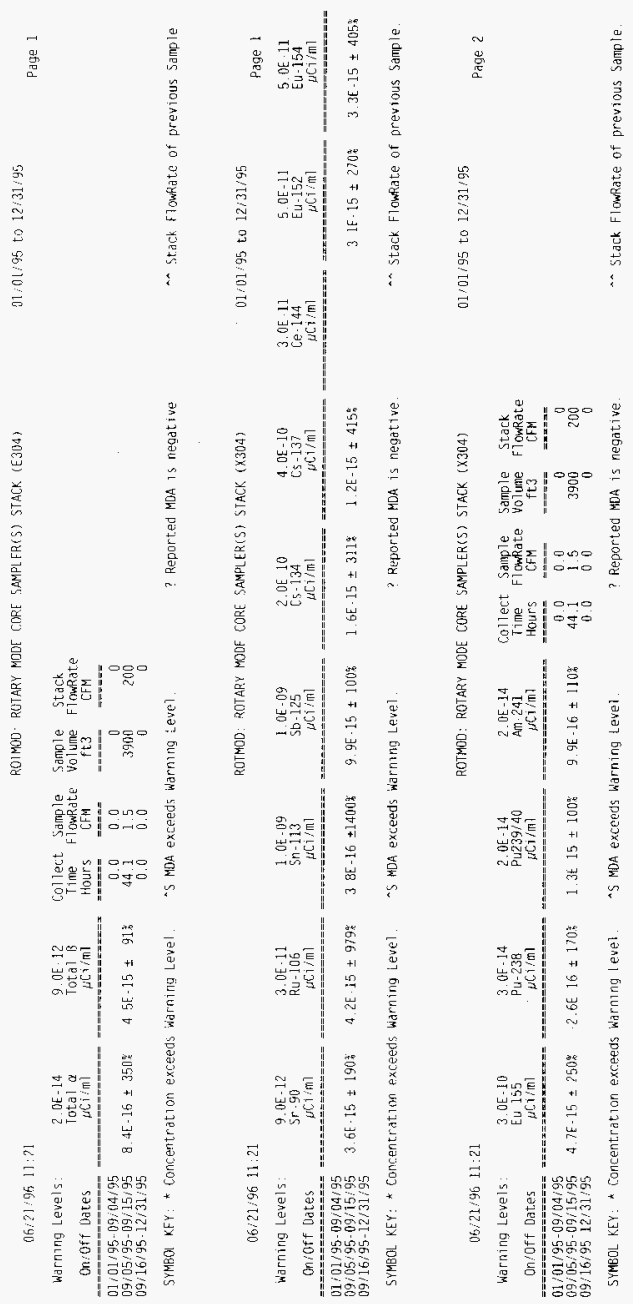




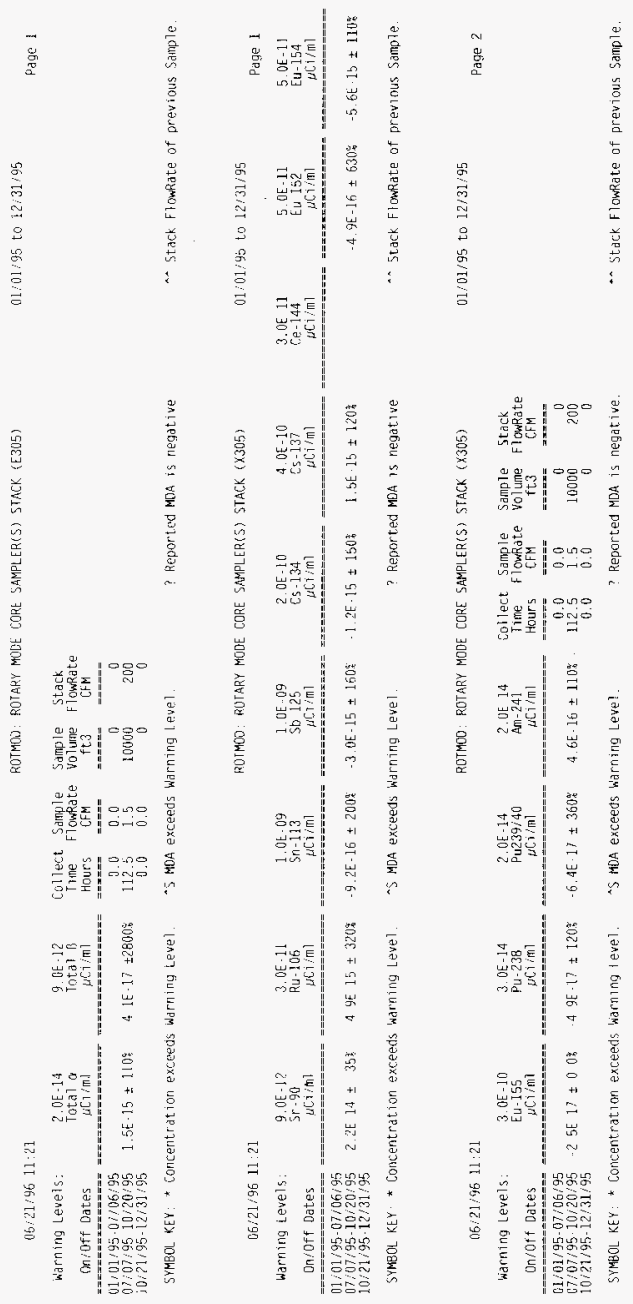




\begin{tabular}{|c|c|c|c|c|c|c|}
\hline $\begin{array}{l}\text { Namirig Levels: } \\
\text { Un:off bates }\end{array}$ & $\begin{array}{l}2.0 E \cdot 14 \\
\text { Total a } \\
\mu C+1 \mathrm{ml}\end{array}$ & $\begin{array}{l}9.05-12 \\
\text { Total } \\
\text { TCI } 1 / \mathrm{mm}\end{array}$ & $\begin{array}{l}\text { Collect } \\
\text { Time } \\
\text { Hours }\end{array}$ & 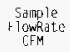 & $\begin{array}{l}\text { Sample } \\
\text { volume } \\
\text { ft } 3\end{array}$ & $\begin{array}{l}\text { Stack } \\
\text { flowkate } \\
\text { CFM }\end{array}$ \\
\hline $\begin{array}{l}01 / 95.07 / 23 / 95 \\
24 / 95.08 / 18 / 95 \\
199512 / 31 / 95\end{array}$ & E & $O E$ & $\begin{array}{r}0.0 \\
66.2 \\
0.0\end{array}$ & $\begin{array}{l}0.0 \\
1.5 \\
0.0\end{array}$ & & \\
\hline
\end{tabular}

SFMBDL. KEY: * concentratiun exceeds waining Level. 5 MDA exceeds warning Level.

$06 / 21 / 96 \quad 11: 21$

ROTMOD: ROTARY MOOE CORE SAMPLER(S) STACK (x306)

$01 / 01 ; 95$ to $12: 31 / 95$

\begin{tabular}{|c|c|c|c|c|c|c|c|c|c|}
\hline $\begin{array}{l}\text { Warning Levels: } \\
\text { Onioff Dates }\end{array}$ & 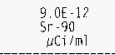 & $\begin{array}{l}\text { 3. } 0 \mathrm{E}-11 \\
\mathrm{Ru}-106 \\
\mu \mathrm{C}, 1 / \mathrm{ml}\end{array}$ & 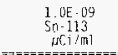 & 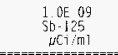 & 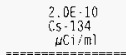 & $\begin{array}{c}40 \mathrm{OE}-10 \\
\mathrm{CS}-137 \\
\mu \mathrm{Ci} i \mathrm{ml}\end{array}$ & 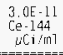 & $\begin{array}{l}5.0 \mathrm{E}-11 \\
\mathrm{E} u-152 \\
\mu 15,1 / \mathrm{m}\end{array}$ & 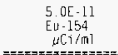 \\
\hline $\begin{array}{l}01 / 01 / 9507 / 23 / 95 \\
07 / 74 / 95.08 / 18 / 95 \\
08 / 19 / 95.12 / 31 / 95\end{array}$ & 3. $65 \quad 15 \pm 110 \%$ & $1.4 \mathrm{E} \cdot 15 \pm 1909 \%$ & $-2.9 \mathrm{E}-15 \pm 110 \%$ & 3. $9 \mathrm{E}-16 \pm 1800$ & 3. $4 E-15 \pm 1005$ & $1.2 E \quad 16 \pm 23006$ & & $7.2 E-16 \pm 700 \%$ & $-2.8 \mathrm{E}-15 \pm 370^{a}$ \\
\hline
\end{tabular}

RETMOD: ROTARY MODE CORE SAMPLER(S) STACK (X306)

$01 / 01 / 95$ 10 12/31/95

\begin{tabular}{|c|c|c|c|c|c|c|c|c|}
\hline $\begin{array}{l}\text { Warming Levels. } \\
\text { Onioff oates }\end{array}$ & $\begin{array}{l}\text { 3. } D E=10 \\
\operatorname{EU}-155 \\
\mu C i / \pi)\end{array}$ & $\begin{array}{l}3.0 E-14 \\
P U-238 \\
\forall C 1 / m T\end{array}$ & $\begin{array}{l}2.0 E-14 \\
\begin{array}{l}\mathrm{P} W 239 / 40 \\
\mu C \mathrm{C} / \mathrm{ml}\end{array}\end{array}$ & $\begin{array}{l}2.0[-14 \\
A \mathrm{Am}-241 \\
y C 7 / \mathrm{mI}\end{array}$ & $\begin{array}{l}\text { Collect } \\
\text { Inie } \\
\text { Hours }\end{array}$ & $\begin{array}{l}\text { Sample } \\
\text { Flowite } \\
\text { CFM }\end{array}$ & $\begin{array}{l}\text { Sumvle } \\
\text { volume } \\
\text { ft3 }\end{array}$ & $\begin{array}{l}\text { Stack } \\
\text { Flowfate } \\
\text { CFM }\end{array}$ \\
\hline 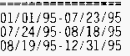 & $3.5 E-15 \pm 2|3| b \mid$ & $1.6 \mathrm{E} \cdot 16 \pm 300 x$ & $7.8 \mathrm{E}-16 \pm 120$ & $-16 \pm 1500 \%$ & $\begin{array}{r}0.0 \\
6.2 \\
0.0\end{array}$ & $\begin{array}{l}-==- \\
0.0 \\
1.5 \\
0.0\end{array}$ & $\begin{array}{l}==-= \\
5900 \\
0\end{array}$ & $\begin{array}{r}== \pm \\
000 \\
0\end{array}$ \\
\hline
\end{tabular}

^ Stack Flowkate of previous Sample 
WHC-SD-EN-TI-308 Rev.0

2.4.4 242-A Evaporator 


\section{WHC-SD-EN-TI-308 Rev.0}

WHC ENVIRONMENTAL RELEASE SUMMARY

295-A-21: 242-A BUILDING EXHAUST (E645, X645)

$01 / 01 / 95$ through $12 / 31 / 95$

Total System Flow: $3.4 \mathrm{E}+11$ Liters $=1.2 \mathrm{E}+10$ Cubic Ft

The following factor(s) has (or have) been applied to the radionuclide concentrations used in the calculation of this release to account for sampling filter efficiency, line and bend loss, and humidity. The concentrations vary inversely with the size of the factor(s).

\begin{tabular}{cccc} 
& Particulates: & 0.79 \\
& & & \\
Concentrations, $\mu \mathrm{Ci} / \mathrm{ml}$ \\
Radionuclide & Release, Ci & Average & Peak \\
\hline${ }^{90} \mathrm{Sr}$ & $-1.3 \mathrm{E}-07$ & $1.7 \mathrm{E}-15$ & $8.3 \mathrm{E}-15$ \\
${ }^{106} \mathrm{Ru}$ & $4.0 \mathrm{E}-08$ & $9.3 \mathrm{E}-17$ & $2.9 \mathrm{E}-16$ \\
${ }^{113} \mathrm{Sn}$ & $\mathrm{ND}$ & $-6.2 \mathrm{E}-17$ & $2.1 \mathrm{E}-16$ \\
${ }^{125} \mathrm{Sb}$ & $7.6 \mathrm{E}-09$ & $1.8 \mathrm{E}-17$ & $1.5 \mathrm{E}-16$ \\
${ }^{134} \mathrm{Cs}$ & $\mathrm{ND}$ & $-5.4 \mathrm{E}-18$ & $2.8 \mathrm{E}-17$ \\
${ }^{137} \mathrm{Cs}$ & $8.5 \mathrm{E}-09$ & $2.0 \mathrm{E}-17$ & $1.1 \mathrm{E}-16$ \\
${ }^{152} \mathrm{Eu}$ & $2.3 \mathrm{E}-09$ & $5.4 \mathrm{E}-18$ & $8.9 \mathrm{E}-17$ \\
${ }^{154} \mathrm{Eu}$ & $7.1 \mathrm{E}-08$ & $1.7 \mathrm{E}-16$ & $2.6 \mathrm{E}-16$ \\
${ }^{155} \mathrm{Eu}$ & $1.6 \mathrm{E}-09$ & $3.7 \mathrm{E}-18$ & $7.4 \mathrm{E}-17$ \\
$238 \mathrm{Pu}$ & $1.1 \mathrm{E}-09$ & $2.7 \mathrm{E}-18$ & $8.8 \mathrm{E}-18$ \\
$239 / 240 \mathrm{Pu}$ & $1.4 \mathrm{E}-08$ & $3.4 \mathrm{E}-17$ & $1.2 \mathrm{E}-16$ \\
${ }^{241} \mathrm{Am}$ & $1.1 \mathrm{E}-08$ & $2.5 \mathrm{E}-17$ & $4.7 \mathrm{E}-17$ \\
Total $\alpha$ & $5.1 \mathrm{E}-08$ & $1.2 \mathrm{E}-16$ & $4.7 \mathrm{E}-16$ \\
Total $\mathrm{B}$ & $3.5 \mathrm{E}-07$ & $8.2 \mathrm{E}-16$ & $4.7 \mathrm{E}-15$
\end{tabular}




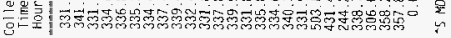

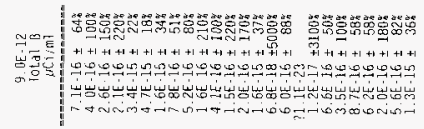




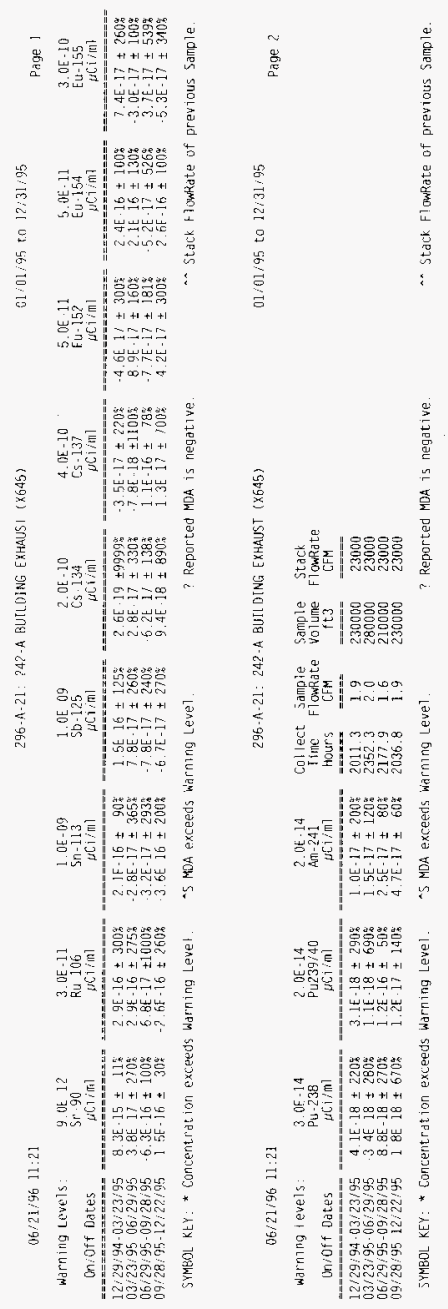


WHC-SD-EN-TI-308 Rev.0

WHC ENVIRONMENTAL RELEASE SUMMARY

$06 / 15 / 96$

296-A-22: 242-A EVAPORATOR (E643, EO01)

$01 / 01 / 95$ through $12 / 31 / 95 * * *$

Total System Flow: $9.6 \mathrm{E}+09$ Liters $=3.4 \mathrm{E}+08$ Cubic Ft

$\begin{array}{ccc}\text { Radionuclide } & \text { Halflife } & \text { Release, Ci } \\ { }^{90} \mathrm{Sr} & 29.12 \text { Years } & 5.4 \mathrm{E}-08 \\ { }^{306} \mathrm{Ru} & 368 \text { Days } & 4.8 \mathrm{E}-07 \\ { }^{113} \mathrm{Sn} & 115 \text { Days } & 4.7 \mathrm{E}-08 \\ { }^{125} \mathrm{Sb} & 2.8 \text { Years } & 7.4 \mathrm{E}-08 \\ { }^{129} \mathrm{I} & 1.57 \mathrm{E}+7 \text { Years } & 3.7 \mathrm{E}-06 \\ { }^{134} \mathrm{Cs} & 2.1 \text { Years } & 6.4 \mathrm{E}-10 \\ { }^{137} \mathrm{Cs} & 30 \text { Years } & 5.5 \mathrm{E}-10 \\ { }^{152} \mathrm{Eu} & 13.3 \text { Years } & \mathrm{ND} \\ { }^{154} \mathrm{Eu} & 8.8 \text { Years } & \mathrm{ND} \\ { }^{155} \mathrm{Eu} & 4.96 \text { Years } & 4.0 \mathrm{E}-10 \\ { }^{238} \mathrm{Pu} & 87.8 \text { Years } & 1.7 \mathrm{E}-11 \\ 239 / 240 \mathrm{Pu} & 24,131 \text { Years* } & 1.8 \mathrm{E}-10 \\ { }^{241} \mathrm{Am} & 432 \text { Years } & 4.0 \mathrm{E}-10 \\ \text { Total } \alpha & \text { n/a } & 3.4 \mathrm{E}-09 \\ \text { Total } \mathrm{B} & \text { n/a } & 4.9 \mathrm{E}-08\end{array}$

* The halflife shown for Total Uranium is the halfIife of ${ }^{238} \mathrm{U}$. The halflife shown for mixed ${ }^{239 / 240} \mathrm{Pu}$ is the halflife of ${ }^{239} \mathrm{Pu}$.

** V Values Eor one or more files projected forward to cover report period. 
$06 / 21 / 9616.21$

\begin{tabular}{|c|c|c|c|c|c|c|}
\hline $\begin{array}{l}\text { Warning ievels: } \\
\text { Onioff Dates }\end{array}$ & 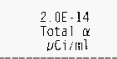 & 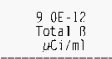 & $\begin{array}{l}\text { Colvect } \\
\text { Tinee } \\
\text { Hours }\end{array}$ & $\begin{array}{l}\text { Somple } \\
\text { Fomplate } \\
\text { CFM }\end{array}$ & $\begin{array}{c}\text { Sample } \\
\text { volure } \\
\mathrm{ft3}\end{array}$ & $\begin{array}{l}\text { Stack } \\
\text { Flowkt } \\
\text { CFMA }\end{array}$ \\
\hline 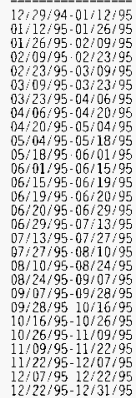 & 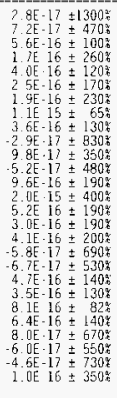 & 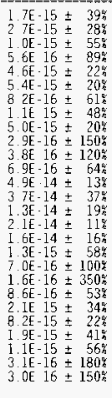 & $\begin{array}{l}==7.7 \\
331.5 \\
341.7 \\
331.4 \\
334.6 \\
337.0 \\
335.1 \\
335.2 \\
337.4 \\
339.7 \\
331.9 \\
335.9 \\
338.1 \\
100.8 \\
21.1 \\
217.7 \\
331.6 \\
335.9 \\
334.6 \\
340.2 \\
331.4 \\
506.0 \\
430.9 \\
243.5 \\
333.7 \\
309.7 \\
362.1 \\
357.8\end{array}$ & $\begin{array}{l}1.2 \\
0.8 \\
1.8\end{array}$ & $\begin{array}{l}32000 \\
340000 \\
33000 \\
31000 \\
31000 \\
32000 \\
30000 \\
32000 \\
32000 \\
35000 \\
33000 \\
32000 \\
37500 \\
1400 \\
1400 \\
14000 \\
160000 \\
200000 \\
23000 \\
23000 \\
2340000 \\
300000 \\
17000 \\
24000 \\
240000 \\
250000\end{array}$ & \\
\hline
\end{tabular}




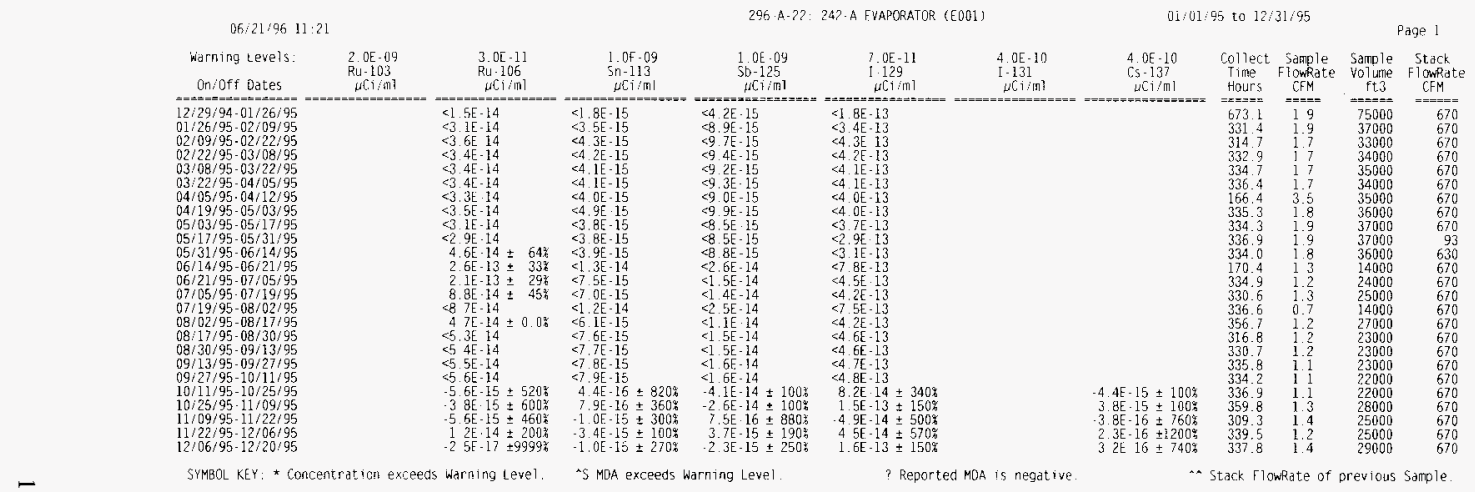




\section{WHC-SD-EN-TI-308 Rev.0}

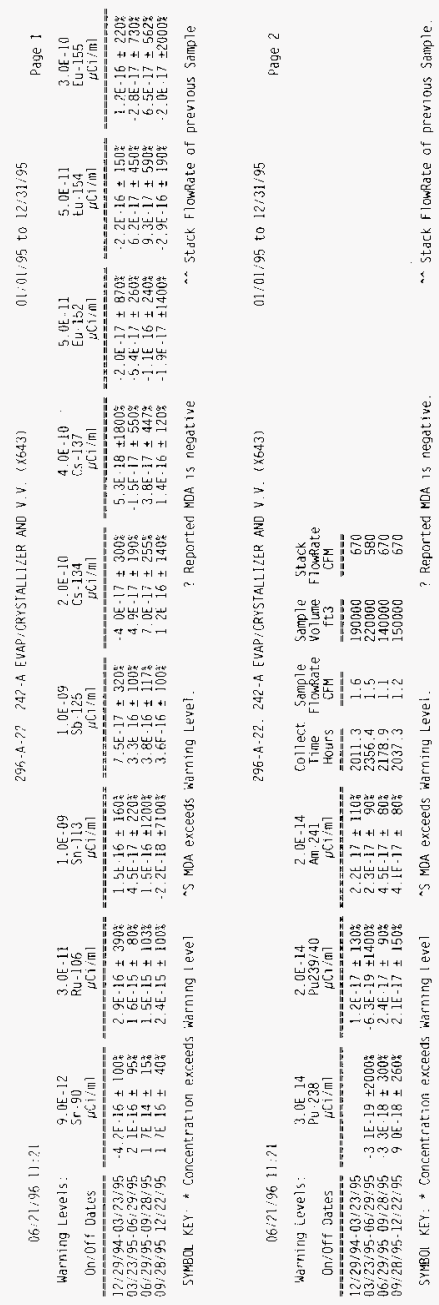


WHC-SD-EN-TI-308 Rev.0

2.4.5 Effluent Treatment Facility (ETF) 
WHC-SD-EN-TI-308

WHC ENVIRONMENTAL RELEASE SUMMARY

$06 / 14 / 96$

296-E-1: ETF Stack (E036)

01/01/95 through $12 / 31 / 95$

Total System Flow: 1.2E+11 Liters $=4.1 \mathrm{E}+09$ Cubic Ft

The following factor(s) has (or have) been applied to the radionuclide concentrations used in the calculation of this release to account for sampling filter efficiency, line and bend loss, and humidity. The concentrations vary inversely with the size of the factor $(s)$.

Particulates: $\quad 0.72$

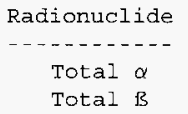

\author{
Release, Ci \\ -.-.-...... \\ 9. $6 \mathrm{E}-08$ \\ 2. $1 \mathrm{E}-07$
}

$\begin{array}{cc}\text { Concentrations, } \mu \mathrm{Ci} / \mathrm{ml} \\ \text { Average } & \text { Peak } \\ ------- & ----- \\ 6.0 \mathrm{E}-16 & 1.7 \mathrm{E}-15 \\ 1.3 \mathrm{E}-15 & 3.8 \mathrm{E}-15\end{array}$


06:21:96 11:21

\begin{tabular}{|c|c|c|c|c|c|c|}
\hline $\begin{array}{l}\text { Warrifrig Levels: } \\
\text { OniGf Dates }\end{array}$ & $\begin{array}{l}2.0 E \cdot-14 \\
\text { Total } \alpha \\
\text { utimm }\end{array}$ & 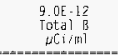 & $\begin{array}{l}\text { Collect } \\
\text { Time } \\
\text { Hours } \\
\text {-- }\end{array}$ & $\begin{array}{l}\text { Somple } \\
\text { Flowkate } \\
\text { CFM } \\
==-==\end{array}$ & $\begin{array}{l}\text { Sarple } \\
\text { volume } \\
\text { ft. } 3\end{array}$ & $\begin{array}{l}\text { Stack } \\
\text { Flowiate } \\
\text { CFM }\end{array}$ \\
\hline $\begin{array}{l}01 / 01 / 95-11 / 10 / 95 \\
11 / 11 / 95-11 / 12 / 95 \\
11 / 12 / 9511 / 22 / 95 \\
11 / 22 / 95-12 / 05 / 95 \\
12 / 05 / 95-12 / 19 / 95 \\
12 / 19 / 95-12 / 21 / 95 \\
12 / 21 / 9512 / 28 / 95 \\
12 / 28 / 95-12 / 31 / 95\end{array}$ & $\begin{array}{l}5.3 \mathrm{E} 15 \pm 110 \% \\
1.7 \mathrm{E} \cdot 16 \pm 200 \% \\
1.6 \mathrm{E} \cdot 16 \pm 200^{\circ} \\
\text { 1. } 1 \mathrm{E} \cdot 15 \pm 200 \% \\
1.15 \mathrm{E} 15 \pm 66 \%\end{array}$ & $\begin{array}{l}3.5 E \cdot 16 \pm 140 \% \\
5.8 E \cdot 16 \pm 61 \% \\
\text { L.0E. } 15 \pm 40 \% \\
1.75 \cdot 15 \pm 140 \% \\
3.9 E 15 \pm 31 \%\end{array}$ & $\begin{array}{r}0.0 \\
0.0 \\
233.0 \\
313.4 \\
336.3 \\
47.4 \\
155.1 \\
0.9\end{array}$ & $\begin{array}{l}0.0 \\
0.0 \\
2.0 \\
2.1 \\
2.1 \\
2.1 \\
2.1 \\
0.1\end{array}$ & $\begin{array}{r}0 \\
29000 \\
39000 \\
43000 \\
5100 \\
20000 \\
0\end{array}$ & $\begin{array}{l}5500 \\
55000 \\
55000 \\
55000 \\
55000 \\
55000 \\
55000\end{array}$ \\
\hline
\end{tabular}

SMBOL KEY: * Concentration excecds warnirig Leve!

"S MDA exceeds warning Level
296. E-1: ETF Stact: $\{\leqslant 036$

11/01/95 to $12 / 31: 95$

Page 1

$?$ Reported MDA is negativo.

a stack flowrate of previous sampie. 
WHC-SD-EN-TI-308 Rev.0

This page intentionally left blank. 
WHC-SD-EN-TI-308 Rev.0

2.5200 West Area

2.5.1 222-S Laboratory 


\section{WHC-SD-EN-TI-308 Rev.0}

$06 / 15 / 96$

WHC ENVIRONMENTAL RELEASE SUMMARY

296-S-16: 219-S WASTE TANKS (S264)

$01 / 01 / 95$ through $12 / 31 / 95$

Total System Flow: 1.6E+09 Liters $=5.6 E+07$ Cubic Ft

The following factor(s) has (or have) been applied to the radionuclide concentrations used in the calculation of this release to account for sampling filter efficiency, line and bend loss, and humidity. The concentrations vary inversely with the size of the factor $(s)$.

$$
\text { Particulates: } \quad 0.73
$$

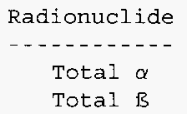

Total is

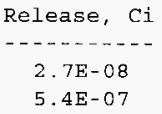

Release, $\mathrm{Ci}$

2. $7 \mathrm{E}-08$

5. $4 \mathrm{E}-07$

\begin{tabular}{cc}
$\begin{array}{c}\text { Concentrations, } \mu \mathrm{Ci} / \mathrm{ml} \\
\text { Average }\end{array}$ & Peak \\
\hline $1.2 \mathrm{E}-14$ & ----- \\
1. $9 \mathrm{E}-13$ \\
2. -13 & $3.9 \mathrm{E}-12$
\end{tabular}


$06 / 21: 96 \quad 11: 21$

295-5-16, 219-5 WASTF TA.NKS (5264)

\begin{tabular}{|c|c|c|c|c|c|c|}
\hline & 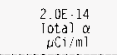 & $\begin{array}{c}9 \text { aE. } 1 \text { ? } \\
\text { Totai B } \\
\mu C i / m i\end{array}$ & $\begin{array}{l}\text { Collect } \\
\text { Time } \\
\text { Hours }\end{array}$ & $\begin{array}{l}\text { Sample } \\
\text { Flcwlate } \\
\text { CFM }\end{array}$ & $\begin{array}{c}\text { Sample } \\
\text { volume } \\
\text { ft3 }\end{array}$ & $\begin{array}{c}\text { Stack } \\
\text { FlowRate } \\
\text { CFM }\end{array}$ \\
\hline 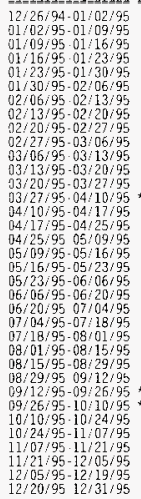 & 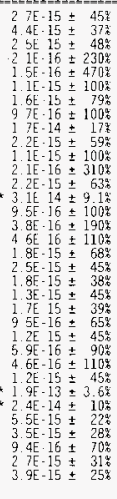 & 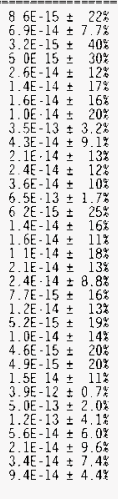 & $\begin{array}{l}===7=\pi \\
167.5 \\
169.7 \\
162.4 \\
162.5 \\
168.4 \\
172.9 \\
163.3 \\
166.5 \\
168.7 \\
172.4 \\
162.0 \\
169.4 \\
167.7 \\
338.6 \\
168.9 \\
194.9 \\
313.7 \\
166.6 \\
169.6 \\
341.4 \\
329.7 \\
334.4 \\
337.7 \\
338.7 \\
332.2 \\
330.3 \\
337.3 \\
336.2 \\
333.5 \\
338.5 \\
338.2 \\
330.6 \\
340.4 \\
335.5 \\
0.0\end{array}$ & $\begin{array}{l}1.5 \\
1.7 \\
1.7 \\
0.0\end{array}$ & 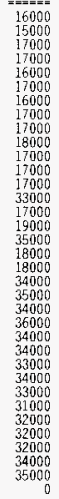 & $\begin{array}{l}10 \\
10 \\
10 \\
10\end{array}$ \\
\hline
\end{tabular}

SYMBOL KEY * Concentrdtion exceeds warning Level.
01/01:95 to $12 / 31 / 95$

Page !

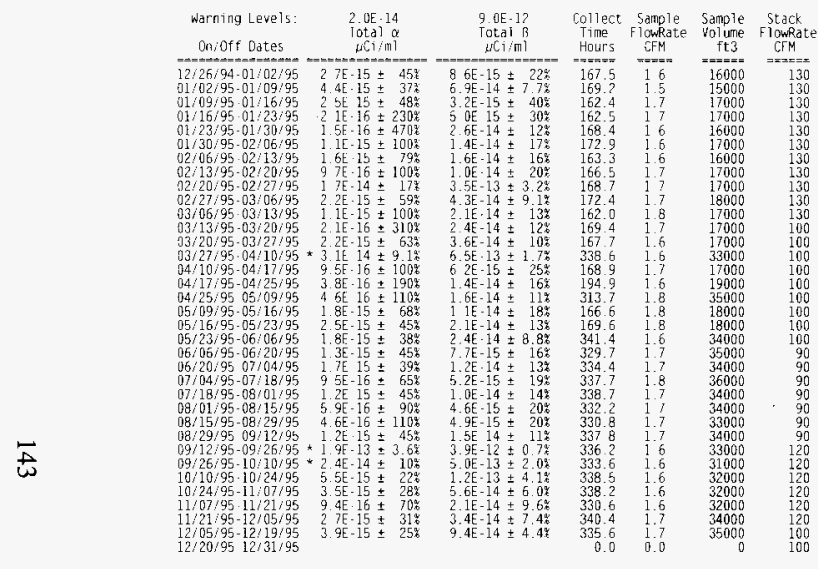

? Reportec MDA is negative. 


\section{WHC-SD-EN-TI-308 Rev.0}

WHC ENVIRONMENTAL RELEASE SUMMARY

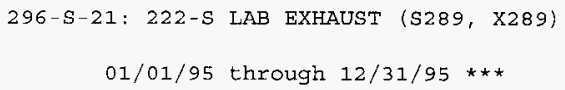
to account for sampling filter efficiency, line and bend loss, and humidity. The concentrations vary inversely with the size of the factor (s).

$$
\text { Particulates: } \quad 0.73
$$

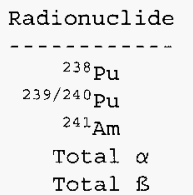

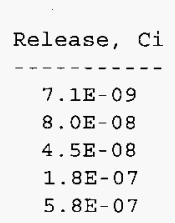

*** Values projected for period $12 / 19 / 95$ to $12 / 31 / 95$. 
$05: 21 / 96,11: 21$

\begin{tabular}{|c|c|c|c|c|c|c|}
\hline $\begin{array}{l}\text { Warning Levels: } \\
\text { On/off Eates }\end{array}$ & 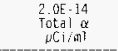 & $\begin{array}{l}9.0 \mathrm{E}-12 \\
\text { Total B B } \\
\mu \mathrm{C} i / \mathrm{mi}\end{array}$ & $\begin{array}{l}\text { Collect } \\
\text { Tine } \\
\text { Hours }\end{array}$ & $\begin{array}{l}\text { Sangle } \\
\text { Flowiate } \\
\text { CFM }\end{array}$ & $\begin{array}{l}\text { Semple } \\
\text { volume } \\
\mathrm{t}+3\end{array}$ & $\begin{array}{l}\text { Stack } \\
\text { FlowRote } \\
\text { CFM }\end{array}$ \\
\hline 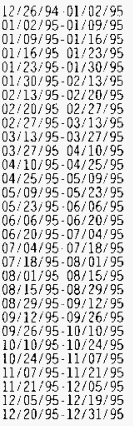 & 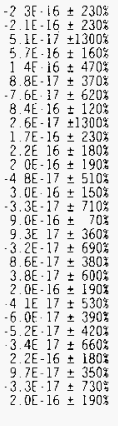 & 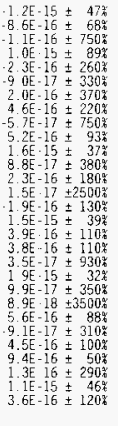 & $\begin{array}{l}139.1 \\
168.7 \\
168.4 \\
166.2 \\
169.1 \\
337.1 \\
166.9 \\
168.4 \\
336.3 \\
335.1 \\
336.7 \\
368.2 \\
318.4 \\
335.6 \\
343.1 \\
328.2 \\
334.7 \\
337.2 \\
336.5 \\
335.2 \\
336.4 \\
336.5 \\
336.1 \\
334.3 \\
337.3 \\
337.1 \\
333.2 \\
337.0 \\
338.8 \\
0.0\end{array}$ & $\begin{array}{l}19 \\
1.7 \\
1.7 \\
1.7 \\
1.7 \\
17 \\
17 \\
1.7 \\
1.7 \\
1.7\end{array}$ & $\begin{array}{l}15000 \\
17000 \\
17000 \\
17000 \\
17000 \\
35000 \\
17000 \\
17000 \\
34000 \\
34000 \\
3400 \\
37000 \\
32000 \\
34000 \\
36000 \\
3300 \\
35000 \\
35000 \\
35000 \\
36000 \\
35000 \\
35000 \\
33000 \\
36000 \\
36000 \\
35000 \\
34000 \\
35000 \\
36000 \\
0\end{array}$ & $\begin{array}{l}63000 \\
63000 \\
63000 \\
63000 \\
63000 \\
63000 \\
63000 \\
63000 \\
53000 \\
77000 \\
77000 \\
77000 \\
77000 \\
77000 \\
77000 \\
77000 \\
72000 \\
77000 \\
77000 \\
72000 \\
72000 \\
72000 \\
71000 \\
71000 \\
71000 \\
71000 \\
71000 \\
71000 \\
619000 \\
69000\end{array}$ \\
\hline
\end{tabular}

[11/101/45 to $22 / 31 / 95$

Page 1 


\section{WHC-SD-EN-TI-308 Rev.0}
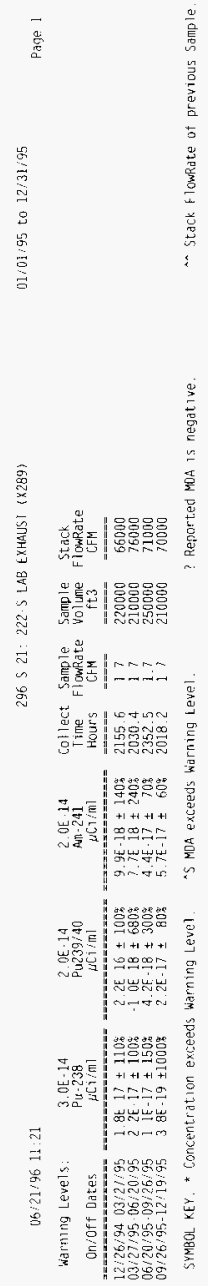
WHC-SD-EN-TI-308 Rev.0

2.5.2 T-Plant 


\section{WHC-SD-EN-TI-308 Rev.0}

WHC ENVIRONMENTAL RELEASE SUMMARY

291-T-1: 221-T CANYON \& CELLS (T785, X785)

$01 / 01 / 95$ through $12 / 31 / 95 * * *$

Total System Flow: $5.2 \mathrm{E}+11$ Liters $=1.9 \mathrm{E}+10$ Cubic Ft

The following factor(s) has (or have) been applied to the radionuclide concentrations used in the calculation of this release to account for sampling filter efficiency, line and bend loss, and humidity. The concentrations vary inversely with the size of the factor $(s)$.

Particulates: $\quad 0.73$

${ }^{90} \mathrm{Sr}$
${ }^{106} \mathrm{Ru}$
${ }^{113} \mathrm{Sn}$
${ }^{125} \mathrm{Sb}$
${ }^{134} \mathrm{Cs}$
${ }^{137} \mathrm{Cs}$
${ }^{152} \mathrm{Eu}$
${ }^{154} \mathrm{Eu}$
${ }^{155} \mathrm{Eu}$
${ }^{238} \mathrm{Pu}$
$239 / 24{ }^{20} \mathrm{Pu}$
${ }^{241} \mathrm{Am}$
Total $\alpha$
Total B

Release, Ci
$2.7 \mathrm{E}-05$
$\mathrm{ND}$
$\mathrm{ND}$
$2.0 \mathrm{E}-08$
$1.7 \mathrm{E}-08$
$1.5 \mathrm{E}-05$
$5.2 \mathrm{E}-08$
$1.3 \mathrm{E}-07$
$1.1 \mathrm{E}-07$
$3.2 \mathrm{E}-07$
$2.4 \mathrm{E}-05$
$1.8 \mathrm{E}-06$
$5.2 \mathrm{E}-05$
$1.6 \mathrm{E}-04$

$\begin{array}{cc}\text { Concentrations, } \mu \mathrm{Ci} / \mathrm{ml} \\ \text { Average } & \text { Peak } \\ ------1 & ----- \\ 3.7 \mathrm{E}-14 & 4.4 \mathrm{E}-14 \\ -8.7 \mathrm{E}-16 & -1.8 \mathrm{E}-16 \\ -5.2 \mathrm{E}-17 & 5.6 \mathrm{E}-19 \\ 2.8 \mathrm{E}-17 & 1.3 \mathrm{E}-16 \\ 2.4 \mathrm{E}-17 & 8.3 \mathrm{E}-17 \\ 2.1 \mathrm{E}-14 & 2.4 \mathrm{E}-14 \\ 7.2 \mathrm{E}-17 & 1.4 \mathrm{E}-16 \\ 1.9 \mathrm{E}-16 & 3.5 \mathrm{E}-16 \\ 1.5 \mathrm{E}-16 & 2.9 \mathrm{E}-16 \\ 4.5 \mathrm{E}-16 & 9.5 \mathrm{E}-16 \\ 3.4 \mathrm{E}-14 & 5.1 \mathrm{E}-14 \\ 2.5 \mathrm{E}-15 & 2.7 \mathrm{E}-15 \\ 7.2 \mathrm{E}-14 & 4.6 \mathrm{E}-13 \\ 2.2 \mathrm{E}-13 & 1.4 \mathrm{E}-12\end{array}$

*** Values projected for period 09/12/95 to $12 / 31 / 95$. 
06:21/96 11:21

\begin{tabular}{|c|c|c|c|c|c|c|}
\hline $\begin{array}{l}\text { Warming Levels: } \\
\text { On;off Dates }\end{array}$ & 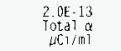 & 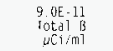 & $\begin{array}{l}\text { Col lect } \\
\text { Time } \\
\text { Hours }\end{array}$ & $\begin{array}{c}\text { Sample } \\
\text { Flowiate } \\
C+M\end{array}$ & $\begin{array}{c}\text { Sample } \\
\text { yolume } \\
\mathrm{ft} .3\end{array}$ & $\begin{array}{l}\text { Stack } \\
\text { FlowRate } \\
\text { CFM }\end{array}$ \\
\hline 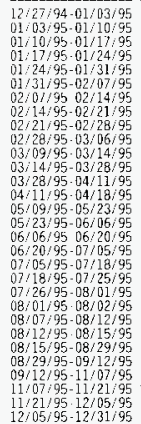 & 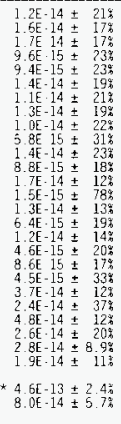 & 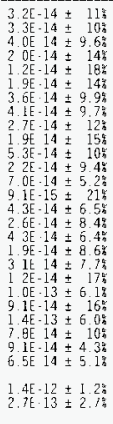 & 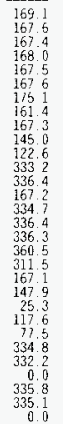 & $\begin{array}{l}1.7 \\
1.8 \\
1.7 \\
1.7 \\
1.7 \\
1.7\end{array}$ & $\begin{array}{l}18000 \\
18000 \\
17000 \\
18000 \\
18000 \\
17000 \\
18000 \\
17000 \\
17000 \\
15000 \\
12000 \\
32000 \\
33000 \\
17000 \\
34000 \\
34000 \\
34000 \\
43000 \\
34000 \\
18000 \\
16000 \\
2600 \\
13000 \\
8200 \\
36000 \\
360000 \\
0 \\
31000 \\
32000 \\
0\end{array}$ & 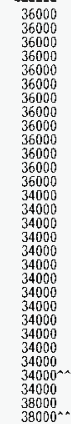 \\
\hline
\end{tabular}

J1:01:95 to 12:31:95

Page 1

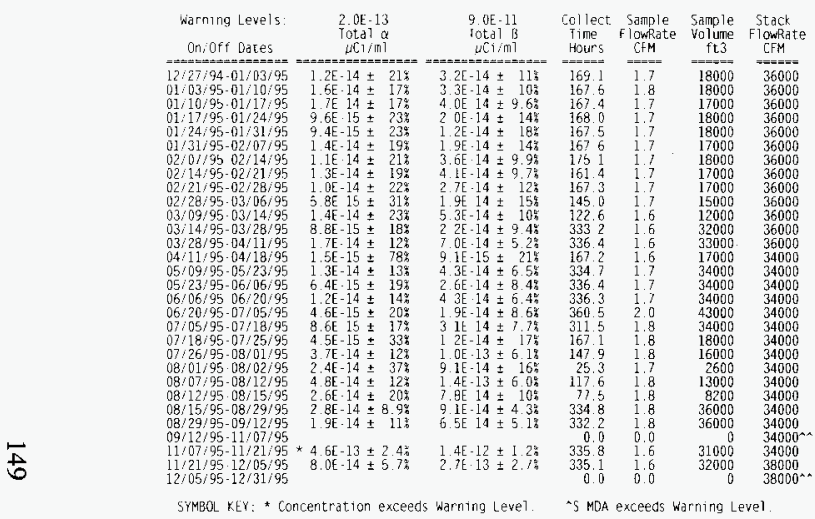

“ Stack flowRate of previous Sample 
06.? ?:96 11:?1

291 I 1: 221-T CANYON \& CELLS (X7B5)

01:01/95 to $12: 31: 95$

Page 1

\begin{tabular}{|c|c|c|c|c|c|c|c|c|c|}
\hline $\begin{array}{l}\text { Warning Levels: } \\
\text { Unioff Laces }\end{array}$ & $\begin{array}{l}9.0 E-12 \\
5 r-90 \\
\mu C 1 / / \text { III }\end{array}$ & $\begin{array}{l}\text { 3. } 0 \mathrm{E} \cdot \mathrm{H1} \\
\mathrm{Ru} \cdot 106 \\
a[1: \mathrm{in}]\end{array}$ & 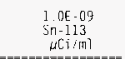 & $\begin{array}{c}\text { 1. DE }-09 \\
5 \mathrm{~b} \cdot 125 \\
\text { WCi, i mi }\end{array}$ & $\begin{array}{l}2.0 E \cdot 10 \\
C s \cdot 134 \\
\text { if } 13 . \mathrm{ml}\end{array}$ & $\begin{array}{l}4.0<10 \\
C 5.137 \\
10 i, i m]\end{array}$ & 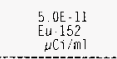 & $\begin{array}{l}5.0 E-11 \\
\text { Eu. } 154 \\
\mu C i ; m]\end{array}$ & $\begin{array}{l}\text { 3. DE- } 10 \\
\text { Eu-155 } \\
\mu[1 / \mathrm{TII}\end{array}$ \\
\hline $\begin{array}{l}12 / 27 / 94-03 / 28 / 95 \\
03: 28: 95-06 / 20 / 95 \\
06 / 20 / 95 \text { 09:12:95 }\end{array}$ & $\begin{array}{l}7.3 \mathrm{E}-14 \pm 10 \\
3.9 \mathrm{E}-14 \pm 15 \mathrm{E} \\
4.4 \mathrm{E}-14 \pm 108\end{array}$ & $\begin{aligned}-1.8 \mathrm{E}-16 & \pm 450^{\circ} \\
-3.0 \mathrm{E} \cdot 16 & \pm 420^{\circ} \\
1.5 \mathrm{E} .15 & \pm 100^{\circ}\end{aligned}$ & $\begin{array}{l}-9 \cdot 0 E-17 \pm 120 \% \\
-1.3 E-16 \pm 130 \% \\
5.6 E \cdot 19 \pm 9999 t\end{array}$ & $\begin{aligned} &-2.6 \mathrm{E}-16 \pm 100 \% \\
& 1.3 \mathrm{E}-16 \pm 280 \\
& 1.2 \mathrm{E}-16 \pm 290 \%\end{aligned}$ & $\begin{array}{l}\text { B. } 3 \mathrm{E} 1 / \pm 120 \% \\
5 \mathrm{E} \cdot 17 \pm 910^{\circ} \\
1.5 \mathrm{E} \cdot 18 \pm 8000^{\circ}\end{array}$ & $\begin{array}{ll}1.6 \mathrm{E} \cdot 14 \pm 20 \\
1.9 \mathrm{E} 14 \pm 20 \% \\
2.4 \mathrm{E} \cdot 14 \pm & \pm 8 \%\end{array}$ & $\begin{array}{l}1.4 \mathrm{E}-16 \pm 125 \mathrm{a} \\
3 \cdot 7 \mathrm{E}-17 \pm 330 t \\
3 \cdot 9 \mathrm{E}-17 \pm 330 t\end{array}$ & $\begin{array}{l}3.1 \mathrm{E}-16 \pm 850 \\
35 \mathrm{E}-16 \pm 120 \mathrm{t} \\
6 \mathrm{E} \cdot 1 / \pm 600 \%\end{array}$ & $\begin{array}{r}1.0 \mathrm{E}-15 \pm 240 \% \\
-1.0 \mathrm{E}-16 \pm 360 \% \\
2.9 \mathrm{E}-16 \pm 100 \%\end{array}$ \\
\hline
\end{tabular}

06:21:96 11:21

291-T-1: 221 I CAVYCN \& CF 15 (X785)

$01 / 01 / 95 \quad 10 \quad 12 / 31: 95$

Page 2

\begin{tabular}{|c|c|c|c|c|c|c|c|}
\hline $\begin{array}{l}\text { Warning Levels: } \\
\text { On/Off Dates }\end{array}$ & $\begin{array}{l}3.0 \mathrm{E}-14 \\
\mathrm{Pu}-238 \\
\mu \mathrm{CT} / \mathrm{m1}\end{array}$ & 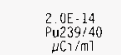 & $\begin{array}{l}2.0 E \cdot 14 \\
\sin 241 \\
\omega C \mathrm{C} / \mathrm{m} 1\end{array}$ & $\begin{array}{l}\text { Col ect } \\
\text { Tinie } \\
\text { Hours }\end{array}$ & $\begin{array}{l}\text { Sample } \\
\text { Flowprate } \\
\text { CFM }\end{array}$ & $\begin{array}{l}\text { Sample } \\
\text { Woilune } \\
\text { tt3 }\end{array}$ & $\begin{array}{l}\text { Stack } \\
\text { Flowkate } \\
\text { DFM }\end{array}$ \\
\hline $\begin{array}{l}2 / 27 / 94-03 / 28 / 95 \\
3 / 28,95-06 / 20 / 95 \\
6 / 20 / 95-09 / 12 / 95\end{array}$ & 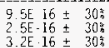 & 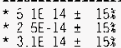 & $\begin{array}{l}2.6 \mathrm{EE} .15 \pm 15 \% \\
2.0 \mathrm{E}=15 \pm 20 \% \\
2.7 \mathrm{E} \cdot 15 \pm 20 \%\end{array}$ & $\begin{array}{l}2112.1 \\
1511.0 \\
1874.9\end{array}$ & & $\begin{array}{l}220900 \\
150000 \\
210000\end{array}$ & \\
\hline
\end{tabular}

5YHBC KEY: * Concentration exceeds Warning Level 5 H Hít exceeds Warning Levei. 


\title{
WHC-SD-EN-TI-308 Rev.0
}

WHC ENVIRONMENTAL RELEASE SUMMARY

\author{
296-T-7: 2706-T stack (T154) \\ $01 / 01 / 95$ through $12 / 31 / 95$ \\ Total System Flow: $2.3 \mathrm{E}+10$ Liters $=8.0 \mathrm{E}+08$ Cubic Ft
}

The following factor(s) has (or have) been applied to the radionuclide concentrations used in the calculation of this release to account for sampling filter efficiency, line and bend loss, and humidity. The concentrations vary inversely with the size of the factor $(s)$.

$$
\text { Particulates: } \quad 0.70
$$

\begin{tabular}{cc} 
Radionuclide & Release, Ci \\
\hline Total $\alpha$ & $5.0 \mathrm{E}-09$ \\
Total $B$ & $4.6 \mathrm{E}-08$
\end{tabular}

$\begin{array}{cc}\begin{array}{c}\text { Concentrations, } \mu \mathrm{Ci} / \mathrm{ml} \\ \text { Average }\end{array} & \text { Peak } \\ ------- & ----- \\ \text { 1. } 5 \mathrm{E}-16 & 4.2 \mathrm{E}-15 \\ 1.4 \mathrm{E}-15 & 1.5 \mathrm{E}-14\end{array}$


WHC-SD-EN-TI-308 Rev.0
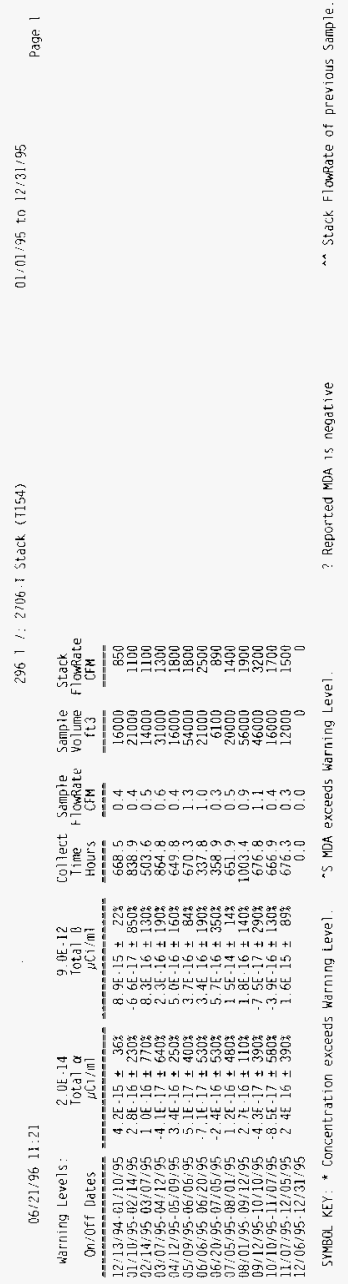


\section{WHC-SD-EN-TI-308 Rev.0}

WHC ENVIRONMENTAL RELEASE SUMMARY

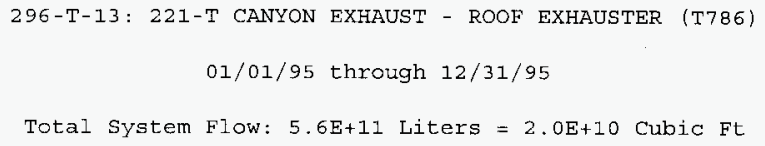

$$
\text { Particulates: } \quad 0.73
$$

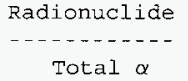

$\begin{array}{cc}\text { Concentrations, } \mu \mathrm{Ci} / \mathrm{ml} \\ \text { Average } & \text { Peak } \\ ------ & ----- \\ 1.5 \mathrm{E}-15 & 1.2 \mathrm{E}-14 \\ 1.2 \mathrm{E}-14 & \text { 8. } 5 \mathrm{E}-14\end{array}$




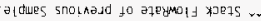

$26 \mathrm{p}$

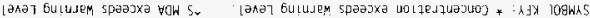

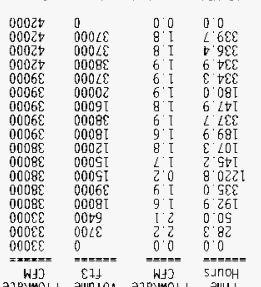

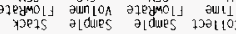

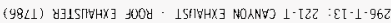

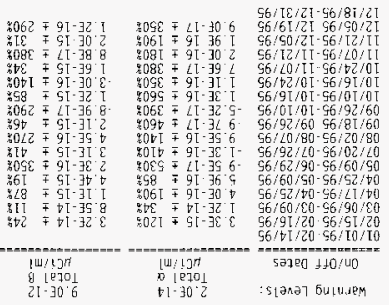

LZ: II $96,[7 / 90$ 
WHC-SD-EN-TI-308 Rev.0

2.5.3 TRUSAF 


\section{WHC-SD-EN-TI-308 Rev.0}

WHC ENVIRONMENTAL RELEASE SUMMARY

$06 / 15 / 96$

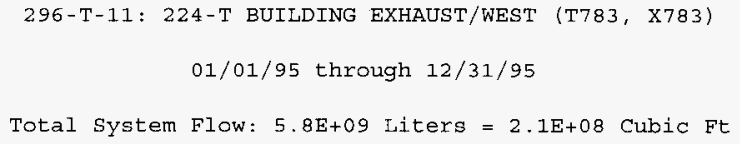
to account for sampling filter efficiency, line and bend loss, and humidity. The concentrations vary inversely with the size of the factor(s).

\begin{tabular}{|c|c|c|c|}
\hline \multirow[b]{3}{*}{ Radionuclide } & culates: & \multicolumn{2}{|l|}{0.73} \\
\hline & & Concentrat & is, $\mu \mathrm{Ci} / \mathrm{ml}$ \\
\hline & Release, Ci & Average & Peak \\
\hline---------- & $---\cdots----$ & ------- & ------ \\
\hline Total $\alpha$ & $7.8 \mathrm{E}-10$ & $9.8 E-17$ & 1. $2 E-16$ \\
\hline Total B & $3.8 E-09$ & $4.7 E-16$ & 5. $8 E-16$ \\
\hline
\end{tabular}


WHC-SD-EN-TI-308 Rev.0
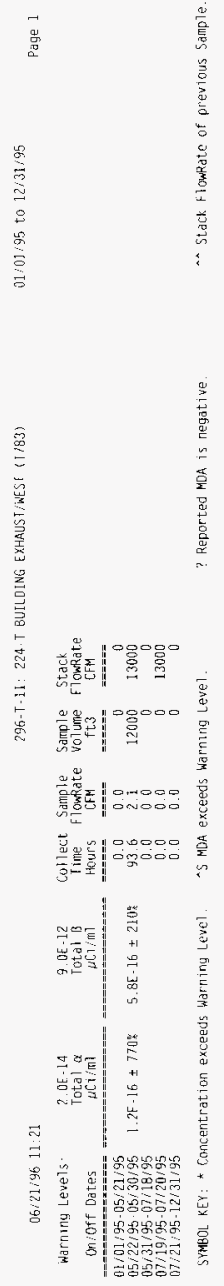


\section{WHC-SD-EN-TI-308 Rev.0}

WHC ENVIRONMENTAL RELEASE SUMMARY

$$
\text { 296-T-12: 224-T BUILDING EXHAUST/EAST (T784) }
$$

$01 / 01 / 95$ through $12 / 31 / 95$

Total system Flow: $1.4 E+11$ Liters $=5.0 E+09$ Cubic Ft

The following factor(s) has (or have) been applied to the radionuclide concentrations used in the calculation of this release to account for sampling filter efficiency, line and bend loss, and humidity. The concentrations vary inversely with the size of the factor (s).

\begin{tabular}{cccc}
\multicolumn{2}{c}{ Particulates: } & 0.73 \\
Radionuclide & Release, Ci & Concentrations, $\mu \mathrm{Ci} / \mathrm{ml}$ \\
Average & Peak \\
Total $\alpha$ & $1.2 \mathrm{E}-07$ & $-1 .-15-16$ & $3.5 \mathrm{E}-15$ \\
Total $\mathrm{B}$ & $7.9 \mathrm{E}-07$ & $4.1 \mathrm{E}-15$ & $1.0 \mathrm{E}-14$
\end{tabular}


06/21/96 11:21

\begin{tabular}{|c|c|c|c|c|c|c|}
\hline $\begin{array}{l}\text { Warning Levels: } \\
\text { Unioff Oaces }\end{array}$ & 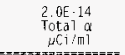 & $\begin{array}{l}9 \text {. BE- } 12 \\
\text { Total B } \\
\text { pCisimi } \\
=-==-=\end{array}$ & $\begin{array}{l}\text { Collect } \\
\text { T1me } \\
\text { Hours } \\
=-=-5\end{array}$ & $\begin{array}{l}\text { Sample } \\
\text { Flowkate } \\
\text { CFM } \\
\text { Fe }\end{array}$ & $\begin{array}{c}\text { Sample } \\
\text { volume } \\
\text { ft3 } \\
=-==\end{array}$ & $\begin{array}{l}\text { Stack } \\
\text { Flowrate } \\
\text { CFH } \\
\text { - }\end{array}$ \\
\hline 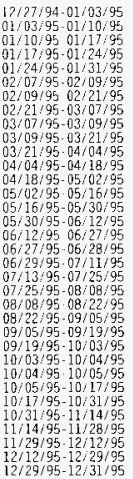 & 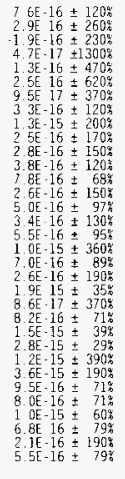 & 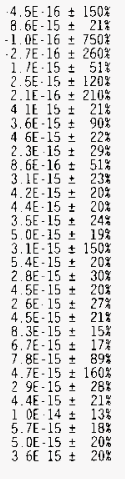 & $\begin{array}{l}144.9 \\
162.0 \\
168.9 \\
166.9 \\
166.1 \\
48.1 \\
287.2 \\
333.9 \\
47.1 \\
279.3 \\
339.7 \\
334.1 \\
339.1 \\
332.6 \\
330.5 \\
311.7 \\
335.9 \\
29.7 \\
288.0 \\
269.9 \\
335.3 \\
334.4 \\
336.8 \\
335.4 \\
337.2 \\
23.7 \\
18.8 \\
283.6 \\
335.7 \\
336.8 \\
33.8 \\
312.1 \\
42.2 \\
0.0\end{array}$ & $\begin{array}{l}2.7 \\
1.9 \\
1.9 \\
1.9 \\
1.9 \\
1.8 \\
1.9 \\
1.9 \\
1.9 \\
1.9 \\
1.9 \\
2.0 \\
2.0 \\
1.9 \\
1.8 \\
1.7 \\
1.7 \\
1.7 \\
1.8 \\
1.7 \\
1.7 \\
1.8 \\
1.7 \\
1.8 \\
1.8 \\
1.8 \\
1.8 \\
1.9 \\
1.8 \\
0.8\end{array}$ & $\begin{array}{l}19000 \\
18000 \\
19000 \\
19000 \\
19000 \\
5200 \\
32000 \\
37000 \\
5300 \\
310000 \\
37000 \\
39000 \\
41000 \\
40000 \\
38000 \\
33000 \\
35000 \\
3100 \\
3000 \\
28000 \\
35000 \\
35000 \\
35000 \\
35000 \\
36000 \\
2500 \\
2000 \\
31000 \\
36000 \\
37000 \\
36000 \\
33000 \\
45000 \\
450\end{array}$ & $\begin{array}{l}10000 \\
10000 \\
10000 \\
10000 \\
10000 \\
10000 \\
10000 \\
10000 \\
10000 \\
10900 \\
10000 \\
10000 \\
10000 \\
100000 \\
109000 \\
10000 \\
10000 \\
10000 \\
100000 \\
9109 \\
9100 \\
9100 \\
9100 \\
9100 \\
9100 \\
9100 \\
9100 \\
9109 \\
9100 \\
9109 \\
9100 \\
9100 \\
9100 \\
9100\end{array}$ \\
\hline
\end{tabular}

01/01:95 to $12 / 31: 95$

Page 1 
WHC-SD-EN-TI-308 Rev.0

This page intentionally left blank. 
WHC-SD-EN-TI-308 Rev.0

2.5.4 West Tank Farms 


\title{
WHC-SD-EN-TI-308 Rev.0
}

WHC ENVIRONMENTAL RELEASE SUMMARY

\author{
296-P-22: ALL SY ANNULI (W191) \\ $01 / 01 / 95$ through $12 / 31 / 95$
}

Total System Flow: $1.8 E+10$ Liters $=6.2 \mathrm{E}+08$ Cubic Ft

The following factor(s) has (or have) been applied to the radionuclide concentrations used in the calculation of this release to account for sampling filter efficiency, line and bend loss, and humidity. The concentrations vary inversely with the size of the factor (s).

$$
\text { Particulates: } \quad 0.80
$$

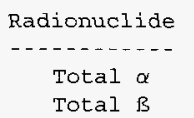

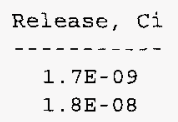

\begin{tabular}{cc}
$\begin{array}{c}\text { Concentrations, } \mu \mathrm{Ci} / \mathrm{ml} \\
\text { Average }\end{array}$ & Peak \\
\hline $7.8 \mathrm{E}-17$ & $5.2 \mathrm{E}-16$ \\
8. $3 \mathrm{E}-16$ & $4.1 \mathrm{E}-15$
\end{tabular}


? Reported MDA is negative.
06/21/96 11:22

\begin{tabular}{|c|c|c|c|c|c|c|}
\hline $\begin{array}{l}\text { Warning Levels: } \\
\text { On/oft Dates }\end{array}$ & $\begin{array}{l}2.0 E \cdot 14 \\
\text { Tota7 } \\
\mu \mathrm{Ci} / \mathrm{m}\end{array}$ & $\begin{array}{l}9.0 \mathrm{E}-12 \\
\text { Total } \\
\mu \mathrm{C} \mathrm{T} / \mathrm{mi}\end{array}$ & $\begin{array}{c}\text { Collect. } \\
\text { Time } \\
\text { Hours }\end{array}$ & $\begin{array}{l}\text { Sample } \\
\text { Flowkate. } \\
\text { CFM }\end{array}$ & $\begin{array}{l}\text { Sample } \\
\text { volume } \\
\mathrm{ft3}\end{array}$ & $\begin{array}{l}\text { Stack } \\
\text { Flowreate } \\
\text { CFM }\end{array}$ \\
\hline 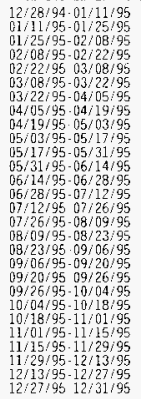 & 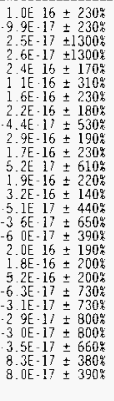 & 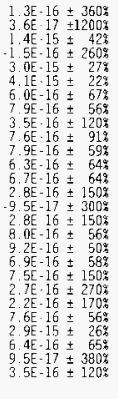 & $\begin{array}{l}337.2 \\
331.0 \\
331.9 \\
340.5 \\
332.0 \\
334.6 \\
333.8 \\
335.4 \\
340.9 \\
207.7 \\
335.0 \\
335.9 \\
335.8 \\
33.1 \\
334.6 \\
331.1 \\
335.8 \\
336.0 \\
349.2 \\
147.9 \\
183.8 \\
299.1 \\
333.4 \\
332.0 \\
336.3 \\
339.8 \\
332.1 \\
0.0\end{array}$ & $\begin{array}{l}1.7 \\
1.8 \\
1.8 \\
1.7 \\
1.7 \\
1.8 \\
1.7 \\
1.9 \\
1.9 \\
1.7 \\
1.7 \\
1.7 \\
1.7 \\
1.7 \\
1.7 \\
1.8 \\
1.4 \\
1.6 \\
2.0 \\
1.7 \\
1.7 \\
1.7 \\
1.8 \\
0.0\end{array}$ & $\begin{array}{l}34000 \\
360000 \\
360000 \\
34000 \\
33000 \\
33000 \\
36000 \\
34000 \\
35000 \\
24000 \\
34000 \\
35000 \\
33000 \\
34000 \\
34000 \\
34000 \\
35000 \\
35000 \\
36000 \\
12000 \\
17000 \\
36000 \\
35000 \\
35000 \\
35000 \\
34000 \\
36000 \\
0\end{array}$ & $\begin{array}{l}1200 \\
1200 \\
1200 \\
12200 \\
1200 \\
1200 \\
1200 \\
1200 \\
1200 \\
1200 \\
1200 \\
1200 \\
1200 \\
1200 \\
1200 \\
1200 \\
1200 \\
1200 \\
1200 \\
850 \\
1200 \\
1000 \\
1200 \\
1200 \\
1200 \\
1200 \\
1200 \\
1200\end{array}$ \\
\hline
\end{tabular}

296-P-22. ALL 5Y ANNLLI] (WI91

45 MDA exceeds warning level.
[1/:9]/95 to 12/31/95 
WHC-SD-EN-TI-308 Rev.0

ENVIRONMENTAL RELEASE SUMMARY REPORT

$08 / 02 / 96$

296-P-23\& 296-P-28: EXHAUSTERS FOR ALI SY TANKS (W190, W195)

$01 / 01 / 95$ through $12 / 31 / 95$

Total System Flow: $1.8 \mathrm{E}+10$ Liters $=6.4 \mathrm{E}+08$ Cubic Ft

Radionuclide

-.-....-.

${ }^{90} \mathrm{Sr}$

${ }^{106} \mathrm{Ru}$

${ }^{113} \mathrm{Sr}$

$125 \mathrm{Sb}$

${ }^{134} \mathrm{Cs}$

${ }^{137} \mathrm{Cs}$

$152 \mathrm{Eu}$

${ }^{154} \mathrm{Eu}$

${ }^{155} \mathrm{Eu}$

${ }^{239} \mathrm{Pu}$

$239 / 240 \mathrm{Pu}$

${ }^{241} \mathrm{Am}$

Total $\alpha$

Total is

Halflife
29.12 Years
368 Days
115 Days
2.8 Years
2.1 Years
30 Years
13.3 Years
8.8 Years
4.96 Years
87.8 Years
24,131 Years*
432 Years
n/a
n/a

Release, $\mathrm{Ci}$

-..........

1. $3 E-07$

4. 0E-09

6. $0 \mathrm{E}-10$

3. $4 \mathrm{E}-09$

4. $\mathrm{OE}-10$

4. OE- 06

1. $7 E-09$

ND

2. $4 \mathrm{E}-09$

3. $9 \mathrm{E}-10$

1. $7 \mathrm{E}-09$

3. $9 E-09$

1. $1 \mathrm{E}-08$

$2.9 E-06$

* The halflife shown for Total Uranium is the halflife of ${ }^{238} \mathrm{U}$. The halflife shown for mixed $239 / 240 \mathrm{Pu}$ is the halflife of ${ }^{239} \mathrm{Pu}$. 
$06 / 21 / 96 \quad 1]: ? ?$

245 P 23. EKHALISTER FOR A.L SY TANKS (W190)

\begin{tabular}{|c|c|c|c|c|c|c|}
\hline $0 f f$ da & $\begin{array}{l}\text { Total } \\
\mu C 7 ? n\end{array}$ & $\begin{array}{c}\text { Total } \\
\mu[\mathbf{i} / \mathrm{m}\end{array}$ & $\begin{array}{l}\text { Collect } \\
\text { Time } \\
\text { Hours }\end{array}$ & $\begin{array}{l}\text { Samgle } \\
\text { ilowide } \\
C F M\end{array}$ & $\begin{array}{l}\text { Sample } \\
\text { volune } \\
\text { ft3 } 3\end{array}$ & $\begin{array}{l}\text { Stack } \\
\text { Flowkate } \\
\text { CFM }\end{array}$ \\
\hline 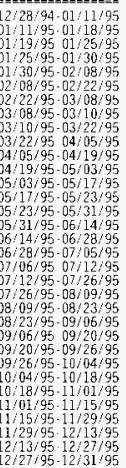 & 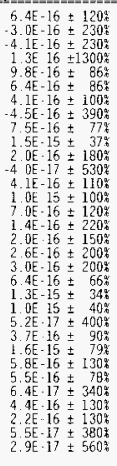 & 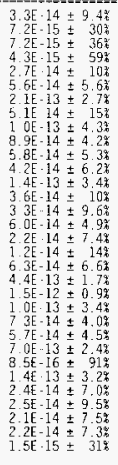 & $\begin{array}{l}143.5 \\
119.8 \\
212.0 \\
336.7 \\
335.5 \\
45.0 \\
283.6 \\
333.9 \\
335.4 \\
340.8 \\
331.4 \\
143.7 \\
186.9 \\
330.7 \\
335.8 \\
165.9 \\
145.2 \\
310.6 \\
331.7 \\
335.8 \\
336.0 \\
331.5 \\
102.0 \\
143.4 \\
287.4 \\
333.7 \\
178.5 \\
336.3 \\
326.6 \\
309.8 \\
0.1\end{array}$ & $\begin{array}{l}\frac{2}{2} \\
\frac{2}{2} \\
2 \\
2 \\
2 \\
2 \\
2 \\
2 \\
2 \\
2 \\
2 \\
\frac{2}{2}\end{array}$ & $\begin{array}{l}330 \\
590\end{array}$ & $\begin{array}{r}100 \\
1100 \\
820 \\
1100\end{array}$ \\
\hline
\end{tabular}

SYMBOL KEY: * Concentration exceeds Warming Level.
01/01/95 to 12/31/95

Page 1

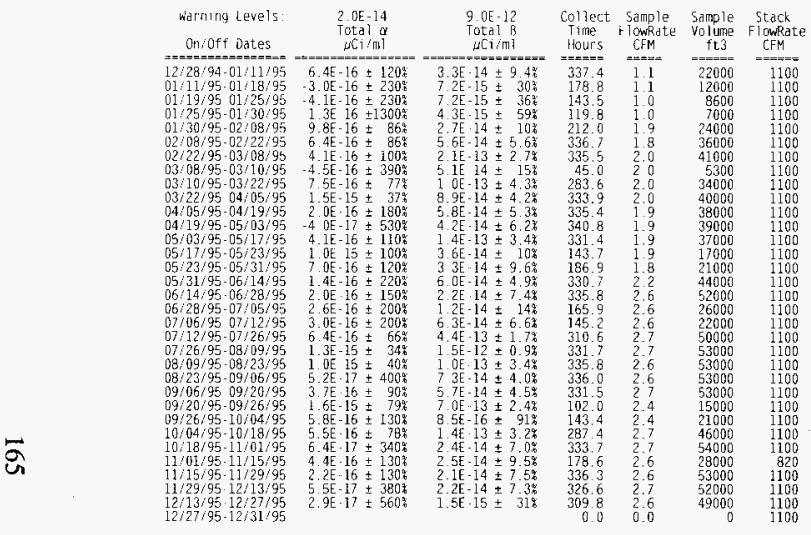

? Reported MDA is negative

^ Stack Flcwrate of previous Sample 


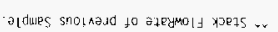

2 aced $96 / 16 / 210796 / 10 / 10$ anizegau SL YoW pat koday:

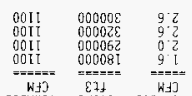

Wf 3 cts $=$

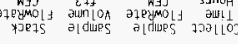

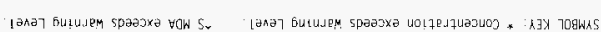

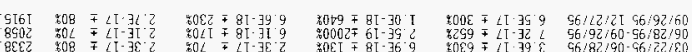

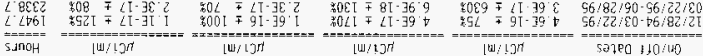

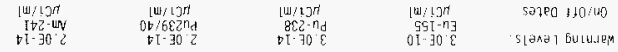
$22:$ TL $95 ;$ [2: $: 90$

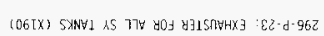

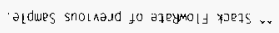

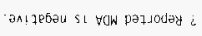

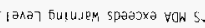

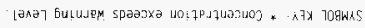

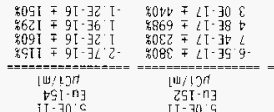

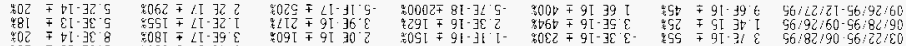

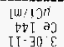

int

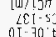

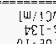

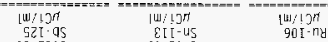

I $3 \sin ^{-4}$

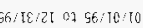

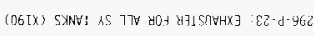


06:21:96 11:22

\begin{tabular}{|c|c|c|c|c|c|c|}
\hline 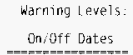 & $\begin{array}{l}2.0 E-14 \\
\text { Totat } \alpha \\
\text { Tolimi } \\
=====\end{array}$ & 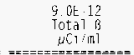 & 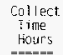 & 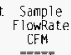 & $\begin{array}{l}\text { Sample } \\
\text { volume } \\
\text { ft } \\
===\end{array}$ & $\begin{array}{l}\text { Stack } \\
\text { Flowiate } \\
\text { CFM }\end{array}$ \\
\hline 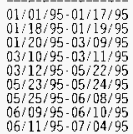 & $7 . ? F \cdot 15 \pm 230.5$ & $U E 1 \zeta \pm 6 B^{\circ}$ & $\begin{aligned} r==0 \\
0.0 \\
15.7 \\
0.0 \\
0.0 \\
0.0 \\
0.0 \\
0.0\end{aligned}$ & & $\begin{array}{r}1600 \\
0\end{array}$ & $\begin{array}{r}890 \\
0 \\
890 \\
0 \\
890\end{array}$ \\
\hline & $14 \mathrm{E} \cdot 15 \pm 450 \%$ & $1.8 E \cdot 15 \pm 400$ & & & $1700^{\circ}$ & 890 \\
\hline & 8 IE $16=4$ & 4. $3 E \cdot 15 \pm 1508$ & & & 2200 & 5 \\
\hline & $2.1 E-16 \pm 560$ & $1.8 E-15 \pm 130$ & 56 & 2 & 6700 & 45 \\
\hline & $6.5 \bar{f} \cdot 16$ & 2. $6 \in 15 \pm 110$ & 9 & & 5300 & 4 \\
\hline & 8 ot $17 \pm 8.05 \%$ & $1.1 E \cdot 15 \pm 110^{\circ}$ & เ28. & 0.0 & $13000^{\circ}$ & $\begin{array}{l}15 \\
38\end{array}$ \\
\hline o & & & & & 100 & \\
\hline & & & & & & \\
\hline & & & & & & \\
\hline
\end{tabular}

SYMBOL KEY: * ConLentration exceeds warning Leve1.

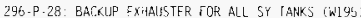

^S MDA exceeds warning Level
? Reported MDA is regative
21:01:95 to 12:31:95

Page 1 
$06: 21 \% 96011: 22$

296́ P 20: RACKUP EYI HALSTER FOR ALL SY MANKS $(Y 19 b)$

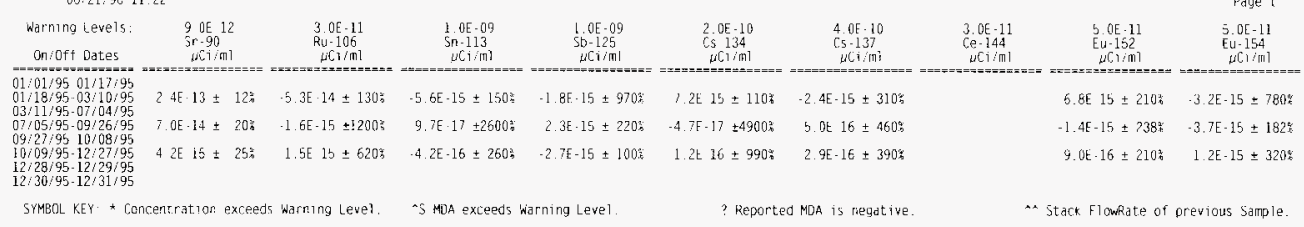

06. 2.1.96 11.22

\begin{tabular}{|c|c|c|c|c|}
\hline $\begin{array}{l}\text { Wiaming Levels: } \\
\text { On/Off Dates }\end{array}$ & $\begin{array}{l}3.0 E] U \\
E U-155 \\
\mu C i / \pi I]\end{array}$ & 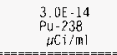 & 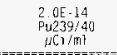 & $\begin{array}{l}2.0 \mathrm{E}-14 \\
\mathrm{Am}-24 \mathrm{I} \\
\mu \mathrm{Ci} / \mathrm{III}]\end{array}$ \\
\hline $\begin{array}{l}1 / 01995-01 / 17 / 95 \\
1 / 18 / 9503 / 1095\end{array}$ & $6.6 \mathrm{t} \cdot 10 \pm 300 *$ & $15 \mathrm{t} \cdot 16 \pm 640 \%$ & 3. $9 E-16 \pm 330 \%$ & 1. $1 \mathrm{E}-14 \pm$ \\
\hline $03 / 05 / 95.09 / 26 / 95$ & 3 JE. $15 \pm 136 \%$ & $2.1 E-16 \pm 270 \%$ & 3. $1 E-16 \pm 210 \%$ & 6. $4 E-16 \pm$ \\
\hline $\begin{array}{l}19 / 27 / 95-10 / 08 / 95 \\
10 ! 09 / 95-12 / 27 / 95 \\
12 / 28 / 95 \cdot 12 ! 29 / 95 \\
12 / 30 / 95-12 / 31 / 95\end{array}$ & $5.4 E-17 \pm 4700 \%$ & $1 \cdot 1[-17=680 \%$ & $3.8 E-16 \pm 60 \%$ & 4. OE $16 \pm$ \\
\hline
\end{tabular}

SYMBOL KEY: * Concentration exceeds warning Level. IS MDA exceeds Warnisig Level.
796-P-28: BACKUF EXHAUSTLR FCR ALL SY TANKS ( $K 195)$

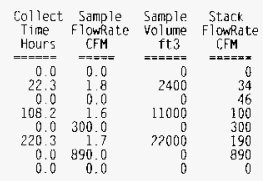

? Reported MDA is negative.
01/01/95 to 12:31:95

Page 2 


\section{WHC-SD-EN-TI-308 Rev.0}

WHC ENVIRONMENTAL RELEASE SUMMARY

296-S-15: EXHAUSTER FOR ALL SX TANKS (W111, X111)

$01 / 01 / 95$ through $12 / 31 / 95$

Total system Flow: $8.7 E+10$ Liters $=3.1 E+09$ Cubic Ft

The following factor(s) has (or have) been applied to the radionuclide concentrations used in the calculation of this release to account for sampling filter efficiency, line and bend loss, and humidity. The concentrations vary inversely with the size of the factor $(s)$.

\begin{tabular}{|c|c|c|c|}
\hline \multirow[b]{2}{*}{ Radionuclide } & culates: & \multicolumn{2}{|l|}{0.84} \\
\hline & Release, Ci & $\begin{array}{c}\text { Concentrati } \\
\text { Average }\end{array}$ & 1s, $\mu \mathrm{Ci} / \mathrm{ml}$ \\
\hline$\ldots \ldots+-\ldots+\cdots$ & ----------- & ------- & $\cdots \cdots--$ \\
\hline${ }^{90} \mathrm{Sr}$ & $6.6 \mathrm{E}-08$ & $6.4 \mathrm{E}-16$ & I. $2 \mathrm{E}-15$ \\
\hline${ }^{106} \mathrm{Ru}$ & $\mathrm{ND}$ & $-2.6 E-17$ & 3. $3 E-16$ \\
\hline${ }^{113} \mathrm{Sn}$ & $2.1 \mathrm{E}-09$ & 2. $0 \mathrm{E}-17$ & 5. $1 E-17$ \\
\hline${ }^{125} \mathrm{Sb}$ & $\mathrm{ND}$ & $-4.8 E-17$ & $5.3 E-17$ \\
\hline${ }^{134} \mathrm{Cs}$ & 1. $7 E-09$ & $1.6 \mathrm{E}-17$ & 1. $1 E-16$ \\
\hline${ }^{137} \mathrm{Cs}$ & $1.1 E-07$ & 1. $1 E-15$ & 3. $2 E-15$ \\
\hline${ }^{152} \mathrm{Eu}$ & $2.7 E-09$ & $2.6 \mathrm{E}-17$ & 1. $2 E-16$ \\
\hline${ }^{154} \mathrm{Eu}$ & $5.4 E-09$ & $5.3 \mathrm{E}-17$ & $2.4 \mathrm{E}-16$ \\
\hline${ }^{155} \mathrm{Eu}$ & $6.5 E-09$ & $6.3 E-17$ & 1. $3 E-16$ \\
\hline${ }^{238} \mathrm{Pu}$ & $5.4 F-10$ & $5.3 E-18$ & 1. $3 E-17$ \\
\hline $239 / 240 \mathrm{Pu}$ & $1.8 E-09$ & $1.8 E-17$ & 3. $5 E-I 7$ \\
\hline${ }^{241} \mathrm{Am}$ & $2.7 E-09$ & $2.6 \mathrm{E}-17$ & 5. $3 E-17$ \\
\hline Total $\alpha$ & $2.7 E-08$ & $2.7 \mathrm{E}-16$ & $8.5 E-15$ \\
\hline Total is & $4.1 \mathrm{E}-07$ & $4.0 \mathrm{E}-15$ & $4.1 \mathrm{E}-14$ \\
\hline
\end{tabular}


(16/21/46 $11 \cdot 21$

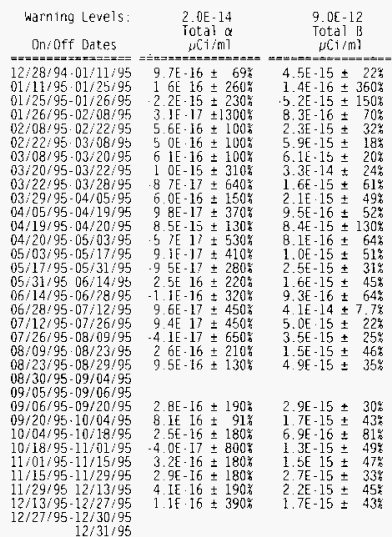

SYMBOL KEY : * Concentration exceeds Warning Leve
296-5.15. FXIAALSTER FOH ALL SX TANKS W'W111

\begin{tabular}{|c|c|c|c|}
\hline $\begin{array}{l}\text { Collect. } \\
\text { Time } \\
\text { Hour's }\end{array}$ & $\begin{array}{c}\text { Sample } \\
\text { Flowkate } \\
\text { CFM }\end{array}$ & $\begin{array}{l}\text { Sainple } \\
\text { volume } \\
\mathrm{ft} .3\end{array}$ & $\begin{array}{l}\text { Stack } \\
\text { Flowkat } \\
C F M\end{array}$ \\
\hline & 1 & 33000 & 5000 \\
\hline $\begin{array}{l}338.8 \\
16.8\end{array}$ & & 32000 & \\
\hline $\begin{array}{r}10.0 \\
309.4\end{array}$ & & 29000 & \\
\hline 3367 & 1.6 & 33000 & 6000 \\
\hline $\begin{array}{l}3.51 .0 \\
281.6\end{array}$ & & $\begin{array}{l}33000 \\
27000\end{array}$ & $\begin{array}{l}6 \\
6\end{array}$ \\
\hline 353 & . & 36600 & 60 \\
\hline $\begin{array}{l}163.7 \\
163 .\end{array}$ & .7 & $\begin{array}{l}15000 \\
17000\end{array}$ & 5 \\
\hline $\begin{array}{l}335.5 \\
145\end{array}$ & 6 & $\begin{array}{l}32000 \\
1400\end{array}$ & 5000 \\
\hline 300 & 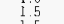 & $\begin{array}{l}27000 \\
27000\end{array}$ & 6000 \\
\hline $\begin{array}{l}328.3 \\
375.2\end{array}$ & 5 & $\begin{array}{r}29000 \\
30000\end{array}$ & $\begin{array}{l}6000 \\
6000\end{array}$ \\
\hline $\begin{array}{l}326 . \\
331\end{array}$ & 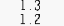 & $\begin{array}{l}250000 \\
24000\end{array}$ & $\begin{array}{l}5000 \\
5600\end{array}$ \\
\hline $\begin{array}{l}332.8 \\
335.0\end{array}$ & 3 & $\begin{array}{r}25000 \\
25000\end{array}$ & 6000 \\
\hline $\begin{array}{l}3.32 \\
3.32\end{array}$ & & 30000 & $\begin{array}{l}b \\
6 \\
c\end{array}$ \\
\hline $\begin{array}{l}346.7 \\
146\end{array}$ & & 12000 & $\begin{array}{l}6000 \\
6000\end{array}$ \\
\hline 0 & & & 0 \\
\hline 3300 & 3 & 25000 & 6000 \\
\hline $\begin{array}{l}328 \\
340\end{array}$ & ${ }^{2}$ & $\begin{array}{l}25000 \\
31000\end{array}$ & $\begin{array}{l}50 \\
60\end{array}$ \\
\hline 329 & & 25000 & 6000 \\
\hline 33 & & 24000 & 6000 \\
\hline $\begin{array}{l}335 \\
225\end{array}$ & & $\begin{array}{l}26000 \\
17000\end{array}$ & 6 \\
\hline 332. & 1.3 & 26000 & 6000 \\
\hline 0 & 0.0 & & 6000 \\
\hline & & & \\
\hline
\end{tabular}

$01 / 01 / 95$ to $12: 31 / 95$

Fage ] 
$06: 21: 9611: 21$

256 5. 15: [XHAUSTER FOR AII 5X TANKS (X111)

$01 / 01 / 95$ to $12 / 31: 95$

\begin{tabular}{|c|c|c|c|c|c|c|c|c|c|}
\hline $\begin{array}{l}\text { Warnong tevels: } \\
\text { Urisuft Dates } \\
=============\end{array}$ & 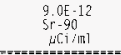 & $\begin{array}{l}3.0 E 11 \\
\text { Ru-106 } \\
\mu[1,1 \mathrm{~m} 1\end{array}$ & 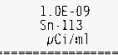 & 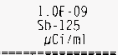 & $\begin{array}{l}2.0 \mathrm{E}-10 \\
05-134 \\
0[1 / 191\end{array}$ & $\begin{array}{c}4.0 E-10 \\
C 5-137 \\
40 \mathrm{i} ; \mathrm{ml}\end{array}$ & $\begin{array}{l}\text { 3. } 0 \mathrm{E}-11 \\
\mathrm{Ce}-14.4 \\
\mu \mathrm{C}, i \mathrm{ml}\end{array}$ & $\begin{array}{l}\text { 5. OE - } 11 \\
\text { Eu } 152 \\
\mu C i \text { (tnl }\end{array}$ & $\begin{array}{l}\text { 5. } 0[-11 \\
\text { Eu- }-154 \\
\mu[1] \text { ? }[1]\end{array}$ \\
\hline 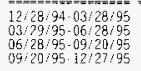 & $\begin{array}{l}\text { 3. 3E } 36 \pm 12 \% \\
36 E-16 \pm 75 \% \\
12 E-15 \pm 30 \% \\
676-16 \pm 25 \%\end{array}$ & $\begin{array}{r}3.3 \mathrm{E}-16 \pm 2757 \\
2.3 \mathrm{16} \pm 390 \\
9.3 \mathrm{E}-18 \pm 9999 \% \\
-5.7 \mathrm{E}-16 \pm 210 \%\end{array}$ & 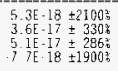 & $\begin{array}{r}-7.6 \mathrm{E}-17 \pm 3150 \\
5.3 \mathrm{~F}-17 \pm 4305 \\
-1.3 \mathrm{E}-16 \pm 2225 \\
4.7 \mathrm{E}-17 \pm 590 \%\end{array}$ & 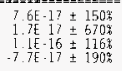 & 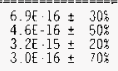 & & $\begin{array}{l}1.2 \mathrm{E}-16 \pm 160 \% \\
-6.7 \mathrm{E}-17 \pm 260 t \\
6.1 \mathrm{E} 17 \pm 348 \% \\
1.0 \mathrm{E} .16 \pm 230 \%\end{array}$ & $\begin{array}{l}-53 E-17 \pm 550 \% \\
3.0 E-17 \pm 1100 \% \\
2.4 E-16 \pm 160 \% \\
20 E-17 \pm 2300 \%\end{array}$ \\
\hline
\end{tabular}

$06: 21: 96 \quad 11 \cdot 21$

296-S.15: EXHAUSTER FOR ALI SX TANKS (X:11)

$01 / 01 / 95$ to $12 / 31 / 95$

Page 2

\begin{tabular}{|c|c|c|c|c|c|c|c|c|}
\hline $\begin{array}{l}\text { Warming levels. } \\
\text { OniOff Dates }\end{array}$ & $\begin{array}{l}3.0 \mathrm{E} \cdot 10 \\
\mathrm{Eu}-155 \\
\mu \mathrm{C} i \mathrm{i} \text { ? }\end{array}$ & 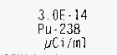 & $\begin{array}{l}2.0 \mathrm{E} \cdot 14 \\
\mathrm{P} u 239 / 40 \\
\mu C \mathrm{C} / \mathrm{ml}\end{array}$ & $\begin{array}{l}2.0 \mathrm{E}-14 \\
\text { An }-241 \\
\text { iC } \mathrm{Ci} / \mathrm{m} 1\end{array}$ & $\begin{array}{l}\text { Collect } \\
\text { Time } \\
\text { Hour's }\end{array}$ & $\begin{array}{l}\text { Sample } \\
\text { FlowRate } \\
\text { CFFf }\end{array}$ & $\begin{array}{l}\text { Sample } \\
\text { volume } \\
\mathrm{ft3} 3\end{array}$ & $\begin{array}{c}\text { stack } \\
\text { Flowhate } \\
\text { CFAt }\end{array}$ \\
\hline $\begin{array}{l}12 / 28 / 9403 / 28 / 95 \\
03 / 29 / 95-06 / 92 / 95 \\
06 / 28 / 95-09 / 20 / 95 \\
09 / 20 / 95-12 / 27 / 95\end{array}$ & 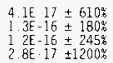 & $\begin{array}{l}\text { 3.7F }-18 \pm 460 \% \\
6.18-19 \pm 400 \% \\
2.7 \mathrm{E}-18 \pm 460 \% \\
13 \mathrm{E} 17 \pm 170 \%\end{array}$ & $\begin{array}{l}\text { 1.3E } 17 \pm 110 \% \\
6.4 \mathrm{E}-18 \pm 270 \\
3.5 \mathrm{E}-17 \pm 80 \%\end{array}$ & $\begin{array}{r}-9 \mathrm{E} \cdot 18 \pm 100 \% \\
5.3 \mathrm{E} .18 \pm 340^{\circ} \\
4.17 \pm 80^{\circ} \\
5.3 \mathrm{E} \cdot 17 \pm 60^{\circ}\end{array}$ & $\begin{array}{l}1777.8 \\
2135.6 \\
1825.5 \\
2220.1\end{array}$ & & $\begin{array}{l}170000 \\
190000 \\
140000 \\
170000\end{array}$ & $\begin{array}{l}6000 \\
5600 \\
5600 \\
5800\end{array}$ \\
\hline
\end{tabular}

SYMBOL KEY. * Concentration exceeds Warning Level. 5 MDA exceeds Wärnhg Level. ? Reported MDA is negative.

a stack Flowkate of previous sample. 


\title{
WHC-SD-EN-TI-308 Rev.0
}

WHC ENVIRONMENTAL RELEASE SUMMARY

\author{
296-S-18: 242-S BUILDING EXHAUST (W096, X096) \\ $01 / 01 / 95$ through $12 / 31 / 95$ \\ Total System Flow: $2.2 \mathrm{E}+11$ Liters $=7.9 \mathrm{E}+09$ Cubic Ft
}

The following factor(s) has (or have) been applied to the radionuclide concentrations used in the calculation of this release to account for sampling filter efficiency, line and bend loss, and humidity. The concentrations vary inversely with the size of the factor $(s)$.

$$
\text { Particulates: } \quad 0.69
$$

$\begin{array}{cc}\text { Radionuclide } & \text { Release, Ci } \\ \text { Total } \alpha & 3.8 \mathrm{E}-08 \\ \text { Total is } & 6.4 \mathrm{E}-07\end{array}$

$\begin{array}{cc}\begin{array}{c}\text { Concentrations, } \mu \mathrm{Ci} / \mathrm{ml} \\ \text { Average }\end{array} & \text { Peak } \\ ------ & ----- \\ \text { 1. } 2 \mathrm{E}-16 & 5.5 \mathrm{E}-16 \\ \text { 2. } 0 \mathrm{E}-15 & 1.9 \mathrm{E}-14\end{array}$


06:?1:96 $11 \cdot 7:$

\begin{tabular}{|c|c|c|c|c|c|c|}
\hline $\begin{array}{l}\text { Wornng Levels: } \\
\text { On/off bates }\end{array}$ & 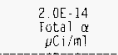 & 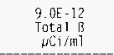 & $\begin{array}{l}\text { Collect } \\
\text { Time } \\
\text { Hours }\end{array}$ & $\begin{array}{l}\text { Sample } \\
\text { Flowinte } \\
\text { CFM }\end{array}$ & $\begin{array}{l}\text { Sample } \\
\text { Volune } \\
\mathrm{ft3} 3\end{array}$ & $\begin{array}{l}\text { Stack } \\
\text { Flowrate } \\
\text { CFM }\end{array}$ \\
\hline \multirow{2}{*}{ 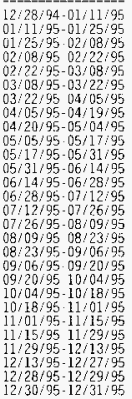 } & $\begin{array}{l}? .5 \mathrm{E}-17 \pm 1300^{\circ} \\
1.6 \mathrm{E}-16 \pm 260^{\circ} \\
1.6 \mathrm{E} \cdot 16 \pm 260^{\circ} \\
2.8 \mathrm{E} 17 \pm 1300^{\circ} \\
2.6 \mathrm{E} \cdot 16 \pm 170^{\circ} \\
2.6 \mathrm{E} \cdot 16 \pm 1709 \\
8.5 \mathrm{E} 1 / \pm 280^{\circ} \\
1.6 \mathrm{E} \cdot 16 \pm 370^{\circ}\end{array}$ & 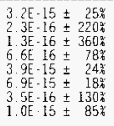 & $\begin{array}{l}337.3 \\
336.5 \\
331.9 \\
330.6 \\
335.3 \\
734.0 \\
335.3 \\
189.1\end{array}$ & $\begin{array}{l}18 \\
17 \\
17 \\
16 \\
15 \\
16\end{array}$ & $\begin{array}{l}35000 \\
34000 \\
33000 \\
31000 \\
30000 \\
31000 \\
34000 \\
19000\end{array}$ & \\
\hline & 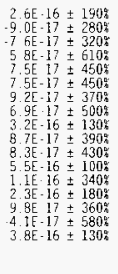 & 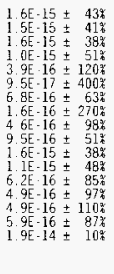 & $\begin{array}{r}275.8 \\
319.7 \\
332.5 \\
332.2 \\
317.2 \\
331.1 \\
330.2 \\
319.5 \\
336.0 \\
340.6 \\
330.8 \\
340.0 \\
318.5 \\
332.0 \\
327.3 \\
318.2 \\
332.9 \\
0.0 \\
0.0\end{array}$ & 0.0 & $\begin{array}{r}26000 \\
32000 \\
33000 \\
32000 \\
32000 \\
32000 \\
33000 \\
33000 \\
35000 \\
32000 \\
32000 \\
30000 \\
32000 \\
34000 \\
32000 \\
32000 \\
31000 \\
0 \\
0\end{array}$ & $\begin{array}{l}23000 \\
23000 \\
23000 \\
23000 \\
10000 \\
10000 \\
7200 \\
19000 \\
10000 \\
10000 \\
10000 \\
10000 \\
10000 \\
10000 \\
10000 \\
10060 \\
10000 \\
10000\end{array}$ \\
\hline
\end{tabular}




\section{WHC-SD-EN-TI-308 Rev.0}

$06 / 15 / 96$

WHC ENVIRONMENTAL RELEASE SUMMARY

296-S-22: 244-S SALTWELL RECEIVER \& ANNUL. (W880, X880)

** 01/01/95 through $12 / 31 / 95$

Total System Flow: $1.7 \mathrm{E}+09$ Liters $=5.9 \mathrm{E}+07$ Cubic $\mathrm{Ft}$

The following factor(s) has (or have) been applied to the radionuclide concentrations used in the calculation of this release to account for sampling filter efficiency, line and bend loss, and humidity. The concentrations vary inversely with the size of the factor (s).

$$
\text { Particulates: } \quad 0.72
$$

\begin{tabular}{|c|c|c|c|}
\hline Radionuclide & Release, $\mathrm{Ci}$ & $\begin{array}{c}\text { oncentrati } \\
\text { Average }\end{array}$ & ns, $\begin{array}{c}\mu \mathrm{Ci} / \mathrm{ml} \\
\text { Peak }\end{array}$ \\
\hline----------- & - - - - - & -------- & ------ \\
\hline${ }^{90} \mathrm{Sr}$ & $2.1 \mathrm{E}-08$ & $9.0 \mathrm{E}-15$ & 1. $6 \mathrm{E}-14$ \\
\hline${ }^{106} \mathrm{Ru}$ & $\mathrm{ND}$ & $-4.0 E-16$ & $5.9 E-16$ \\
\hline${ }^{113} \mathrm{Sn}$ & $\mathrm{ND}$ & $-8.6 E-17$ & 2. $1 \mathrm{E}-17$ \\
\hline${ }^{125} \mathrm{Sb}$ & 3. $2 \mathrm{E}-10$ & 1. $4 E-16$ & $1.5 \mathrm{E}-16$ \\
\hline${ }^{134} \mathrm{Cs}$ & $\mathrm{ND}$ & $-4.6 E-17$ & $-2.8 E-17$ \\
\hline${ }^{137} \mathrm{Cs}$ & $4.2 \mathrm{E}-10$ & 1. $8 \mathrm{E}-16$ & $1.8 \mathrm{E}-16$ \\
\hline${ }^{152} \mathrm{Eu}$ & $\mathrm{ND}$ & $-4.3 E-16$ & $-1.1 \mathrm{E}-16$ \\
\hline${ }^{154} \mathrm{Eu}$ & ND & $-1 \cdot 2 E-16$ & $8.6 \mathrm{E}-18$ \\
\hline${ }^{155} \mathrm{Eu}$ & $5.0 \mathrm{E}-10$ & $2.2 E-16$ & $4.6 \mathrm{E}-16$ \\
\hline${ }^{238} \mathrm{Pu}$ & ND & $-1.8 E-18$ & $6.8 \mathrm{E}-18$ \\
\hline $239 / 240 \mathrm{Pu}$ & $1.4 \mathrm{E}-10$ & $6.0 E-17$ & $5.9 \mathrm{E}-17$ \\
\hline $241 \mathrm{Am}$ & 4. $1 E-10$ & 1. $8 E-16$ & 1. $9 E-16$ \\
\hline Total $\alpha$ & 6. $1 E-10$ & $2.6 \mathrm{E}-16$ & 1. $8 E-15$ \\
\hline Total B & $8.4 E-09$ & $3.6 \mathrm{E}-15$ & $1.9 \mathrm{E}-14$ \\
\hline
\end{tabular}


$06 / 21 / 96 \quad 11: 22$

\begin{tabular}{|c|c|c|}
\hline $\begin{array}{l}\text { Warming Levels: } \\
\text { On/off Dates }\end{array}$ & $\begin{array}{c}\text { Total } \alpha \\
\nu \subset i / m 1\end{array}$ & 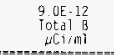 \\
\hline \multirow{5}{*}{ 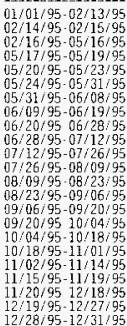 } & $1.8 \mathrm{E}-15 \pm 150_{\mathrm{e}}^{4}$ & $3.5 \mathrm{~F}-15 \pm 71$ \\
\hline & $\begin{array}{l}\text { 6. } 8 \mathrm{E}, 1 / \pm \\
40 \mathrm{E}-16 \pm\end{array}$ & $\begin{array}{l}\text { 1. } 5 \mathrm{E}-15 \pm 51 \mathrm{z} \\
3.3 \mathrm{E}-15 \pm 28 *\end{array}$ \\
\hline & 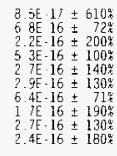 & $\begin{array}{l}5.7 \mathrm{E}-16 \pm 120 \% \\
1.8 \mathrm{E} \cdot 15 \pm 31 \% \\
3.6 \mathrm{E}-15 \pm 24 \% \\
3.2 \mathrm{E}-16 \pm 150 \% \\
1.4 \mathrm{E}-15 \pm 36 \% \\
16 \mathrm{E}-15 \pm 34 \% \\
1.9 \mathrm{E}-15 \pm 29 \% \\
1.8 \mathrm{E}-15 \pm 31 \% \\
9.2 \mathrm{E}-16 \pm 43 \% \\
6.2 \mathrm{E}-15 \pm 18 \%\end{array}$ \\
\hline & $-1.5 \mathrm{E}-16 \pm 660^{\circ}$ & $2.3 \mathrm{E}-15 \pm 91 \%$ \\
\hline & 0 E: $6 \pm 560$ & $1.9 \mathrm{E}-14 \pm 33 \%$ \\
\hline
\end{tabular}

SYMBCL KEY: * Concentration, exceeds warning Level.
296-5 22: 244-5 SAL TWELI RECFJWFR \& ANAL, IW580)

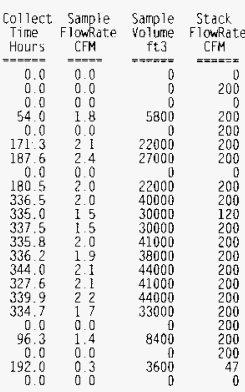

-5 moa exceeds warning Level.
011:01/95 to 12:31/95

Page 1

\section{$\overline{4}$}

? Rerorted MDA is negative. 


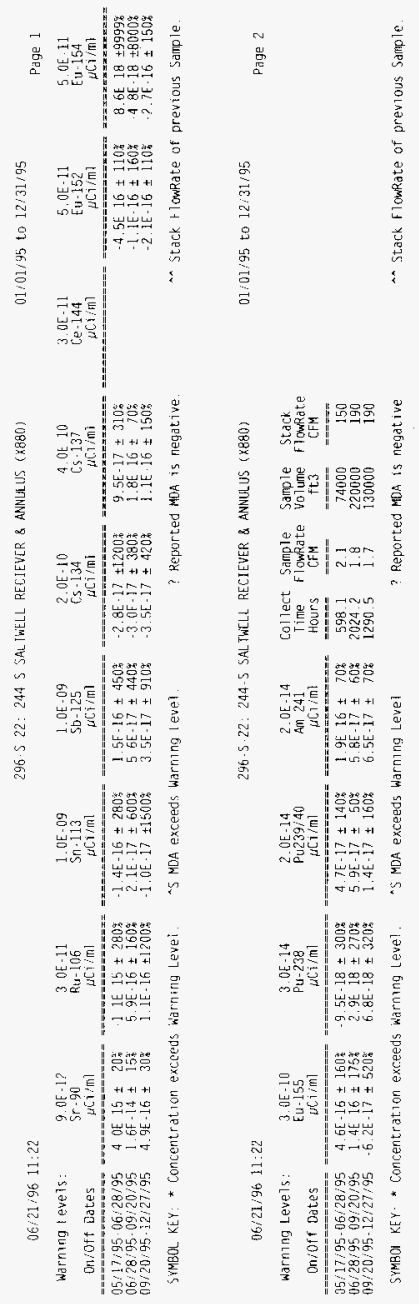




\section{WHC-SD-EN-TI-308 Rev.0}

WHC ENVIRONMENTAL RELEASE SUMMARY

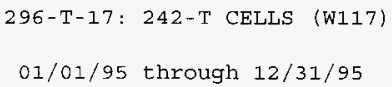
to account for sampling filter efficiency, line and bend loss, and humidity. The concentrations vary inversely with the size of the factor(s).

$$
\text { Particulates: } \quad 0.81
$$

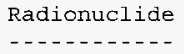

Total $\alpha$

Total $\beta$

Release, $\mathrm{Ci}$
2.2E-09
$1.8 \mathrm{E}-08$

Release, $\mathrm{Ci}$

2. $2 E-09$

$1.8 E-08$

$\begin{array}{cc}\text { Concentrations, } \mu \mathrm{Ci} / \mathrm{ml} \\ \text { Average } & \text { Peak } \\ & ------ \\ 5.0 \mathrm{E}-17 & 8.4 \mathrm{E}-16 \\ 5.1 \mathrm{E}-16 & 4.2 \mathrm{E}-15\end{array}$


06:21:96́ 11:21

\begin{tabular}{|c|c|c|c|c|c|c|}
\hline $\begin{array}{l}\text { Warning Leve?s: } \\
\text { Onoff Dates }\end{array}$ & $\begin{array}{l}2.0 \mathrm{E}-1 \mathrm{a} \\
\text { Total } \alpha \\
\mu \mathrm{cis} / \mathrm{ml}\end{array}$ & 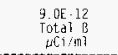 & $\begin{array}{l}\text { Collect. } \\
\text { Time } \\
\text { Hours }\end{array}$ & $\begin{array}{l}\text { Samole } \\
\text { Flowiate } \\
\text { CFM }\end{array}$ & $\begin{array}{l}\text { Sarnole } \\
\text { volume } \\
\text { vt } \mathbf{t}^{3}\end{array}$ & $\begin{array}{l}\text { Stack } \\
\text { FlowPd } \\
C F M\end{array}$ \\
\hline 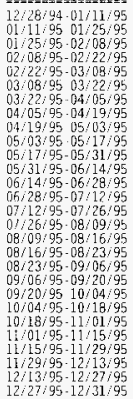 & 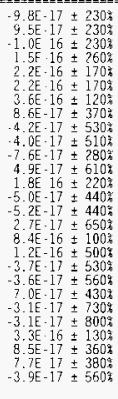 & 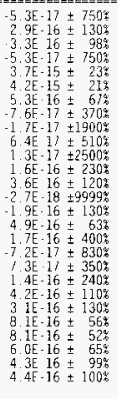 & $\begin{array}{l}339.0 \\
336.0 \\
332.4 \\
333.7 \\
336.7 \\
335.2 \\
337.0 \\
336.3 \\
334.3 \\
340.1 \\
332.4 \\
336.3 \\
334.4 \\
336.3 \\
332.0 \\
333.9 \\
165.1 \\
164.0 \\
336.4 \\
335.6 \\
333.2 \\
336.3 \\
304.2 \\
335.4 \\
335.6 \\
336.7 \\
335.0 \\
0.0\end{array}$ & 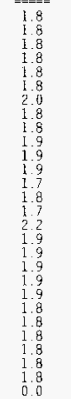 & $\begin{array}{l}=== \\
36000 \\
37000 \\
35000 \\
36000 \\
360000 \\
36000 \\
40000 \\
36000 \\
37000 \\
38000 \\
38000 \\
38000 \\
35000 \\
35000 \\
34000 \\
45000 \\
18000 \\
19000 \\
39000 \\
39000 \\
37000 \\
36000 \\
33000 \\
37000 \\
37000 \\
37000 \\
36000\end{array}$ & \\
\hline
\end{tabular}

C1/01/95 to $12: 31 / 95$

Page 1 


\section{WHC-SD-EN-TI-308 Rev.0}

$06 / 15 / 96$

WHC ENVIRONMENTAI, RELEASE SUMMARY

296-T-18: 244-TX SALT WELL RECEIVER \& ANN. (W882, X882)

$01 / 01 / 95$ through $12 / 31 / 95$

Total System Flow: $5.4 \mathrm{E}+09$ Liters $=1.9 \mathrm{E}+08$ Cubic Ft

The following factor(s) has (or have) been applied to the radionuclide concentrations used in the calculation of this release to account for sampling filter efficiency, line and bend loss, and humidity. The concentrations vary inversely with the size of the factor (s).

\begin{tabular}{|c|c|c|c|}
\hline \multirow[b]{2}{*}{ Radionuclide } & culates: & \multicolumn{2}{|l|}{0.70} \\
\hline & Release, $\mathrm{Ci}$ & $\begin{array}{c}\text { Concentrati } \\
\text { Average }\end{array}$ & $\begin{array}{l}\text { ns, } \mu \mathrm{Ci} / \mathrm{ml} \\
\text { Peak }\end{array}$ \\
\hline---------- & --------- & ------- & $-\ldots-\ldots$ \\
\hline${ }^{90} \mathrm{Sr}$ & 1. $0 \mathrm{E}-10$ & 1. $3 E-17$ & 2. $7 \mathrm{E}-16$ \\
\hline${ }^{106} \mathrm{Ru}$ & $\mathrm{ND}$ & $-1.9 E-16$ & $9.4 E-I 7$ \\
\hline${ }^{113} \mathrm{Sn}$ & ND & $-1.1 E-17$ & 3. OE-17 \\
\hline${ }^{125} \mathrm{Sb}$ & $\mathrm{ND}$ & $-6.7 E-17$ & 1. $7 \mathrm{E}-17$ \\
\hline${ }^{134} \mathrm{Cs}$ & 2. $3 E-10$ & 3. $O E-17$ & 1. $1 \mathrm{E}-16$ \\
\hline${ }^{137} \mathrm{Cs}$ & $2.6 \mathrm{E}-08$ & $3.4 \mathrm{E}-15$ & $7.2 \mathrm{E}-15$ \\
\hline${ }^{152} \mathrm{Eu}$ & ND & $-3.6 E-17$ & $-2.4 \mathrm{E}-17$ \\
\hline${ }^{154} \mathrm{Eu}$ & $\mathrm{ND}$ & $-5.5 E-17$ & 4. $9 E-17$ \\
\hline${ }^{155} \mathrm{Eu}$ & $6.2 E-10$ & $7.9 \mathrm{E}-17$ & $1.9 E-16$ \\
\hline${ }^{238} \mathrm{Pu}$ & 2.1E-11 & $2.7 E-18$ & $1.1 E-17$ \\
\hline $239 / 240 \mathrm{Pu}$ & 1. $0 \mathrm{E}-09$ & 1. $3 E-16$ & $3.8 \mathrm{E}-16$ \\
\hline${ }^{241} \mathrm{Am}$ & $5.8 \mathrm{E}-10$ & $7.4 E-17$ & 8. $9 \mathrm{E}-17$ \\
\hline Total $\alpha$ & 1. $8 \mathrm{E}-09$ & 2. $3 E-16$ & $7.7 E-16$ \\
\hline Total is & $2.5 E-08$ & $3.3 E-15$ & 2. $8 E-14$ \\
\hline
\end{tabular}


06:21/95 11:22

\begin{tabular}{|c|c|c|c|c|c|c|}
\hline $\begin{array}{l}\text { Warnifig Levels: } \\
\text { On: aft Dates }\end{array}$ & $\begin{array}{c}2.0 E-14 \\
\text { Total } \\
\text { TCi/mi }\end{array}$ & $\begin{array}{c}9.0 E \cdot 12 \\
\text { Total } 13 \\
\mu C 1 / m i\end{array}$ & $\begin{array}{l}\text { Collect } \\
\text { Time } \\
\text { Hours }\end{array}$ & $\begin{array}{l}\text { Sample } \\
\text { Flowiate } \\
\text { Cim }\end{array}$ & $\begin{array}{l}\text { Sample } \\
\text { volume } \\
\mathrm{ft} 3\end{array}$ & $\begin{array}{c}\text { Stack } \\
\text { Flowkate } \\
\text { CFM }\end{array}$ \\
\hline \multirow{2}{*}{ 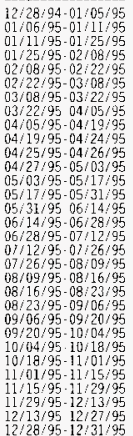 } & 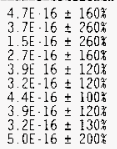 & 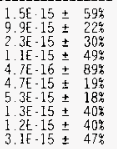 & $\begin{array}{l}199.3 \\
115.3 \\
335.9 \\
332.5 \\
334.5 \\
335.1 \\
335.1 \\
337.2 \\
336.3 \\
119.1\end{array}$ & $\begin{array}{l}1.7 \\
2.1 \\
1.8 \\
1.8 \\
1.8 \\
1.9 \\
1.9 \\
1.9 \\
1.9\end{array}$ & $\begin{array}{l}21000 \\
14000 \\
36000 \\
36000 \\
37000 \\
33000 \\
38000 \\
37000 \\
38000 \\
13000\end{array}$ & $\begin{array}{l}370 \\
370 \\
370 \\
370 \\
370 \\
370 \\
370 \\
370 \\
370 \\
370\end{array}$ \\
\hline & 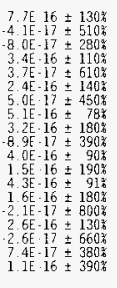 & 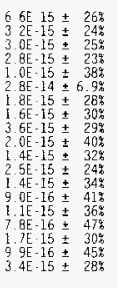 & $\begin{array}{l}0.0 \\
142.4 \\
340.5 \\
332.5 \\
336.1 \\
334.5 \\
336.2 \\
337.8 \\
334.0 \\
164.8 \\
171.0 \\
336.3 \\
335.5 \\
333.3 \\
336.3 \\
335.1 \\
336.6 \\
335.6 \\
337.0 \\
335.5 \\
0.0\end{array}$ & $\begin{array}{l}2.3 \\
2.4 \\
2.3 \\
2.3 \\
2.4 \\
2.4 \\
2.3 \\
2.3 \\
1.9 \\
1.3 \\
0.0\end{array}$ & $\begin{array}{l}15000 \\
37000 \\
36000 \\
44000 \\
49000 \\
46600 \\
49000 \\
47000 \\
23000 \\
24000 \\
48000 \\
46000 \\
47000 \\
49000 \\
49000 \\
47000 \\
46000 \\
38000 \\
27000\end{array}$ & $\begin{array}{l}370 \\
370 \\
370 \\
370 \\
370 \\
370 \\
370 \\
370 \\
370 \\
370 \\
370 \\
370 \\
370 \\
370 \\
370 \\
370 \\
370 \\
370\end{array}$ \\
\hline
\end{tabular}

01:01:95 to 12/31/95

Page i 


\section{$06: 21 / 9611: 22$}

296-T-18: 244 TX SALI WELL RECEIVIF \& ANNULUIS (X8B2)

\begin{tabular}{|c|c|c|c|c|c|c|c|c|c|}
\hline $\begin{array}{l}\text { Warning Levels: } \\
\text { Oniofi vates }\end{array}$ & 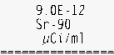 & $\begin{array}{l}3.0 E-11 \\
\text { Ru-106 } \\
\text { uciolimI }\end{array}$ & 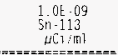 & 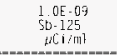 & 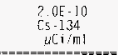 & $\begin{array}{l}4.0 E 10 \\
C 5-137 \\
\mu[i / \pi\end{array}$ & 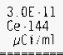 & 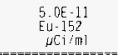 & 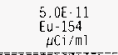 \\
\hline $\begin{array}{l}12 / 28 / 94 \cdot 03 / 22 / 95 \\
03 / 22 / 95.06 / 28 / 95 \\
06 / 28 / 95.09 / 20 / 95 \\
09 / 20 / 95 \quad 12 / 27 / 95\end{array}$ & $\begin{array}{l}-3.2 E-16 \pm 100 \% \\
-5 E-17 \pm 400 \% \\
1.1 E 16 \pm 16 \% \\
\text { 2. } 7 E \cdot 16 \pm 80 \%\end{array}$ & $\begin{aligned}-6.2 E-16 \pm 1700 \\
-3.3 E-16 \pm 1900 \\
9.4 E-17 \pm 5278 \\
5.6 E 11 \pm 9703\end{aligned}$ & $\begin{array}{r}5.6 \mathrm{E} .17 \pm 480 \% \\
2.2 \mathrm{E}-17 \pm 400 \% \\
3.0 \mathrm{E}-17 \pm 2640 \\
-4.3 \mathrm{E}-17 \pm 160 \%\end{array}$ & $\begin{aligned} &-2.2 \mathrm{E}-16 \pm 100 \% \\
&-1.8 \mathrm{E}-17 \pm 8708 \\
& 1.7 \mathrm{E} 17 \pm 10006 \\
&-5.7 \mathrm{E}-17 \pm 250 \%\end{aligned}$ & 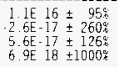 & $\begin{array}{l}2.9 \mathrm{E}-15 \pm 208 \\
2.9 \mathrm{e}=15 \pm 20 \% \\
7.2 \mathrm{f}-15 \pm 19 \% \\
9.4 \mathrm{E}-16 \pm 25 \%\end{array}$ & & 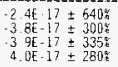 & $\begin{array}{r}-6 \mathrm{E}-16 \pm 1500 \\
-1.0 \mathrm{E}-16 \pm 220 \% \\
-2 \cdot 2 \mathrm{E}-17 \pm 9440 \\
4.9 \mathrm{E}-17 \pm 400^{a}\end{array}$ \\
\hline
\end{tabular}

SYMBC KEY: * Concentration exceeds warning Level a 5 MOA exceeds Warning Level. ? Reported MDA is negative.

296-T-I8: 244.TX SALI WELL RECELYER \& ANNULUS ( $\times 882)$

$166: 21 / 9611: 22$

\begin{tabular}{|c|c|c|c|c|c|c|c|c|}
\hline $\begin{array}{l}\text { Warning Levels: } \\
\text { On:Of } f \text { Dates }\end{array}$ & $\begin{array}{l}3.0 \mathrm{E}-10 \\
\mathrm{Eu}-155 \\
\mathrm{j} \cdot \mathrm{Ci} / \mathrm{m}\end{array}$ & $\begin{array}{l}\text { 3. OE- } 14 \\
\begin{array}{c}\mathrm{Pu}-238 \\
j \mathrm{C} i \text { iml }\end{array}\end{array}$ & $\begin{array}{l}2.0 \mathrm{CE}-14 \\
\mathrm{~F}=239 / 40 \\
\mu \mathrm{CO} i \mathrm{ml}\end{array}$ & 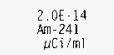 & $\begin{array}{l}\text { Collect } \\
\text { Tine } \\
\text { Hours }\end{array}$ & $\begin{array}{l}\text { Simple } \\
\text { Fiowride } \\
\text { CFM }\end{array}$ & $\begin{array}{l}\text { 5ample } \\
\text { yolume } \\
\text { yt3 }\end{array}$ & $\begin{array}{l}\text { Stack } \\
\text { Flawkate } \\
\text { CFM }\end{array}$ \\
\hline $\begin{array}{l}12 / 28 / 94-03 / 22 / 95 \\
03 / 22 / 95.06 / 28 / 95 \\
06 / 28 / 95.09 / 20 / 95 \\
09 / 20 / 95-12 / 27 / 95\end{array}$ & $\begin{array}{l}1.9 \mathrm{E}-16 \pm 115 \% \\
4.1 \mathrm{E}-17 \pm 380^{\circ} \\
3.4 \mathrm{E}-19 \pm 9999^{\circ} \\
9.2 \mathrm{E}-17 \pm 180^{\circ}\end{array}$ & 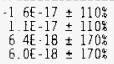 & $\begin{array}{l}3.8 \mathrm{E} \cdot 16 \pm 759 \\
4.9 \mathrm{E}-17 \pm 60 \% \\
6.5 \mathrm{E}-17 \pm 50 \% \\
7.1 \mathrm{E}-17 \pm 40 \%\end{array}$ & 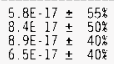 & $\begin{array}{l}1989.7 \\
2278.5 \\
2015.6 \\
2349.4\end{array}$ & $\begin{array}{l}1.8 \\
2.0 \\
2.3 \\
2.2\end{array}$ & $\begin{array}{l}2200040 \\
270000 \\
280000 \\
300000\end{array}$ & $\begin{array}{l}370 \\
370 \\
370 \\
360\end{array}$ \\
\hline
\end{tabular}

SYMBO KEY: * Concentration exceeds warning lever

-S MDA exceeds harning Leyel.

? Reported MDA is negative.
Q1:01/95 to 12/31/95

Page 2

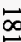




\section{WHC-SD-EN-TI-308 Rev.0}

296-W-3: 213-W WASTE COMPACTOR (W003)

$01 / 01 / 95$ through $12 / 31 / 95$

Total System Flow: $3.1 \mathrm{E}+10$ Liters $=1.1 \mathrm{E}+09$ Cubic Ft.

The following factor(s) has (or have) been applied to the radionuclide concentrations used in the calculation of this release to account for sampling filter efficiency, line and bend loss, and humidity. The concentrations vary inversely with the size of the factor (s).

$$
\text { Particulates: } \quad 0.73
$$

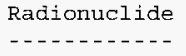

Total $\alpha$

Total $B$

Release, Ci
$1.4 \mathrm{E}-08$
$5.9 \mathrm{E}-08$

Release, $\mathrm{Ci}$

$1.4 \mathrm{E}-08$

$\begin{array}{cc}\text { Concentrations, } \mu \mathrm{Ci} / \mathrm{ml} \\ \text { Average } & \text { Peak } \\ ------ & ------ \\ \text { 3.2E-16 } & \text { 1. OE-15 } \\ \text { 1.4E-15 } & \text { 5. } 6 \mathrm{E}-15\end{array}$


$06: 21: 96: 1: 21$

\begin{tabular}{|c|c|c|c|c|c|c|}
\hline $\begin{array}{l}\text { Warning Levels } \\
\text { On; Off Dates }\end{array}$ & 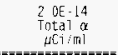 & $\begin{array}{l}9.0 \mathrm{E}-12 \\
\text { Total } \\
\mu \mathrm{Ci} / \mathrm{mi}\end{array}$ & $\begin{array}{l}\text { Collect } \\
\text { Time } \\
\text { Thours }\end{array}$ & $\begin{array}{c}\text { Sarple } \\
\text { Hrowfiate } \\
C F M\end{array}$ & & $\begin{array}{l}\text { Stack } \\
\text { Flowfat } \\
\text { CTM }\end{array}$ \\
\hline $\begin{array}{l}104 / 95-10 / 18 / 9 \\
18 / 9511 / 01 / 9 \\
10195-11 / 15 / 9 \\
15 / 95-11 / 29 / 9 \\
29995-12 / 13 / 9 \\
13 / 95-12 / 27 / 9 \\
127 / 95-12 / 31 / 9\end{array}$ & 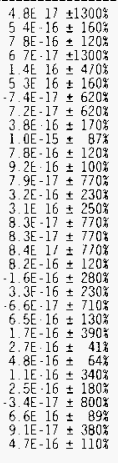 & 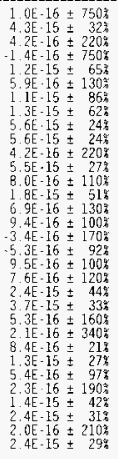 & $\begin{array}{l}168 \\
167 \\
16 \\
107 \\
168 \\
170 \\
165 \\
167 \\
167 \\
168 \\
167 \\
168 \\
164 \\
193 \\
168 \\
166 \\
167 \\
168 \\
168 \\
164 \\
164\end{array}$ & & $\begin{array}{l}180 \\
1 B 0 \\
180 \\
130 \\
180 \\
180 \\
180 \\
210 \\
210 \\
180 \\
180\end{array}$ & \\
\hline
\end{tabular}

SYMBOL KEY: * Concentration exceeds harnifig teve

S MDA exceeds Warning Level. 
WHC-SD-EN-TI-308 Rev.0

This page intentionally left blank. 
WHC-SD-EN-TI-308 Rev.0

2.5.5 WSCF 


\section{WHC-SD-EN-TI-308 Rev.0}

WHC ENVIRONMENTAL RELEASE SUMMARY

695-W-1: WSCF LABORATORY MAIN STACK EXHAUST (WO10)

$01 / 01 / 95$ through $12 / 31 / 95$

Total system Flow: $7.4 \mathrm{E}+11$ Liters $=2.6 \mathrm{E}+10$ Cubic Ft

The following factor(s) has (or have) been applied to the radionuclide concentrations used in the calculation of this release to account for sampling filter efficiency, line and bend loss, and humidity. The concentrations vary inversely with the size of the factor (s).

$$
\text { Particulates: } \quad 0.73
$$

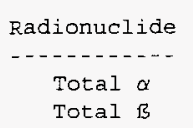

Radionuclide
Release, Ci -.-.......
9. $4 E-08$
5. OE-07

$\begin{array}{cc}\begin{array}{c}\text { Concentrations, } \mu \mathrm{Ci} / \mathrm{ml} \\ \text { Average }\end{array} & \text { peak } \\ - & ------ \\ 9.2 \mathrm{E}-17 & 7.2 \mathrm{E}-16 \\ 4.9 \mathrm{E}-16 & 4.8 \mathrm{E}-15\end{array}$




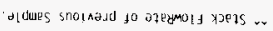

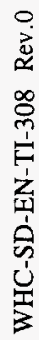

-aAllebau si Wh pay unday :

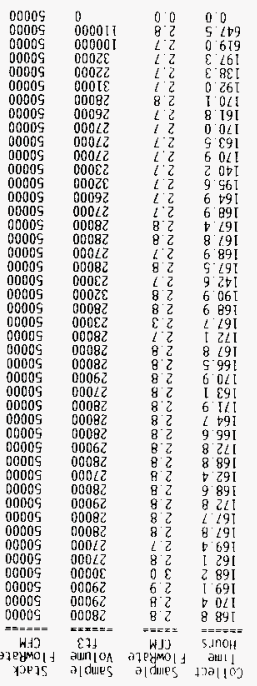

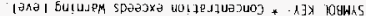

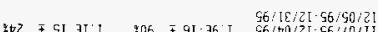

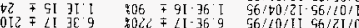

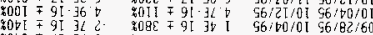

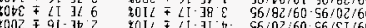

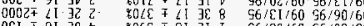

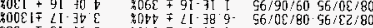

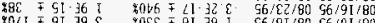

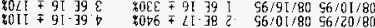

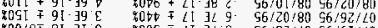

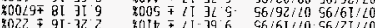

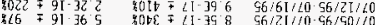

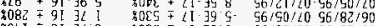

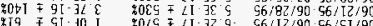

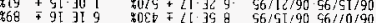

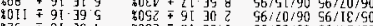

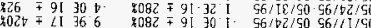

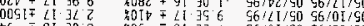

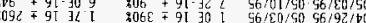

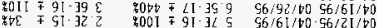

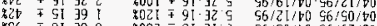

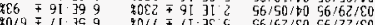

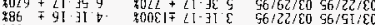

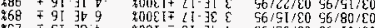

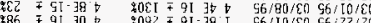

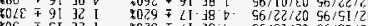

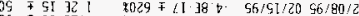

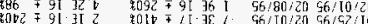

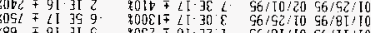

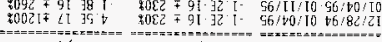

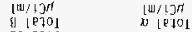
b. 3010 รä1e0 $150 / 410$ SLand bH ti.JPM [2: II $96 ; 12: 90$ 


\section{WHC-SD-EN-TI-308 Rev.0}

WHC ENVIRONMENTAL RELEASE SUMMARY

696-W-2: WSCF, NSI COUNTING ROOM EXHAUSTER (WOII)

$01 / 01 / 95$ through $12 / 31 / 95$

Total System Flow: $2.2 \mathrm{E}+10$ Liters $=7.9 \mathrm{E}+08$ Cubic $\mathrm{Ft}$

The following factor(s) has (or have) been applied to the radionuclide concentrations used in the calculation of this release to account for sampling filter efficiency, line and bend loss, and humidity. The concentrations vary inversely with the size of the factor (s).

$$
\text { Particulates: } \quad 0.73
$$

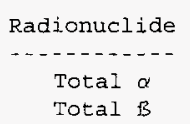
Release, Ci -......... 6. 1E-09
3. $6 E-08$

Release, $C \dot{I}$
$6.1 E-09$
$3.6 \mathrm{E}-08$




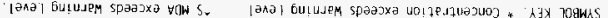

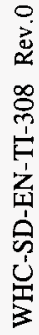

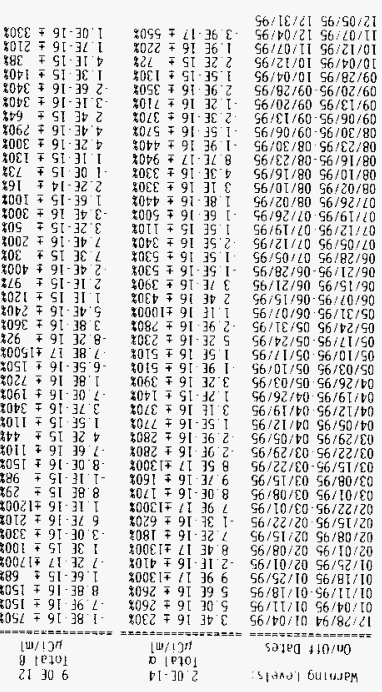

[द $1196 ;(t), y 0$ 
WHC-SD-EN-TI-308 Rev.0

This page intentionally left blank. 
WHC-SD-EN-TI-308 Rev.0

2.5.6 PFP 


\section{WHC-SD-EN-TI-308 Rev.0}

ENVIRONMENTAL RELEASE SUMMARY REPORT

291-Z-1: 232-Z, 234-5, 236-Z, 242-Z PROCESS \& VENT (X810, z810)

$01 / 01 / 95$ through $12 / 31 / 95 \star \star \star$

Total system Flow: $3 \cdot 7 \mathrm{E}+12$ Liters $=1 \cdot 3 \mathrm{E}+11$ Cubic $\mathrm{Ft}$

The following factor(s) has (or have) been applied to the radionuclide concentrations used in the calculation of this release to account for sampling filter efficiency, line and bend loss, and humidity. The concentrations vary inversely with the size of the factor (s).

$$
\text { Particulates: } \quad 0.73
$$

$\begin{array}{cccc}\text { Radionuclice } & \text { Release, Ci } & \begin{array}{c}\text { Concentrations, } \mu \mathrm{Ci} / \mathrm{ml} \\ \text { Average }\end{array} & \text { Peak } \\ { }^{238} \mathrm{Pu} & 2.3 \mathrm{E}-06 & 4.5 \mathrm{E}-16 & 7.9 \mathrm{E}-16 \\ 239 / 240 \mathrm{Pu} & 7.6 \mathrm{E}-05 & 1.5 \mathrm{E}-14 & 2.1 \mathrm{E}-14 \\ 241 \mathrm{Pu} & 2.0 \mathrm{E}-04 & 3.9 \mathrm{E}-14 & 6.9 \mathrm{E}-14 \\ { }^{241} \mathrm{Am} & 1.5 \mathrm{E}-05 & 2.9 \mathrm{E}-15 & 4.0 \mathrm{E}-15 \\ \text { Total } \alpha & 1.2 \mathrm{E}-04 & 2.4 \mathrm{E}-14 & 1.5 \mathrm{E}-13 \\ \text { Total } \mathrm{B} & 9.5 \mathrm{E}-06 & 1.9 \mathrm{E}-15 & 1.1 \mathrm{E}-14 \\ & & & \\ & & & \end{array}$


36: $21 / 9611: 22$

$29171.2337 .2345 .736 \%$. 242 Z PROCESS \& VENT (7B10)

\begin{tabular}{|c|c|c|c|c|c|c|}
\hline $\begin{array}{l}\text { Warming l puels } \\
\text { Oncori vates }\end{array}$ & 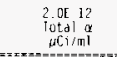 & 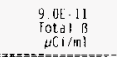 & $\begin{array}{c}\text { Collect } \\
1 \text {, rite' } \\
\text { Hours }\end{array}$ & $\begin{array}{c}\text { Sample } \\
\text { F Iowkat.e } \\
\text { CFM }\end{array}$ & $\begin{array}{l}\text { Sample } \\
\text { volunee } \\
\text { fi3 }\end{array}$ & $\begin{array}{l}\text { Stack } \\
\text { Flowkat } \\
\text { CFM }\end{array}$ \\
\hline 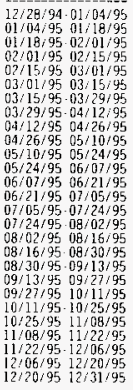 & 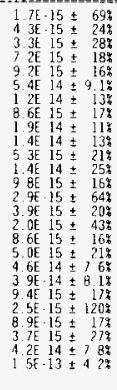 & $\begin{array}{l}4.2 \mathrm{E} \cdot 16 \pm 220 \% \\
4.0 \mathrm{E}-16 \pm 100 \% \\
5.5 \mathrm{E} \cdot 16 \pm 90 \% \\
9.1 \mathrm{E} .16 \pm 50 \% \\
3.1 \mathrm{~F} \cdot 16 \pm 130 \% \\
43 \mathrm{BE} \cdot 15 \pm 31 \% \\
1.2 \mathrm{E}-15 \pm 39 \% \\
2.15 .15 \pm 27 \% \\
1.7 \mathrm{E} .15 \pm 35 \% \\
2.4 \mathrm{E}-16 \pm 170 \% \\
4.8 \mathrm{E} 16 \pm 100 \% \\
4.7 \mathrm{E}-16 \pm 97 \%\end{array}$ & $\begin{array}{l}173.1 \\
331.1 \\
33 \% .7 \\
335.1 \\
336.0 \\
334.4\end{array}$ & $\begin{array}{l}1.8 \\
1.8 \\
1.7 \\
1.8 \\
1.7 \\
0.9\end{array}$ & $\begin{array}{r}51000 \\
23000 \\
33000 \\
380000 \\
32000 \\
33000 \\
33000 \\
33000 \\
33000 \\
32000 \\
33000 \\
37000 \\
3700\end{array}$ & \\
\hline
\end{tabular}

¿5 MDA exceevs warning Level.
41:01:95 10 12:31:95

Page

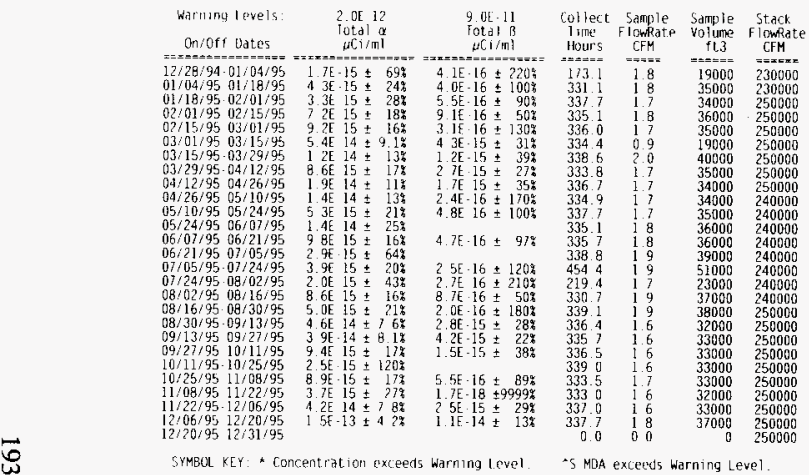

? Reported MiA is negative

^ Stack Flowfate of previous Samle 
WHC-SD-EN-TI-308 Rev.0
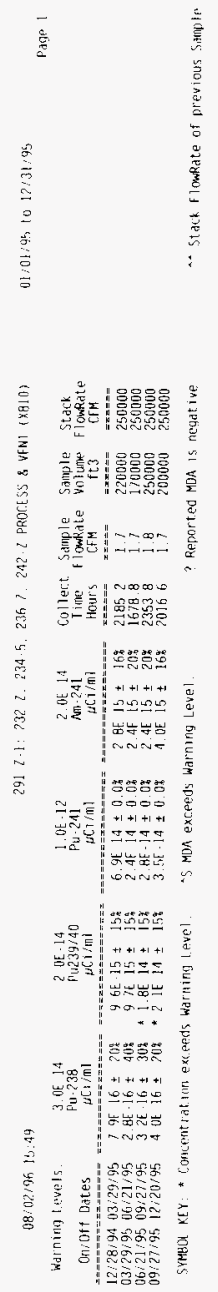
WHC-SD-EN-TI-308 Rev.0

$08 / 02 / 96$

ENVIRONMENTAL RELEASE SUMMARY REPORT

296-Z-3: 241-Z SUMP \& VESSEL VENTS (X813, Z813)

$01 / 01 / 95$ through $12 / 31 / 95$

Total system Flow: $1.4 \mathrm{E}+10$ Liters $=4.9 \mathrm{E}+08$ Cubic Ft

The following factor(s) has (or have) been applied to the radionuclide concentrations used in the calculation of this release to account for sampling filter efficiency, line and bend loss, and humidity. The concentrations vary inversely with the size of the factor $(s)$.

Particulates: $\quad 0.73$

\begin{tabular}{|c|c|c|c|}
\hline & & \multicolumn{2}{|c|}{ Concentrations, $\mu \mathrm{Ci} / \mathrm{ml}$} \\
\hline Radionuclide & Release, Ci & Average & Peak \\
\hline---------- & --------- & $----\cdots$ & ----- \\
\hline${ }^{238} \mathrm{Pu}$ & 3. $5 E-08$ & 1. 8E-15 & $4.1 E-15$ \\
\hline $239 / 240 \mathrm{Pu}$ & 3. $1 E-08$ & $1.6 \mathrm{E}-15$ & 3. $3 E-15$ \\
\hline${ }^{24}{ }^{\mathrm{Pu}}$ & 3. $1 \mathrm{E}-06$ & $1.6 \mathrm{E}-13$ & $3.6 E-13$ \\
\hline${ }^{241} \mathrm{Am}$ & 1. $5 E-08$ & $7.9 E-16$ & $1.8 E-15$ \\
\hline Total $\alpha$ & $8.1 \mathrm{E}-08$ & $4.3 E-15$ & $1.8 \mathrm{E}-14$ \\
\hline Total B & 4. $0 E-08$ & $2.1 E-15$ & $1.2 E-1.4$ \\
\hline
\end{tabular}

*** Values projected for period $12 / 31 / 95$ to $12 / 31 / 95$. 
पh/? $1 / 46$ 1!:2?

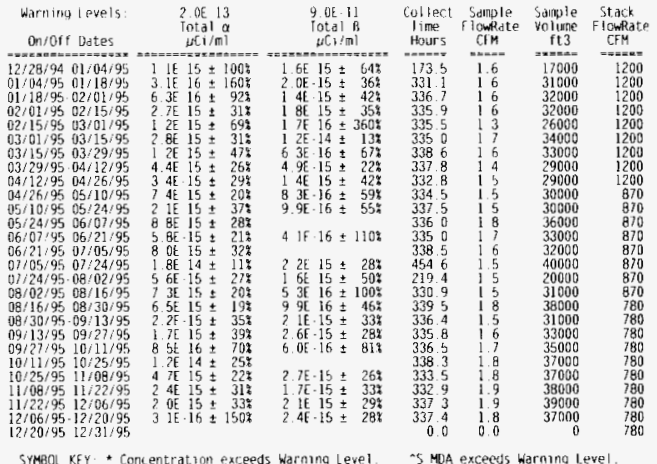

01/01/95 to 12/31/94

Pag̣e :
? Reported MisA is regat.1ye. 
WHC-SD-EN-TI-308 Rev.0
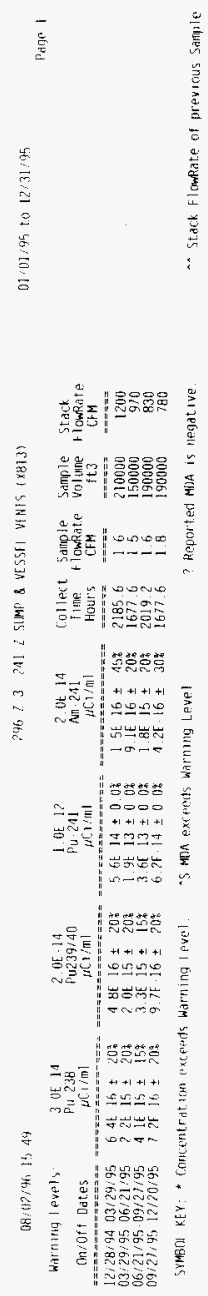
WHC-SD-EN-TI-308 Rev.0

WHC ENVIRONMENTAL RELEASE SUMMARY

296-Z-5: 2736-ZB BUILDING EXHAUST (Z913, X913)

$01 / 01 / 95$ through $12 / 31 / 95$

Total System Flow: 1.4E+11 Liters $=4.9 E+09$ Cubic Ft

The following factor(s) has (or have) been applied to the radionuclide concentrations used in the calculation of this release to account for sampling Eilter efficiency, line and bend loss, and humidity. The concentrations vary inversely with the size of the factoris).

Particulates:

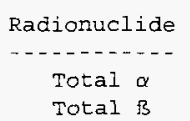

\author{
Release, Ci \\ -.-----... \\ 1. $9 E-08$ \\ 5. $6 \mathrm{E}-08$
}

0.73

$\begin{array}{cc}\text { Concentrations, } \mu \mathrm{Ci} / \mathrm{mI} \\ \text { Average } & \text { Peak } \\ - & ------ \\ 9.9 E-17 & 9.1 \mathrm{E}-16 \\ \text { 3. OE-16 } & 2.6 \mathrm{E}-15\end{array}$


06:21196 11:27

\begin{tabular}{|c|c|c|c|c|c|c|}
\hline $\begin{array}{l}\text { Warming levels. } \\
\text { Oniofl Dates }\end{array}$ & 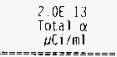 & 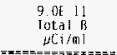 & $\begin{array}{l}\text { Collect } \\
\text { Tine } \\
\text { Hours }\end{array}$ & $\begin{array}{l}\text { jample } \\
\text { Flowrate } \\
\text { CFHA }\end{array}$ & $\begin{array}{l}\text { Satnple } \\
\text { volumae } \\
\mathrm{it3}\end{array}$ & $\begin{array}{l}\text { Stack } \\
\text { rlowklate } \\
\text { CFM } \\
\text { CF= }\end{array}$ \\
\hline 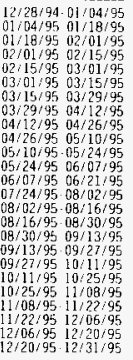 & 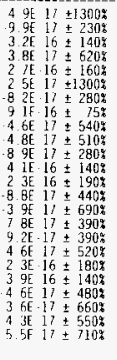 & 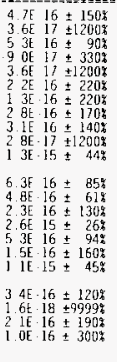 & $\begin{array}{l}171.4 \\
331.3 \\
341.7 \\
331.1 \\
336.8 \\
334.6 \\
337.7 \\
338.5 \\
331.3 \\
336.2 \\
337.5 \\
334.0 \\
337.4 \\
220.0 \\
330.1 \\
338.1 \\
336.1 \\
335.8 \\
336.2 \\
337.9 \\
336.2 \\
332.8 \\
337.2 \\
337.3 \\
0.0\end{array}$ & $\begin{array}{r}17 \\
16 \\
1.7 \\
1.7 \\
1.7 \\
1.7 \\
0.0\end{array}$ & $\begin{array}{l}18=000 \\
360000 \\
360000 \\
35000 \\
35000 \\
350000 \\
35000 \\
30000 \\
32000 \\
33000 \\
32000 \\
32000 \\
32000 \\
20000 \\
300000 \\
38000 \\
33000 \\
33000 \\
33000 \\
34000 \\
35000 \\
34000 \\
34000 \\
34000 \\
3400\end{array}$ & $\begin{array}{l}13000 \\
13000 \\
10000 \\
10000 \\
10000 \\
100000 \\
10000 \\
10000 \\
10000 \\
12000 \\
12000 \\
12000 \\
12000 \\
12000 \\
12000 \\
5500 \\
5500 \\
5500 \\
5500 \\
5500 \\
5500 \\
5500 \\
5500 \\
5500 \\
5500\end{array}$ \\
\hline
\end{tabular}

¿ 5 MOA exceeds Warning Level.
$01: 01: 951012 / 31 \% 94$

Pagp

? Reported MIOA is negative.

A Stack flowrate of previous Sample 
WHC-SD-EN-TI-308 Rev.0

WHC ENVIRONMENTAL RELEASE SUMMARY

$06 / 14 / 96$

$13: 18$

296-Z-6: 2736-ZA BUILDING EXHAUST (z802)

$01 / 01 / 95$ through $12 / 31 / 95$

Total system Flow: $1.3 E+21$ Liters $=4.7 E+09$ Cubic Ft

The following factor(s) has (or have) been applied to the radionuclide concentrations used in the calculation of this release to account for sampling filter efficiency, 1 ine and bend loss, and humidity. The concentrations vary inversely with the size of the factor (s).

$$
\text { Particulates: } \quad 0.73
$$

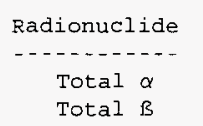
Release, Ci
-.-.-.....-
4. 2E-08
1. $6 \mathrm{E}-07$

$\begin{array}{cccc}\text { Particulates: } & 0.73 \\ \text { Radionuclide } & \text { Release, Ci } & \begin{array}{c}\text { Concentrations, } \mu \mathrm{Ci} / \mathrm{m} 1 \\ \text { Average }\end{array} \text { Peak } \\ \text { Total } \alpha & 4.1 \mathrm{E}-08 & 2.2 \mathrm{E}-16 & 1.7 \mathrm{E}-15 \\ \text { Total } B & 1.6 \mathrm{E}-07 & 8.5 \mathrm{E}-16 & 4.7 \mathrm{E}-15\end{array}$


WHC-SD-EN-TI-308 Rev.0

WHC ENVIRONMENTAL RELEASE SUMMARY

296-Z-14: 232-Z BUILDING EXHAUST (Z814)

$01 / 01 / 95$ through $12 / 31 / 95$

Total system Flow: $3.0 \mathrm{E}+10$ Liters $=1.1 \mathrm{E}+09$ Cubic Ft

The following factor(s) has (or have) been applied to the radiomuclide concentrations used in the calculation of this release to account for sampling filter efficiency, line and bend loss, and humidity. The concentrations vary inversely with the size of the factor $(s)$.

Particulates: $\quad 0.73$

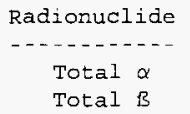

Total $\alpha$

Total $B$
Release, Ci -.........
8. $6 \mathrm{E}-09$
4. $9 \mathrm{E}-08$

\begin{tabular}{cc} 
Concentrations, $\mu \mathrm{Ci} / \mathrm{ml}$ \\
Average & Peak \\
\hdashline$-1 .--$ & ----- \\
$2.1 \mathrm{E}-16$ & $1.0 \mathrm{E}-15$ \\
$1.2 \mathrm{E}-15$ & 4. $1 \mathrm{E}-15$
\end{tabular}


$06: 31196 \quad 11: 22$

\begin{tabular}{|c|c|c|c|c|c|c|}
\hline $\begin{array}{l}\text { Warning l ewels: } \\
\text { on } 0 \text { off Dates }\end{array}$ & 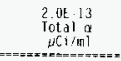 & 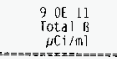 & $\begin{array}{l}\text { Cullect } \\
\text { Iime } \\
\text { Hours } \\
\text { tours }\end{array}$ & $\begin{array}{l}\text { Sariple } \\
\text { flowplate } \\
\text { CrM } \\
===-\end{array}$ & $\begin{array}{l}\text { Sumple } \\
\text { volume } \\
\mathrm{ft} 3 \\
x===\end{array}$ & $\begin{array}{l}\text { Stad } \\
\text { F lowfate } \\
\text { CFM } \\
\text { - }== \pm= \pm\end{array}$ \\
\hline 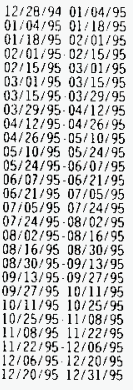 & 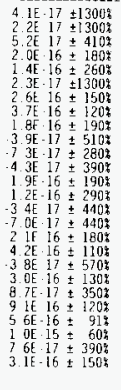 & 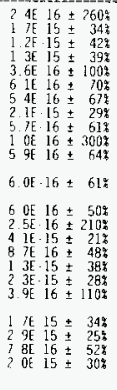 & $\begin{array}{l}172.7 \\
331.0 \\
340.7 \\
331.3 . \\
337.3 \\
334.6 \\
337.4 \\
335.8 \\
334.4 \\
335.4 \\
336.1 \\
336.0 \\
336.6 \\
338.5 \\
45.2 \\
219.6 \\
330.7 \\
338.6 \\
336.5 \\
335.6 \\
336.4 \\
338.3 \\
334.8 \\
333.0 \\
337.3 \\
337.4 \\
0.0\end{array}$ & $\begin{array}{r}==- \\
2.1 \\
2.0 \\
1.9 \\
1.9 \\
1.9 \\
1.9 \\
1.9 \\
2.0 \\
2.0 \\
2.0 \\
1.9 \\
1.9 \\
1.9\end{array}$ & $\begin{array}{l}2== \\
21000 \\
41000 \\
39000 \\
37000 \\
39000 \\
39000 \\
39000 \\
40000 \\
40000 \\
40000 \\
39000 \\
38000 \\
39000 \\
410000 \\
52000 \\
25000 \\
37000 \\
3900 \\
39000 \\
38000 \\
38000 \\
40000 \\
35000 \\
37000 \\
38000 \\
38000 \\
0\end{array}$ & $\begin{array}{l}-x==0 \\
16000 \\
1600 \\
1800 \\
1800 \\
1800 \\
1800 \\
1800 \\
1800 \\
1800 \\
2100 \\
2100 \\
2100 \\
2100 \\
2100 \\
2100 \\
2100 \\
2100 \\
2100 \\
2100 \\
2100 \\
2100 \\
2100 \\
2100 \\
2100 \\
2100 \\
2100 \\
2100\end{array}$ \\
\hline
\end{tabular}

$01: 01 / 95$ to $17 \% 31: 95$

Page 


\section{WHC-SD-EN-TI-308 Rev.0}

WHC ENVIRONMENTAL RELEASE SUMMARY

296-Z-15: 243-Z LOW LEVEL WASTE WATER TREATMENT FACI (Z915)

$$
01 / 01 / 95 \text { through } 12 / 31 / 95
$$

Total system Flow: $2.4 \mathrm{E}+10$ Liters $=8.4 \mathrm{E}+08$ Cubic Ft

The following factor(s) has (or have) been applied to the radionuclide concentrations used in the calculation of this release to account for sampling filter efficiency, line and bend loss, and humidity. The concentrations vary inversely with the size of the factor (s).

$\begin{array}{cccc}\text { Particulates: } & 0.73 \\ \text { Radionuclide } & \text { Release, Ci } & \begin{array}{c}\text { Concentrations, } \mu \mathrm{Ci} / \mathrm{ml} \\ \text { Average }\end{array} \\ \text { Peak } \\ \text { Total } \alpha & 3.9 \mathrm{E}-09 & 1.2 \mathrm{E}-16 & 1.2 \mathrm{~B}-16\end{array}$




\section{WHC-SD-EN-TI-308 Rev.0}
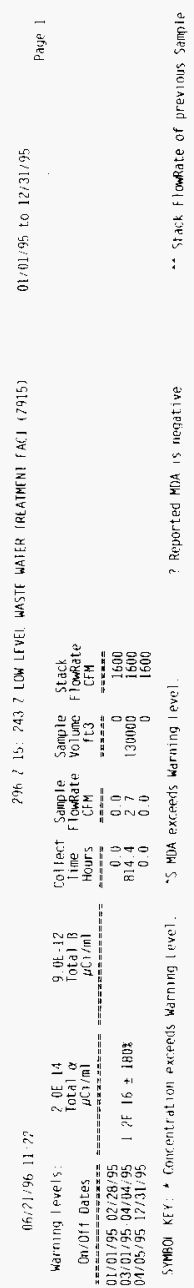
WHC-SD-EN-TI-308 Rev.0

This page intentionally left blank. 
WHC-SD-EN-TI-308 Rev.0

2.5.7 200-W BHI Facilities 


\section{WHC-SD-EN-TI-308 Rev.0}

WHC ENVIRONMENTAL RELEASE SUMMARY

291-S-1: 202-S CANYON EXHAUST (S006)

$01 / 01 / 95$ through $12 / 31 / 95$

Total System Flow: $3.1 \mathrm{E}+11$ Liters $=1.1 \mathrm{E}+10$ Cubic Ft

The following factor(s) has (or have) been applied to the radionuclide concentrations used in the calculation of this release to account, for sampling filter efficiency, line and bend loss, and humidity. The concentrations vary inversely with the size of the factor(s).

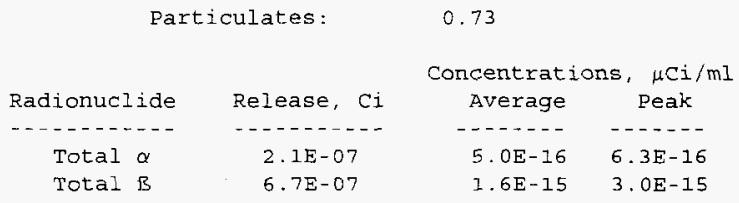


$06 / 21 / 9613.21$

\begin{tabular}{|c|c|c|c|c|c|c|}
\hline $\begin{array}{l}\text { Warning l evels: } \\
\text { Onroff Dates } \\
\text { Ontor }\end{array}$ & 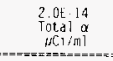 & $\begin{array}{l}9.0 \mathrm{e} 12 \\
\text { ottal is } \\
\text { of } \mathrm{i} / \mathrm{ml}\end{array}$ & $\begin{array}{l}\text { cot lect } \\
\text { Tlume } \\
\text { Hours } \\
x===-\end{array}$ & 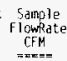 & $\begin{array}{c}\text { Salpple } \\
\text { Volume } \\
\text { ft.3 } \\
=x+==\end{array}$ & $\begin{array}{l}\text { Stack } \\
\text { Flowflat } \\
\text { CFM } \\
=x=0\end{array}$ \\
\hline $\begin{array}{l}03 / 14 / 95 \\
03 / 92 / 95 \\
05 / 0019\end{array}$ & $63 E 16 \pm$ & $42 F \cdot 16 \pm 17 D q$ & $\begin{array}{r}0=-3 \\
00 \\
168.2 \\
0.0\end{array}$ & & 8000 & \\
\hline 05 & 4. $2 E 16 \pm 190 \%$ & 1 GE $15 \pm 56 \%$ & & & 1600 & \\
\hline $\begin{array}{l}07 / 04 / 95 \\
-07 / 12 / 95\end{array}$ & 1.BE-16 & $36 E 16 \pm 2309$ & $\begin{array}{l}0 \\
170\end{array}$ & $\begin{array}{l}0.6 \\
1.7\end{array}$ & $\begin{array}{r}0 \\
17000\end{array}$ & \\
\hline $\begin{array}{l}07 / 13 / 95 \\
10 / 02 / 95 \\
10 / 0\end{array}$ & 6. $2716 \pm 140 \%$ & $3.0515 \pm 37 \%$ & $\begin{array}{r}0.0 \\
168.0\end{array}$ & 0. & 000 & \\
\hline & & & & & & \\
\hline
\end{tabular}

5YMBO KEY: * Concentration exceeds warning Level. "S MDA excceds Warning Level.
$01 / 01 / 95$, to $12: 31 / 95$

Psge 1

Repurted MDA is negative 


\section{WHC-SD-EN-TI-308 Rev.0}

WHC ENVIRONMENTAL RELEASE SUMMARY

296-S-2: 202-S NORTH SAMPL. GAL., HOOD, \& PR ROOM (S032)

$01 / 01 / 95$ through $12 / 31 / 95$

Total System Flow: $1.9 E+10$ Liters $=6.8 E+08$ Cubic Ft

The following factor (s) has (or have) been applied to the radionuclide concentrations used in the calculation of this release to account for sampling filter efficiency, line and bend loss, and humidity. The concentrations vary inversely with the size of the factor $(s)$.

Particulates:

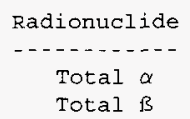

Release, Ci
6. $9 E-09$
$1.6 E-08$

Release, $\mathrm{CI}$

1. $6 E-08$
0.73

\begin{tabular}{cc} 
Concentrations, $\mu \mathrm{Ci} / \mathrm{ml}$ \\
Average & Peak \\
\hline$-16 \mathrm{E}-16$ & $6.2 \mathrm{E}-16$ \\
$2.0 \mathrm{E}-16$ & $8.9 \mathrm{E}-16$
\end{tabular}


BG:?1/46 11 ? 11

\begin{tabular}{|c|c|c|c|c|c|c|}
\hline 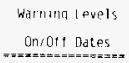 & 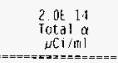 & 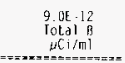 & $\begin{array}{l}\text { loplect } \\
\text { I ime } \\
\text { Hours } \\
==-=-=x\end{array}$ & $\begin{array}{l}\text { Simple } \\
\text { f lowrate } \\
\text { CFM }\end{array}$ & $\begin{array}{l}\text { Sumple } \\
\text { volume } \\
\text { vit3 } \\
==-\end{array}$ & $\begin{array}{l}\text { Stack } \\
\text { F lowpate } \\
\text { CFM }\end{array}$ \\
\hline 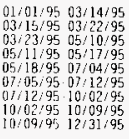 & $\begin{array}{l}1.7 \mathrm{E} 16 \pm 400 \% \\
1.9 \mathrm{E} \cdot 16 \pm 400 \% \\
17116 \pm 380 \% \\
3.2 \mathrm{E} 16 \pm 37 \\
6.2 \mathrm{~F} 16 \pm 140 \%\end{array}$ & $\begin{array}{l}6.4 E 16 \pm 130 \% \\
2.7 F 16 \pm 320 \% \\
84[-16 \pm 100 \% \\
4.8165 \pm 278 \\
8.4616 \pm 110 \%\end{array}$ & $\begin{array}{r}0.0 \\
168.1 \\
00 \\
143.0 \\
100 \\
190.0 \\
1966 \\
168.5 \\
0.0\end{array}$ & $\begin{array}{ll}0 & 0 \\
1 & 7 \\
0.0 \\
1 & 7 \\
0 & 0 \\
1 & 7 \\
1 & 7 \\
1 & 8 \\
0 & 0\end{array}$ & $\begin{array}{r}17000 \\
14000 \\
1400 \\
18000 \\
200000 \\
18000 \\
0\end{array}$ & $\begin{array}{l}770 \\
770 \\
770\end{array}$ \\
\hline
\end{tabular}

sYMBOL KFY: * Concentration exceeds Warning Level. 


\section{WHC-SD-EN-TI-308 Rev.0}

\section{WHC ENVIRONMENTAL RELEASE SUMMARY}

296-S-4: 202-S: SWF LOBBY, DECON RM, REG. SHOP (S008)

$$
01 / 01 / 95 \text { through } 12 / 31 / 95
$$

Total System Flow: $4.4 \mathrm{E}+09$ Liters $=1.6 \mathrm{E}+08$ Cubic Ft

The following factor(s) has (or have) been applied to the radionuclide concentrations used in the calculation of this release to account for sampling filter efficiency, line and bend loss, and humidity. The concentrations vary inversely with the size of the factor (s).

Particulates:

Radionuclide Total $\alpha$ Total B

Release, Ci
4. $4 \mathrm{E}-09$
$1.4 \mathrm{E}-08$

0.73

\begin{tabular}{|c|c|}
\hline $\begin{array}{l}\text { antrat } \\
\text { Average }\end{array}$ & is, $\begin{array}{c}\mu \mathrm{Ci} / \\
\text { Peak }\end{array}$ \\
\hline$-\cdots$ & $--\ldots--$ \\
\hline 7. $3 E-16$ & 7. $3 \mathrm{E}-16$ \\
\hline $2.3 E-15$ & $2.3 E-15$ \\
\hline
\end{tabular}


WHC-SD-EN-TI-308 Rev.0
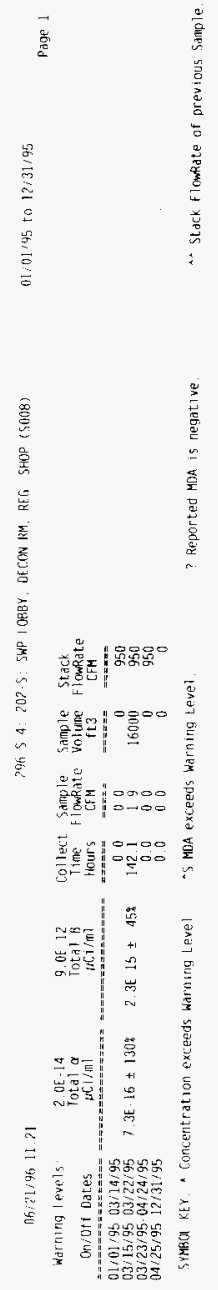


\section{WHC-SD-EN-TI-308 Rev.0}

WHC ENVIRONMENTAL RELEASE SUMMARY

296-S-6: UNEILTERED AIR FROM 202-S SILO (S004)

$01 / 01 / 95$ through $12 / 31 / 95$

Total system Flow: 1.2E +10 Liters $=4 \cdot 3 \mathrm{E}+08$ Cubic Ft

The following factor(s) has (or have) been applied to the radionuclide concentrations used in the calculation of this release to account for sampling filter efficiency, line and bend loss, and humidity. The concentrations vary inversely with the size of the factor $(s)$.

$$
\text { Particulates: } \quad 0.73
$$

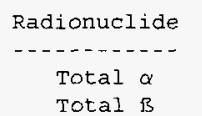

Total is

Release, Ci
$2.2 E-08$
8.0E-08

\begin{tabular}{cc}
$\begin{array}{c}\text { Concentrations, } \mu \mathrm{Ci} / \mathrm{ml} \\
\text { Average }\end{array}$ & Peak \\
\hline $1.3 E-15$ & $2.2 \mathrm{E}-15$ \\
4.8E-15 & $7.1 \mathrm{E}-15$
\end{tabular}


WHC-SD-EN-TI-308 Rev.0
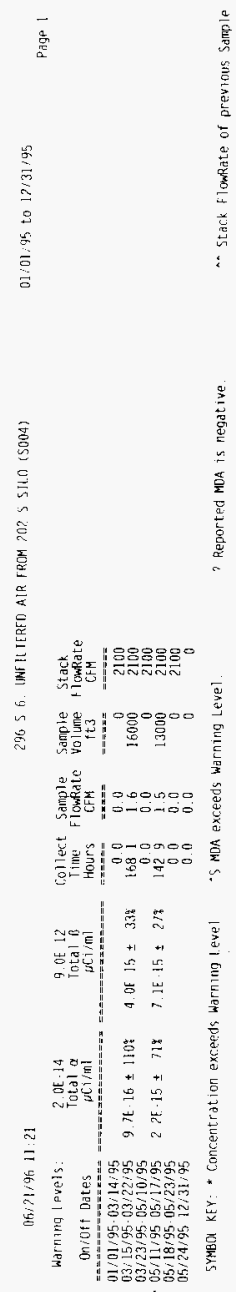
WHC-SD-EN-TI-308 Rev.0

WHC ENVIRONMENTAL RELEASE SUMMARY

296-S-7W \& 296-S-7E: 233-S BUILDING EXHAUSTERS (S016, SO15)

$$
01 / 01 / 95 \text { through } 12 / 31 / 95
$$

Total System Flow: $1.4 E+11$ Liters $=4.8 E+09$ Cubic Ft

$\begin{array}{ccc}\text { Radionuclide } & \text { Halflife } & \text { Release, Ci } \\ \text { Total } \alpha & \text { n/a } & 5.6 E-07 \\ \text { Total } \beta & \text { n/a } & 2.9 E-07\end{array}$


06:\%1:96 11.71

\begin{tabular}{|c|c|c|c|c|c|c|}
\hline $\begin{array}{l}\text { wornulg levels. } \\
\text { Onyorf Oates }\end{array}$ & $\begin{array}{l}2 \text { oE } 13 \\
\text { Iotal a } \\
\mu C i / m ?\end{array}$ & $\begin{array}{l}9.0 E \text { ll } \\
\text { Total } \\
\mu C, i / \text { all }\end{array}$ & $\begin{array}{l}\text { Collect. } \\
\text { Times } \\
\text { thours }\end{array}$ & $\begin{array}{l}\text { Sample } \\
\text { Flowkate } \\
\text { CFM }\end{array}$ & $\begin{array}{l}\text { Sample } \\
\text { volune } \\
\text { ft3 }\end{array}$ & $\begin{array}{l}\text { Stuck } \\
\text { riowkate } \\
\text { CFM }\end{array}$ \\
\hline 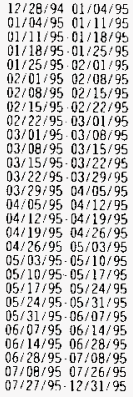 & 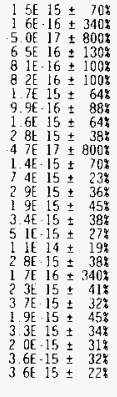 & 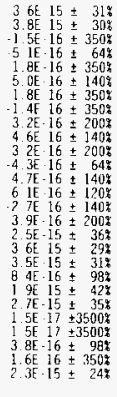 & 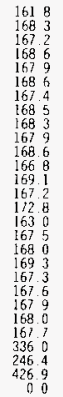 & $\begin{array}{l}1.6 \\
2.0 \\
6.0\end{array}$ & $\begin{array}{l}20000 \\
2000 \\
20000 \\
19000 \\
20000 \\
20000 \\
20000 \\
21000 \\
22000 \\
22000 \\
22000 \\
22000 \\
22000 \\
22090 \\
23000 \\
18000 \\
22000 \\
23000 \\
22000 \\
20000 \\
22000 \\
22000 \\
22000 \\
22000 \\
44000 \\
23000 \\
50000 \\
200\end{array}$ & $\begin{array}{l}9500 \\
9500 \\
9500 \\
9500 \\
9500 \\
9500 \\
9500 \\
9500 \\
9500 \\
9500 \\
9500 \\
95500 \\
9500 \\
9500 \\
9500 \\
9500 \\
9500 \\
9500 \\
9500 \\
9500 \\
9500 \\
9500 \\
9000 \\
9000 \\
9000 \\
9000 \\
9900 \\
9000\end{array}$ \\
\hline
\end{tabular}

017/41:95 tor $12 / 31 / 95$

H'age I

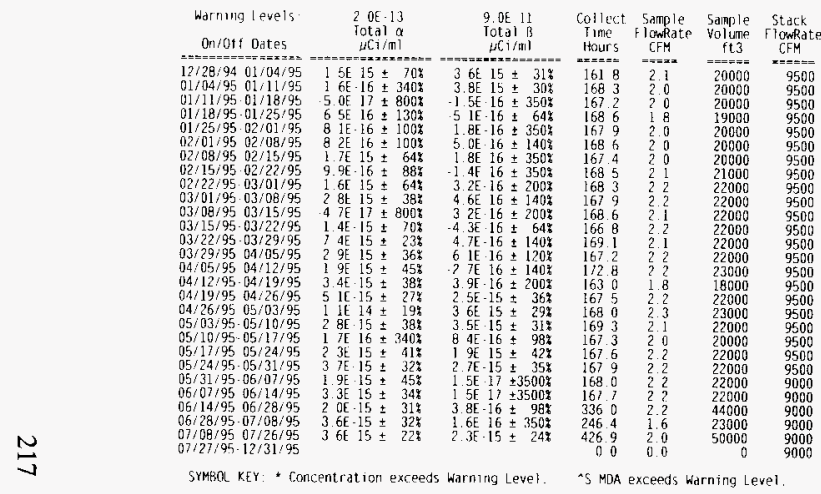




\section{WHC-SD-EN-TI-308 Rev.0}

WHC ENVIRONMENTAL RELEASE SUMMARY

291-U-1: 221-U CANYON \& CELLS (U771, X771)

$01 / 01 / 95$ through $12 / 31 / 95$

Total System Flow: $3.7 E+11$ Liters $=1.3 E+10$ Cubic Ft

The following factor(s) has (or have) been applied to the radionuclide concentrations used in the calculation of this release to account for sampling filter efficiency, line and bend loss, and humidity. The concentrations vary inversely with the size of the factor (s).

$$
\text { Particulates: } \quad 0.73
$$

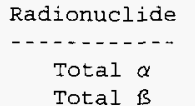
Release, $\mathrm{Ci}$ - - . . . - . . .
2. $5 \mathrm{E}-07$
5. $4 E-05$

\begin{tabular}{cc} 
Concentrations, $\mu \mathrm{Ci} / \mathrm{ml}$ \\
Average & Peak \\
\hdashline---- & ----- \\
$5.0 \mathrm{E}-16$ & $6.3 \mathrm{E}-16$ \\
$1.1 \mathrm{E}-13$ & $1.7 \mathrm{E}-13$
\end{tabular}


06/21/96 11:?

\begin{tabular}{|c|c|c|c|c|c|c|}
\hline $\begin{array}{l}\text { Walming l payels } \\
\text { On/oft Dates }\end{array}$ & 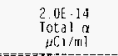 & 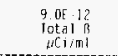 & $\begin{array}{l}\text { rollect } \\
\text { I ine } \\
\text { Hours }\end{array}$ & $\begin{array}{c}\text { Sample } \\
\text { Flomidate } \\
\text { CFM }\end{array}$ & $\begin{array}{l}\text { Sample } \\
\text { Waluine } \\
\mathrm{ft} 3\end{array}$ & $\begin{array}{l}\text { St.ack } \\
\text { FlawRate } \\
\text { CFM }\end{array}$ \\
\hline 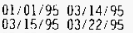 & $6.1 E 16 \pm 130 \%$ & $7.9 E-14 \pm 6.4$ & 1690 & 00 & 800 & $\begin{array}{l}23000 \\
23000\end{array}$ \\
\hline $23 / 95.05 / 09 / 95$ & $40=16$ & 715 & & & & \\
\hline 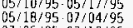 & 4.01 .16 & $7: 16 \cdot 14 \pm 76$ & $\begin{array}{r}15.8 .6 \\
0.0\end{array}$ & 0.0 & $1 / 000$ & $\begin{array}{l}23000 \\
23000\end{array}$ \\
\hline $\begin{array}{l}07 / 05 / 95 \cdot 07 / 12 / 95 \\
07 / 13 / 95 \\
0\end{array}$ & $1.5 \mathrm{E}$ & $4.2 F-14 \pm 857$ & 15.94 & 2. 0 & 21000 & 23000 \\
\hline $\begin{array}{l}10 / 02 / 95 \quad 1006 / 95 \\
10 / 10 / 95 \\
12 / 31 / 95\end{array}$ & 6. $3 E 16$ & 1. IE $13 \pm 448$ & $\begin{array}{r}1665 \\
165 \\
00\end{array}$ & $\begin{array}{l}10 \\
10.0 \\
0.0\end{array}$ & 19000 & $\begin{array}{l}27000 \\
27000\end{array}$ \\
\hline
\end{tabular}

5YMEQ KEY * Concentration exceeds Warning l evel
291 U. 1: ?21 II LANYON \& CEHIS IUHI

¿5 MaA exceeds warning Level.
? Reported MOA is negative
01/01: 45 to 13/31/95.

Page 1

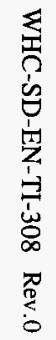


WHC-SD-EN-TI-308 Rev.0

This page intentionally left blank. 
WHC-SD-EN-TI-308 Rev.0

\subsection{Area}


WHC ENVIRONMENTAL RELEASE SUMMARY

340-NT-EX: 340 Vault Tank Vent Exhaust (F002, F017, F602)

$01 / 01 / 95$ through $12 / 31 / 95$

Total system Flow: $2.9 \mathrm{E}+10$ Liters $=1.0 \mathrm{E}+09$ cubic Ft

The following factor(a) has (or have) been applied to the radionuclide concentrations used in the calculation of this release to account for sampling filter efficiency, line and bend loss, and humidity. The concentrations vary inversely with the size of the factor(s).

$$
\begin{array}{ll}
\text { Particulates: } & 0.73 \\
\text { Halogens: } & 0.87
\end{array}
$$

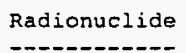

${ }^{90} \mathrm{Sr}$

$131_{\mathrm{I}}$

${ }^{137} \mathrm{Cs}$

$239 / 240 \mathrm{Pu}$

Total a

Total $\beta$

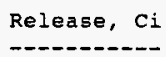

ND

ND

ND

ND

6. $7 \mathrm{E}-0 \mathrm{~B}$

1. $3 E-07$
Concentrations, $\mu \mathrm{Ci} / \mathrm{ml}$

Average peak

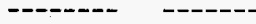

<3. 3E-16 n/a

$<4.2 \mathrm{E}-14 \quad \mathrm{n} / \mathrm{a}$

<4.2E-16 $\mathrm{n} / \mathrm{a}$

$<3.9 \mathrm{E}-17 \quad \mathrm{n} / \mathrm{a}$

1. $7 E-153.4 E-14$

3. $2 \mathrm{E}-15 \quad 5.8 \mathrm{E}-14$ 


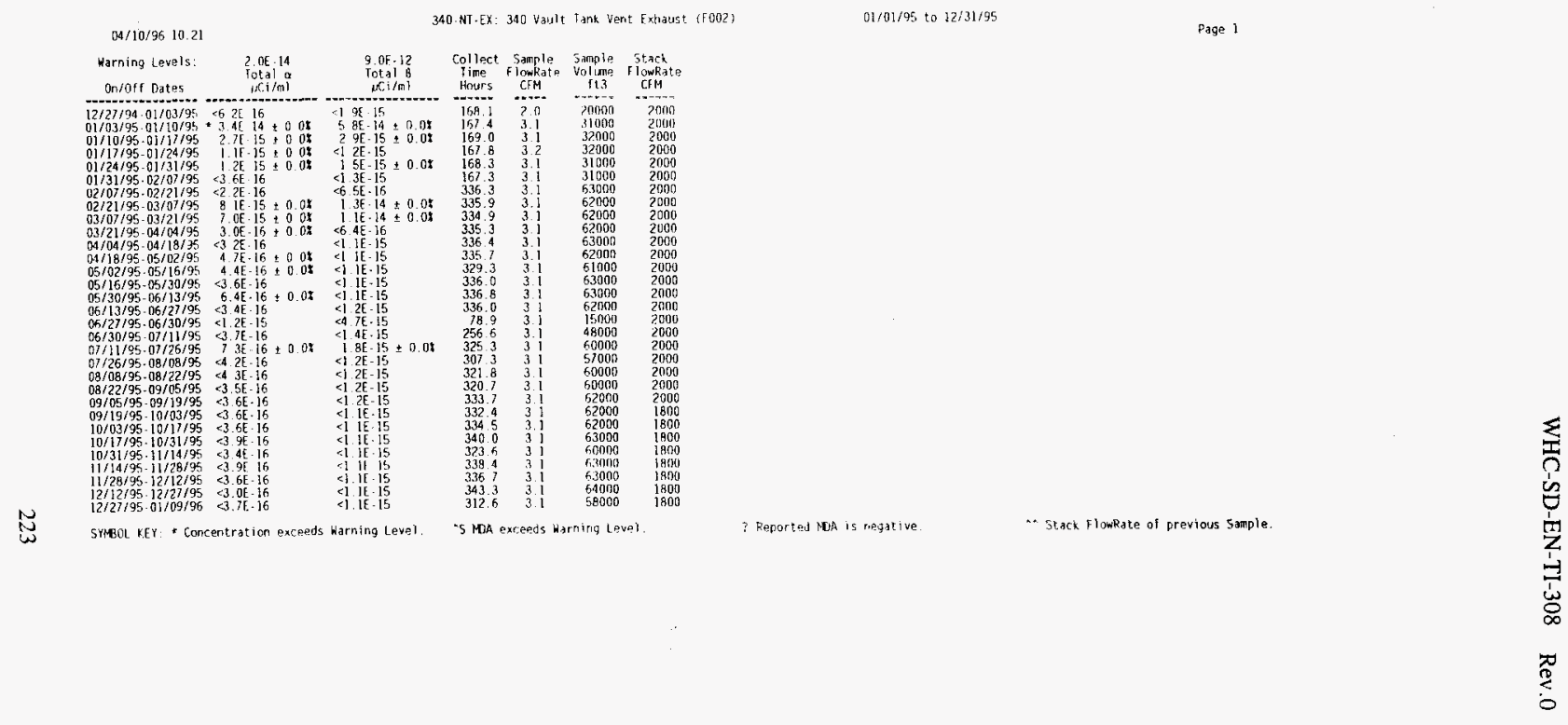


WHC-SD-EN-TI-308 Rev.0

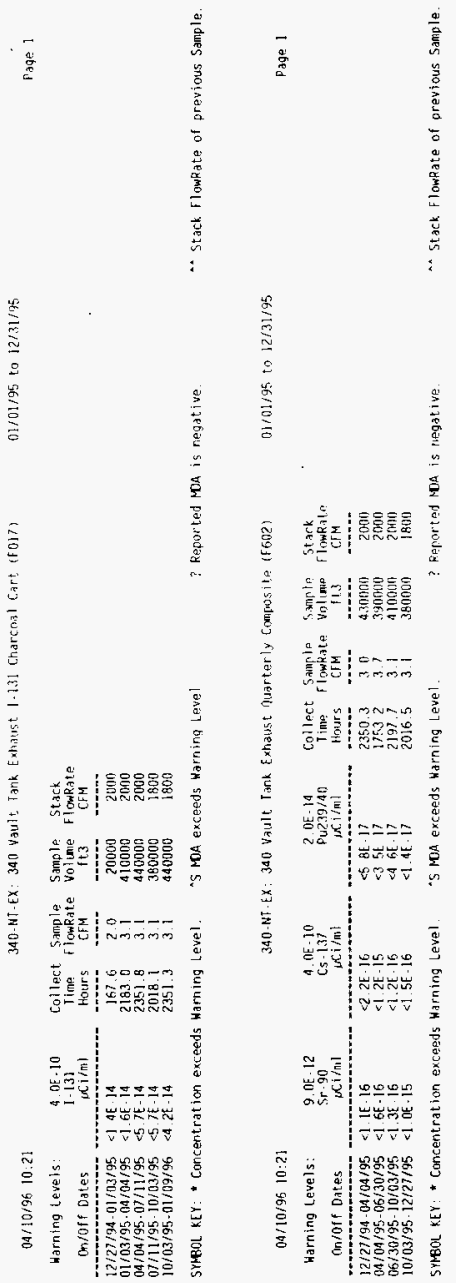


WHC-SD-EN-TI-308 Rev.0

WHC ENVIRONMENTAL RELEASE SUMMARY

$04 / 09 / 96$

340-B-BID: 340 B BUILDING (FO08)

$01 / 01 / 95$ through $12 / 31 / 95$

Total system Flow: $5.0 \mathrm{E}+09$ Liters $=1.8 \mathrm{E}+08$ Cubic Ft

The following factor(s) has (or have) been applied to the fadionuclide concentrations used in the calculation of this release to account for sampling filter efficiency, line and bend loss, and humidity. The concentrations vary inversely with the size of the factor(s).

Particulates:

Radionuclide

Total a

Total $\beta$

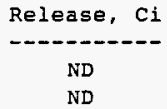

ND
0.73

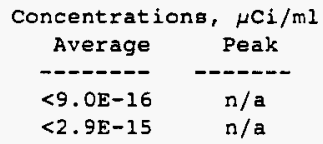


WHC-SD-EN-TI-308 Rev.0

$\vec{a}$

3
0
0
0
0
0
0

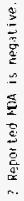

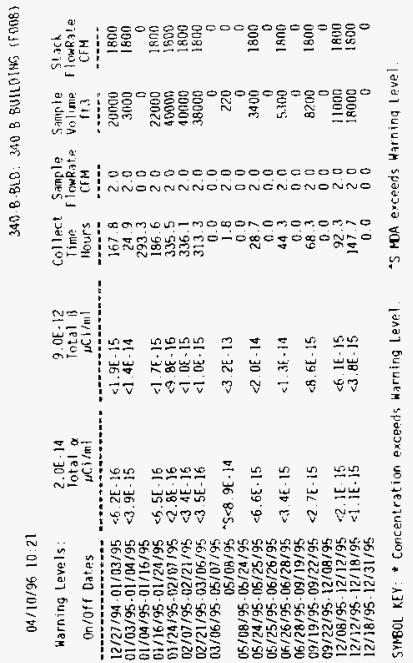


340-DECON: 340 DECONTAMINATION FACILITY (FO09)

$$
01 / 01 / 95 \text { through } 12 / 31 / 95
$$

Total system Flow: $1.1 E+11$ Liters $=3.8 \mathrm{E}+09$ Cubic $\mathrm{Ft}$

The following factor(s) has (or have) been applied to the radionuclide concentrations used in the calculation of this release to account for sampling filter efficiency, line and bend loss, and humidity. The concentrations vary inversely with the size of the factor(s).

Particulates:

Radionuclide
Total $\alpha$
Total $\beta$

Release, Ci
$-2.7 E-07$
$8.5 E-07$

0.73

$\begin{array}{cc}\begin{array}{c}\text { Concentrations, } \mu \mathrm{Ci} / \mathrm{ml} \\ \text { Average }\end{array} & \text { Peak } \\ - & ----- \\ 1.8 \mathrm{E}-15 & 2.1 \mathrm{E}-14 \\ 5.8 \mathrm{E}-15 & 5.7 \mathrm{E}-14\end{array}$




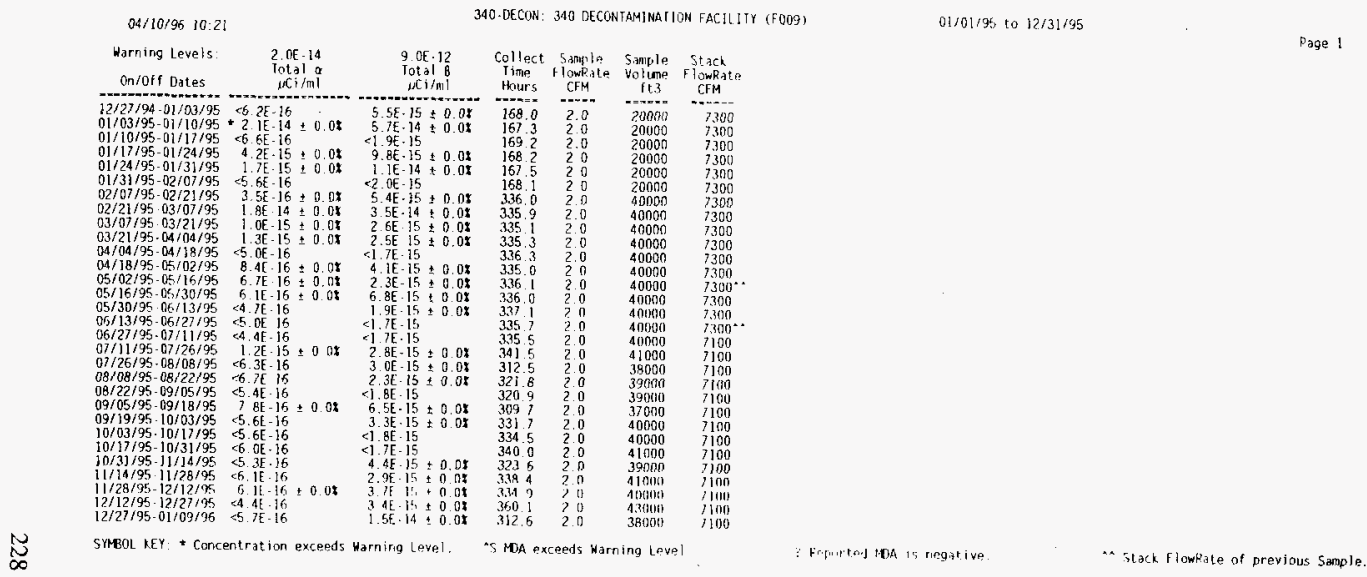




\section{WHC-SD-EN-TI-308 Rev.0}

WHC ENVIRONMENTAL RELEASE SUMMARY

$04 / 09 / 96$

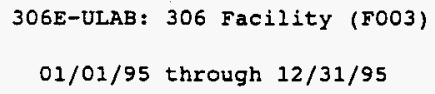

Total System Flow: $4.4 E+10$ Liters $=1.5 E+09$ Cubic Ft

The following factor(s) has (or have) been applied to the radionuclide concentrations used in the calculation of this release to account for sampling filter efficiency, line and bend loss, and humidity. The concentrations vary inversely with the size of the factor(s).

Particulates:

Radionuclide

Total a

Total $B$

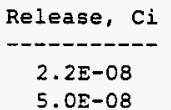

0.73

\begin{tabular}{cc}
$\begin{array}{c}\text { Concentrations, } \mu \mathrm{Cl} / \mathrm{ml} \\
\text { Average }\end{array}$ & Peak \\
\hline $3.6 \mathrm{E}-16$ & $\mathbf{8 . 6 \mathrm { E } - 1 6}$ \\
$8.4 \mathrm{E}-16$ & $1.5 \mathrm{E}-15$
\end{tabular}




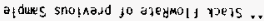

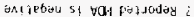

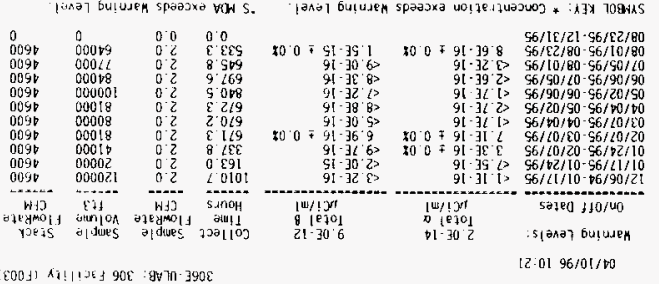


308-TRIGA: 308 E-8-9 (F006, F007)

$\star \star 01 / 01 / 95$ through $12 / 31 / 95 \star \star *$

Total system Flow: $2.7 \mathrm{E}+10$ Liters $=9.6 \mathrm{E}+08$ Cubie Ft.

The following factor(s) has (or have) been applied to the radionuclide concentrations used in the calculation of this release to account for sampling filter efficiency, line and bend loss, and humidity. The concentrations vary inversely with the eize of the factor(s).

$$
\begin{array}{ll}
\text { Particulates: } & 0.73 \\
\text { Halogens: } & 0.87
\end{array}
$$

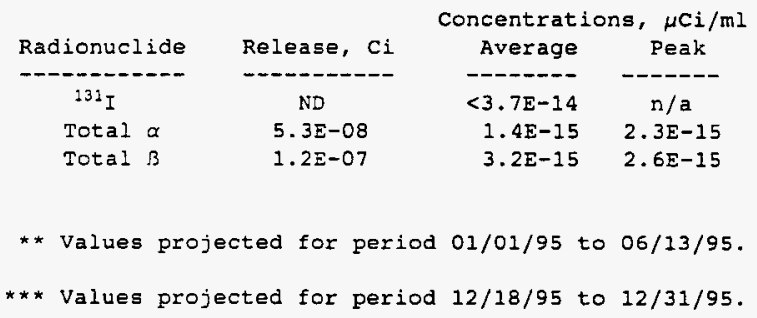




\section{WHC-SD-EN-TI-308 Rev.0}
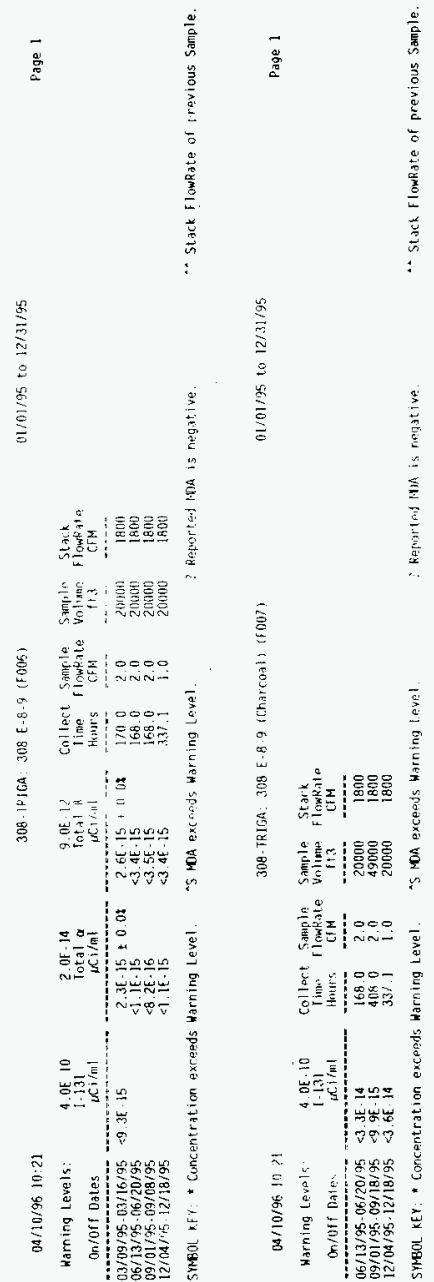
WHC-SD-EN-TI-308 Rev.0

WHC ENVIRONMENTAL RELEASE SUMMARY

377-GEL: 377 GEOTECHNICAL ENGINEERING LABORATORY (FO10)

$$
01 / 01 / 95 \text { through } 12 / 31 / 95 \star \star \star
$$

Total system Flow: $1.2 \mathrm{E}+11$ Iiters $=4.4 \mathrm{E}+09$ Cubic Ft

The following factor(s) has (or have) been applied to the radionuclide concentrations used in the calculation of this release to account for sampling filter efficiency, line and bend lose, and humidity. The concentrations vary inversely with the size of the factor $(s)$.

$$
\text { Particulates: } \quad 0.73
$$

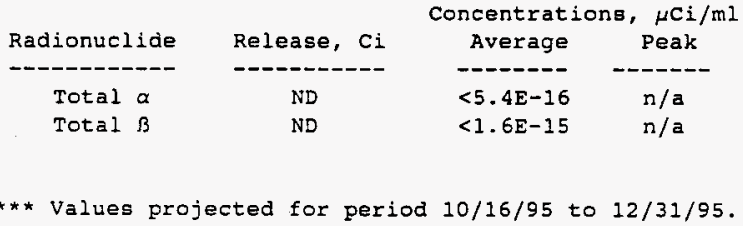




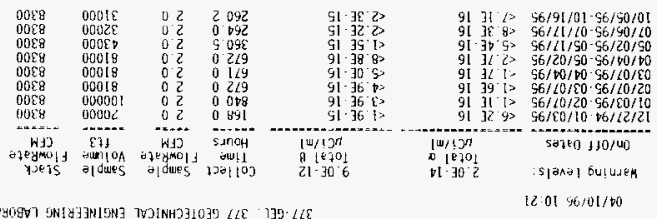


WHC-SD-EN-TI-308 Rev.0

2.7400 Area 


\section{WHC-SD-EN-TI-308 Rev.0}

WHC ENVIRONMENTAL RELEASE SUMMARY

FFIF-RESB: FFTF LOWER REACTOR SERVICE BUILDING (F012, F016)

$$
01 / 01 / 95 \text { through } 12 / 31 / 95
$$

Total System Flow: 2.7E+11 Liters $=9.7 E+09$ Cubic Ft

The following factor(s) has (or have) been applied to the radionuclide concentrations used in the calculation of this release to account for sampling filter efficiency, line and bend loss, and humidity. The concentrations vary invergely with the size of the factor (s).

$$
\begin{array}{ll}
\text { Particulates: } & 0.73 \\
\text { Halogens: } & 0.87
\end{array}
$$

Radionuclide
${ }^{131} I$
Total $\alpha$
Total $B$

$\begin{array}{cc}\text { Concentrations, } \mu \mathrm{Ci} / \mathrm{ml} \\ \text { Average } & \text { Peak } \\ ----- & ------ \\ <1.7 \mathrm{E}-14 & \mathrm{n} / \mathrm{a} \\ 2.1 \mathrm{E}-15 & 1.5 \mathrm{E}-14 \\ 8.5 \mathrm{E}-15 & 5.2 \mathrm{E}-14\end{array}$




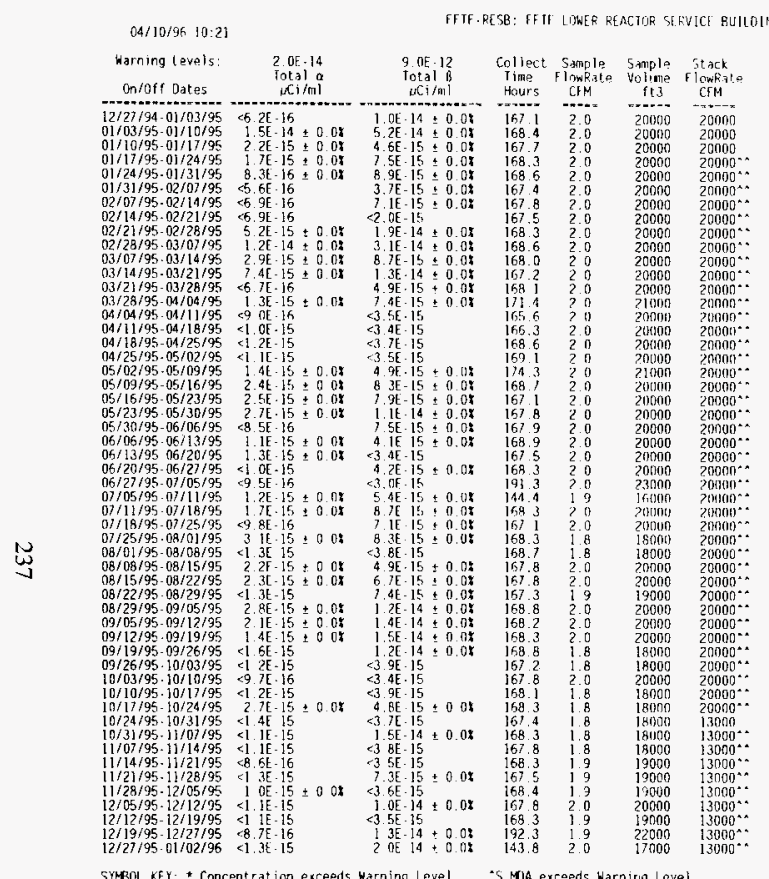

$01 / 01 / 95$ to $12 / 31 / 95$

\begin{tabular}{|c|c|c|c|c|c|c|}
\hline On/off Dates & 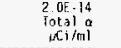 & 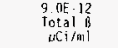 & $\begin{array}{l}\text { Collect } \\
\text { Time } \\
\text { Hours }\end{array}$ & $\begin{array}{c}\text { Samplo } \\
\text { Flowkite } \\
\text { CHM }\end{array}$ & $\begin{array}{l}\text { 5ximple } \\
\text { yolime } \\
\text { ft3 }\end{array}$ & $\begin{array}{c}5 t \text { tack } \\
\text { flowf } \\
C S M\end{array}$ \\
\hline $27 / 94-01 / n 3$ & क. $2 \mathrm{E}-16$ & $1.0 \mathrm{E} \cdot 14$ & 157.1 & 2.0 & & 20000 \\
\hline $\begin{array}{l}01 / 03 / 45-01 / 10 / 95 \\
01 / 10 / 95-01 / 1 / 796\end{array}$ & $\begin{array}{l}1.5 \mathrm{E}-14 \\
2.2 \mathrm{E}-15\end{array}$ & $\begin{array}{l}5.2 \mathrm{E}-14 \\
4 \mathrm{EE}-15\end{array}$ & 168.4 & & 20000 & 20000 \\
\hline $117 / 95.01 / 24 / 95$ & $7 E-15$ & & 158.3 & 2.0 & 20000 & $200000^{\circ}$ \\
\hline $01 / 31 / 95$ & $8.36-16 \pm 0$ & $8.96-15 \div 0.04$ & 168.5 & 0 & 20000 & $20000 \%$ \\
\hline $31 / 95.02 / 07 / 95$ & $5.6 E .16$ & 3. $76-15=0.0 \%$ & 1674 & 2.0 & & 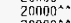 \\
\hline $\begin{array}{l}807 / 95.12 / 14 / 95 \\
/ 14 / 95.02 / 21 / 95\end{array}$ & $\begin{array}{l}6.9 E-16 \\
6.9 E-15\end{array}$ & $\begin{array}{l}7.1 \mathrm{E}-15 \\
2.0 \mathrm{E}-15\end{array}$ & 167.8 & & 200000 & $20000^{n-1}$ \\
\hline$/ 21 / 95-02 / 28 / 95$ & 5. $2 E-15=$ & $1.9 E-14 \pm 0.0 x$ & $\begin{array}{l}1685 \\
1683\end{array}$ & & & $20000^{\circ}$ \\
\hline & $2 E-14$ & $3.1 \mathrm{E}-14$ & 168.6 & & 200000 & 20000 \\
\hline $\begin{array}{l}5.03 / 4 / 95 \\
.03 / 21 / 95\end{array}$ & $\begin{array}{l}9 E-15 \pm 0.07 \\
4[-15 \pm 0.08\end{array}$ & $\begin{array}{l}\text { 8. } .16-15 \pm \\
1.3 \mathrm{E} \cdot 14 \pm\end{array}$ & $\begin{array}{l}168.0 \\
167.2\end{array}$ & ? & $\begin{array}{l}20000 \\
20000\end{array}$ & $\begin{array}{l}20000^{\circ} \\
20000^{\circ}\end{array}$ \\
\hline $\begin{array}{l}195-03 / 28 / 95 \\
195-04 / 04 / 95\end{array}$ & <6. $7[-16$ & 4. $9 \bar{E}-15+0.08$ & & 20 & 20000 & 2noun : \\
\hline $104 / 95.01 / 11 / 95$ & $=9.3 \mathrm{E}-1.5 \pm 0.08$ & $3.45-15$ & $\begin{array}{l}1718 \\
165,5 \\
6\end{array}$ & $\begin{array}{l}20 \\
20\end{array}$ & $\begin{array}{l}210000 \\
200001\end{array}$ & 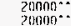 \\
\hline $11 / 95-04 / 18 / 95$ & $<.06 .15$ & 3.46 .15 & & 20 & $\begin{array}{l}2101906 \\
201908\end{array}$ & $20004 \%$ \\
\hline 195 & $\begin{array}{l}<1.2 \mathrm{E}-15 \\
<1.15 \\
<-15\end{array}$ & $\begin{array}{l}3.7 t-15 \\
35 .-15\end{array}$ & & & 2000100 & $20000^{2}:$. \\
\hline $102 / 95.05 / 109 / 95$ & $=4 t-15$ & $4.95 \cdot 15+0.138$ & Dite & & $2100 \mathrm{n}$ & 200100. \\
\hline $9 / 95-05 / 16 / 95$ & $2.4 \mathrm{E} \cdot 5$ & $83 \mathrm{E}-15$ & & & 200010 & $20000^{-2}$ \\
\hline 5.-05/23/95 & $2.5 \overline{5} \cdot 15 \pm$ & $3.96-15$ & & & 20000 & $20090 \%$ \\
\hline $5.05 / 30 / 95$ & $2.76 \cdot 15 \pm$ & 1. 16.14 & & & 20000 & $00:-2$ \\
\hline $06 / 06 / 95 \cdot 06 / 13 / 95$ & 89 & $\begin{array}{l}2.5 E-15 \div \\
4.16\end{array}$ & & & 200000 & $201000:=$ \\
\hline $06 / 13 / 9506 / 20 / 95$ & 36 & $=3.46-15$ & & & 201000 & $20090^{\circ}=$ \\
\hline $\begin{array}{l}06 / 20 / 95-06 / 27 / 95 \\
06 / 27 / 95\end{array}$ & - $.00 \cdot 15$ & $4.2 \mathrm{E}-15 \div 0.0 \mathrm{z}$ & 16 & & 20000 & 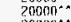 \\
\hline & $=9.5 E-16$ & $3, \pi f \cdot 15$ & & & & Poxiso:": \\
\hline & $1.7 E-65 \pm$ & $5.4 \mathrm{E}-15 \div$ & & & 20 & 7hlowin: \\
\hline$-07 / 25 / 95$ & c9.8E-16 & $7.16 \cdot 15 \div$ & $\begin{array}{ll}16,8 \\
15 .\end{array}$ & & 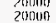 & 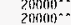 \\
\hline $0.08 / 01 / 95$ & $31 \mathrm{it}-15 \pm 0$ & B. $31 \cdot 15=$ & & & ii & $20000 \%$ \\
\hline 0 & $\begin{array}{l}135 \\
4\end{array}$ & $=3.8 \mathrm{E} \cdot 15$ & & & & \\
\hline $08 / 1$ & $15+$ & & & & & \\
\hline .06729 & c1 $3 t-15$ & $3.4 \mathrm{E}-15$ & & & & $00^{\circ}$ \\
\hline $08 / 29 / 95-09 / 05 / 95$ & $2.8 \mathrm{E}-15 \pm$ & $2-14$ & & & & \\
\hline $09 / 65 / 95-09 / 12 / 95$ & 2. IE- $15 \pm$ & 1.4 & 16 & & 20 & \\
\hline $09 / 12 / 95-09 / 19 / 95$ & $\begin{array}{r}1.4 \mathrm{E}-15 \pm \\
\mathrm{C1}-6 \mathrm{E}-15\end{array}$ & $1,5 E-14 \div 9.08$ & & & 20000 & $20000^{2}=$ \\
\hline 5.101 & $\therefore 2 \mathrm{E}-15$ & $396-15$ & & & $\begin{array}{l}18000 \\
18000\end{array}$ & $20000^{\circ}$ \\
\hline 95. 1001 & 97.16 & 34. & & & & 20 \\
\hline $10 / 10 / 95.10 / 17 / 95$ & $<1.2 \mathrm{E}-15$ & 15 & & & & (2) \\
\hline $\begin{array}{l}10 / 17 / 95-10 / 24 / 95 \\
10 / 24 / 95.10 / 31 / 95\end{array}$ & 2. $7 k-15 \pm$ & $15 \pm 0$ a & & & & $20000^{\circ}$ \\
\hline $.11 / 4$ & $\begin{array}{l}\text { 1).4E } 15 \\
<1.1 E-15\end{array}$ & 3. $5 E-15=0.08$ & & & $\begin{array}{l}1 \text { 18600 } \\
18000\end{array}$ & $\begin{array}{l}13000 \\
13000\end{array}$ \\
\hline $5.11 / 14 / 95$ & <1. $1 \mathrm{E}-15$ & $\angle B E-1$ & & & & 13 \\
\hline $11 / 14 / 95 \cdot 11 / 21 / 95$ & B. $5 \mathrm{E}-16$ & 3 & 16 & i & & $00^{\circ}$ \\
\hline & & $7.3 E-15$ & & & & $130100 \%$ \\
\hline $12 / 05$ & $\begin{array}{l}1 \\
<1.10-15-15 \\
<1.15\end{array}$ & -14 & & & 19000 & 13000 \\
\hline & & 35 & & & & $13000^{* *}$ \\
\hline $7 / 95$ & & in & & & & \\
\hline $12 / 27 / 95 \cdot 01 / 02 / 96$ & & $14+$ & & & & $13000^{\circ}$ \\
\hline
\end{tabular}

5YMen kEr: * Concentration oxcededs warning level. "S MOA exceeds Harnirig Level 
WHC-SD-EN-TI-308 Rev.0

桑

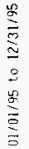

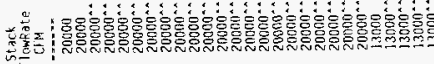

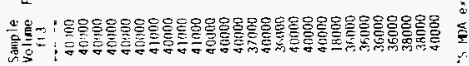

4 嵌

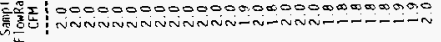

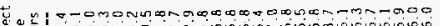

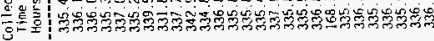
늠

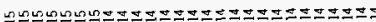

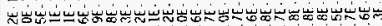

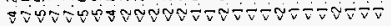

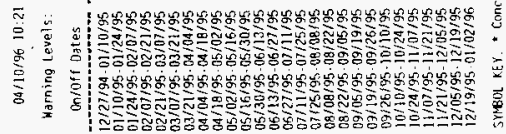




\title{
WHC-SD-EN-TI-308 Rev.0
}

WHC ENVIRONMENTAL RELEASE SUMMARY

$04 / 09 / 96$

\author{
437-MN\&ST: MASF 437-MN\&ST (FO14) \\ $01 / 01 / 95$ through $12 / 31 / 95$
}

Total System Flow: $2.1 E+11$ Liters $=7.4 E+09$ Cubic Ft

The following factor(s) has (or have) been applied to the radionuclide concentrations used in the calculation of this release to account for sampling filter efficiency, line and bend loss, and humidity. The concentrations vary inversely with the size of the factor(s).

$$
\text { Particulates: } \quad 0.73
$$

$\begin{array}{cc}\text { Radionuclide } & \text { Release, Ci } \\ \text { Total } \alpha & -2 \mathrm{E}-07 \\ \text { Total } \beta & 9.5 \mathrm{E}-07\end{array}$

$\begin{array}{cc}\text { Concentrations, } \mu \mathrm{Ci} / \mathrm{ml} \\ \text { Average } & \text { Peak } \\ - & ------ \\ 1.1 \mathrm{E}-15 & 2.1 \mathrm{E}-15 \\ 3.3 \mathrm{E}-15 & 4.2 \mathrm{E}-15\end{array}$




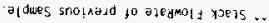

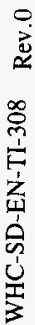

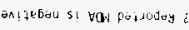

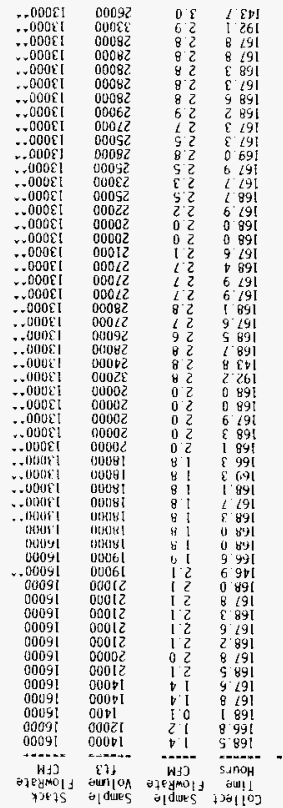

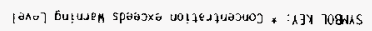

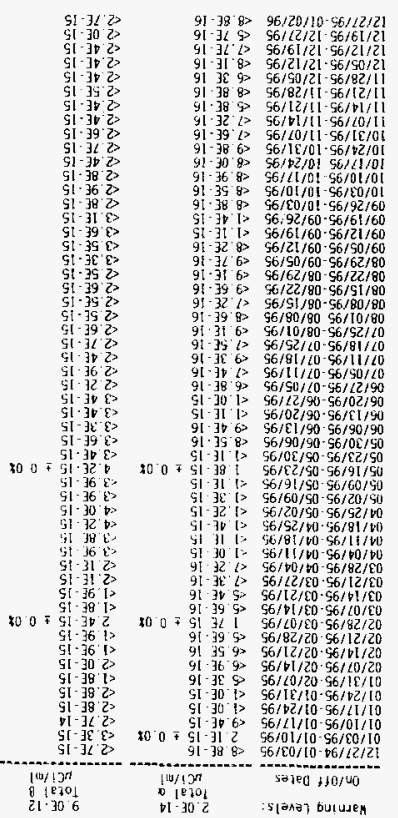

I2:01 35/01/00 


\section{WHC-SD-EN-TI-308 Rev.0}

WHC ENVIRONMENTAL RELEASE SUMMARY

FFTF-CB-E: FFTF COMBINED EXHAUST (FO11, F018, F024)

** 01/01/95 through $12 / 31 / 95 * * *$

Total System Flow: $3.2 \mathrm{E}+11$ Liters $=1.1 \mathrm{E}+10$ Cubic Ft

The following factor ( $B$ ) has (or have) been applied to the radionuclide concentrations used in the calculation of this release to account for sampling filter efficiency, line and bend loss, and humidity. The concentrations vary inversely with the size of the factor(s).

$\begin{array}{ll}\text { Particulates: } & 0.73 \\ \text { Halogens: } & 0.87 \\ \text { Others: } & 0.87\end{array}$

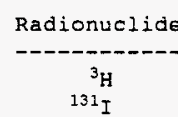

Total a

Total $B$

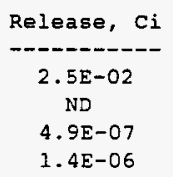

4. $9 E-07$

1. $4 \mathrm{E}-06$

$$
\begin{array}{cc}
\text { Concentrations, } \mu \mathrm{Ci} / \mathrm{ml} \\
\text { Average } & \text { Peak } \\
- & ----- \\
6.7 \mathrm{E}-11 & 9.0 \mathrm{E}-11 \\
<1.7 \mathrm{E}-14 & \mathrm{n} / \mathrm{a} \\
1.1 \mathrm{E}-15 & 3.9 \mathrm{E}-15 \\
3.3 \mathrm{E}-15 & 1.4 \mathrm{E}-14
\end{array}
$$

\footnotetext{
** Values projected for period 01/01/95 to 09/12/95.

** Values projected for period $10 / 17 / 95$ to $12 / 31 / 95$.
} 
$04 / 10 / 961021$

FFTF-CB.E. FFIT COIRINED IXHAUST (FOII

$01 / 01 / 951012131 / 95$

\begin{tabular}{|c|c|c|c|c|c|c|}
\hline $\begin{array}{l}\text { Warning Levels: } \\
\text { On/orf Dates }\end{array}$ & 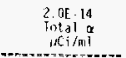 & 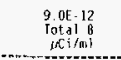 & $\begin{array}{l}\text { Collect } \\
\text { Time } \\
\text { Hours }\end{array}$ & 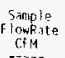 & $\begin{array}{l}\text { Saraple } \\
\text { Woligne } \\
\text { it } 3\end{array}$ & $\begin{array}{l}\text { Stack } \\
\text { FigwRate } \\
\text { CFM }\end{array}$ \\
\hline $27 / 94$ & $\sigma_{1}, 3 \mathrm{~F}=$ & $-1.95 \cdot-15$ & $10,6.8$ & I) & Pontin & याk10 \\
\hline $1095.01 / 1 / 7 / 95$ & $\begin{array}{l}1.1 E \text { L } \\
-6.6 \mathrm{E} \\
16\end{array}$ & $\begin{array}{l}<2.0 \mathrm{E} \cdot 15 \\
<\mathrm{l} .9 \mathrm{E} \cdot 15\end{array}$ & $\begin{array}{l}168.5 \\
157.7\end{array}$ & ? & 200000 & (2416019 \\
\hline $17 / 95-61 / 24 / 95$ & $\Rightarrow \cdot 2 E \cdot 16$ & $<9 E-15$ & 1692 & $\begin{array}{l}2.0 \\
2.0\end{array}$ & 20000 & $\begin{array}{l}4000 \\
4000\end{array}$ \\
\hline $1 / 31 /$ & $\begin{array}{l}7.2 E \cdot 16 \\
<5.7 E-16\end{array}$ & $\begin{array}{l}<.9 \mathrm{E}-15 \\
2.0 \mathrm{E}-15\end{array}$ & 1703 & 2.0 & 20000 & 24000 \\
\hline $02 t$ & $<6.9 E-16$ & $\begin{array}{l}2.0 E-15 \\
<2.0 E-15\end{array}$ & $\begin{array}{l}165.8 \\
167.9\end{array}$ & 2.13 & $\begin{array}{l}20000 \\
20000\end{array}$ & $\begin{array}{l}24000 \\
240000\end{array}$ \\
\hline $\begin{array}{l}4 / 95.02 / 21 / 95 \\
1 / 95.02 / 28 / 95\end{array}$ & $\begin{array}{l}<6.9 \mathrm{E} \cdot 15 \\
6.3 \mathrm{~F} \\
\mathrm{~d}\end{array}$ & $\begin{array}{r}2.0 \mathrm{E} \cdot 15 \\
2.0 \mathrm{~F} \cdot 15\end{array}$ & 168.3 & 20 - & & $\begin{array}{l}240010 \\
24010\end{array}$ \\
\hline $\begin{array}{l}8 / 95-03 / 67 / 95 \\
8 / 9503 / 4 / 95\end{array}$ & $3.9 \mathrm{E}-15 \pm 0.04$ & $6.96-15 \pm 0.0 x$ & $\begin{array}{l}167.6 \\
168.7\end{array}$ & $\begin{array}{l}2.0 \\
2.0\end{array}$ & $\begin{array}{l}20000 \\
20000\end{array}$ & $\begin{array}{l}24000 \\
24000\end{array}$ \\
\hline $\begin{array}{l}07 / 95.03 / 14 / 95 \\
11 / 95.03 / 21 / 95\end{array}$ & $\begin{array}{l}\text { 5. } 9 \mathrm{E} \cdot 16 \\
1.4 \mathrm{E} \cdot 15+0.0 \mathrm{t}\end{array}$ & $\begin{array}{l}<1.9 E-15 \\
<2.0 E-15\end{array}$ & 156.2 & 20 & 20000 & 24000 \\
\hline $\begin{array}{l}3 / 21 / 95.03 / 28 / 95 \\
3 / 28 / 9.04 / 04 / 95\end{array}$ & $66.7 \mathrm{E}-16$ & 2.0. 015 & 168,0 & & $\begin{array}{l}20000 \\
20000\end{array}$ & $\begin{array}{l}24000 \\
24000\end{array}$ \\
\hline (1) & 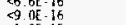 & $\begin{array}{l}-1.9 \mathrm{gE} \cdot 15 \\
-3.5 \mathrm{E}-15\end{array}$ & $\begin{array}{l}171.5 \\
165.1\end{array}$ & 2 & 21000 & 24000 \\
\hline & $<106.15$ & a. $45-15$ & $\begin{array}{l}165.7 \\
166.3\end{array}$ & 20 & $\begin{array}{l}20000 \\
270000\end{array}$ & $\begin{array}{l}24000 \\
24000\end{array}$ \\
\hline 5 & $\begin{array}{l}<12 E-15 \\
\qquad 1.1 E-15\end{array}$ & $\begin{array}{l}37 . \cdot 15 \\
<3.58-15\end{array}$ & 168.8 & $\begin{array}{l}20 \\
20\end{array}$ & 20190 & 24000 \\
\hline 95 & d. 15.15 & $3.48-15$ & 174.3 & 2.8 & $\begin{array}{l}20000 \\
20000\end{array}$ & $\begin{array}{l}24000 \\
24000\end{array}$ \\
\hline $05 / 16 / 95-05$ & $\begin{array}{l}<9.75 \cdot 16 \\
<725 \cdot 15\end{array}$ & $\begin{array}{l}35 \bar{E}-15 \\
<3 \quad 2-15\end{array}$ & & 2. & 20000 & $24000 \%$ \\
\hline $5 / 23 / 9$ & द. IE 15 & $<3.4 E-15$ & & $\frac{2}{2}$ & 000 & 24000 \\
\hline 95 & $\begin{array}{l}88.5 E-16 \\
94.16\end{array}$ & $<3.6 \mathrm{E}-15$ & 16 & 2 & 100 & $\begin{array}{l}24000 \\
24000\end{array}$ \\
\hline 35 & $\Leftrightarrow 1 \mathrm{lt} \cdot 15$ & $\begin{array}{l}3.3 E-15 \\
3.4 E-15\end{array}$ & & $?$ & 00 & 24000 \\
\hline & $\angle 10 \mathrm{OE} 15$ & $34 \mathrm{E} .15$ & $\begin{array}{l}16 \\
16 \\
-16\end{array}$ & $\frac{2}{2}$ & 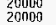 & 200000. \\
\hline $5 / 9$ & 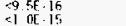 & 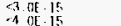 & & $?$ & 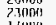 & $100^{\circ}{ }^{\circ}$ \\
\hline 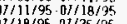 & $<13 \mathrm{E}$ & 345.15 & min & $\xi$ & & 7 \\
\hline & $\begin{array}{l}<9 \mathrm{gE} \cdot 16 \\
-1.6 \mathrm{E} \cdot 15\end{array}$ & $\begin{array}{l}3.5 E \cdot 15 \\
\times 4.6 E-15\end{array}$ & & $?$ & & (iT) \\
\hline & $\begin{array}{l}1.06 .15 \\
<1.15\end{array}$ & $<38 \mathrm{BE} \cdot 15$ & & I. & & \\
\hline & $<.1 E .15$ & $\begin{array}{rl}3 & \text { OLE } \\
3 & 15 \\
\end{array}$ & & i. & 10 & $000^{\circ}$ \\
\hline & $\begin{array}{l}1.4 \mathrm{E} \\
3.15 \mathrm{BE} \cdot 15\end{array}$ & $\begin{array}{l}9 E \cdot 15 \\
4 \mathrm{E}-15\end{array}$ & 168 & i. & 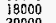 & \\
\hline & $<1.0 \mathrm{E}-15$ & $\begin{array}{r}3.45-15 \\
3.5 E \cdot 15\end{array}$ & & 2.5 & & \\
\hline $89 / 12$ & $1.4 E \cdot 15=$ & <.5E-15 & & c. & $\frac{00}{00}$ & $0000^{*}$ \\
\hline 09 & $\begin{array}{l}1.15 \\
<1.15 \\
<1.15\end{array}$ & $\begin{array}{l}<3.6 \mathrm{E} \\
<3 \mathrm{E}\end{array}$ & 168 & 2. & 20000 & \\
\hline & $<1.1 \mathrm{E} \cdot 15$ & $\begin{array}{l}15 \\
15\end{array}$ & $\begin{array}{l}16.2 \\
16 .\end{array}$ & & 20010 & \\
\hline & $<9.7 \mathrm{E}$ & & 167 & & & \\
\hline$D / / 2+2$ & 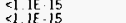 & $\begin{array}{l}15 \\
15\end{array}$ & & & & \\
\hline & & & & & & (108) \\
\hline & & & & & & \\
\hline & 15 & & & & & $00^{* * *}$ \\
\hline & $<8.2 E \cdot 16$ & 4 & & & & \\
\hline $121 /$ & & & & $?$ & & \\
\hline 105 & & 34 & 168 & 2. & $\begin{array}{l}20000 \\
20000\end{array}$ & 26000 \\
\hline $2 / 12$ & & 3 & & & & 200 \\
\hline $1 / 19 / 9$ & 5 & 5 & & & & \\
\hline & & & & & 17000 & 0000 \\
\hline
\end{tabular}

sheoL KEY: * Concentration exceeds Karning level 
C4/10/95 10:21

\begin{tabular}{|c|c|}
\hline $\begin{array}{l}\text { Warning levels: } \\
\text { on/off bates }\end{array}$ & $\begin{array}{l}40[-10 \\
1 .|3| \\
|C, i / m|\end{array}$ \\
\hline 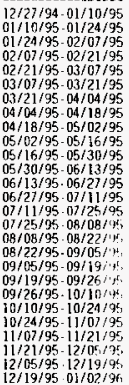 & 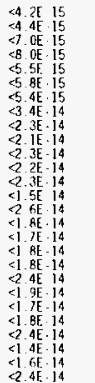 \\
\hline
\end{tabular}

CFM
CFM
2.0
2.0
2.0
2.0
2.0
2.0
2.0
2.0
2.0
2.0
2.0
2.0
2.0
2.0
1.0
1.9
2.0
2.0
2.0
2.0
2.0
2.0
2.0
2.0
1.9
2.0

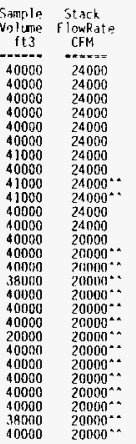

SrMeO KEY: Concentration exceeds Yarning Level

5 MDA excepds Harning Lovel

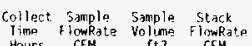

\begin{tabular}{|c|c|c|c|c|c|}
\hline $\begin{array}{l}\text { Harning levels: } \\
\text { on/off Dates }\end{array}$ & 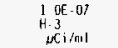 & $\begin{array}{l}\text { Collect. } \\
\text { Time } \\
\text { Hours }\end{array}$ & $\begin{array}{c}\text { Sampla } \\
\text { Foutfate } \\
\text { CFM }\end{array}$ & $\begin{array}{l}\text { Sample } \\
\text { volume } \\
\text { ft3 }\end{array}$ & $\begin{array}{l}\text { Stack } \\
\text { Flowfate } \\
\text { CFM }\end{array}$ \\
\hline $0 / 03 / 95 \cdot 10 t 1$ & \&. & & & 0 & \\
\hline
\end{tabular}

SYMOL KEY: Concentration exceeds harning Level.

¿5 MDA exceeds Warning Level. 
WHC-SD-EN-TI-308 Rev.0

WHC ENVIRONMENTAL RELEASE SUMMARY

$04 / 09 / 96$

FFTF-HT-T: FFTF REACTOR HEAT TRANSPORT SYSTEM (FO13)

$01 / 01 / 95$ through $12 / 31 / 95$

Total syatem Flow: $6.7 \mathrm{E}+10$ Literg $=2.4 \mathrm{E}+09$ Cubic $\mathrm{Ft}$

The following factor(s) has (or have) been applied to the radionuclide concentrations used in the calculation of this release to account for sampling filter efficiency, line and bend loss, and humidity. The concentrations vary inversely with the size of the factor(s).

$$
\text { Particulates: } \quad 0.73
$$

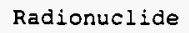

------------

Total $\alpha$

Total $\beta$

Release, Ci
$1.1 E-07$
$3.5 E-07$

$\begin{array}{cc}\begin{array}{c}\text { Concentrations, } \mu \mathrm{Ci} / \mathrm{ml} \\ \text { Average }\end{array} & \text { Peak } \\ - & ----- \\ 1.2 \mathrm{E}-15 & 6.3 \mathrm{E}-15 \\ 3.8 \mathrm{E}-15 & 1.7 \mathrm{E}-14\end{array}$




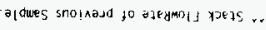
这

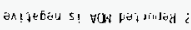

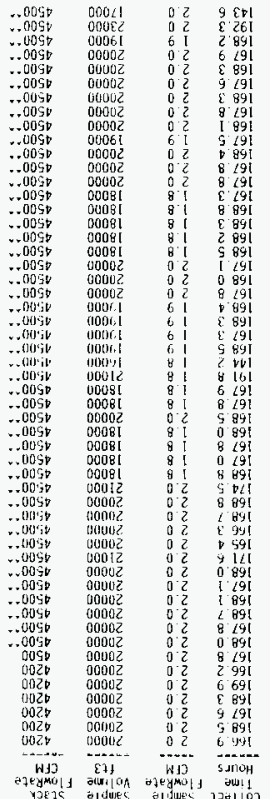

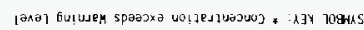

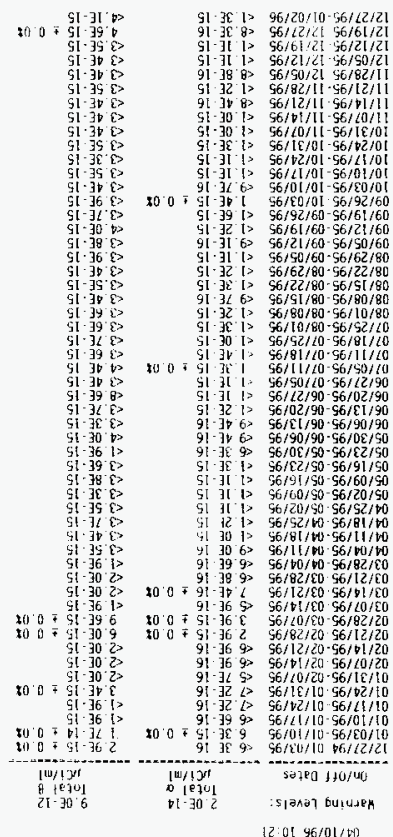


WHC-SD-EN-TI-308 Rev.0

$04 / 09 / 96$

WHC ENVIRONMENTAL RELEASE SUMMARY

$$
\begin{aligned}
& \text { 437-1-61: MASF 437-1-61 (F019) } \\
& \text { ** 01/01/95 through } 12 / 31 / 95 \star * *
\end{aligned}
$$

Total system Flow: $2.4 E+11$ Liters $=8.4 \mathrm{E}+09$ Cubic Ft

The following factor(s) has (or have) been applied to the radionuclide concentrations used in the calculation of this release to account for sampling filter efficiency, line and bend losg, and humidity. The concentrations vary inversely with the size of the factor $(s)$.

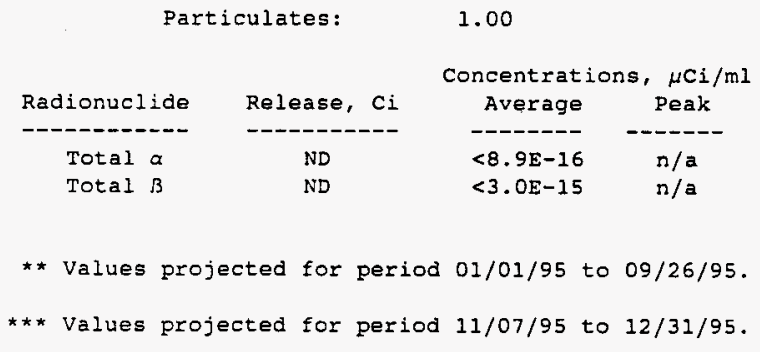


WHC-SD-EN-TI-308 Rev.0

$\bar{\square}$

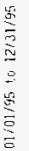

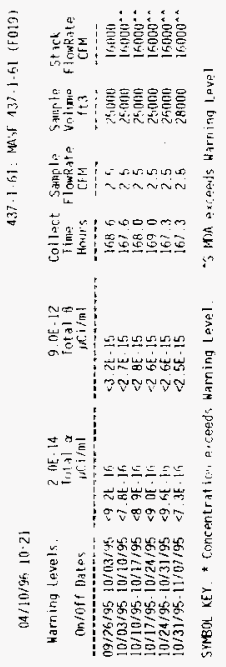


WHC-SD-EN-TI-308 Rev.0

This page intentionally left blank. 


\subsection{LIQUID EFFLUENT RELEASES \& DATA}

This section contains all of the radioactive liquid effluent release reports and the sample collection and analysis supporting data. Sub-section 3.1 contains the liquid effluent releases calculated for each major operating area, with active source terms. The remaining sub-sections include the sample collection and analysis supporting data (trend reports) and radioactive liquid effluent releases (release reports) for individual discharge points or source terms. No release or trend reports were included for those discharge points or source terms which did not operate in CY-1995. 
WHC-SD-EN-TI-308 Rev,0

This page intentionally left blank. 
WHC-SD-EN-TI-308 Rev.0

3.1 All Area Liquid Release Reports 


\section{WHC-SD-EN-TI-308 Rev.0}

WHC ENVIRONMENTAL RELEASE SUMMARY

$04 / 01 / 96$

Radionuclides Discharged to the Columbia River from 100 Areas

$$
01 / 01 / 95 \text { through } 12 / 31 / 95 * \star *
$$

\begin{tabular}{ccc} 
Total System Flow: $1.6 \mathrm{E}+09$ Liters & $=4.3 \mathrm{E}+0 \mathrm{~B} \mathrm{Gallons}$ \\
Radionuclide & Halflife & Release, Ci \\
\hline${ }^{3} \mathrm{H}$ & 12.3 Years & $1.5 \mathrm{E}-01$ \\
${ }^{60} \mathrm{Co}$ & 5.27 Years & $1.1 \mathrm{E}-04$ \\
${ }^{90} \mathrm{Sr}$ & 29.12 Years & $2.1 \mathrm{E}-01$ \\
${ }^{106} \mathrm{Ru}$ & 368 Days & $\mathrm{ND}$ \\
${ }^{125} \mathrm{Sb}$ & 2.8 Years & $2.7 \mathrm{E}-04$ \\
${ }^{134} \mathrm{Cs}$ & 2.1 Years & $\mathrm{ND}$ \\
${ }^{137} \mathrm{Cs}$ & 30 Years & $2.1 \mathrm{E}-03$ \\
${ }^{154} \mathrm{Eu}$ & 8.8 Years & $4.1 \mathrm{E}-03$ \\
$155 \mathrm{Eu}$ & 4.96 Years & $\mathrm{ND}$ \\
$238 \mathrm{Pu}$ & 87.8 Years & $\mathrm{ND}$ \\
$239 / 240 \mathrm{Pu}$ & 24.131 Years* & $4.3 \mathrm{E}-07$ \\
$241 \mathrm{Am}$ & 432 Years & $1.2 \mathrm{E}-05$
\end{tabular}

* The halflife shown for Total Uranium is the halflife of ${ }^{238} \mathrm{U}$. The halflife shown for mixed ${ }^{239 / 240} \mathrm{Pu}$ is the halflife of ${ }^{239} \mathrm{Pu}$.

*** Values for one or more files projected forward to cover report period. 
WHC-SD-EN-TI-308 Rev.0

WHC ENVIRONMENTAL RELEASE SUMMARY

$04 / 01 / 96$

Radionuclides Discharged to Ground from 200 Areas

** $01 / 01 / 95$ through $12 / 31 / 95$

Total System Flow: $4.9 E+09$ Liters $=1.3 E+09$ Gallons

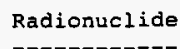

${ }^{3} \mathrm{H}$

${ }^{60} \mathrm{Co}$

${ }^{90} \mathrm{Sr}$

${ }^{99} \mathrm{TC}$

${ }^{103} \mathrm{Ru}$

${ }^{206} \mathrm{Ru}$

$123 \mathrm{sn}$

$125 \mathrm{Sb}$

${ }^{134} \mathrm{Cs}$

${ }^{137} \mathrm{Cs}$

${ }^{147} \mathrm{Pm}$

${ }^{252 \mathrm{EU}}$

${ }^{154} \mathrm{Eu}$

$155_{\mathrm{Eu}}$

Total U

${ }^{238} \mathrm{Pu}$

$239 / 240_{\mathrm{PU}}$

${ }^{24}$ Am

Halflife
12.3 Years
5.27 Years
29.12 Years
$2.13 \mathrm{E}+5$ Years
39 Days
368 Days
115 Days
2.8 Years
2.1 Years
30 Years
2.6 Years
13.3 Years
8.8 Years
4.96 Years
$4.5 E+9$ Years*
87.8 Years
24.131 Years*
432 Years

Release, $\mathrm{Ci}$

1. $5 \mathrm{E}-02$

9. 5E-03

1. 2E-01

2. $3 E-04$

ND

1. $9 E-01$

1. $\mathrm{OE}-02$

3. $8 \mathrm{E}-03$

1. $2 \mathrm{E}-03$

4. 5E-02

ND

1. $1 \mathrm{E}-03$

3. $3 \mathrm{E}-02$

2. $3 \mathbf{E}-02$

5. 5E-04

7. 5E-04

7. 2E-03

1. $2 E-03$

* The halflife shown for Total Uranium is the halflife of ${ }^{238} \mathrm{U}$. The halflife shown for mixed $239 / 240 \mathrm{Pu}$ is the halfife of ${ }^{239} \mathrm{Pu}$.

* Values for one or more files projected backward to cover report period. 
WHC-SD-EN-TI-308 Rev.0

This page intentionally left blank. 
WHC-SD-EN-TI-308 Rev.0

3.2 100 Areas 
WHC-SD-EN-TI-308 Rev.0

WHC ENVIRONMENTAL RELEASE SUMMARY

\author{
1LK004: 1908-K OUTFALI (Y130) \\ $01 / 01 / 95$ through $12 / 31 / 95$
}

Total System Flow: $1.6 E+09$ Iiters $=4.3 E+08$ Gallons

Radionuclice
${ }^{30} \mathrm{H}$
${ }^{60} \mathrm{Co}$
${ }^{90} \mathrm{Sr}$
$206 \mathrm{Ru}$
$225 \mathrm{Sb}$
${ }^{134} \mathrm{Cs}$
$237 \mathrm{Cs}$
$254 \mathrm{Eu}$
$=55 \mathrm{Eu}$
$238 \mathrm{Pu}$
$239 / 24 \mathrm{Pu}$
$245 \mathrm{Am}$
Total $\alpha$
Total B

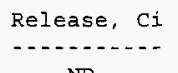

Concentrations, $\mu \mathrm{Ci} / \mathrm{ml}$

$\begin{array}{rc}\text { Average } & \text { Peak } \\ -4.3 E-08 & \text { n/a } \\ 1.3 E-07 & 3.0 E-07 \\ -4.0 E-10 & 4.3 E-09 \\ 3.0 E-10 & 5.3 E-09 \\ -2.5 E-09 & 1.9 E-08 \\ -1.9 E-10 & 4.0 E-09 \\ -1.0 E-11 & 4.9 E-09 \\ 3.4 E-10 & 8.8 E-09 \\ 2.4 E-09 & 1.2 E-08 \\ -1.1 E-09 & 4.7 E-09 \\ 3.4 E-12 & 2.7 E-10 \\ 4.9 E-13 & 1.6 E-12 \\ 7.5 E-12 & 1.7 E-11 \\ 2.2 E-10 & 8.5 E-10 \\ 7.7 E-09 & 2.2 E-08\end{array}$




\section{WHC-SD-EN-TI-308 Rev.0}

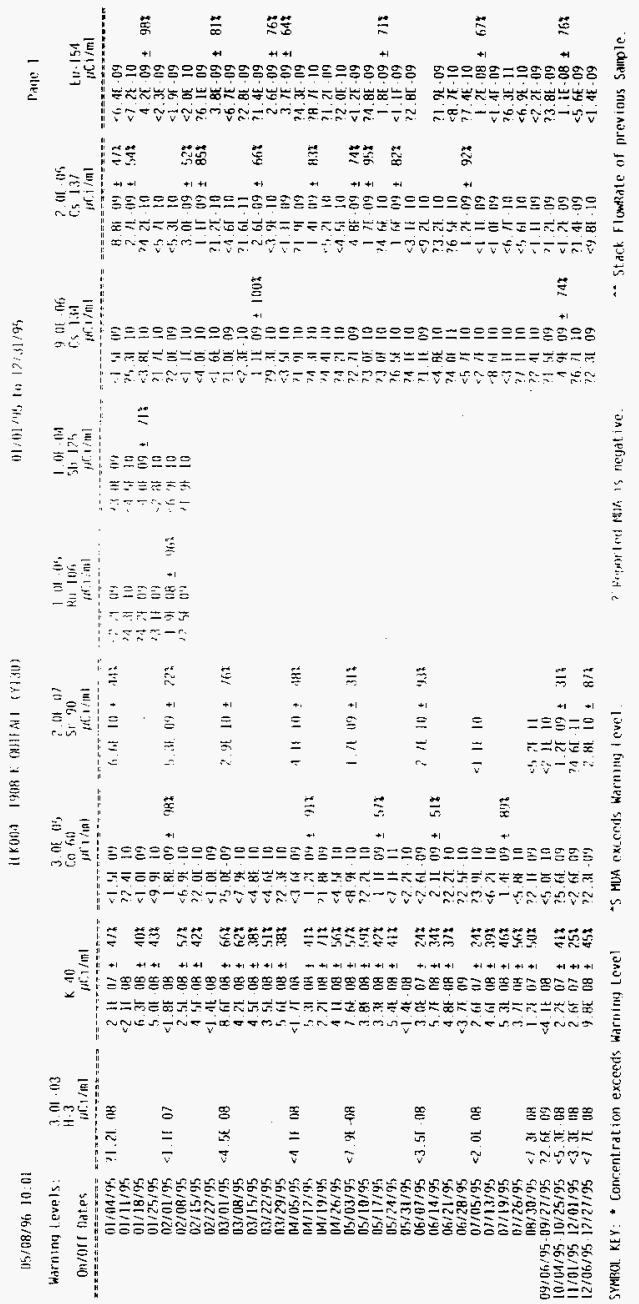


WHC-SD-EN-TI-308 Rev.0

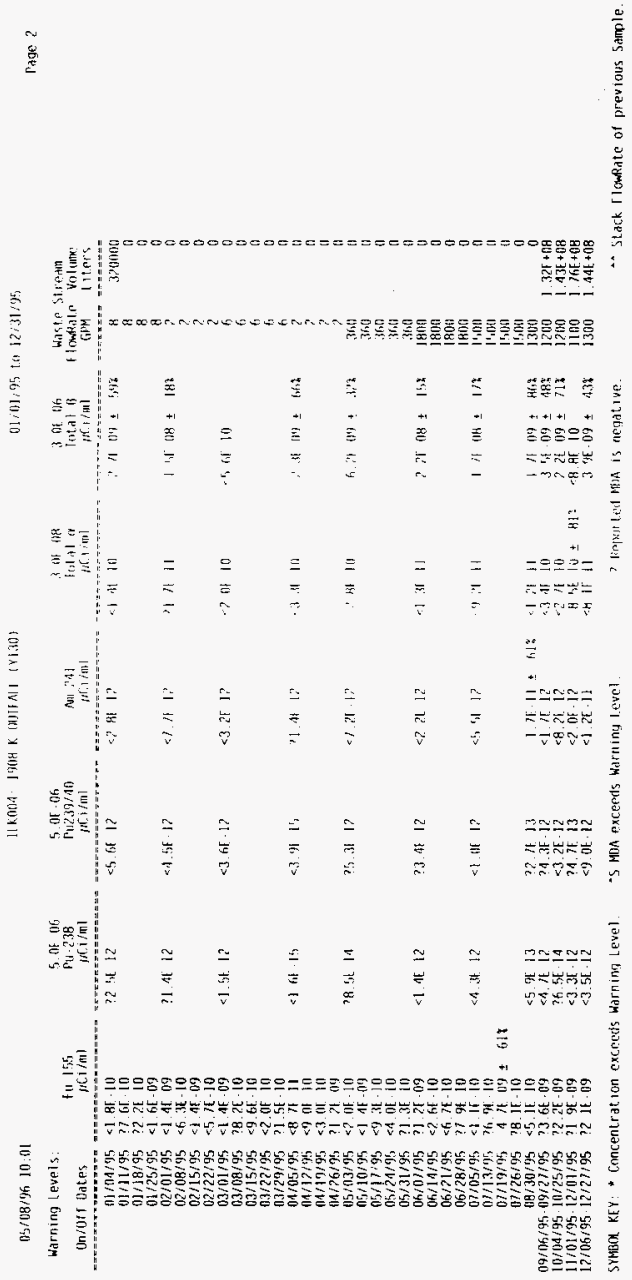


WHC-SD-EN-TI-308 Rev.0

WHC ENVIRONMENTAL RELEASE SUMMARY

$05 / 08 / 96$

1LN130: 100-N AREA RIVER SPRINGS (Y101) WELL N-46 (Y101)

$01 / 01 / 95$ through $12 / 31 / 95 * \star \star$

Total System Flow: $2.0 E+07$ Liters $=5.3 E+06$ Gallons

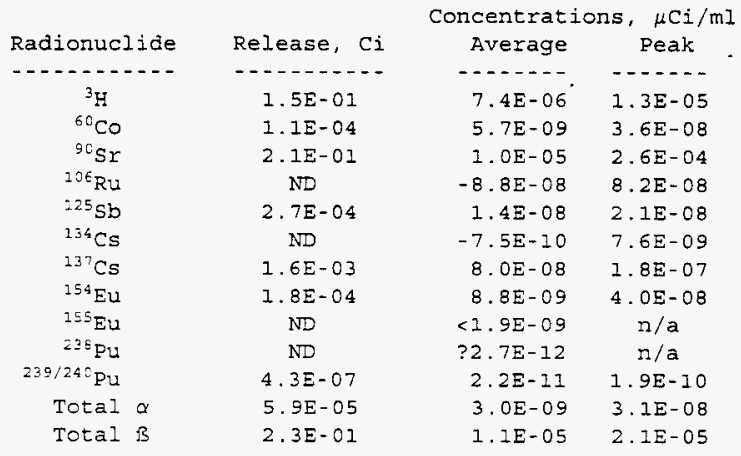

\footnotetext{
*** Values projected for period 09/06/95 to 12/31/95.

? A question mark ("?") indicates an average concentration less than a negative value.
} 


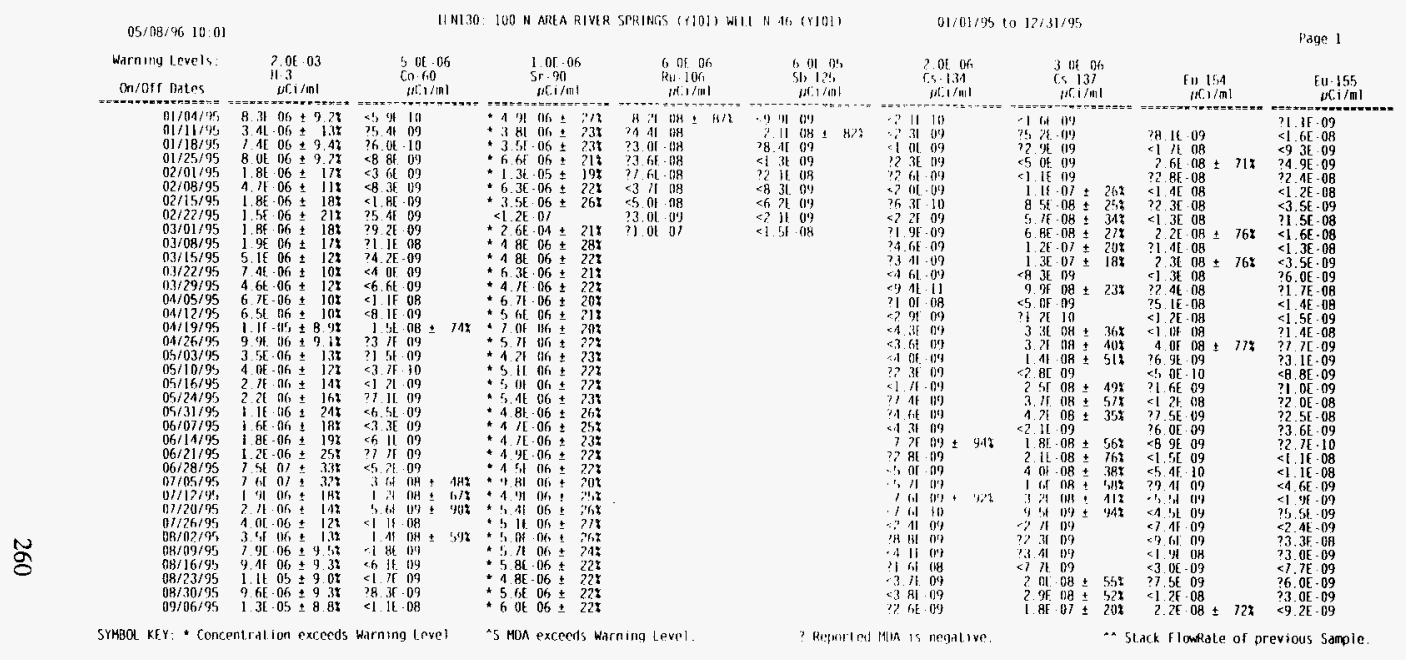




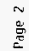

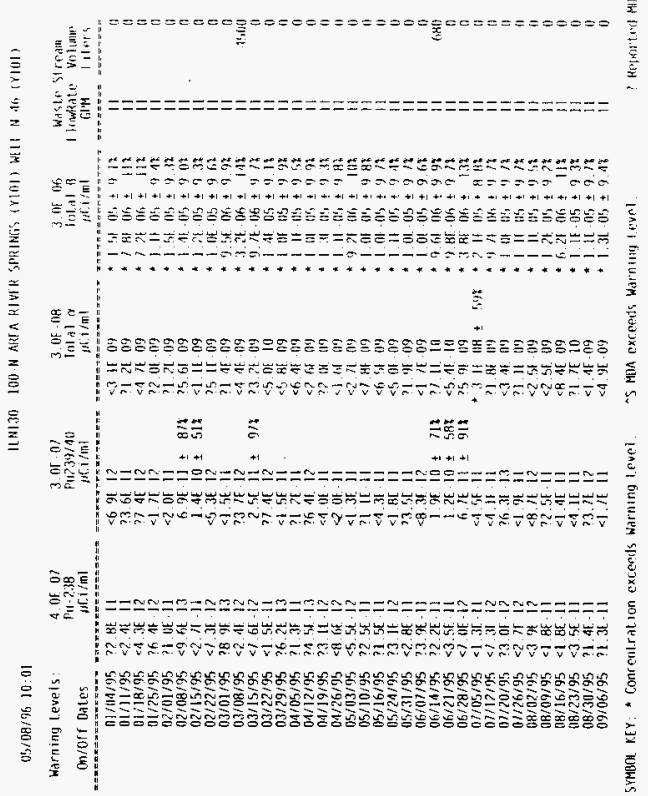


WHC-SD-EN-TI-308 Rev.0

This page intentionally left blank. 
WHC-SD-EN-TI-308 Rev.0

3.3200 East Area 
WHC-SD-EN-TI-308 Rev.0

WHC ENVIRONMENTAL RELEASE SUMMARY

$03 / 23 / 96$

$10: 11$

ACW: 242-A EVAPORATOR COOLING WATER (H108)

$01 / 01 / 95$ through $12 / 31 / 95$

Total System Flow: $7.8 E+08$ Liters $=2.1 E+08$ Gallons

\begin{tabular}{|c|c|c|c|}
\hline \multirow{2}{*}{ Radionuclide } & & \multicolumn{2}{|c|}{ Concentrations, $\mu \mathrm{Ci} / \mathrm{ml}$} \\
\hline & Release, Ci & Average & Peak \\
\hline--- & 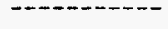 & ------- & ------- \\
\hline${ }^{3} \mathrm{H}$ & ND & $<4.5 E-07$ & $\mathrm{n} / \mathrm{a}$ \\
\hline${ }^{60} \mathrm{Co}$ & ND & $<4.0 E-09$ & $\mathrm{n} / \mathrm{a}$ \\
\hline${ }^{90} \mathrm{sr}$ & ND & $-5.9 E-09$ & $1.4 \mathrm{E}-09$ \\
\hline${ }^{106} \mathrm{Ru}$ & ND & $<4.0 E-08$ & $n / a$ \\
\hline${ }^{113} \mathrm{sn}$ & ND & $<5.0 \mathrm{E}-09$ & $n / a$ \\
\hline${ }^{137} \mathrm{Cs}$ & ND & $<4.6 \mathrm{E}-09$ & $\mathrm{n} / \mathrm{a}$ \\
\hline Total U & $4.2 E-04$ & 5. $3 E-10$ & $5.8 \mathrm{E}-10$ \\
\hline $235 \mathrm{Pu}$ & $9.7 E-06$ & $1.2 E-11$ & $1.3 E-11$ \\
\hline $239 / 240 \mathrm{Pu}$ & ND & $-7.9 E-11$ & $1.5 E-11$ \\
\hline${ }^{241} \mathrm{Am}$ & $6.3 E-05$ & $8.1 E-11$ & 1. $1 \mathrm{E}-10$ \\
\hline Total a & $3.4 \mathrm{E}-04$ & $4.3 E-10$ & $6.9 \mathrm{E}-10$ \\
\hline Total $\beta$ & $4.0 E-04$ & 5. $1 E-10$ & $5.8 \mathrm{E}-10$ \\
\hline
\end{tabular}


WHC-SD-EN-TI-308 Rev.0

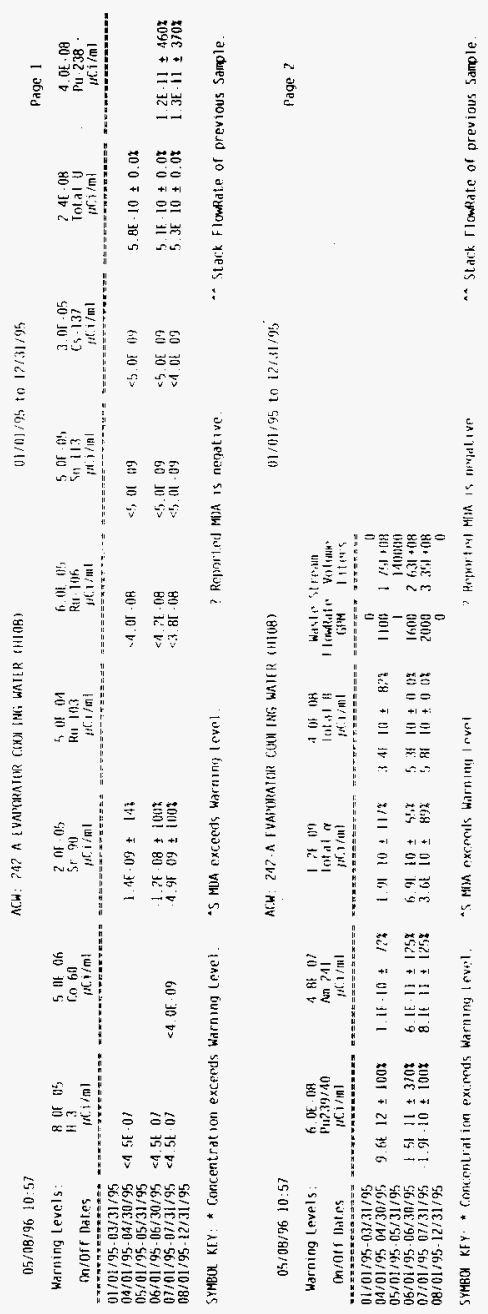


WHC ENVIRONMENTAL RELEASE SUMMARY

ASC: 242-A EVAPORATOR STEAM CONDENSATE (H110)

$01 / 01 / 95$ through $12 / 31 / 95$

Total system Flow: $2.3 E+07$ Liters $=6.0 E+06$ Gallons

Radionuclide
${ }^{60} \mathrm{H}$
${ }^{90} \mathrm{Co}$
${ }^{106} \mathrm{Sr}$
${ }^{123} \mathrm{Su}$
${ }^{137} \mathrm{Cs}$
${ }^{238} \mathrm{Pu}$
$239 / 2{ }^{\circ} \mathrm{Pu}$
${ }^{241} \mathrm{Am}$
Total $\alpha$
Total $B$

Release, Ci
$1.5 \mathrm{E}-02$
ND
ND
ND
ND
ND
$2.2 \mathrm{E}-06$
$1.7 \mathrm{E}-06$
$2.0 \mathrm{E}-06$
$2.0 \mathrm{E}-06$
$7.9 \mathrm{E}-06$

\begin{tabular}{cc} 
Concentrations, $\mu \mathrm{Ci} / \mathrm{ml}$ \\
Average & Peak \\
\hline$-6.6 \mathrm{E}-07$ & $8.9 \mathrm{E}-07$ \\
$<4.0 \mathrm{E}-09$ & $\mathrm{n} / \mathrm{a}$ \\
$-6.1 \mathrm{E}-09$ & $2.3 \mathrm{E}-09$ \\
$<6.3 \mathrm{E}-08$ & $\mathrm{n} / \mathrm{a}$ \\
$<5.0 \mathrm{E}-09$ & $\mathrm{n} / \mathrm{a}$ \\
$<4.6 \mathrm{E}-09$ & $\mathrm{n} / \mathrm{a}$ \\
$9.6 \mathrm{E}-11$ & $2.1 \mathrm{E}-10$ \\
$7.6 \mathrm{E}-11$ & $9.5 \mathrm{E}-11$ \\
$8.7 \mathrm{E}-11$ & $1.3 \mathrm{E}-10$ \\
$8.8 \mathrm{E}-11$ & $3.0 \mathrm{E}-10$ \\
$3.5 \mathrm{E}-10$ & $7.3 \mathrm{E}-10$
\end{tabular}


WHC-SD-EN-TI-308 Rev.0

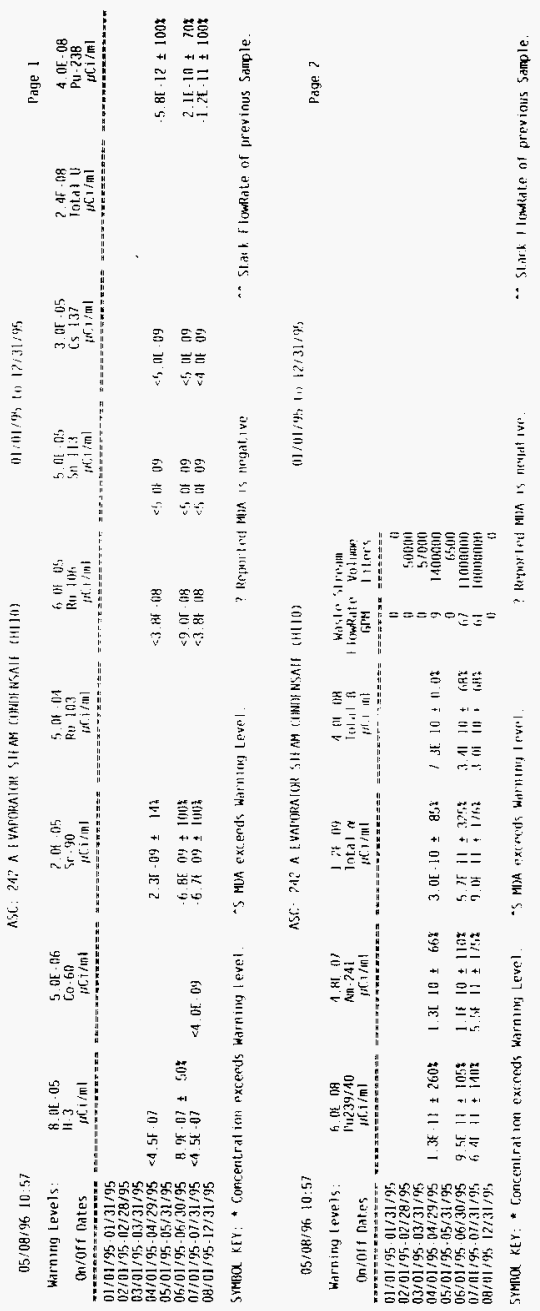




\section{WHC-SD-EN-TI-308 Rev.0}

ENUIRONMENTAL RELEASE SUMMARY REPORT

CA8: 241-A TANK FARM COOLING WATER (H115)

$01 / 01 / 95$ through $12 / 31 / 95$

\begin{tabular}{|c|c|c|c|}
\hline Radionuclide & Release, $\mathrm{Ci}$ & $\begin{array}{c}\text { Concentratj } \\
\text { Average }\end{array}$ & ons, $\begin{array}{c}\mu \mathrm{Ci} / \mathrm{ml} \\
\text { Peak }\end{array}$ \\
\hline--------- & ------- & $-\cdots---\cdot$ & ----- \\
\hline${ }^{50} \mathrm{CO}$ & 1. $7 \mathrm{E}-03$ & $2.0 \mathrm{E}-09$ & $-3 \cdot 2 E-10$ \\
\hline${ }^{90} \mathrm{sr}$ & $\mathrm{ND}$ & $-1.0 E-09$ & $8.7 \mathrm{E}-09$ \\
\hline${ }^{106} \mathrm{Ru}$ & $3.4 \mathrm{E}-02$ & $4.1 E-08$ & $2.8 E-08$ \\
\hline${ }^{113} \mathrm{Sn}$ & 8. $5 E-04$ & 1. $0 E-09$ & $-1.2 \mathrm{E}-09$ \\
\hline${ }^{125} \mathrm{Sb}$ & $\mathrm{ND}$ & $-2.6 \mathrm{E}-10$ & 1. $4 E-08$ \\
\hline${ }^{134} \mathrm{Cs}$ & $9.0 E-04$ & $1.1 E-09$ & $1.5 E-09$ \\
\hline${ }^{137} \mathrm{Cs}$ & 1. $2 E-03$ & 1. $5 E-09$ & $3.0 E-10$ \\
\hline${ }^{238} \mathrm{Pu}$ & 8. $0 E-06$ & $9.6 \mathrm{E}-12$ & $6.1 E-11$ \\
\hline $235 / 240 \mathrm{Pu}$ & $1.8 \mathrm{E}-05$ & $2 \cdot 2 \mathrm{E}-11$ & $7.6 E-11$ \\
\hline${ }^{241} \mathrm{Am}$ & $8.6 \mathrm{E}-05$ & 1. OE-10 & $2.8 E-10$ \\
\hline Total $\alpha$ & 3. $3 E-04$ & $4.0 E-10$ & $1.8 \mathrm{E}-09$ \\
\hline Total $B$ & $3.0 E-04$ & $3.6 \mathrm{E}-10$ & $6.8 E-10$ \\
\hline
\end{tabular}


08/05/96 08:44

CAB: 241 A IANK FARM COCH ING WATFR (HI]5)

01/01/95 t.o 12/31/95

\begin{tabular}{|c|c|c|c|c|c|c|c|c|c|}
\hline $\begin{array}{l}\text { Warning Levels: } \\
\text { On } 10 \text { if Dates } \\
m========x \times m x\end{array}$ & 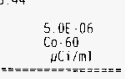 & $\begin{array}{l}2.0 F-05 \\
55-90 \\
\mu C i|m| \\
====-1 .\end{array}$ & 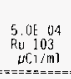 & $\begin{array}{l}6 \text { OE } 05 \\
\text { Fu- }-106 \\
\mu C i / m I \\
===-==\end{array}$ & 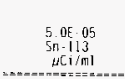 & 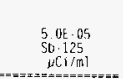 & $\begin{array}{l}2.0 \mathrm{E}-06 \\
\mathrm{Cs}-134 \\
\mu \mathrm{C} \mathrm{ci} / \mathrm{m} /\end{array}$ & $\begin{array}{l}3.00 \cdot 05 \\
6.37 \\
\mu[1 / 181\end{array}$ & 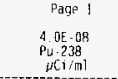 \\
\hline 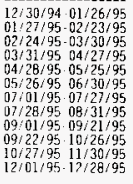 & 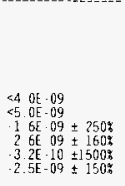 & 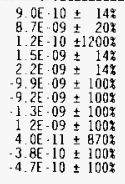 & & 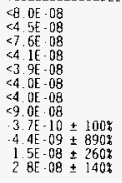 & 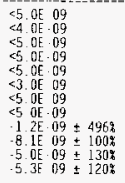 & $\begin{array}{r}1.8609 \pm 803 \% \\
136.09 \pm 900 \% \\
-26609 \pm 480 \% \\
14608 \pm 90 \%\end{array}$ & $\begin{array}{l}1.5 E .09 \pm 333 \% \\
3.3 E-10 \pm 1200 \% \\
5.12 .10 \pm 706 \\
6.4 E \cdot 10 \pm 650 \%\end{array}$ & 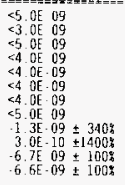 & 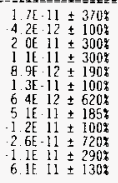 \\
\hline
\end{tabular}

$08 / 05 / 96 \quad 08-44$

$\begin{array}{cc}\text { Harning levels: } & 5.0 E 08 \\ \text { On/0ff Dates } & \text { Pu239/40 } \\ \mu C \text { Ciml }\end{array}$

SYMBCL KEY: * Concentration exceeds warning Leve

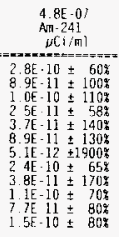

$.8 E-07$
-241
CAS: 241-A TANK FARH COOO ING WATER (HL15)

\begin{tabular}{|c|c|}
\hline $\begin{array}{l}\text { L.2E. og } \\
\text { Total } \alpha \\
\mu C \text { Cinl }\end{array}$ & $\begin{array}{c}4.0 E \cdot 08 \\
\operatorname{lotal} \beta \\
\angle C 1 / \mathrm{ml}\end{array}$ \\
\hline 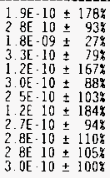 & 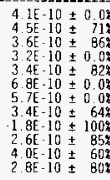 \\
\hline
\end{tabular}

^s MDA exceeds Warning Level.

Waste
Flowkat
$6 \mathrm{GP}$
-260
260
370
440
360
380
340
500
520
460
480
460
400

000000

? Reported maA is negatize
$01 / 01 / 95$ to $12 / 31 / 95$

Page 2

\begin{tabular}{|c|}
\hline 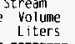 \\
\hline $\begin{array}{l}39000000 \\
5600000 \\
84000000 \\
550000000 \\
58000000 \\
66000000 \\
73000000 \\
590000000 \\
53000000 \\
920000000 \\
87000000 \\
61000000\end{array}$ \\
\hline
\end{tabular}


WHC-SD-EN-TI-308 Rev.0

ENVIRONMENTAL RELEASE SUMMARY REPORT

$08 / 05 / 96$

$08: 46$

CAR: 244-AR VAULT COOLING WATER (H116)

$01 / 01 / 95$ through $12 / 31 / 95$

Total system Flow: $4.8 E+06$ Liters $=1.3 \mathrm{E}+06$ Gallons

\begin{tabular}{|c|c|c|c|}
\hline \multirow[b]{2}{*}{ Radionuclide } & \multirow[b]{2}{*}{ Release, Ci } & \multicolumn{2}{|c|}{ Concentrations, $\mu \mathrm{Ci} / \mathrm{ml}$} \\
\hline & & Average & Peak \\
\hline - - - - - - - & $-\cdots------$ & ------- & $\sim-----$ \\
\hline${ }^{60} \mathrm{Co}$ & $2.1 E-05$ & $4.4 E-09$ & 4. $0 E-09$ \\
\hline${ }^{90} \mathrm{Sr}$ & ND & $-1.0 E-09$ & $6.5 \mathrm{E}-09$ \\
\hline${ }^{106} \mathrm{Ru}$ & $2.4 \mathrm{E}-04$ & 5. $1 E-08$ & $-3.1 E-10$ \\
\hline${ }^{113} \mathrm{Sn}$ & $1.1 E-05$ & $2.3 E-09$ & $-3.0 \mathrm{E}-09$ \\
\hline${ }^{125} \mathrm{Sb}$ & $4.0 E-05$ & 8. $3 E-09$ & $6.9 E-11$ \\
\hline${ }^{134} \mathrm{Cs}$ & $1.6 \mathrm{E}-05$ & 3. $2 \mathrm{E}-09$ & $4.0 E-09$ \\
\hline${ }^{137} \mathrm{Cs}$ & $1.5 \mathrm{E}-05$ & 3. $1 \mathrm{E}-09$ & 8. $6 \mathrm{E}-10$ \\
\hline${ }^{238} \mathrm{Pu}$ & $1.4 \mathrm{E}-07$ & 2. $9 E-11$ & 2. $8 E-10$ \\
\hline $239 / 240 \mathrm{Pu}$ & $3.7 E-07$ & $7.6 \mathrm{E}-11$ & $6.4 E-10$ \\
\hline${ }^{24} \mathrm{Am}$ & $3.3 E-07$ & $6.9 E-11$ & $1.6 \mathrm{E}-10$ \\
\hline Total $\alpha$ & $2.5 E-06$ & $5.3 E-10$ & $2.4 E-09$ \\
\hline Total B & $2.5 \mathrm{E}-06$ & $5.2 E-I O$ & 8. $3 E-10$ \\
\hline
\end{tabular}


(0)/ $45 / 96 \quad 08 / 46$

CAR 744 AR VALII T COOU ING WAIER :H]16

11/01/95 to $12 / 31 ; 95$

\begin{tabular}{|c|c|c|c|c|c|c|c|c|c|}
\hline $\begin{array}{l}\text { Warning Levels: } \\
\text { Onsoff bates } \\
========-===\end{array}$ & 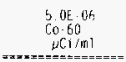 & 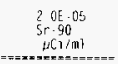 & 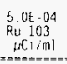 & $\begin{array}{c}6.0 E \cdot 05 \\
\text { Ru } 106 \\
\mu C i / m I\end{array}$ & 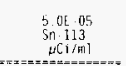 & 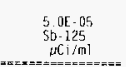 & $\begin{array}{l}2.0 \mathrm{E}-06 \\
\mathrm{Cs} \cdot 134 \\
\mu \mathrm{CH} / \mathrm{m} /\end{array}$ & 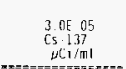 & $\begin{array}{l}4.05 \cdot 08 \\
\mathrm{Pu} \cdot 238 \\
\Delta \mathrm{C} \mathbf{1} / \mathrm{mi}\end{array}$ \\
\hline 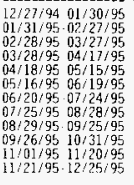 & $\begin{array}{l}5.0 E .09 \\
5.0 E 09 \\
5.0 E-09 \\
4.0 E-09 \pm 1002 \\
16 \mathrm{EO} \pm 250 \mathrm{z}\end{array}$ & 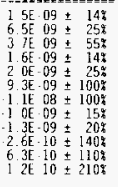 & & 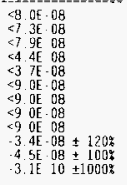 & 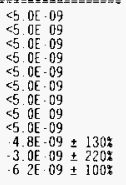 & 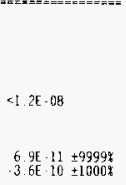 & 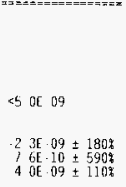 & 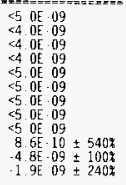 & 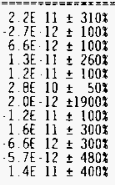 \\
\hline
\end{tabular}

08/05/36 08:46

CAR: 244 AR YAAII TOOL IHG WATER IHIIG

$01 / 01 / 95$ to $12 / 31 / 95$

Page 2

\begin{tabular}{|c|c|c|c|c|c|c|}
\hline $\begin{array}{l}\text { Warnung levels. } \\
\text { On/oft Dates }\end{array}$ & $\begin{array}{l}\text { 6. } 0 \text {. } 08 \\
P \cup 239 / 40 \\
\left.\mu \varepsilon_{5}\right] / \pi 1\end{array}$ & $\begin{array}{l}\text { A. } 8 E, 0 \mathrm{~T} \\
\mathrm{Am}-24 \mathrm{i} \\
\mu \mathrm{C} \mathbf{i} / \mathrm{ml}\end{array}$ & $\begin{array}{l}\text { I. } 2 E \cdot 09 \\
\text { Fotal } \alpha \\
\mu C \mathrm{i} / \mathrm{ml}\end{array}$ & $\begin{array}{l}\text { 4. OE } 08 \\
\text { Total } 13 \\
\alpha C i / \mathrm{m} !\end{array}$ & $\begin{array}{l}\text { Waste } \\
\text { Fomiate } \\
\text { GPM }\end{array}$ & $\begin{array}{l}\text { tream } \\
\text { Volume } \\
\text { Liters }\end{array}$ \\
\hline 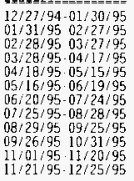 & 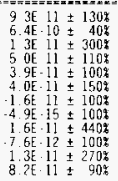 & 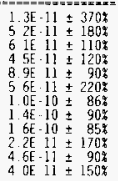 & 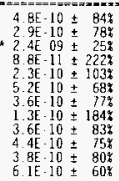 & 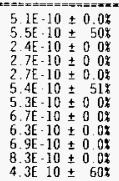 & $\begin{array}{l}2 \\
2 \\
2 \\
2 \\
2 \\
2 \\
2 \\
2 \\
2 \\
2 \\
2 \\
2 \\
2 \\
2\end{array}$ & $\begin{array}{l}460000 \\
370000 \\
378000 \\
280000 \\
370000 \\
469000 \\
460000 \\
460000 \\
370000 \\
480000 \\
260000 \\
460000\end{array}$ \\
\hline
\end{tabular}

a stack flowkate of prevrous Sampie 
WHC-SD-EN-TI-308 Rev.0

ENVIRONMENTAL RELEASE SUMMARY REPORT

$08 / 05 / 96$

$08: 46$

CBC: B-PLANT COOLING WATER (H117)

$01 / 01 / 95$ through $12 / 31 / 95$

Total System Flow: $3.0 \mathrm{E}+09$ Liters $=7.9 \mathrm{E}+08$ Gallons

Radionuclide

${ }^{60} \mathrm{Co}$

${ }^{90} \mathrm{Sr}$

${ }^{106} \mathrm{Ru}$

${ }^{113} \mathrm{Sn}$

$125 \mathrm{Sb}$

${ }^{134} \mathrm{Cs}$

$137 \mathrm{Cs}$

Total $\alpha$

Total B
Concentrations, $\mu \mathrm{Ci} / \mathrm{ml}$

Release, Ci

7. $4 \mathrm{E}-03$

1. $1 E-03$

1. $6 \mathrm{E}-01$

9. $3 E-03$

3. $1 E-03$

ND

5. $1 \mathrm{E}-03$

1. $1 \mathbf{E}-03$

3. $8 \mathrm{E}-03$

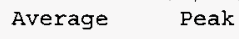

1.7E-09 5.1E-09

3. $6 \mathrm{E}-10 \quad 7.9 \mathrm{E}-10$

1. $3 E-09 \quad 2.9 E-09$ 


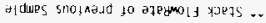

2 abed

$96 / 18 / 210796 / 10 / 10$

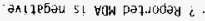

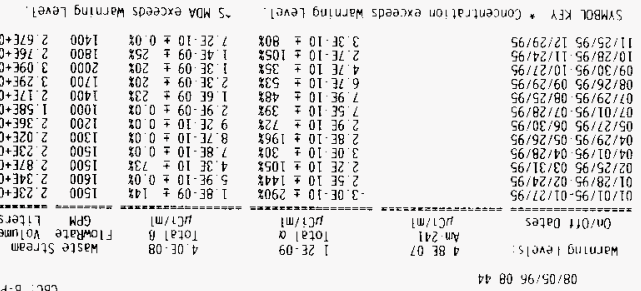

Joules 5noinasd to ageymotd YOPTS ..

an!legas 5 ! Yan pazhoday $i$

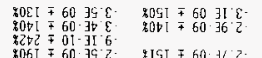

65 $50.70 \cdot 35$

60.30 .5
$60-305$

$60 \cdot 30.5$
60.30 .5
60.70 .9

$60.70 \mathrm{9}$
$60.30 \mathrm{c}$
$60.30 \mathrm{p}$

$151=60 \cdot \mathrm{HC}$

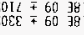

t6.09 760.30

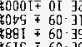

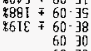

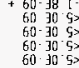

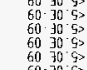

60.30 .5
6070.9
$60.70 \%$

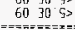

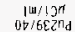

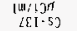

50.30

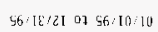

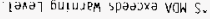

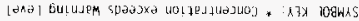

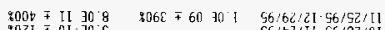

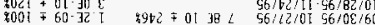

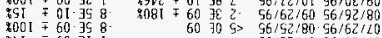

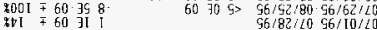
$x_{k+1}+600$

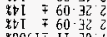

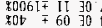
$56 / 92 / 50 \quad 96 / 62 / 70$
$56 / 82 / 00.56 / 10 / 70$

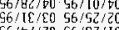
$56 / 25 / 2096 / 82 / 10$ รeา? $110 / 10$

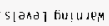
wi $8046 / 40 / 30$ 
WHC ENVIRONMENTAL RELEASE SUMMARY

CSL: PUREX CHEMICAL SEWER (H118)

$01 / 01 / 95$ through $12 / 31 / 95$

Total system Flow: $1.8 E+08$ Liters $=4.9 E+07$ Gallons

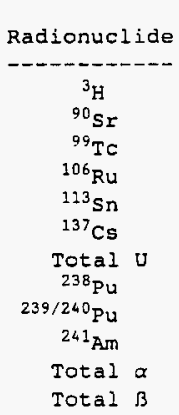

Release, Ci
ND
$6.3 E-04$
$2.3 E-04$
ND
ND
ND
$5.1 E-05$
$2.6 E-05$
$5.1 E-04$
$9.1 E-05$
$3.2 E-05$
$1.1 E-04$

$\begin{array}{cc}\text { Concentrations, } \mu \mathrm{Ci} / \mathrm{ml} \\ \text { Average } & \text { Peak } \\ -0--- & ----- \\ <4.5 \mathrm{E}-07 & \mathrm{n} / \mathrm{a} \\ 3.4 \mathrm{E}-09 & 6.7 \mathrm{E}-09 \\ 1.2 \mathrm{E}-09 & 2.4 \mathrm{E}-09 \\ <6.2 \mathrm{E}-08 & \mathrm{n} / \mathrm{a} \\ <5.0 \mathrm{E}-09 & \mathrm{n} / \mathrm{a} \\ <4.4 \mathrm{E}-09 & \mathrm{n} / \mathrm{a} \\ 2.7 \mathrm{E}-10 & 5.9 \mathrm{E}-10 \\ 1.4 \mathrm{E}-10 & 8.4 \mathrm{E}-10 \\ 2.8 \mathrm{E}-09 & 1.3 \mathrm{E}-08 \\ 4.9 \mathrm{E}-10 & 2.0 \mathrm{E}-09 \\ 1.7 \mathrm{E}-10 & 4.0 \mathrm{E}-10 \\ 5.9 \mathrm{E}-10 & 8.8 \mathrm{E}-10\end{array}$




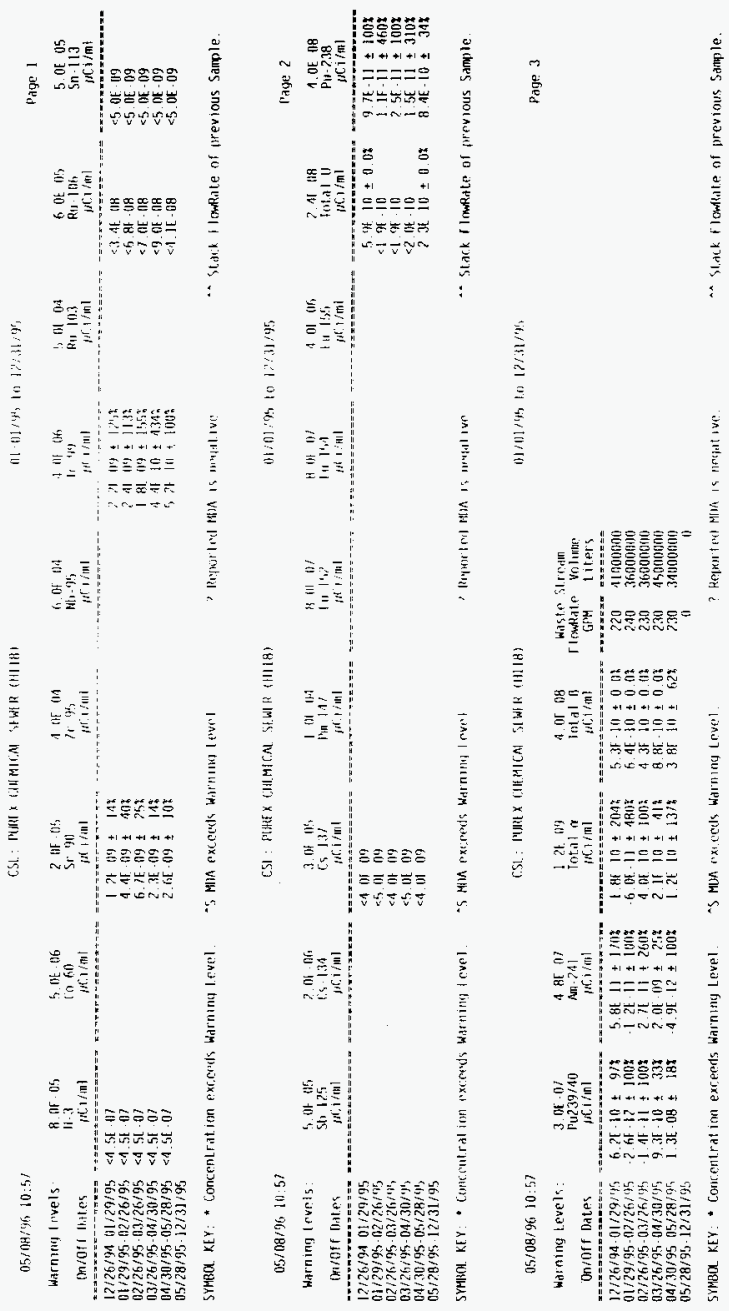


WHC-SD-EN-TI-308 Rev.0

This page intentionally left blank. 
WHC-SD-EN-TI-308 Rev.0

3.4200 West Area 
WHC-SD-EN-TI-308 Rev.0

WHC ENVIRONMENTAL RELEASE SUMMARY

$03 / 23 / 96$

$09: 56$

207-SL: 222-2 LABORATORY CHEMICAL SEWER (H101)

$01 / 01 / 95$ through $12 / 31 / 95$

Total system Flow: $2.4 \mathrm{E}+06$ Liters $=6.2 \mathrm{E}+05$ Gallons

Radionuclide

${ }^{0}{ }_{\mathrm{Sr}}$
-----0

${ }^{106} \mathrm{Ru}$

${ }^{113} \mathrm{Sn}$

$137 \mathrm{Cs}$

${ }^{238} \mathrm{Pu}$

$239 / 240 \mathrm{Pu}$

${ }^{241} \mathrm{Am}$

Total $\alpha$

Total $\beta$
Release, Ci

----------

9. 9 $\mathrm{E}-06$

ND

ND

1. 2E-05

ND

3. $0 \mathrm{E}-07$

4. $7 \mathrm{E}-07$

6. 6E-07

3. 1 E-06

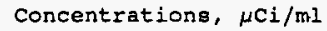

4. 2E-09

$<8.2 \mathrm{E}-08$

$<5.2 \mathrm{E}-09$

5. 3E-09

$-5.6 E-12$

1. $3 E-10$

2. $0 \mathrm{E}-10$

2. $8 \mathrm{E}-10$

1. $3 E-09$

$n / a$

$n / a$

9. $9 \mathrm{E}-09$

2. $4 \mathrm{E}-11$

2. $9 E-10$

3. $4 E-10$

3. $9 E-10$

6. $8 E-09$ 
WHC-SD-EN-TI-308 Rev.0

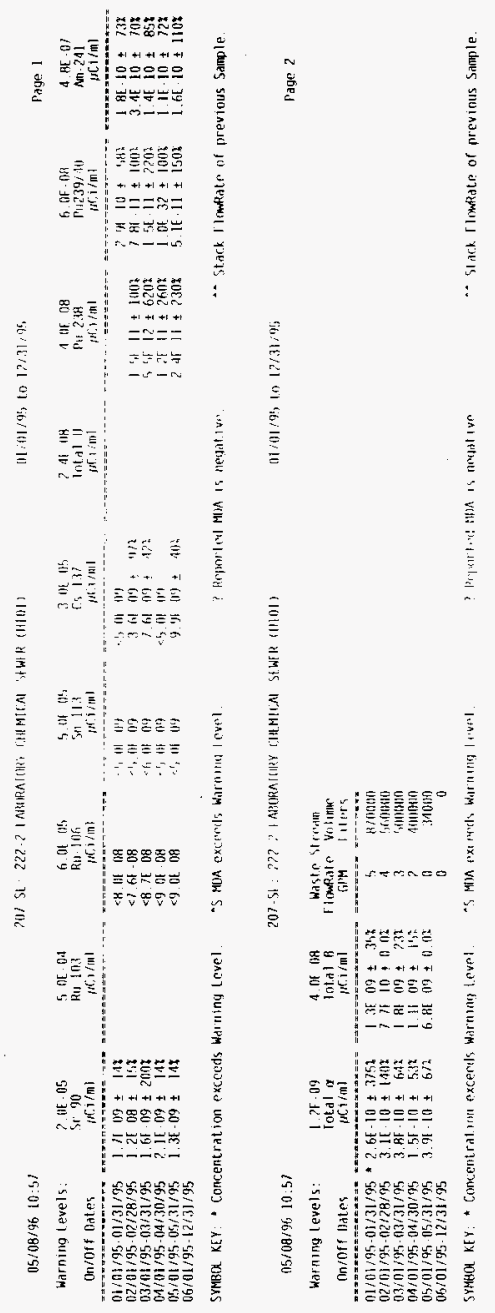


WHC-SD-EN-TI-308 Rev.0

ENVIRONMENTAL RELEASE SUMMARY REPORT

$08 / 05 / 96$

$08: 46$

2904-ZA: 234-5-Z PLANT COOLING WATER (H103)

$01 / 01 / 95$ through $12 / 31 / 95$

Total System Flow: $7.2 E+06$ Liters $=1.9 E+06$ Gallons

Radionuclide
${ }^{137} \mathrm{Cs}$
${ }^{238} \mathrm{Pu}$
$239 / 240 \mathrm{Pu}$
${ }^{241} \mathrm{Am}$
Total $\alpha$
Total $B$

Release, Ci
ND
$7.6 \mathrm{E}-06$
$1.1 \mathrm{E}-04$
$3.6 \mathrm{E}-05$
$1.4 \mathrm{E}-04$
$9.6 \mathrm{E}-06$

Concentrations, $\mu \mathrm{Ci} / \mathrm{ml}$

Average Peak

-.-.-.-. -.-.-.

$<4.2 \mathrm{E}-09 \mathrm{n} / \mathrm{a}$

1. $1 \mathrm{E}-09 \quad 2.6 \mathrm{E}-09$

1. $5 \mathrm{E}-08 \quad 3.1 \mathrm{E}-08$

5. $0 \mathrm{E}-09 \quad 9.6 \mathrm{E}-09$

1. $9 E-08 \quad 4.1 E-08$

1. $3 E-09 \quad 2.2 E-09$ 


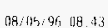

2904-2A 234 与 Z P1 AN CHOH. ING WATER (HLO3)

\begin{tabular}{|c|c|c|c|c|c|c|c|c|c|c|}
\hline $\begin{array}{l}\text { Warmina l evels. } \\
\text { Oniofi Udates } \\
=========-=\end{array}$ & $\begin{array}{l}2.0[-05 \\
\sin 90 \\
\mu c i / m 1\end{array}$ & 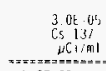 & 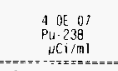 & 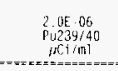 & 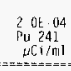 & 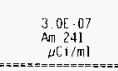 & 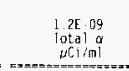 & 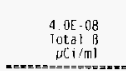 & $\begin{array}{c}\text { Waste } 5 \\
\text { flowfate } \\
\text { GPM } \\
\end{array}$ & 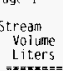 \\
\hline 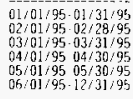 & & $\begin{array}{l}= \\
<3.0 \mathrm{E} \quad 09 \\
<4.0 \mathrm{E} \quad 09 \\
<4.0 \mathrm{E} \quad 09 \\
<3.0 \mathrm{E} .09 \\
<5.0 \mathrm{E}-09\end{array}$ & 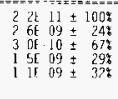 & 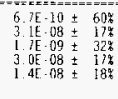 & & 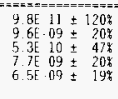 & 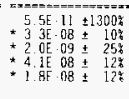 & 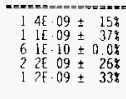 & $=x====$ & $\begin{array}{r}1100000 \\
990000 \\
1500000 \\
1700000 \\
1900000 \\
0\end{array}$ \\
\hline
\end{tabular}

SYMFOL KEY: " Concentration exceeds warning Level

? Reported MEA is negdtive

- stack homfate of previous 5ample. 
WHC-SD-EN-TI-308 Rev.0

This page intentionally left blank. 


\subsection{DEFINITION FILES USED}

This section contains definition files used to generate release and trend reports from the ERS database. Only the portions from each definition file, used to generate reports, are included in this document. For Example, the GROUPS.DEF file contains many facility definitions that are not used for the annual effluent reports, only the portions used for the annual effluent reports are included in this document.

The ERS definition files included are: EIS_ODIS.DEF, DOSE_MOD.DEF, GROUPS.DEF, and PLANTS.DAT. 


\section{WHC-SD-EN-TI-308 Rev.0}

\section{EIS ODIS.DEF}

Sheet 1 of 3

2. $185 E-0581$ R DKR003002A950101951231 16105 -KE STORAGE BASIN ROOF VENTS (Y245-Y248) $Y 245 \times 245 \quad Y 246 \times 246 \quad 4247 \times 247 \times 248 \times 248$

2.061E-06 6 I R DKR003004A950101951231 16 105-KW STORAGE BASIN ROOF VENTS (Y234-Y236) Y234 X234 Y235 X235 Y236 ×236

$1.525 E-0721$ R DKRO03013A950101951231 16 1705-KER - 27 FT LAB EXHAUST (Y244) $Y 244 \times 244$

7. 420E-07 2 I R DKRO03001A950101951231 16 1706-KE LAB EXHAUST (Y243)

$Y 243 \times 243$

6.595E-06 21 R ONRD01002A950101951231 15 115-N STACK (Y211) Y211 X211

$3.916 E-072$ I R DNROD1011A950101951231 15 105-N 14 FT STACK (Y259)

$4.535 E-0721 R$ ONRO01012A950101951231 $15107-N$ STACK (Y265)

Y 265 Y 266

$0.000 E+0031 R$ DNR001006A950101951231 $15109-N-Z-1$. 109-N BLDG. ZONE I STACK (Y221) Y221 Y222 Y223

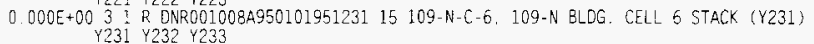

$7.135 E-074$ I R AEP151151A950101951231 $1291-A-1$. PUREX MAIN STACK (A552: A552 A566 $\times 552$ A511

1.291E-072 1 R AEP151152A950101951231 1296 -A-1. FILTR N \& O CELLS. PR ROOM (A540)

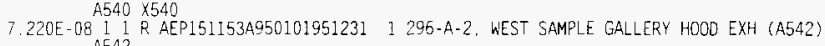
$\mathrm{A} 542$

9. 627 E-0B 1 I R. AEP151154A950101951231 1 296-A-3. PUREX EAST SAMPLE GAL HOOD EXHAUST (A543)

$6.126 E-072$ R AEP151157A950101951231 I 296-A-5A ANO 296-A-5B. 202-A LAB HOOD EXHAUST (A545. A546)

$3.938 E-07 \stackrel{A 546}{1}$ A545 AEP151158A950101951231 i 296-A.6. EAST SAMPLE GALLERY \& U-CELL (A547)

$5.908 E-07 \frac{1}{4} \frac{1}{8}$ R AEP151159A950101951231 1 296-A-7. WEST SAMPLE GALLERY \& R-CELL (A548)

$4.595 E-07$ A ? R AEF151160A950101951231 1 296-A-8. WEST P \& 0 GALLERIES (A549)

$2.407 E-07 \frac{1}{A 550}$ R AEP151162A950101951231 $1296-A-10$. PUREX EOUIPMENT DISPOSAL TUNWEL 2 (A550)

1.553E-07 1 1 R AEP151156A950101951231 1 296-A-14, 293-A 'OUTBACK FACILITY' EXHAUST (A544)

$2.759 E-072$ R AEB151176A950101951231 2 291-B-1. B-PLANT MAIN STACK (B691)

B69] X691
$4.814 E-081$ R AEB151181A950101951231 2 296-B-5, 221-BB BUILDING EXHAUST (B686)

6.546E-07 2686 R AEB151185A950101951231 2 296-B-10, 225 BUILOING EXHAUST (8748)

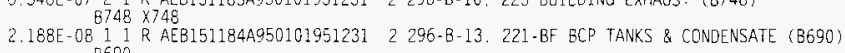

C.000E+002: R AEF151164A950101951231 3 296-A-12, 244-AR VAIULT: VESSEL VENT (E058)

$\begin{array}{rl}0.000 E+00 & 11 \\ 0.058 & \text { REF151165A950101951231 3 296-A-13, 244-AR VAULT: CANYON \& CELL (E052) }\end{array}$

2.013E-07 5 C R AEF $151169 A 950101951231$ E059 E026 X059 E039 E040 X039

$0.000 E+001$ 1 R AEF151170A950101951231 3296-A-18. 241-101-AY ANNULUS (E060)

$0.000 E+00 I 1$ R AEF151171A950101951231 3 296-A-19. 241-102-AY ANNULUS (E061)

3.501E-08 I I R AEF151172A95010:95123: 3 296-A-20. 241-AX T F. 101. 102-AZ ANNULI (E197)

7 439E-07 2 R AEF151173A950101951231 3296-A-21, 242-A EVAP/CRYSTALIZER BLDG. (E645)

2.100E-08 3 I R AEF151174A950101951231 3 296-A-22. 242-A EVAP/CRYSTALIZER \& VESSEL VENTILATION (E643)

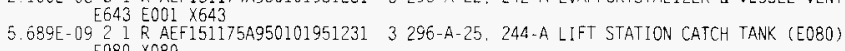

$6.345 E-08] 1$ R AEF $151559 A 9501019512313296-4-26.204$-AR WASTE BLDG. EXHAUST (E297)

$3.938 E-083$ : $\mathrm{E} 297$ AEF 251545 A950101951231 $3296-A-27$. ALL AN TANK EXHAUSTS (E270)

E270 E933 X270

$2.013 E-07$ I I R AEF151546A950101951231 3296-A-28. ALL AN ANAULL] (E272)

$3.063 E-0821$ R AEF151555A950101951231 3 296-A-29. ALL AN TANK EXHAUSTS (E901) E901 $\times 901$ 


\section{EIS ODIS.DEF (cont.)}

Sheet 2 of 3

2.013E-07 I 1 R AEF151556A950101951231 3 296-A-30. ALL AN TANK ANNULI EXHAUSTS (E903)

$3.063 E-0831$ R AEF151579A950101951231 3 296-A-40. ALL AP TANK EXHAUSTS (EO13)

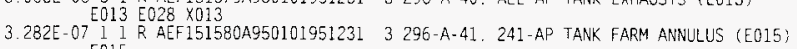
E015

9.189E-09?2 R AEF151569A950101951231 3 296-B-28, 244-BX SALT WELL TANK \& ANN (EB86)

$1.641 E-0721$ R AEF151191A950101951231 3 296-C-5. 244-CR VAULT CELL \& VESSEL VENT (ED69) E069 X069

$0.000 E+0031$ R AEF $151600 A 9501019512313296-6-6$. TANK C-106 EXHAUSTER (ED83)

E083 E084 X083

$0.000 E-0011$ R AEF 151606 A950101951231 3296-C-7. TANK $C-103$ VAPOR MIXER EXHAUSTER (E044)

$1.313 E-072$ EOA $\mathrm{R}$ AEF151200A950101951231 $3296-\mathrm{P}-16, \mathrm{C}-105$ \& C.106 TANK EXHAUST (E068)

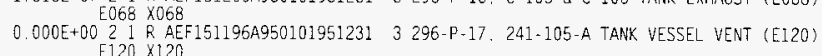

7 .220E-08 1 I R AEF 151028A950101951231 3 296-P-31, 209-E CRITICALITY LABORATORY EXHAUST (E209)

2.626E-098 I R AEF1516014950101951231 3 296-P-32, 33. 34 ROTARY MODE CORE SAMPLERS (E301-E399)

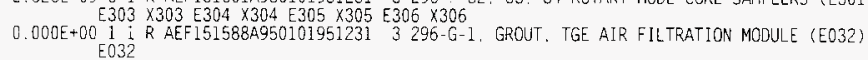

$2.626 E-0711$ R AET151036A950101951231 $27296-E-1$, EFFLUENT TREATMENT FACILITY (E036)

4. $157 E-091$ I R. AWL216232A950101951231 5 296-5-16. 219-S VALLT AND WASTE TANKS EXHAUSTS (S264)

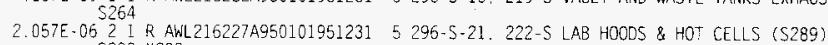

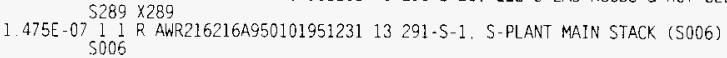

4.157E-OB I I R AWR216218A950101951231 13 296-5-2. 202-S NORTH SAMPL. GAL. HOOD. \& PR ROOM (5032)

$9.627 E-09$
5008

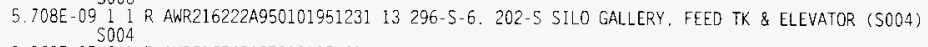

3.063E-072 ] R AWR216548A950101951231 13 295-S-7W AND 296-S-7E, 233-S G.H. \& PROCESS V.V. (S015. 5016)

$\left.\begin{array}{rl}5016 & 5015 \\ 1.750 E-07] & 1\end{array}\right]$ R AWU216256A950101951231 $14291 \cdot U-1$, U-PLANT MAIN STACK (U771)

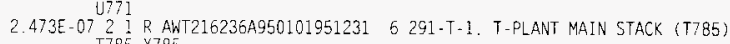

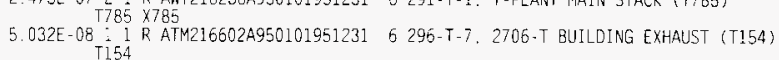

$1.225 E-0611$ R AWT216249A95010195:23i 6 296-T-13. T-PLANT VENTILATION AIR (T786)

I. 269E-08 11 R AWZ216547A950101951231 29 296-T-11. 224-T BLDG W FAN EXH. (T783)

3. 063E-07 T7 T R AWZ216248A950101951231 29 295-T-12, 224-T BLDG. E FAN EXH. (T784)

$3.938 E-08$ i 1 R AWF216215A950101951231 8 296-P-22. ALL SY TANK ANNULI (W191)

3.720E-08 4 \& ANF216508A95010195123? 8 296-P-23 AND 296-P-28. ALL SY TANKS (W190, W195)

W190 X190 W195 X195
$1.904 E-07 ? 1$ R AWF216231A950101951231 8 296-S-15. ALI SX TANK5 (W111:

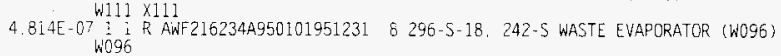

3.720E-09 21 R AWF216560A950101951231 8 296-S-22, 244-S SAL TWELL RECEIVER \& ANNUL. (W880)

6.554E-08 11 R AWF216253A950101951231 \& 296-T-17, 242-T WASTE EVAP. ANO CELL EXH. (W117)

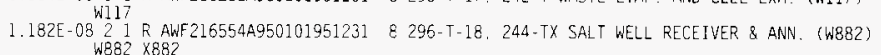

$0.000 E+0022$ R AWF2165034950101951231 $8295-U-11$. 244-U SALT WELL EXHAUST (W884)

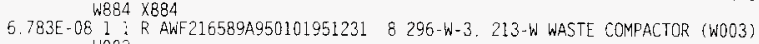

1 G19E-06 $1 \frac{1}{1}$ R AWS2160104950101951231 26 696-W-1, WSCF MAIN STACK (W010) 


\section{WHC-SD-EN-TI-308 Rev.0}

\section{EIS_ODIS.DEF (cont.)}

Sheet 3 of 3

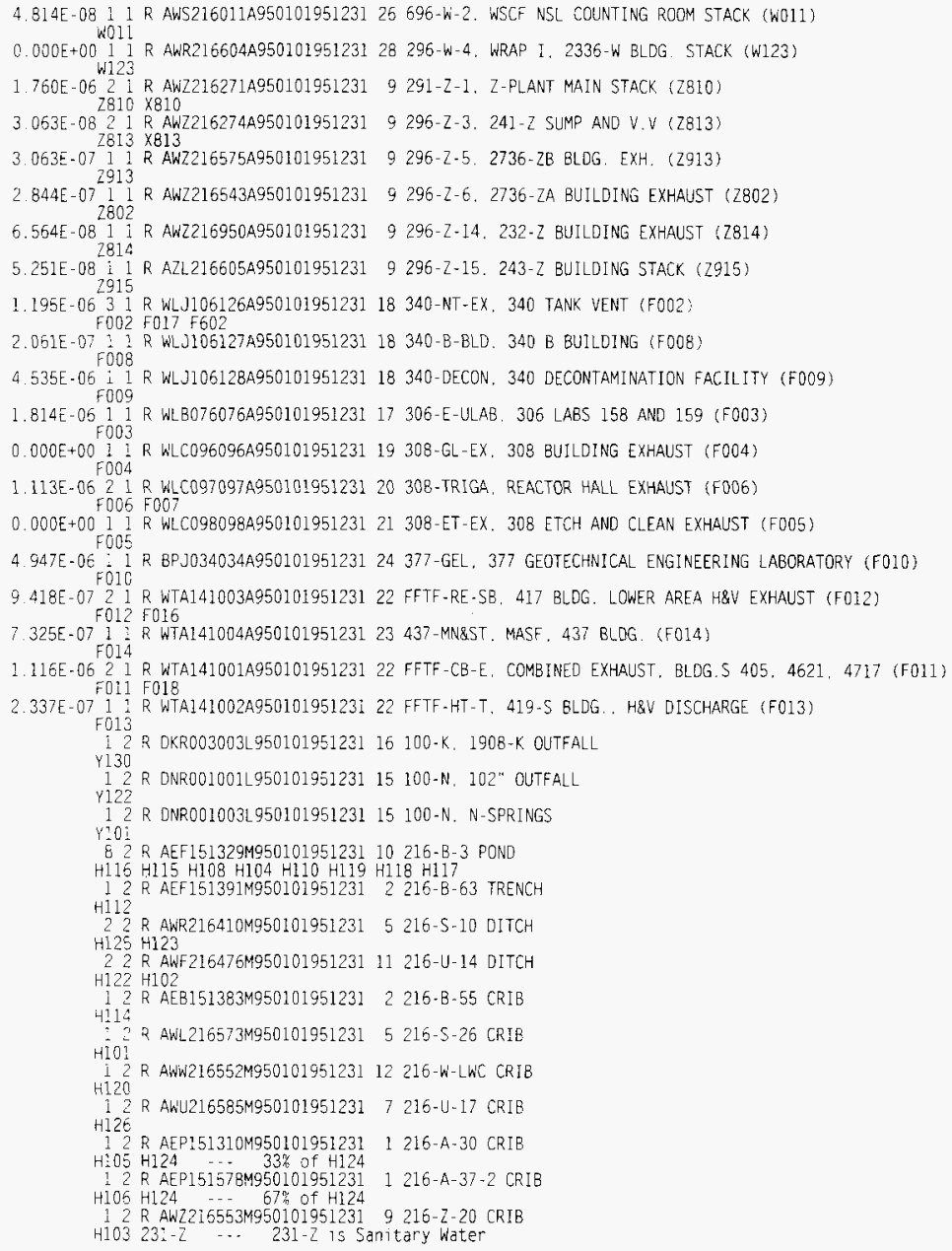




\section{WHC-SD-EN-TI-308 Rev.0}

\section{DOSE MOD.DEF}

100_AIR 282 Radionucildes Discharged to the Atmosphere from 100 Areas

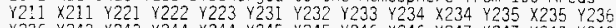
$\times 236 Y 243 \times 243$ Y $244 \times 244 \quad Y 245 \times 245 Y 246 \times 245 \times 247 \times 247 \quad Y 248 \times 248$ Y 259 Y 265 Y 265

$200 E$ AIR 842 Radionuclides Discharged to the Atmosohere from 200-East Area A540 X540 A542 X542 A543 A544 A545 X545 A546 X546 A547 X547 A548 $\times 548$ A549 X549 A550 A552 A511 X552 A940 A566 A578 B685 B690 B691

$\times 691 \quad B 698 \quad 8748 \times 748 \quad E 013 \quad E 028 \times 013 \quad E 015 \quad E 032 \quad E 036 \quad E 039 \quad E 040 \times 039$

E044 E052 E058 X058 E059 E026 X059 E060 E061 E068 X068 E069 X069

E080 X080 E083 E084 X083 E120 X120 E197 E209 E270 E933 X270 E272

$E 297 \quad E 303 \times 303 E 304 \times 304 \quad E 305 \times 305 E 306 \times 306 \quad E 643$ E001 $\times 643 \quad E 645$ $\times 645$ E886 X886 E901 X901 E903

200W_AIR 482 Radionuclides Discharged to the Atmosphere from 200-West Area $5004500650085015501650325264 \quad 5289 \times 289$ T154 T783 T784 T785

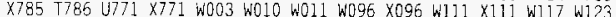
W145 X145 W190 X190 \$191 W195 X195 W880 X880 W882 X882 W884 X884 $2802 Z 810 \times 810 Z 813 \times 813 Z 814 \quad 2913 \times 913 Z 915$

$300 \mathrm{AlR}$ 12 2 Radionuclides Discharged to the Atmosphere from the $300 \mathrm{Area}$ F001 F002 F017 F602 F003 F004 F005 F006 F007 F008 F009 F010

400_AIR 9 ? Radionuclides Discharged to the Atmosphere from the 400 Area F011 F018 F024 F012 F016 F013 F014 F019 F024

100_WATER 32 Radionuclides Discharged to the Columbia River from 100 Areas $Y 101$ Y122 Y130

200_WATER18 2 RadionuC7 ides Discharged to Ground from 200 Areas

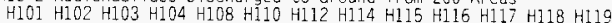
H120 H122 H123 H124 H126

300 WATER ? 2 Radionuciides Discharged to Ground from 300 Areas F021 
WHC-SD-EN-TI-308 Rev.0

\section{GROUPS.DEF}

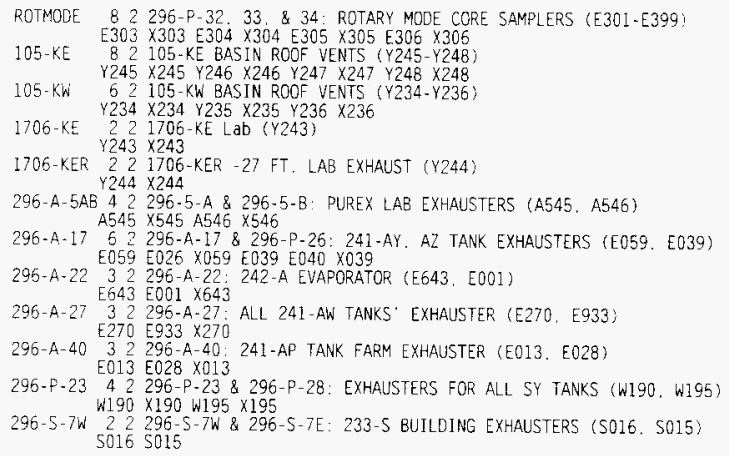




\section{WHC-SD-EN-TI-308 Rev.0}

\subsection{ERS FILE SETUPS}

The ERS files setup data is contained in the audit reports for the airborne and liquid effluent EDP codes. This section contains the audit files for the radioactive airborne and liquid effluent streams. 
WHC-SD-EN-TI-308 Rev.0

This page intentionally left blank. 
WHC-SD-EN-TI-308 Rev.0

5.1 Audit Report for Air Effluent Files 


$$
05 / 13 / 96
$$

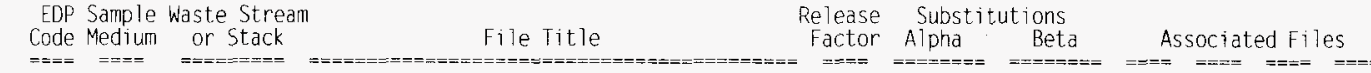

\begin{tabular}{|c|c|c|c|}
\hline $\begin{array}{l}\text { 1. } A 511 \\
\text { 2. A539 } \\
\text { 3. } A 540 \\
\text { 4. } A 542 \\
\text { 5. } A 543\end{array}$ & $\begin{array}{l}1 / 2 \\
1 / 1 \\
1 / 1 \\
1 / 1 \\
1 / 1\end{array}$ & $\begin{array}{l}291-A-1 \\
296-A-24 \\
296-A-1 \\
296-A-2 \\
296-A-3\end{array}$ & $\begin{array}{l}\text { PUREX MAIN STACK } \\
\text { PUREX AMMONIA SCRIBBBER } \\
\text { PUREX L. N Q CELIS \& PR ROOM } \\
\text { PUREX WEST SAMPLE GAL. HOOD EXHAUST } \\
\text { PUREX EAST SAMPLE GAL. HOOD EXHAUST }\end{array}$ \\
\hline $\begin{array}{l}\text { A54 } \\
\text { A54 } \\
\text { A54 } \\
\text { A54 } \\
\text { A54 }\end{array}$ & $\begin{array}{l}1 / 1 \\
1 / 1 \\
1 / 1 \\
1 / 1 \\
1 / 1\end{array}$ & $\begin{array}{l}296-A-14 \\
296-A-5 A \\
296-A-5 B \\
296-A-6 \\
296-A-7\end{array}$ & $\begin{array}{l}\text { 293-A 'OUTBACK FACILITY' EXHAUST } \\
\text { PUREX LAB: W WEST EXHAUSTIER } \\
\text { PUREX LAB: W EAST EXHAUSTER } \\
\text { PUREX EAST SAMPLE GAL. \& U CELL } \\
\text { PUREX WEST SAMPLE GAL. \& U CELL }\end{array}$ \\
\hline
\end{tabular}

\begin{tabular}{|c|c|c|c|c|c|c|}
\hline $\begin{array}{l}0.87 \\
0.91 \\
0.73 \\
0.73 \\
0.73\end{array}$ & $\begin{array}{l}\text { Pu239/40 } \\
\text { Pu239/40 } \\
\text { Pu239/40 }\end{array}$ & $\begin{array}{l}5 r-90 \\
5 r-90 \\
5 r-90 \\
S r-90\end{array}$ & $\begin{array}{r}4552 \\
\times 539 \\
\times 540 \\
\times 542\end{array}$ & $\times 552$ & A940 & A566 \\
\hline $\begin{array}{l}0.73 \\
0.73 \\
0.73 \\
0.73 \\
0.73\end{array}$ & $\begin{array}{l}\text { Pu239/40 } \\
\text { Pu239/40 } \\
\text { Pu239/40 } \\
\text { Pu239/40 } \\
\text { Pu239/40 }\end{array}$ & $\begin{array}{l}5 r-90 \\
5 r-90 \\
5 r-90 \\
5 r-90 \\
5 r-90\end{array}$ & $\begin{array}{r}\times 545 \\
\times 546 \\
\times 547 \\
\times 548\end{array}$ & & & \\
\hline $\begin{array}{l}0.73 \\
0.73\end{array}$ & $\begin{array}{l}\text { Pu239/40 } \\
\text { Pu239/40 }\end{array}$ & $\begin{array}{l}\mathrm{Sr}-90 \\
\mathrm{Sr}-90\end{array}$ & $\times 549$ & & & \\
\hline $\begin{array}{l}0.52 \\
0.73 \\
0.91\end{array}$ & Pu239/40 & $5 r-90$ & $\begin{array}{l}\text { A511 } \\
\text { A552 }\end{array}$ & $\begin{array}{l}\times 552 \\
A 511\end{array}$ & $\begin{array}{l}4940 \\
\times 552\end{array}$ & $\begin{array}{l}\text { A566 } \\
\text { A940 }\end{array}$ \\
\hline $\begin{array}{l}0.91 \\
0.91 \\
0.91 \\
0.91 \\
0.91\end{array}$ & $\begin{array}{l}\text { Pu239/40 } \\
\text { Pu239/40 } \\
\text { Pu239/40 } \\
\text { Pu239/40 } \\
\text { Pu239/40 }\end{array}$ & $\begin{array}{l}S r-90 \\
S r-90 \\
S r-90 \\
S r-90 \\
S r-90\end{array}$ & & & & \\
\hline $\begin{array}{l}0.91 \\
0.73 \\
0.73 \\
0.73\end{array}$ & $\begin{array}{l}\text { Pu239/40 } \\
\text { Pu239/40 }\end{array}$ & $\begin{array}{l}S r-90 \\
S r-90\end{array}$ & A552 & A511 & $\times 552$ & A566 \\
\hline
\end{tabular}

0.73 Pu239/40 Sr-90
PUREX WALL EXHAUSTER F $-3-9$

PUREX MAIN STACK

P21-BB BUILDING EXHAUST

221-BF BCP TANKS \& CONDENSATE

221 -B BLDG \& CANYON (B-PLANT MAIN STK)

291-B-1

221-B VESSEL VENT. SYSTEM \#2

Release Substitutions $==-\bar{m}== \pm=\quad== \pm=\quad==$ 
AUDI $\Gamma$

$$
05 / 13 / 96
$$
EDP Sample waste Stream
Code Medium or Stack

$\begin{array}{lll}\text { 26. } & \mathrm{B} 748 & 1 / 1 \\ \text { 27. } & \mathrm{E} 001 & 1 / 2 \\ \text { 28. } & \mathrm{E} 013 & 1 / 1 \\ \text { 29. } & \mathrm{E} 015 & 1 / 1 \\ \text { 30. } \mathrm{F} 026 & 1 / 2 \\ \text { 31. } & \mathrm{E} 028 & 1 / 2 \\ \text { 32. } & \mathrm{E} 032 & 1 / 1 \\ \text { 33. } & \mathrm{E} 036 & 1 / 1 \\ \text { 34. } & \mathrm{E} 039 & 1 / 1 \\ \text { 35. } & \mathrm{E} 040 * & 1 / 2\end{array}$

296-B-10 296-A-22 $296-A-40$ 296-A-41 296-A-17

296-A-40 296-G- 1 $296-\mathrm{F}-1$ $296-\mathrm{P}-26$ $296-P-26$
36. $\mathrm{E} 0521 / 1$

37. $\mathrm{E058} \quad 1 / 1$

38. E059 1/1

39. $E 060 \quad 1 / 1$

40. E061 1/1

41. $\mathrm{E} 068 \mathrm{H} 1 / 1$

42. E069 1/1

43. $E 080 \quad 1 / 1$

44. $E 083 * 1 / 1$

45. $E 084^{\star} 1 / 2$

46. E120 $1 / 1$

47. E197 1/1

48. E209 1/1

49. E270 1/1

50. $\mathrm{E} 2721 / 1$

\section{6-A-13}

296-A-12 296-A-17 296-A-18 296-A-19

$296-P-16$ $296-C-5$ $296-\mathrm{A}-25$ $296-\mathrm{C}-6$ $296-\mathrm{C}-6$

\section{$296-P-17$} $296-A-20$ 296-P-31 296-A-27 $296-A-28$
File Title

Rejease Factor

Alpha

Beta

Associated Files

225-B BUILDING \& CELLS

242-A EVAPORATOR

241-AP TANK FARM EXHAUST

241-AP TANK FARM ANNULUS

241-AY, AZ TANKS

241-AP TANK FARM EXHAUST

TGE AIR FILTRATION MODULE (243-6-8)

TE ATR FIL

241-AY. AZ EXHAUUSTER BACKUP RP 296-A-17 241-AY. AZ EXHAUSTER, BACKUP TO 296-A-17

0.73

0.87

$0.80 \quad$ Pu239/40

0.80 Pu239/40 Sr-90

0.87

0.87

0.73

0.72

0.70

0.70

Pu239/40 Sr-90

Pu239/40 Sr-90

244-AR VAULT: CANYON \& CELL

244 -AR VALL T. VESSEL VENT

$241-A Y$. AZ TANKS

101-AY ANNULUS

0.73 Pu239/40 Sr-90

0.73 Pu239/40 Sr-90

0.71 Pu239/40

0.73 Pu239/40 Sr-90

102-AY ANNULUS

0.73 Pu239/40 $5 r-90$

105-C \& 106-C TANK EXHAUSTER

244-CR VAULLT CELL \& VESSEL VENT

244-A LIFT STATION CATCH TANK

296-C-6 STACK SAMPLER

0.73

0.83

0.81 Pu239/40 Sr-90

0.73

0.73

$0.73 \mathrm{Pu} 239 / 40 \mathrm{Sr}-90$

0.84 Pu239/40 Sr-90

$0.91 \mathrm{Pu} 239 / 40 \mathrm{Sr}-90$

0.52

$0.47 \mathrm{Pu} 239 / 40 \mathrm{Sr}-90$

$\times 748$

$E 643 \times 643$

E028 $\times 013$

E059 $\times 059$

E013 $\times 013$

$\times 039$

E039 $\times 039$

E026 X059

$\times 068$

$\times 069$

$\times 080$

E084 $\times 083$

E083 $\times 083$

ALL AZ ANNUL I

209-E CRITICAL ITY LABORATORY EXHAUSS

ALL AW TANKS 


$$
\text { 05/13/96 }
$$

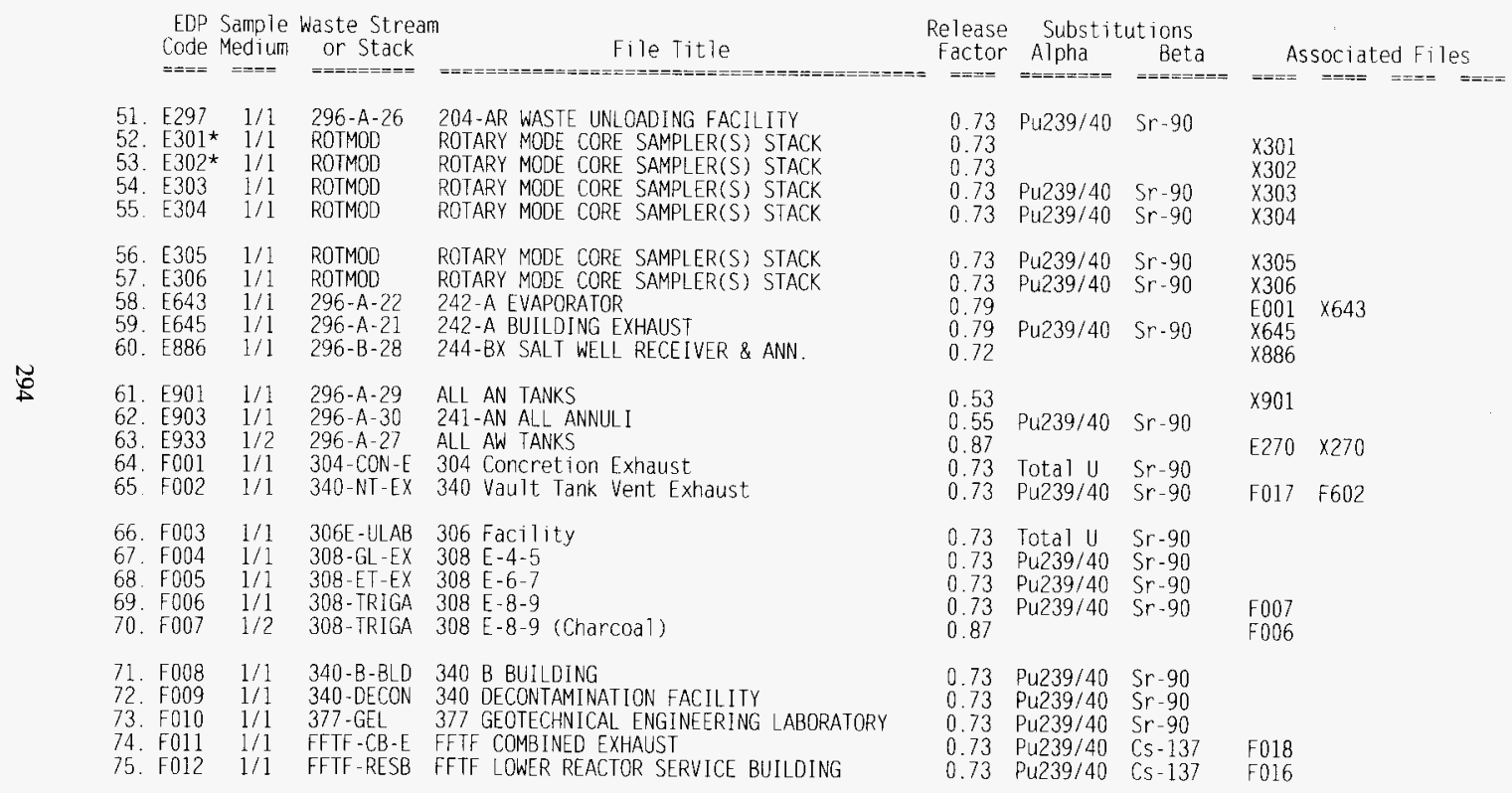




$$
\text { 05/13/96 }
$$

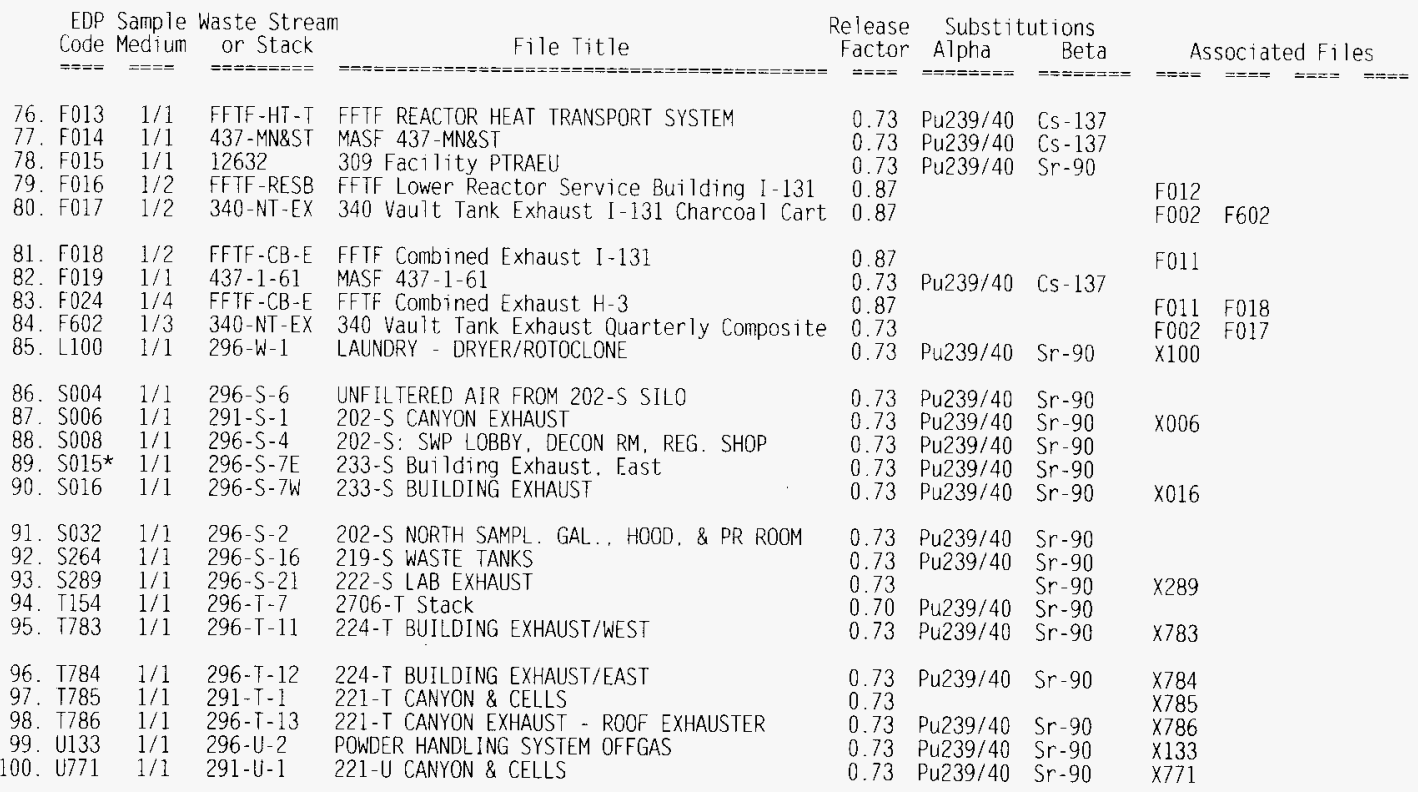


AUDIT

$$
05 / 13 / 96
$$

EDP Sample waste Stream Code Medium or Stack

101. U777 1/1 296-U-4

102. 4878

103. $\$ 003$

104 w010

105. W011

1/1

$1 / 1$ $296-\mathrm{U}-13$ $296-W-3$ $696-W-1$

$696-w^{\prime}-2$

106. $\$ 096$

107. W111

108. W117

109 . W145* $1 / 1$

110. $W 190 \quad 1 / 1$
296-5- 18 $296-5-15$ $296-\mathrm{T}-17$ $296-5-25$ $296-P-23$

$296-P-22$ 296-P-28 $296-5-22$ $296-\mathrm{T}-18$ 296-U-11

291-S-1 $296-\mathrm{A}-40$ $296-\mathrm{S}-7 \mathrm{~W}$ $296-\mathrm{P}-26$ 296-A-12

296-A- 17 $296-P-16$ $296-C-5$ $296-\mathrm{A}-25$ $296-C-6$
File Title

U03 CALCINATORS \& CONCENTRATORS

224-U LOADOUT ROOM EXHAUST

213-W WASTE COMPACTOR

WSCF LABORATORY MAIN STACK EXHAUST

WSCF. NSL COUNTING ROOM EXHAUSTER

242-S BUILDING EXHAUST

EXHAUSTER FOR ALL SX TANKS

242-T CELLS

EXHAUSTER FOR ALL SY TANKS

EXHAUSTER FOR ALL SY TANKS

ALL SY ANNULI

BACKUP EXHAUSTER FOR ALL SY TANKS

244-S SALTWELL RECEIVER \& ANNUL.

244-TX SALT WELL RECEIVER \& ANN

296-U-11 STACK SAMPLER

202-S CANYON EXHAUST

241-AP TANK FARM EXHAUST

233-S BUILDING EXHAUST

241-AY, AZ EXHAUSTER, BACKUP RP 296-A-17

296-A-12 STACK SAMPLER

Release Substitutions

Factor Alpha Beta

Associated Files

$\begin{array}{llll}0.80 & \text { Pu239/40 } & & \times 777 \\ 0.73 & \text { Pu239/40 } & \text { Sr-90 } & \text { X878 } \\ 0.73 & \text { Pu239/40 } & \text { Sr-90 } & \\ 0.73 & \text { Pu239/40 } & \text { Sr-90 } & \\ 0.73 & \text { Pu239/40 } & \text { Pu239/40 } & \\ 0.69 & \text { Pu239/40 } & \text { Sr-90 } & \text { X096 } \\ 0.84 & & & \times 111 \\ 0.81 & \text { Pu239/40 } & \text { Sr-90 } & \\ 0.72 & & & \times 145 \\ 0.79 & & & \times 190\end{array}$

0. $80 \quad$ Pu239/40 Sr-90

0.73

0.72

0.70

0.73

0.73

0.80

0.73

0.70

0.73

41-AY AZ TANKS

105-C \& 106-C TANK EXHAUSTER

244-CR VAULT CELL \& VESSEL VENT

244-A LIFT STATION CATCH TANK

296-C-6 STACK SAMPLER

\subsection{1 \\ 0.83 \\ 0.81 \\ 0.73}

-

S016

E039

E058

E059 F026

E068

E069

E080

E083 E084 


$$
05 / 13 / 96
$$

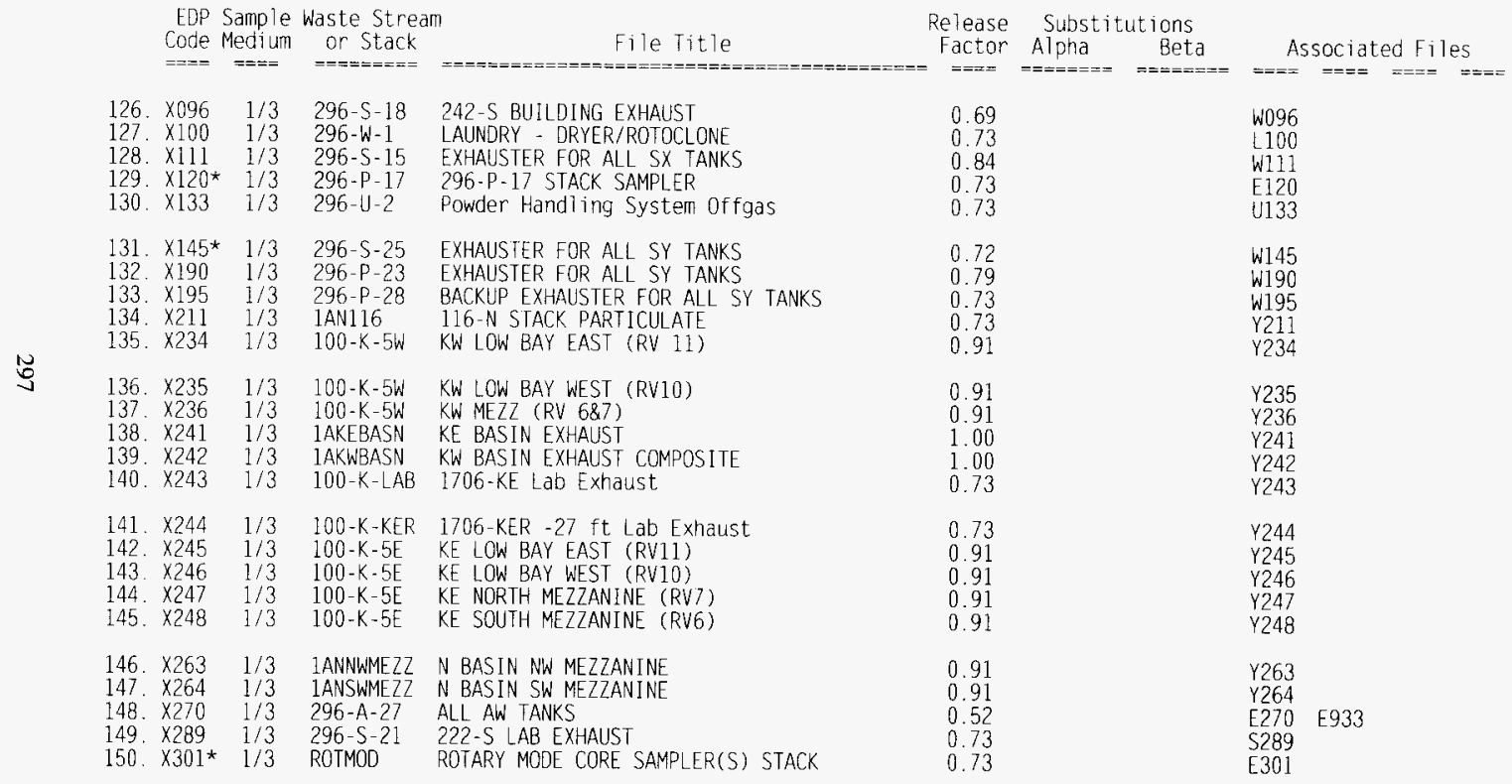



$05 / 13 / 96$

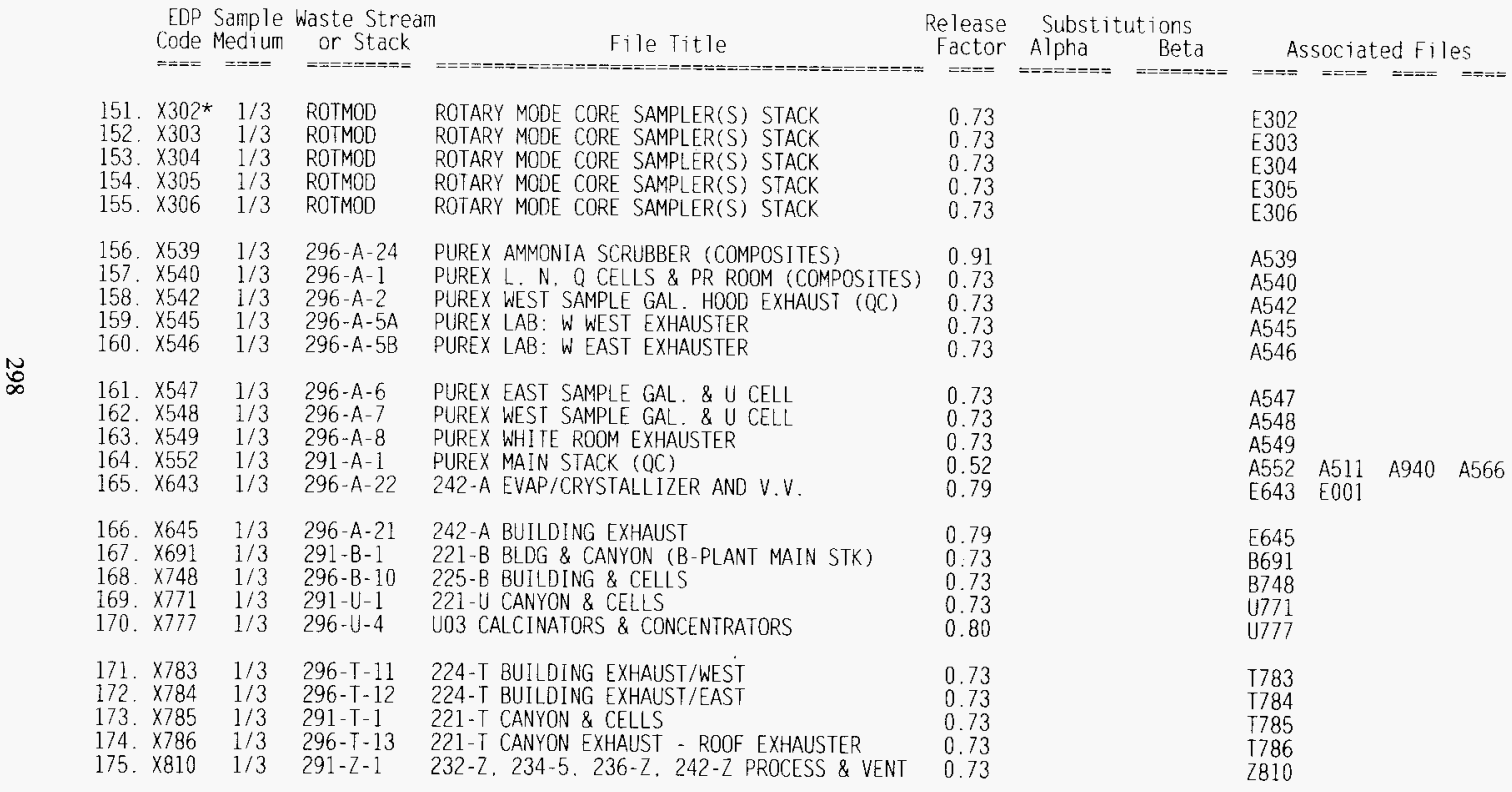




$$
05 / 13 / 96
$$

\begin{tabular}{|c|c|c|c|c|c|c|c|c|}
\hline & $\begin{array}{l}E \text { EP } \\
\text { Code } \\
====\end{array}$ & $\begin{array}{l}\text { Sample } \\
\text { Medium } \\
====\end{array}$ & $\begin{array}{l}\text { Waste Stream } \\
\text { or Stack } \\
========\end{array}$ & 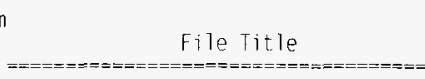 & $\begin{array}{l}\text { Release } \\
\text { Factor } \\
====-\end{array}$ & $\begin{array}{l}\text { Substitı } \\
\text { Alpha } \\
=====--\end{array}$ & $\begin{array}{l}\text { utions } \\
\text { Beta } \\
==-=-=\end{array}$ & $\begin{array}{l}\text { Associated Files } \\
==-=====0\end{array}$ \\
\hline $\begin{array}{l}176 . \\
177 . \\
178 . \\
179 \\
180 .\end{array}$ & $\begin{array}{l}\times 813 \\
\times 878 \\
\times 880 \\
\times 882 \\
\times 884\end{array}$ & $\begin{array}{l}1 / 3 \\
1 / 3 \\
1 / 3 \\
1 / 3 \\
1 / 3\end{array}$ & $\begin{array}{l}296-Z-3 \\
296-1 U-13 \\
296-S-22 \\
296-T-18 \\
296-11-11\end{array}$ & $\begin{array}{l}241-\text { I SIJMP \& VESSEL VENTS } \\
\text { LOADOUT ROOM EXHAUST } \\
244-S \text { SAL TWELL RECIEVER \& ANNULUS } \\
244-\text { TX SALT WEIL RECEIVER \& ANNULUS } \\
296-U \text { - } 11 \text { STACK SAMPLER }\end{array}$ & $\begin{array}{l}0.73 \\
0.73 \\
0.72 \\
0.70 \\
0.73\end{array}$ & & & $\begin{array}{l}2813 \\
\text { U878 } \\
\text { w880 } \\
\text { W882 } \\
\text { W884 }\end{array}$ \\
\hline $\begin{array}{l}181 . \\
182 . \\
183 . \\
184 . \\
185 .\end{array}$ & $\begin{array}{l}\times 886 \\
\times 901 \\
\times 913 \\
Y 211 \\
Y 212\end{array}$ & $\begin{array}{l}1 / 3 \\
1 / 3 \\
1 / 3 \\
1 / 1 \\
1 / 1\end{array}$ & $\begin{array}{l}296-B-28 \\
296-A-29 \\
296-Z-5 \\
\text { IAN116 } \\
\text { IAN116 }\end{array}$ & $\begin{array}{l}296-B-28 \text { STACK SAMPLER } \\
\text { ALL AN TANKS } \\
2736-2 B \text { BUILDING EXHAUST } \\
116-N \text { SIACK PARTICULATE } \\
116-N \text { STACK CHARCOAL }\end{array}$ & $\begin{array}{l}0.72 \\
0.53 \\
0.73 \\
0.73 \\
0.73\end{array}$ & & & $\begin{array}{l}E 886 \\
E 901 \\
2913 \\
\times 211\end{array}$ \\
\hline $\begin{array}{l}186 . \\
187 \\
188 . \\
189 \\
190\end{array}$ & $\begin{array}{l}Y 213 \\
\text { Y214 } \\
\text { Y } 215 \\
\text { Y22 } \\
\text { Y } 222\end{array}$ & $\begin{array}{l}1 / 1 \\
1 / 1 \\
1 / 1 \\
1 / 1 \\
1 / 1\end{array}$ & $\begin{array}{l}\text { 1AN116 } \\
\text { EAL } \\
\text { EAL } \\
\text { IAN09Z1 } \\
\text { 1AN0971 }\end{array}$ & $\begin{array}{l}\text { 116-N STACK (NOBLE GASES) } \\
\text { EAL H0-64-4276 } \\
\text { EAL H0-64-4230 } \\
109 \text {-20NE I PART ICILATE } \\
109 \text { ZONE I CHARCOAL }\end{array}$ & $\begin{array}{l}0.73 \\
0.73 \\
0.73 \\
0.73 \\
0.73\end{array}$ & $\begin{array}{l}\text { Pur39/40 } \\
\text { Pu239/40 }\end{array}$ & $\begin{array}{l}5 r-90 \\
5 r-90\end{array}$ & \\
\hline $\begin{array}{l}191 . \\
192 . \\
193 . \\
194 . \\
195 .\end{array}$ & $\begin{array}{l}Y 223 \\
Y 231 \\
Y 232 \\
Y 233 \\
\text { Y234 }\end{array}$ & $\begin{array}{l}1 / 1 \\
1 / 1 \\
1 / 1 \\
1 / 1 \\
1 / 1\end{array}$ & $\begin{array}{l}\text { 1AN0971 } \\
\text { 1AN09C6 } \\
\text { 1AN09C6 } \\
\text { 1AN09C6 } \\
\text { 100-K-5W }\end{array}$ & $\begin{array}{l}109 \text { ZONE I (NOBLE GASES) } \\
\text { CELL \#6 PARTICULATE } \\
\text { CELL \#6 CHARCOAL } \\
\text { CELL \#6 (NOBLE GASES) } \\
\text { KW LOW BAY EAST (RV 11) }\end{array}$ & $\begin{array}{l}0.73 \\
0.73 \\
0.73 \\
0.73 \\
0.91\end{array}$ & & & $\times 234$ \\
\hline $\begin{array}{l}196 . \\
197 . \\
198 . \\
199 . \\
200 .\end{array}$ & $\begin{array}{l}Y 235 \\
Y 236 \\
Y 241 \\
Y 242 \\
Y 243\end{array}$ & $\begin{array}{l}1 / 1 \\
1 / 1 \\
1 / 1 \\
1 / 1 \\
1 / 1\end{array}$ & $\begin{array}{l}100-K-5 W \\
100-K-5 W \\
\text { IAKEBASN } \\
\text { 1AKWBASN } \\
100-K-L A B\end{array}$ & $\begin{array}{l}\text { KW LOW BAY WEST (RV 10) } \\
\text { KW MEZZ. (RV 6\&7) } \\
\text { KE BASIN EXHAUSE } \\
\text { KW BASIN EXHAUST } \\
1706-\mathrm{KE} \text { Lab EXhaust }\end{array}$ & $\begin{array}{l}0.91 \\
0.91 \\
1.00 \\
0.73 \\
0.73\end{array}$ & & & $\begin{array}{l}\times 235 \\
\times 236 \\
\times 241 \\
\times 242 \\
\times 243\end{array}$ \\
\hline
\end{tabular}




$$
\text { 05/13/96 }
$$

\begin{tabular}{|c|c|c|c|c|c|c|c|c|}
\hline & $\begin{array}{l}\text { EDP S } \\
\text { Code M } \\
====\end{array}$ & $\begin{array}{l}\text { Sample } \\
\text { Medi uim } \\
====\end{array}$ & $\begin{array}{l}\text { Waste Stream } \\
\text { or Stack } \\
======= \pm\end{array}$ & $\begin{array}{l}\text { File Title } \\
==-1\end{array}$ & $\begin{array}{l}\text { Release } \\
\text { Factor } \\
====\end{array}$ & $\begin{array}{l}\text { Substit } \\
\text { Alpha } \\
=======\end{array}$ & $\begin{array}{l}\text { ions } \\
\text { Beta }\end{array}$ & $\begin{array}{l}\text { Associated Files } \\
-=====-======\end{array}$ \\
\hline $\begin{array}{l}201 . \\
202 . \\
203 \\
204 .\end{array}$ & $\begin{array}{l}Y 244 \\
\text { Y } 245 \\
\text { Y } 246 \\
\text { Y } 247 \\
\text { Y } 248\end{array}$ & $\begin{array}{l}1 / 1 \\
1 / 1 \\
1 / 1 \\
1 / 1 \\
1 / 1\end{array}$ & $\begin{array}{l}100-K-K E R \\
100-K-5 E \\
100-K-5 E \\
100-K-5 E \\
100-K-5 E\end{array}$ & $\begin{array}{l}\text { 1706-KER - } 27 \mathrm{ft} \text { Lab Exhaust } \\
\text { KE LOW BAY EAST (RV11) } \\
\text { KE LOW BAY WEST (RV10) } \\
\text { KE NORTH MEZZANINE (RV7) } \\
\text { KE South Mezzanine (RV } 6 \text { ) }\end{array}$ & $\begin{array}{l}0.73 \\
0.91 \\
0.91 \\
0.91 \\
0.91\end{array}$ & & & $\begin{array}{l}\times 244 \\
\times 245 \\
\times 247 \\
\times 248\end{array}$ \\
\hline $\begin{array}{l}206 . \\
207 \\
208 \\
209 \\
210\end{array}$ & $\begin{array}{l}Y 251 \\
Y 252 \\
r 253 \\
Y 254 \\
r 255\end{array}$ & $\begin{array}{l}1 / 1 \\
1 / 1 \\
1 / 1 \\
1 / 1 \\
1 / 1\end{array}$ & $\begin{array}{l}\text { 1AN09Z2 } \\
\text { 1AN09Z2 } \\
\text { 1AN09Z3 } \\
\text { 1AN0975 } \\
\text { 1AN09Z4 }\end{array}$ & $\begin{array}{l}\text { ZONE } 1 \text { I (EF 7.8) PARTICULATE } \\
\text { ZONE II (EF 7,8) CHARCOAL } \\
\text { ZONE II (EF 10) PARTICULATE } \\
\text { ZONE. II (EF 10) CHARCOAL } \\
\text { ZONE IV (EF 14.15) PARTICULATE }\end{array}$ & $\begin{array}{l}0.73 \\
0.73 \\
0.73 \\
0.73 \\
0.73\end{array}$ & & & \\
\hline $\begin{array}{l}211 . \\
212 \\
213 \\
214 \\
215\end{array}$ & $\begin{array}{l}Y 256 \\
Y 257 \\
Y 258 \\
Y 259 \\
Y 260\end{array}$ & $\begin{array}{l}1 / 1 \\
1 / 1 \\
1 / 1 \\
1 / 1 \\
1 / 1\end{array}$ & $\begin{array}{l}\text { 1AN0974 } \\
\text { IAN05DOCN } \\
\text { IAN05DOCN } \\
\text { IANO5DCN } \\
\text { IANO5DCN }\end{array}$ & $\begin{array}{l}\text { ZONE IV (EF } 14,15 \text { ) CHARCOAL } \\
\text { DUMMY SPACER EXHAUST PART ICULATE } \\
\text { DLMMYY SPACER EXHAUSF CHARCOAL } \\
14^{\circ} \text { DECON FACILITY PAR ICULATE } \\
14^{\circ} \text { DECON FACILITY CHARCOAL }\end{array}$ & $\begin{array}{l}0.73 \\
0.73 \\
0.73 \\
0.73 \\
0.73\end{array}$ & & & \\
\hline $\begin{array}{l}216 . \\
217 . \\
218 . \\
219 . \\
220 .\end{array}$ & $\begin{array}{l}Y 261 \\
Y 262 \\
Y 263 \\
Y 264^{*} \\
Y 265\end{array}$ & $\begin{array}{l}1 / 1 \\
1 / 1 \\
1 / 1 \\
1 / 1 \\
1 / 1\end{array}$ & $\begin{array}{l}\text { IAN05TRN } \\
\text { IAN05TRN } \\
\text { IANNWMEZZZ } \\
\text { 1ANSWMEZZ } \\
\text { IAN07ROOF }\end{array}$ & $\begin{array}{l}\text { TRANSFER AREA PART ICULATE } \\
\text { TRANSFER AREA CHARCOAL } \\
\text { N BASIN NW MEZZANINE } \\
\text { N BASIN SW MEZZANINE } \\
\text { 107-N EXHAUST PARTICULATE }\end{array}$ & $\begin{array}{l}0.91 \\
0.73 \\
0.91 \\
0.91 \\
0.73\end{array}$ & & & $\begin{array}{l}\times 263 \\
\times 264\end{array}$ \\
\hline $\begin{array}{l}221 . \\
222 . \\
223 . \\
224 . \\
225 .\end{array}$ & $\begin{array}{l}Y 266 \\
2802 \\
7810 \\
2813 \\
Z 814\end{array}$ & $\begin{array}{l}1 / 1 \\
1 / 1 \\
1 / 1 \\
1 / 1 \\
1 / 1\end{array}$ & $\begin{array}{l}1 \text { ANOTROOF } \\
296-Z-6 \\
291-z-1 \\
296-2-3 \\
296-2-14\end{array}$ & $\begin{array}{l}\text { 107-N EXHAUST CHARCOAL } \\
2736-2 \text { A BUILDING EXHAUST } \\
232-2,234-5.236-2.242-2 \text { PROCESS \& VENT } \\
241-2 \text { SUMP \& VESSEL VENTS } \\
232-2 \text { BUILDING EXHAUST }\end{array}$ & $\begin{array}{l}0.73 \\
0.73 \\
0.73 \\
0.73 \\
0.73\end{array}$ & $\begin{array}{l}\text { Pu239/40 } \\
\text { Pu239/40 }\end{array}$ & $\begin{array}{l}S r-90 \\
S r-90 \\
S r-90 \\
S r-90\end{array}$ & $\begin{array}{l}\times 810 \\
\times 813\end{array}$ \\
\hline
\end{tabular}


AUDI I $05 / 13 / 96$

FUP Sample waste Strearn
Code Medium or Stack
$=======$ File Title

226. $2913 \quad 1 / 1 \quad 296-2-5 \quad 2736-2 B$ BIJILDING EXHAUST $\quad 0.73$ Pu239/40 $5 r-90$ 227. $7915 \quad 1 / 1$ 296-2-15 243-Z LOW LEVEL WASTE WATER TREATMENT FAC1 0.73 Pu239/40 $\mathrm{Sr}-90$

* Denotes empty file.

Total 227 files, of which 20 are empty. 
WHC-SD-EN-TI-308 Rev.0

This page intentionally left blank. 
WHC-SD-EN-TI-308 Rev.0

5.2 Audit Report for Liquid Effluent Files 


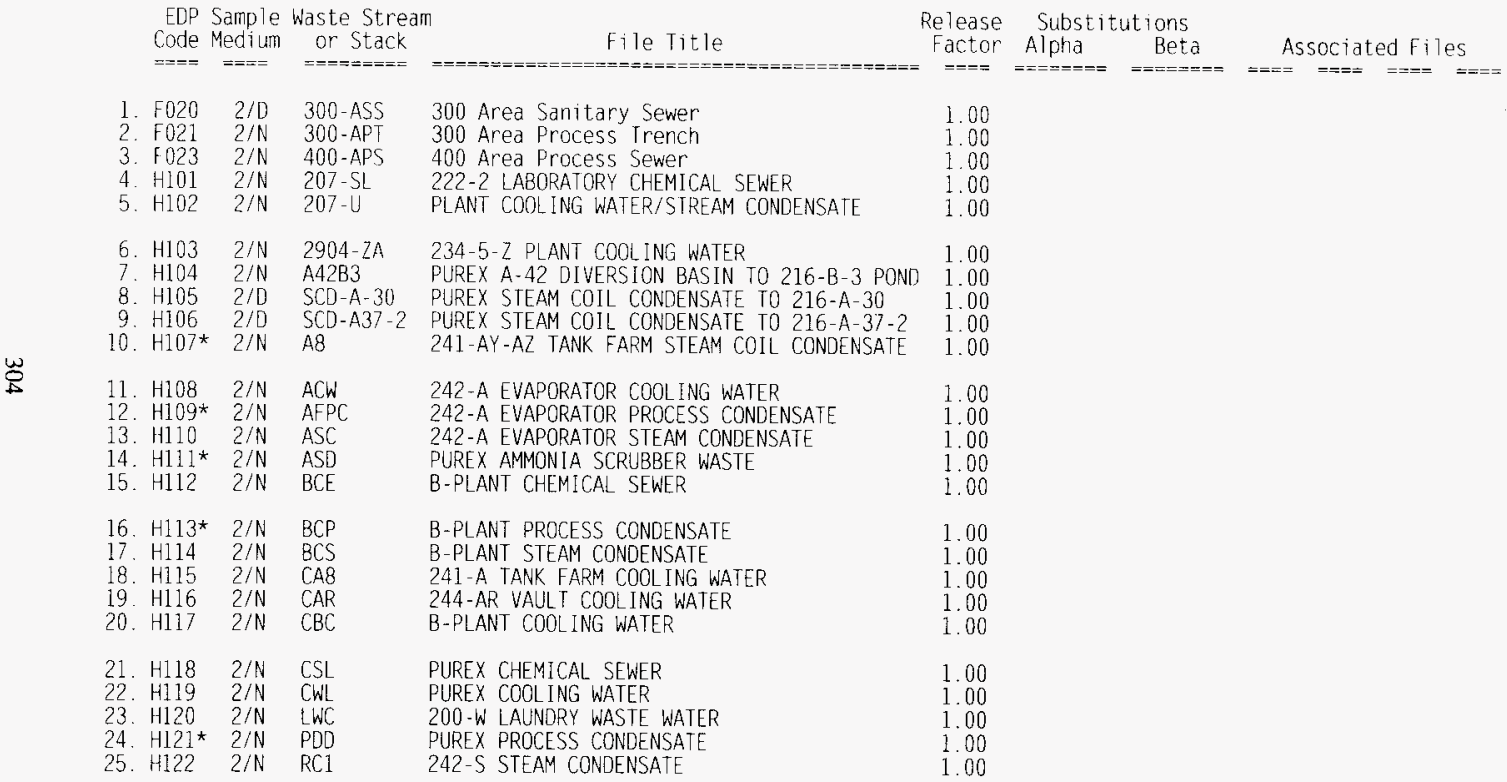




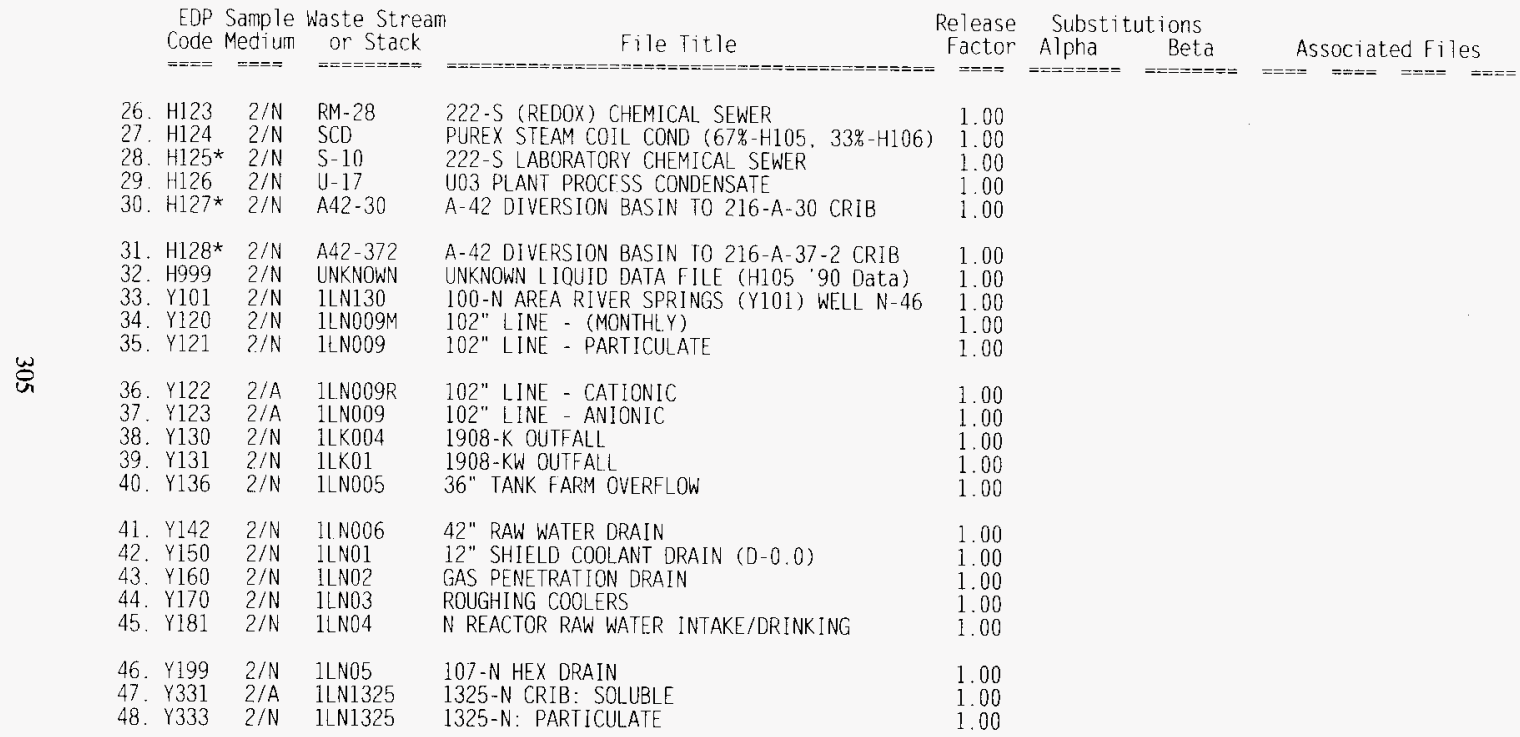

* Denotes empty file.

Total 48 files, of which 8 are empty. 
WHC-SD-EN-TI-308 Rev.0

This page intentionally left blank. 


\section{WHC-SD-EN-TI-308 Rev.0}

\section{0 $\quad{ }^{241} \mathrm{Pu}$ CALCULATIONS}

Concentrations of ${ }^{241} \mathrm{Pu}$ are calculated using weight percent data provided from $\mathrm{PFP}$ along with the ${ }^{239 / 240} \mathrm{Pu}$ and ${ }^{238} \mathrm{Pu}$ concentrations from the applicable emission point. The ${ }^{241} \mathrm{Pu}$ releases are calculated using the procedure in WHC-IP-1066. The concentrations calculated using the ${ }^{238} \mathrm{Pu}$ data were used to calculate the CY-1995 releases, since they were typically larger than values calculated using ${ }^{239 / 240} \mathrm{Pu}$ concentrations 


\section{Weight Percent Values Used for CY-1995 Releases}

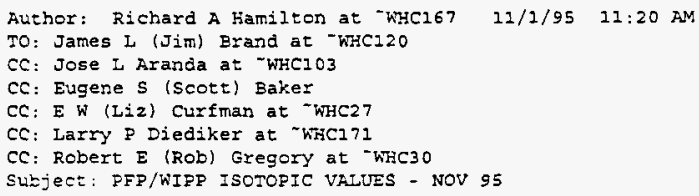

\begin{tabular}{|c|c|c|c|}
\hline Pu - ZミB & 0.0288 & $n t z$ & of \\
\hline$F \div-\Sigma \equiv 9$ & 93.8251 & & $"$ \\
\hline$P u-\div 60$ & $5.71 \div 6$ & & $"$ \\
\hline$P y-\geq 41$ & 0.4149 & & $n$ \\
\hline$P L-=42$ & 0.0196 & & $"$ \\
\hline$\therefore m-241$ & 0.2616 & & $"$ \\
\hline
\end{tabular}




\section{SUPPLEMENTAL DATA FOR CALCULATING Pu-241 CONCENTRATIONS}

\section{Isotopic Compostion of PFP Plutonium:}

\begin{tabular}{|l|r|r|}
\hline & (Weight \%) & (fraction) \\
\hline Pu-239 & 93.8251 & 0.938251 \\
Pu-240 & 5.7116 & 0.057116 \\
Pu-241 & 0.4149 & 0.004149 \\
Pu-238 & 0.0288 & 0.000288 \\
Pu-242 & 0.0196 & 0.000196 \\
\hline & & \\
\hline Total & 100.0000 & 1.000000 \\
\hline
\end{tabular}

Specific Acitivities of Plutonium Isotopes:

\begin{tabular}{|l|r|c|c|}
\hline & $\begin{array}{c}\text { Half } \\
\text { Life } \\
(y)\end{array}$ & $\begin{array}{c}\text { Half } \\
\text { Life } \\
(\mathrm{s})\end{array}$ & $\begin{array}{c}\text { Specific } \\
\text { Activity } \\
(\text { Ci/g) }\end{array}$ \\
\hline $\mathrm{Pu}-239$ & 24131 & $7.610 \mathrm{E}+11$ & $6.197 \mathrm{E}-02$ \\
$\mathrm{Pu}-240$ & 6569 & $2.072 \mathrm{E}+11$ & $2.267 \mathrm{E}-01$ \\
$\mathrm{Pu}-241$ & 14.4 & $4.541 \mathrm{E}+08$ & $1.030 \mathrm{E}+02$ \\
$\mathrm{Pu}-238$ & 87.75 & $2.767 \mathrm{E}+09$ & $1.711 \mathrm{E}+01$ \\
$\mathrm{Pu}-242$ & 375800 & $1.185 \mathrm{E}+13$ & $3.930 \mathrm{E}-03$ \\
\hline
\end{tabular}

Specific Activity of Pu-239 and Pu-240 Mixture:

For $1 \mathrm{~g}$ of mixture: $\quad 0.942618 \mathrm{~g} \mathrm{Pu}-239$

$$
0.057382 \mathrm{~g} \mathrm{Pu}-240
$$

Isotopic activities in mixture:

$$
\begin{aligned}
(0.942618 \mathrm{~g})(0.06197 \mathrm{Ci} / \mathrm{g}) & =5.8410 \mathrm{E}-02 \mathrm{Ci} \mathrm{Pu}-239 \\
(0.057382 \mathrm{~g})(0.2267 \mathrm{Ci} / \mathrm{g}) & =1.3007 \mathrm{E}-02 \mathrm{Ci} \mathrm{Pu}-240
\end{aligned}
$$

Activity per gram of mixture (SpA):

$$
\mathrm{SpA}=(\mathrm{Ci} \mathrm{Pu}-239)+(\mathrm{Ci} \text { Pu240 })=7.1418 \mathrm{E}-02 \mathrm{Ci} / \mathrm{g} \text { of } \mathrm{Pu}-239 / 240 \text { mixture }
$$


WHC-SD-EN-TI-308 Rev.0

\section{Calculating PUReX Pu-241 CONCentrations}

291-A-1

\begin{tabular}{|c|c|c|c|c|c|c|c|c|c|c|}
\hline $\begin{array}{c}\text { Composite } \\
\text { Date }\end{array}$ & $\begin{array}{c}\text { Pu-239/240 } \\
\text { Concentration } \\
\text { (uCi/mL) }\end{array}$ & $\begin{array}{r}\text { Sample } \\
\text { Volume } \\
\text { (cuft) }\end{array}$ & $(\mathrm{mL})$ & $\begin{array}{c}\text { Pu-239/240 } \\
\text { Activity } \\
\text { (uCi) }\end{array}$ & $\begin{array}{c}\text { Pu-239/240 } \\
\text { Mass } \\
\text { (g) }\end{array}$ & $\begin{array}{c}\text { Pu-239 } \\
\text { Mass } \\
\text { (g) }\end{array}$ & $\begin{array}{c}\text { Total } \\
\text { Pu } \\
\text { Mass } \\
\text { (a) }\end{array}$ & $\begin{array}{c}P u-241 \\
\text { Mass } \\
\text { (g) }\end{array}$ & $\begin{array}{l}\text { Pu-24t } \\
\text { Activity } \\
\text { (uCi) }\end{array}$ & $\begin{array}{c}\text { Pu-241 } \\
\text { Concentration } \\
\text { (uCl/mL) }\end{array}$ \\
\hline $12 / 21 / 94-03 / 29 / 95$ & $2.7 E-15$ & $280 \mathrm{E}+0.5$ & $7.93 \mathrm{E}+09$ & $214 \mathrm{E}-05$ & $300 \mathrm{E}-10$ & $2.83 \mathrm{E}-10$ & $301 \mathrm{E}-10$ & 1.25E-12 & 1.29E-04 & $1.62 E-14$ \\
\hline $03 / 29 / 95.06 / 21 / 95$ & $1.5 \mathrm{E} \cdot 15$ & $240 E+05$ & $6.80 E+09$ & $1.02 \mathrm{E}-05$ & $1,43 \mathrm{E}-1 \mathrm{D}$ & $1.35 E-10$ & $1.43 \mathrm{E} \cdot 10$ & $595 E-13$ & 6. 13E-05 & 9.02E-15 \\
\hline $06 / 21 / 95-09 / 27 / 95$ & $9.7 E-16$ & $2.80 E+05$ & $793 E+09$ & $769 E-06$ & $1.08 E-10$ & 1.02E-10 & $1.08 \mathrm{E}-10$ & $449 E-13$ & $462 \mathrm{E} \cdot 05$ & 5.83E-15 \\
\hline $09 / 27 / 95-12 / 20 / 95$ & $9.2 \mathrm{E}-16$ & $2.20 E+05$ & 6. $23 E+09$ & $5.73 E-06$ & $8.03 E-11$ & $7.57 \mathrm{E}-11$ & $8.06 \mathrm{E}-\$ 1$ & $3.35 \mathrm{E}-13$ & $3.45 E-05$ & 5.53E-15 \\
\hline
\end{tabular}

\begin{tabular}{|c|c|c|c|c|c|c|c|c|c|}
\hline $\begin{array}{l}\text { Composite } \\
\text { Date }\end{array}$ & $\begin{array}{c}\text { Pu-238 } \\
\text { Concentratıon } \\
\text { (uCi/mL })\end{array}$ & $\begin{array}{l}\text { Sample } \\
\text { Volume } \\
\text { (cuft) }\end{array}$ & $(\mathrm{mL})$ & $\begin{array}{c}\text { Pu-238 } \\
\text { Activity } \\
\text { (uGi) }\end{array}$ & $\begin{array}{c}\text { Pu-238 } \\
\text { Mass } \\
\text { (g) }\end{array}$ & $\begin{array}{l}\text { Total } \\
\text { Pu } \\
\text { Mass } \\
\text { (a) } \\
\end{array}$ & $\begin{array}{c}\text { Pu-241 } \\
\text { Mass } \\
\text { (d) }\end{array}$ & $\begin{array}{l}\text { Pu-241 } \\
\text { Activity } \\
\text { (LCi) }\end{array}$ & $\begin{array}{c}\text { Pu-241 } \\
\text { Concentration } \\
\text { (uCi/mL) }\end{array}$ \\
\hline $03 / 29 / 95-06 / 21 / 95$ & $1.2 E-16$ & $2.40 E+05$ & $6.80 \mathrm{E}+09$ & $8.16 E-07$ & $4.77 E-14$ & 1. $65 E-10$ & $6.87 E-13$ & $707 E-05$ & $1.04 E-14$ \\
\hline $09 / 27 / 95-12 / 20 / 95$ & $6.2 \mathrm{E}-17$ & $2.20 \mathrm{E}+05$ & $623 \mathrm{E}+09$ & $3.86 \mathrm{E}-07$ & $2.26 \mathrm{E}-14$ & $7.84 \mathrm{E} \cdot \uparrow 1$ & $3.25 E-13$ & $335 E-05$ & $5.38 E-15$ \\
\hline
\end{tabular}

296-A-1

\begin{tabular}{|c|c|c|c|c|c|c|c|c|c|c|}
\hline $\begin{array}{l}\text { Composite } \\
\text { Date }\end{array}$ & $\begin{array}{c}\text { Pu-239/240 } \\
\text { Concentration } \\
\text { (uCi/mL) }\end{array}$ & $\begin{array}{l}\text { Sample } \\
\text { Volume } \\
\text { (cuft) }\end{array}$ & $(\mathrm{mL})$ & $\begin{array}{c}\text { Pu- } 239 / 240 \\
\text { Activity } \\
\text { (UCi) }\end{array}$ & $\begin{array}{c}\text { Pu-239/240 } \\
\text { Mass } \\
\text { (g) }\end{array}$ & $\begin{array}{c}\mathrm{Pu}-239 \\
\text { Mass } \\
\text { (c) }\end{array}$ & $\begin{array}{c}\text { Total } \\
\text { Pu } \\
\text { Mass } \\
\text { (g) }\end{array}$ & $\begin{array}{c}\text { Pu-241 } \\
\text { Mass } \\
\text { (O) }\end{array}$ & $\begin{array}{l}\text { Pu-241 } \\
\text { Activity } \\
\text { (uCi) }\end{array}$ & $\begin{array}{c}\text { Pu-241 } \\
\text { Concentration } \\
\text { (uCijmL) }\end{array}$ \\
\hline $12 / 21 / 94-03 / 29 / 95$ & 4. $7 \mathrm{E}-14$ & $170 E+05$ & $4.81 E+09$ & $2.26 \mathrm{E}-04$ & $3.17 \mathrm{E}-09$ & $299 \mathrm{E}-09$ & $318 \mathrm{E}-09$ & 1.32E-11 & 1.36E-03 & $2.82 E-13$ \\
\hline $03 / 29 / 95-06 / 21 / 95$ & $1.4 \mathrm{E}-14$ & $1.60 \mathrm{E}+05$ & $4.53 E+09$ & $634 \mathrm{E}-05$ & $8.38 E-10$ & 8 $37 E-10$ & $892 E \cdot 10$ & $370 \mathrm{E}-12$ & 3. $81 \mathrm{E}-04$ & B.41E-14 \\
\hline $06 / 21 / 95-09 / 27 / 95$ & $1.2 \mathrm{E}-14$ & $1.90 \mathrm{E}+05$ & $5.38 E+09$ & $646 \mathrm{E}-05$ & $9.04 \mathrm{E}-10$ & $852 \mathrm{E}-10$ & $908 \mathrm{E}-10$ & $3.77 \mathrm{E}-12$ & 388E-04 & 7.21E-14 \\
\hline $09 / 27 / 95-12 / 20 / 95$ & $1.9 E \cdot 14$ & $1.60 \mathrm{E}+05$ & $4.53 E+C 9$ & 8.5†E-05 & 1. 21E-09 & 1.14E-09 & $121 E-09$ & $5.02 \mathrm{E} \cdot 12$ & $5.17 E-04$ & $1.14 E-13$ \\
\hline
\end{tabular}

\begin{tabular}{|c|c|c|c|c|c|c|c|c|c|}
\hline $\begin{array}{l}\text { Composite } \\
\text { Date }\end{array}$ & $\begin{array}{c}\text { Pu-238 } \\
\text { Concentratıon } \\
\text { (uCi/mL) }\end{array}$ & $\begin{array}{l}\text { Sample } \\
\text { Volume } \\
\text { (cuft) }\end{array}$ & $(\mathrm{mL})$ & $\begin{array}{c}\text { Pu-238 } \\
\text { Activity } \\
\text { (uCi) }\end{array}$ & $\begin{array}{c}\text { Pu-238 } \\
\text { Mass } \\
\text { (g) }\end{array}$ & $\begin{array}{c}\text { Total } \\
\text { Pu } \\
\text { Mass } \\
\text { (g) }\end{array}$ & $\begin{array}{l}\text { Pu-241 } \\
\text { Mass } \\
(g)\end{array}$ & $\begin{array}{l}\text { Pu-241 } \\
\text { Activity } \\
\text { (uCi) }\end{array}$ & $\begin{array}{c}\text { Pu-241 } \\
\text { Concentration } \\
\text { (uCi/mL) }\end{array}$ \\
\hline $12 / 21 / 94-03 / 29 / 95$ & $3.4 E \cdot 15$ & $170 E+05$ & $4.81 E+09$ & $164 \mathrm{E}-05$ & 9.57E-13 & 3.32E-09 & $1.38 \mathrm{E}-11$ & $1.42 \mathrm{E}-\mathrm{O} 3$ & 2.95E-13 \\
\hline $03 / 29 / 95-06 / 21 / 95$ & 6. $3 E-16$ & $160 E+05$ & $453 E+09$ & $285 E-06$ & $167 \mathrm{E}-13$ & $579 E-10$ & $240 E-12$ & $247 E-04$ & $5,46 E-14$ \\
\hline $06 / 21 / 95-09 / 27 / 95$ & $64 E-16$ & $190 E+05$ & $5.38 E+09$ & $344 \mathrm{E}-06$ & $201 E-13$ & $6.99 E-10$ & $290 \mathrm{E}-12$ & 2.99E-04 & $5.55 E-14$ \\
\hline $09 / 27 / 95-12 / 20 / 95$ & $9.6 \mathrm{E}-16$ & $1.60 E+05$ & $453 E+09$ & $435 E-06$ & $254 \mathrm{E}-13$ & $8.83 \mathrm{E}-10$ & $366 \mathrm{E}-12$ & $377 \mathrm{E}-04$ & $8.32 E-14$ \\
\hline
\end{tabular}


WHC-SD-EN-TI-308 Rev.0

CALCULATING PFP Pu-241 CONCENTRATIONS

291-Z-1

\begin{tabular}{|c|c|c|c|c|c|c|c|c|c|c|}
\hline $\begin{array}{l}\text { Composite } \\
\text { Date }\end{array}$ & $\begin{array}{c}\begin{array}{c}\text { Pu-239/240 } \\
\text { Concentration } \\
(\mathrm{uC} / \mathrm{mL})\end{array} \\
\end{array}$ & $\begin{array}{l}\text { Sample } \\
\text { Volume } \\
\text { (cuft) }\end{array}$ & $(m L)$ & $\begin{array}{c}\text { Pu-239/240 } \\
\text { Activity } \\
\text { (UCi) }\end{array}$ & $\begin{array}{c}\mathrm{Pu}-239 / 240 \\
\text { Mass } \\
(\mathrm{g}) \\
\end{array}$ & $\begin{array}{c}P u-239 \\
\text { Mass } \\
(g)\end{array}$ & $\begin{array}{c}\text { Total } \\
\text { Pu } \\
\text { Mass } \\
\text { (g) }\end{array}$ & $\begin{array}{c}\text { Pu-241 } \\
\text { Mass } \\
\langle g\rangle .\end{array}$ & $\begin{array}{l}\text { Pu-241 } \\
\text { Activity } \\
\text { (uCi) }\end{array}$ & $\begin{array}{c}\text { Pu-241 } \\
\text { Concentration } \\
\text { (uCifmL) }\end{array}$ \\
\hline $12 / 21 / 94-03 / 29 / 95$ & 2.7E-15 & $2.80 E+05$ & $793 E+09$ & $214 \mathrm{E}-05$ & $300 \mathrm{E}-10$ & $283 E-10$ & 3.01E-10 & $125 \mathrm{E}-12$ & 1.29E-04 & $1.62 E-14$ \\
\hline $03 / 29 / 95-06 / 21 / 95$ & 1.5E-15 & $240 E+05$ & $680 E+09$ & $102 E-05$ & $143 \mathrm{E}-10$ & $135 \mathrm{E}-10$ & 1.43E-10 & $595 \mathrm{E}-13$ & 6 13E-05 & 9.02E-15 \\
\hline $05 / 21 / 95-09 / 27 / 95$ & $97 E-16$ & $2.80 \mathrm{~F}+05$ & $7.93 E+09$ & $769 \mathrm{E}-06$ & $1.08 E-10$ & $102 \mathrm{E}-10$ & $1.08 \mathrm{E}-10$ & $4.49 E-13$ & 4.62E-05 & $5.83 E-15$ \\
\hline $09 / 27 / 95-12 / 20 / 95$ & $9.2 E-16$ & $2.20 E+0.5$ & $6.23 E+C 9$ & $5.73 \mathrm{E} .06$ & B $03 E-11$ & $757 \mathrm{E}-11$ & $8.06 \mathrm{E}-11$ & $3.35 \mathrm{E}-13$ & $345 E-05$ & 5.53E-15 \\
\hline
\end{tabular}

\begin{tabular}{|c|c|c|c|c|c|c|c|c|c|}
\hline $\begin{array}{l}\text { Composite } \\
\text { Date }\end{array}$ & $\begin{array}{c}\mathrm{Pu}-238 \\
\text { Concentration } \\
(\mathrm{uCi} / \mathrm{mL}) \\
\end{array}$ & $\begin{array}{l}\text { Sample } \\
\text { Volume } \\
\text { (cuft) }\end{array}$ & $(\mathrm{mL})$ & $\begin{array}{l}\text { Pu-238 } \\
\text { Activity } \\
\text { (UCi) }\end{array}$ & $\begin{array}{c}\text { Pu-238 } \\
\text { Mass } \\
(g)\end{array}$ & $\begin{array}{c}\text { Total } \\
\text { Pu } \\
\text { Mass } \\
\text { (g) }\end{array}$ & $\begin{array}{c}\mathrm{Pu}-241 \\
\text { Mass } \\
(q)\end{array}$ & $\begin{array}{l}\text { Pu-241 } \\
\text { Activity } \\
\text { (uCi) }\end{array}$ & $\begin{array}{c}\text { Pu-241 } \\
\text { Concentration } \\
\text { (uCilmL) }\end{array}$ \\
\hline $12 / 21 / 94-03 / 29 / 95$ & $79 \mathrm{E}-16$ & $2.80 \mathrm{E}+05$ & $7.93 E+09$ & $6.26 \mathrm{E}-06$ & $3.66 \mathrm{E}-13$ & $1.27 \mathrm{E}-09$ & $5.27 \mathrm{E}-12$ & $5.43 E-04$ & $6.85 E-14$ \\
\hline $03 / 29 / 95-06 / 21 / 95$ & $2.8 \mathrm{E}-16$ & $2.40 E+05$ & $6.80 E+09$ & 1.90E-06 & 1.14E-13. & $3.86 \mathrm{E}-10$ & $1.60 \mathrm{E}-12$ & 1.65E-0.4 & $2.43 E-14$ \\
\hline $06 / 21 / 95-09 / 27 / 95$ & $32 \mathrm{E}-16$ & $280 E+05$ & $7.93 E+09$ & $2.54 \mathrm{E}-06$ & $148 \mathrm{E}-13$ & $5.15 E-10$ & $2.14 E-12$ & $2.20 \mathrm{E}-04$ & $2.77 E-14$ \\
\hline $09 / 27 / 95-12 / 20 / 95$ & $4 \mathrm{OE}-16$ & $2.20 E+05$ & $623 E+09$ & $2.49 \mathrm{E}-06$ & $1.46 \mathrm{E}-13$ & $5.06 \mathrm{E}-10$ & 2.10E-12 & $2.16 \mathrm{E}-04$ & $3.47 \mathrm{E}-44$ \\
\hline
\end{tabular}

296-Z-3

\begin{tabular}{|c|c|c|c|c|c|c|c|c|c|c|}
\hline $\begin{array}{l}\text { Composite } \\
\text { Date }\end{array}$ & $\begin{array}{c}\text { Pit-239/240 } \\
\text { Concentration } \\
\text { (uGi/mL) }\end{array}$ & $\begin{array}{l}\text { Sample } \\
\text { Volume } \\
\text { (cuft) }\end{array}$ & $(\mathrm{mL})$ & $\begin{array}{c}\text { Pu-239/240 } \\
\text { Activity } \\
\text { (uCi) }\end{array}$ & $\begin{array}{c}\text { Pu-239/240 } \\
\text { Mass } \\
\text { (g) } \\
\end{array}$ & $\begin{array}{l}\text { Pu-239 } \\
\text { Mass } \\
\text { (a) }\end{array}$ & $\begin{array}{c}\text { Total } \\
\text { Pu } \\
\text { Mass } \\
\text { (g) }\end{array}$ & $\begin{array}{c}P_{u-241} \\
\text { Mass } \\
\text { (g) }\end{array}$ & $\begin{array}{c}\text { Pu-241 } \\
\text { Activity } \\
\text { (uCi) }\end{array}$ & $\begin{array}{c}\text { Pu-241 } \\
\text { Concentration } \\
\text { (uClimL) }\end{array}$ \\
\hline $12 / 21 / 94-03 / 29 / 95$ & $4.8 \mathrm{E}-16$ & 2.90E+05 & $595 E+09$ & $2.85 E-06$ & 4. OOE-11 & $3.77 E-1 \uparrow$ & $4.02 E-11$ & $167 \mathrm{E}-13$ & $\uparrow .72 E-05$ & $2.88 E \cdot 15$ \\
\hline $03 / 29 / 95-06 / 21 / 95$ & $20 \mathrm{E}-15$ & $1.50 \mathrm{E}+05$ & $425 E+09$ & 8.50E-06 & 1. 19E-10 & 1. $12 \mathrm{E}-10$ & $1.20 \mathrm{E}-10$ & 4.96E-13 & $5.11 E-05$ & $1.20 E-14$ \\
\hline $06 / 21 / 95 \cdot 09 / 27 / 95$ & 3.3E-15 & $1.90 E+05$ & $5.38 E+09$ & 1.78E-05 & $2.49 \mathrm{E}-10$ & $234 \mathrm{E}-10$ & $2.50 \mathrm{E}-10$ & $104 E-12$ & $1.07 E-04$ & $1.98 \mathrm{E} \cdot 14$ \\
\hline $09 / 27 / 95-12 / 20 / 95$ & $9.7 \mathrm{E}-16$ & $190 \mathrm{E}+05$ & $5.38 E+09$ & $522 E-06$ & $7.31 \mathrm{E}-11$ & 6.89E-11 & 7.34E-11 & 3.05E-13 & 3.14F-05 & 5.A3E-15 \\
\hline
\end{tabular}

\begin{tabular}{|c|c|c|c|c|c|c|c|c|c|}
\hline $\begin{array}{l}\text { Composite } \\
\text { Date }\end{array}$ & $\begin{array}{c}\text { Pu-23日 } \\
\text { Concentration } \\
\text { (uCimL })\end{array}$ & $\begin{array}{l}\text { Sample } \\
\text { Volume } \\
\text { (cuft) }\end{array}$ & $(\mathrm{mL})$ & $\begin{array}{l}\text { Pu-238 } \\
\text { Activity } \\
\text { (uCi) }\end{array}$ & $\begin{array}{c}\text { Pu-238 } \\
\text { Mass } \\
(g)\end{array}$ & $\begin{array}{c}\text { Total } \\
\text { Pu } \\
\text { Mass } \\
\text { (g) }\end{array}$ & $\begin{array}{c}P \cup-241 \\
\text { Mass } \\
\text { (g) }\end{array}$ & $\begin{array}{l}\text { Pu-241 } \\
\text { Activity } \\
\text { (uCi) }\end{array}$ & $\begin{array}{c}\text { Pu-241 } \\
\text { Concentration } \\
\text { (uCi/mL) }\end{array}$ \\
\hline $12 / 21 / 94-03 / 29 / 95$ & $6.4 \mathrm{E}-16$ & $210 E+05$ & $5.95 E+09$ & $3.81 E-06$ & $2.22 E-13$ & $7.72 E \cdot 10$ & $3.20 \mathrm{E}-12$ & $3.30 E-04$ & $5.55 E-14$ \\
\hline $03 / 29 / 95-06 / 21 / 95$ & $2.2 \mathrm{E}-15$ & $1.50 E+05$ & $4.25 \mathrm{E}+09$ & $9.35 \mathrm{E}-06$ & 5. $46 E-13$ & $1.90 E-09$ & $7.87 \mathrm{~F}-12$ & 8. $10 \mathrm{E}-04$ & 1.91E-13 \\
\hline $05 / 21 / 95-09 / 27 / 95$ & 4.1E-15. & $190 \mathrm{E}+05$ & $5.38 \mathrm{E}+09$ & $2.2 \mathrm{tE}-05$ & 1. $29 E-12$ & 4. $\triangle 8 E-09$ & $1.86 \mathrm{E}-11$ & $1.91 E-03$ & $3.55 E-13$ \\
\hline $09 / 27 / 95-12 / 20 / 95$ & $72 \mathrm{E}-18$ & $1.90 E+05$ & $5.38 \mathrm{E}+09$ & $3.87 \mathrm{E}-06$ & $226 E-13$ & 7 96E-10 & $326 \mathrm{E}-12$ & $336 \mathrm{E}-04$ & $6.24 E-14$ \\
\hline
\end{tabular}

